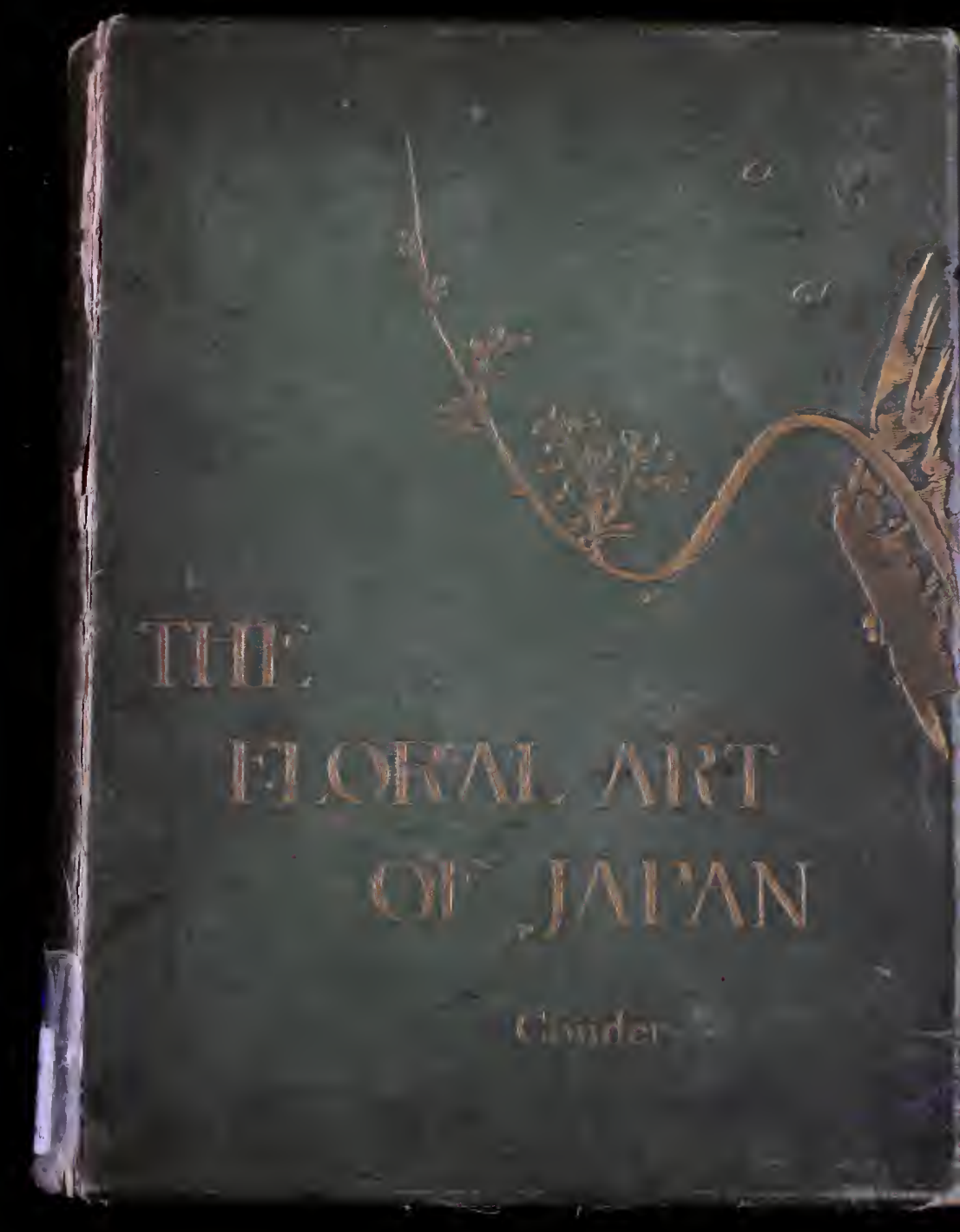


$\checkmark$

.

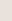



THE

FLORAL ART

$\mathrm{OF}$

JAPAN. 
TIIE

\section{FLORAL ART OF JAPAN:}

BEING

A SECOND AND REVISED EDITION

OF

\section{THE FLOWERS OF JAPAN}

AND THE

ART OF ILORAL ARRANGENENT.

LY

JOSIAH CONDER, F.R.I.B.A.,

WITH ILLLSTRATIONS BY JAPANESE ARTISTS.

\section{[ALL. HHHIh BESHKLD.]}

TOK10 :

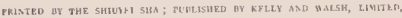

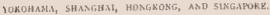


$19 / 9 / 22$
mins Petture - Se

41

20 9 


\section{I'REFACE.}

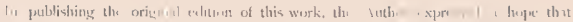

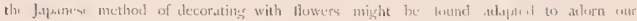

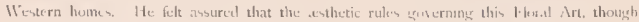

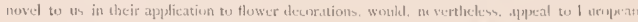

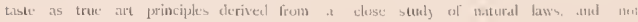

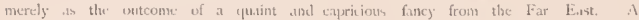

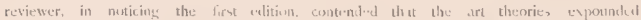

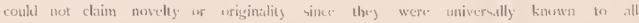

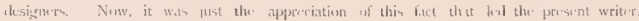

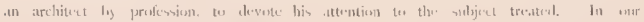

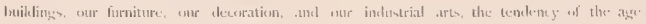
is to discard false: and meaniogless designs. and ter follesw the orue primeiples hide stown las

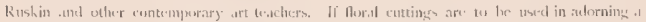

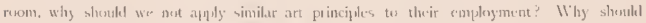

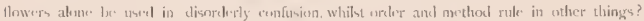
Working with stome, lrick. timber. metal. paint. or plaster. we aim at expressing

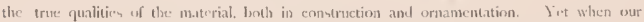
material is the freshest am lovelies that the earts jields we are comtent to thic it in a

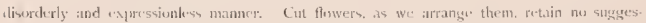

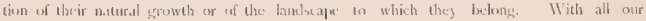

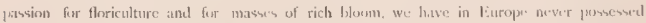

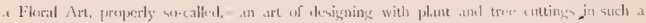

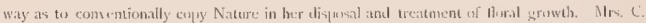

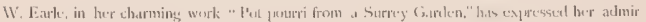
ing appeciation of the Japanest mote: of arranging liswers for embellishing roums, and fus given several exanjples showing how such a methoul moty he applied to Einglish flowers.

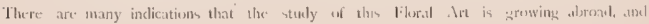


I'

that it will ontinu- to unflue ise ur ane of cuttimes for chamber decoratios. The writer cloes not supplose that the styte of Ilower arangements followed by the ]apsinse will "ver be alopted complete, with all the quaint traditions and subtle ethics that surround it in the land of its origin, but an explanation of these details is desirable in order to show the: rationate which evolvel the Art in the liands of this cultured people, and to indicate the lines upon which a suitable Luropean adajtation may be Lasecl.

The above considerations encournge the Author in issuing a secund and revised edition of his original work. Aeklitional wutline plates and figures in the text have been introduced, as well as new coloured prints from clesigns expressly made by Ogata Getkio. one of the best living artists of the l'optlar sehool. The teat has been augnented to some extent, partly in the body of the work, and partly in an appenclix. The somewhat lenethy titce of the first edition has been clispened with, ant the abbreviated on: "Floral Art of Japan" adoptecl.

JOSIAH CONDER.

Tokio, May, Is99. 


\section{CONTENTS.}

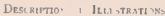

THE. FLOWERS OF JAPAN.

INTKODLITH:

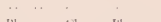

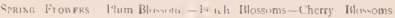

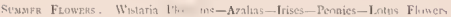

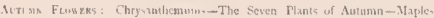

\section{TIL ARRANGEMIFI OF HLOWEIS}

Istronitin . .

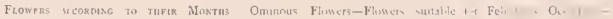

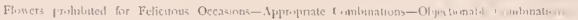

Histoh, sar Tileos

Lisf in Distrinution

Gelection of Miteriat

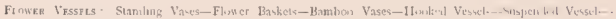

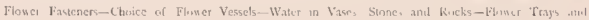

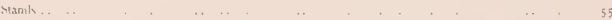

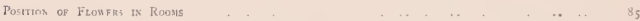

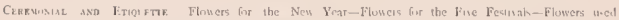

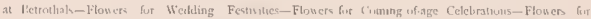

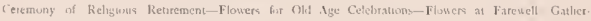
mos-Flowers for the Sick-Flowers hefore Hounchold Shanes-Flmers at Death AnnuersinesFlowers at Prayers for Ran or Fine Wealber-Flusers uved at Mhou Viemug-Flowen fur IJunse

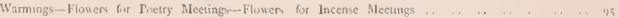

Nastptsatios Tieatnuent of Cuttings beforc Arranging-Treatment of Stein Ends to prodnce Succulency-

Nethods of bending Sicms and Eranches-Preservann of Ileavy Blossoms-Pantung of Lcaves and

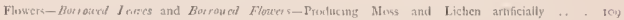

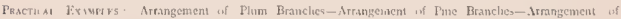

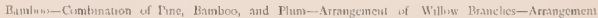

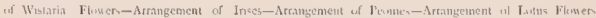

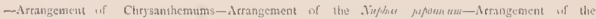

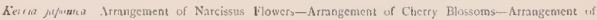

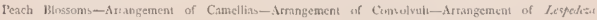

Floners-Arrangencut of Rhouht Mpoweti-Arangement of Leaf Orchul-Arrangement af Maple

Branches-Miscellancists

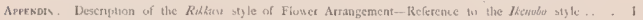




\title{
IOESCRIPTION OF ILLUSTR.ATIONS.
}

\author{
COLOURED PL.ISES.
}

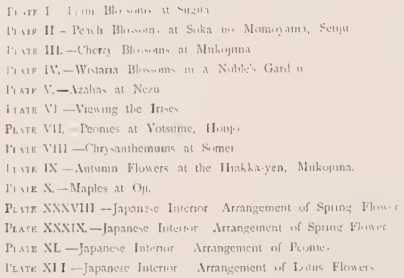

\section{OUTLINE ILATES.}

1 1 sIE XI - Thatgrani un Riklira lane.

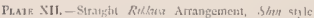

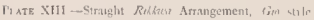
PIATE XIV.- Straght Rihkice Arrangement, Sir wile

PI.nTe XV. - Bent Rekiow Arrangement, Shat styte.

PLATE XYJ.-Bent Rikkan Arrangement, Giv styje. PLitr XVII - Bent Rukhers Arrangemeht, Se style.

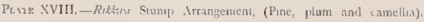

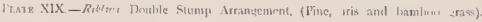




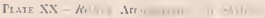

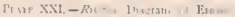

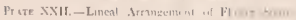

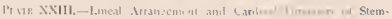

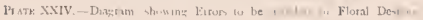

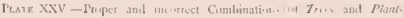

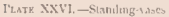

Platr XXlit. - Fowcehasm, Tubs, and Bashel-

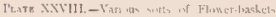

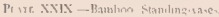

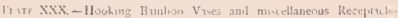

PLAm XXNI - Pillartaldets mit Flower standi

PLita XXXII. - Suvpendesl Vesseds of Bronze, Wood, anil Bamlua

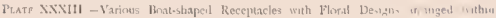

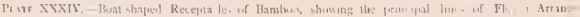
meats therein

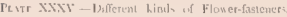

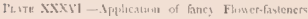

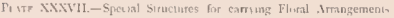

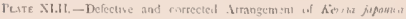

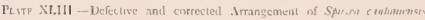

PLate XLIV.-Defective and correctel Arrangement of Leaf Orehil

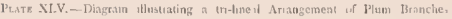

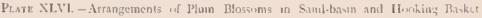

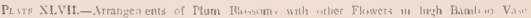

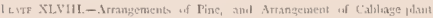

1'LATE. XLIX-Arrangements of Bamliun

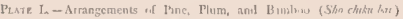

Peitr: L.I.-Amangenents of Whlow Branches

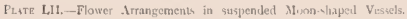

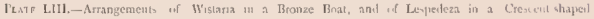
Vessil.

PLATE LIV-Micthol of arringmp hises.

PLite LY:-Cimpletecl Arrangements of Iriscs in Standing-virede.

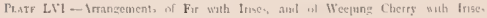

Ptate I.VIl. - Pairod Arraggement of Inaes, anil Asters.

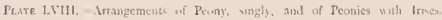

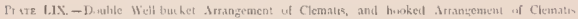

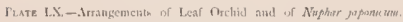

PLire LXI - Atrangements of Convolvali and of Duonthus supertus

Pt.ste I.XII.-Arrangement of Clirysanthemums.

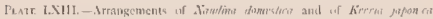

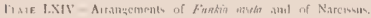




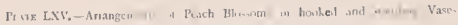

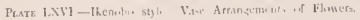

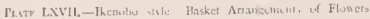

PLATE L.XVIII,- Thethotio bf le Hasin Arrangements of Floners

PLixe I.XIX-1henotus style Basin Arrangements of Flomers

\section{ILLIISTRATIONS PRINTEN WIIH THE TEXI}

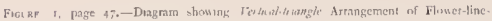

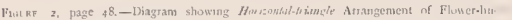

Jiar $\mathrm{KE}$ 3. page 55,-Long-neckeil Staniling-tase conlaimug Arangement of Fine

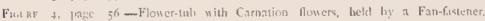

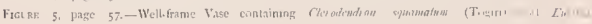
axmotr (Sisuki).

Figixe, 6 , page $3 \hat{\delta},-$ Hokny Flowut-linsket contuming Cowwolvul.

Figine 7. page 5\%,-Sise, Flowerbashet contasnug Chysanthemums.

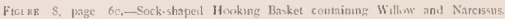

Figt kF 9. jage hi-Bambin Vaxe wh Chrysuntiemums.

Proter 10, page 65. - flooking Vase in emlirouderel bag, confamung Clirysanticmums,

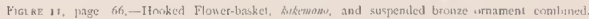

Figt נe 12, page 67.-1 pair of suspended boat shajed Vases uith Elower-armungemento.

Funve 13. jage 65.-Fluwer-satt holdung small Basket conthinng Cherry Blosioms.

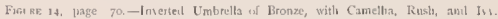

Protue r5, page 71.-Floner-chanot with Autuma Plants and Grasses.

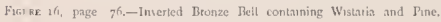

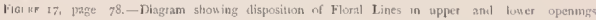
of high Bambon Vases.

Fugl

Fteinz 19, page $\mathrm{S}_{3}$ - Table for Incense-burner with Shelf for Flowers below.

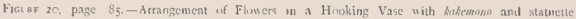

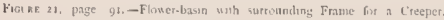

Pricker 22, page yj-Flumer-ball

Ficter, 23, 1 age grio-Utensils and Tools for artanging Flowers.

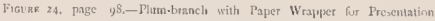

Figure 25, page 100.-Arrangement of Irises in fromt of a picture

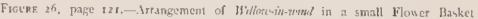

Figlese 27, page 12z-Arrangement of Wolana, in large Basin wh Stontz;

FrougF $2 S$, page tz8.-Arrangement of Sutnmer Chrysanthersums.

Figere zy. page izR-Method of using three small Chryanthernun sprigs with other grewth. 


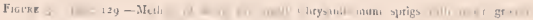

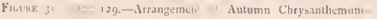

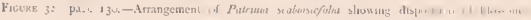

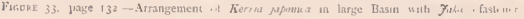

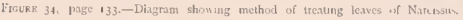

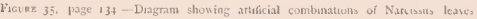

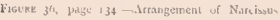

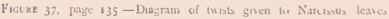

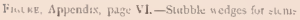

Fuxkr, Ain endex, page VII - Frame wedges fur stubs 



\title{
THE FLOWERS OF JAPAN.
}

\author{
INTRODUCTION.
}

\author{
"Fluwer seem ustended for the sulue if ondinaty humanss

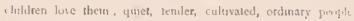

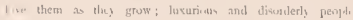 \\ inits is them gatheret." - Joum Riskis,
}

\begin{abstract}
SE: NE of the general impressions which exist abroad with regarel to Japan, is that of a lanel (ii) unrivaled in the abundance and beaty of its flowers, -a belinf that nature has lavished

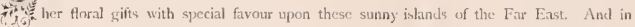

T a great measure is this popular fancy justified, considering the imposing display of flowering trces and shrubs to be seen near the principal cities at certain seasons. In the sense. bowever, of profusion in wild floral plants, it nust bs admitted that certain Western countries possess atractions which Japanese scenery can scarcely boast. The traveller, whose rural wanderings nearer home have made him familiar with furze and heather-clad moors, flowersprinkled meadows, or hills and forests girdled and carpeted with blossoning plants, will miss in Japan some of these charming adornments of natural lindscape. There is one short season in the year.-when the rice is young and green, and the birom of the honcy-sec-nted rape phant spreads broal stretches of jellow on the plains,- that recalls tu the mind the soft fowering verdure of other lands: but the rice culture, with its enclless itrigated tertaces, and the lsigh bamboo-grass, which covers every uncultivated hill and gien, deprives the scenery of all but a bricf and passing suggestion of the coluring of Western meadows and uphand. Tiger lilies, fratillaries, latuebells, and numerous other wild flowers yrow on the Ilains and downs, huried in the high coarse bamboograss. The comparative scarcity of groups of wild flowering jlants, as a colour feature to the landscapes, is, however, to some cxtent mikte up for by the blossoming trees, displaying in turn soft and vivid masses of colour imid the verdant foliage of the hill-sides. The wild cancllin, azilia, magnolia, plun, peach, and cherry are the most important of these flowering trees, the varical flaming tints of the azalia contributing chiefly to the brightness of the seenery. The blossom of the wild cluerry tres, which alsounds in
\end{abstract}


Yezn, adds to the wen if i landicale of this Northern island an efiect as of soft clouds e, in pale. prearly tint, which the juets liken to mine upen the mountains,

The fower charm as it exists in Japan is not, as tsewhere, purcly one of rural or pastoral associtions. It is closcly and inscparably connected with the national custons and the national art. The artistic character of the Japanese people is most strikingly clisplayed in their methols of interpreting the simpler of natural beautics. That extravagant taste which demands for its satisfaction the grand, rarc, or novel, and is unmoved by the modest attractions of more familiar surroundinzss, is little shared by the masses of this people, who bestow their chicf sympathies on the humbler aspects of nature. Though the more majestic scencry of the country has served as a constant thene for the painters and poets of the Cutre and nobility, the fopular art has always been an expression of the chily life of the people, and of those simple, natural surroundings, customs, and faniliar beliefs which contribute to its enjoyment. The recurring months of the year, and the various duties, []casures, and poctic fancics which custom has associated with them, form the inexhaustible source from which artists of all kinds have looked for inspiration. The common flowers of the scasons have been given a prominent place in the fete-day calendar. Almost cvory month is known by its special blossoms, and the important citics have groves and gardens devoted to their public display;. The festivals of the numerous Shinto deities are celebrated monthly in the difierent towns by strect hiss at which the choicest specimens of flowering trees and plants are exposed for salc. The secret. then, of Japan's flonal fame ant floral enchantment lies rather in the care that her people bestow upon Nature's simpler gifts than in any transcentent wealch of procluction. Treasured chicfly as heralds of the seasons, and as insepanble from the favourite pursuits and pastimes of out-door life, Japanese fowers are by no moans estcemed in proportion to their scarcity or difficulty of culcure. The isolated merit of mrity, so mucly sought after in the IVest, has here little or no attraction. The native forists are not deficient in Horicultural skill. and produce in certain blossoms forms of considerable artificial beauty, but the popular taste shows a partiality for the more ordinary and familiar flowers, endeared by custom and tradition.

Flonter aidwing excursions, toggether with such pastimes as Shell grathering. Mnshroom picking, and Alon viecring, form the favourite occupations of the holiday secker throughout the year. By a pretty fancy, even the snow-clad landscape is regarded as IV'inter's floral display, and Snow virwing is included as one of the flower festivals of the year. The Chinese calendar, used formerly by the Japanesc, fitted in admirably with the jortical sticcession of flowers. Horn, the Japanese Spriner, opened with the New Yenr, which commenced about February; and was heralded by the appearance of the Plum blossoms. 


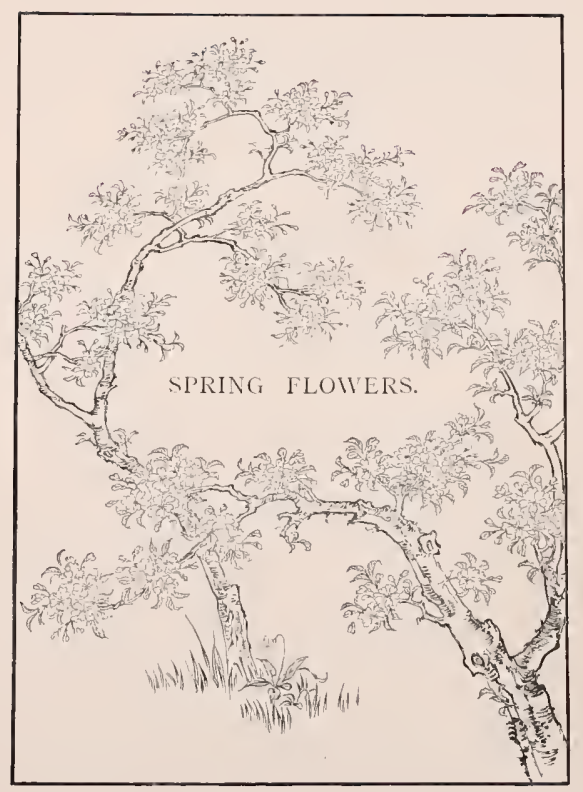





\title{
SPRING FLOTIERS.
}

\author{
PIUM BLUSTUMLS.
}

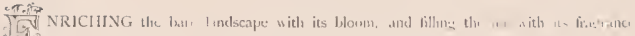

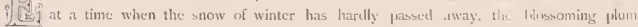
for tree has come to lxe regarded with especial fondness ly the Jayram si: Combineal F" with the evergreen pine and bamboo, it forms a floral triad, ealled the Sho-dhiku bar, supposed to be expressive of enduring happiness, and uscd as a decorative symbol on many felicitous occasions. The plum blossom being the earlicst to bloom in the frear, is often referred to as "the chlest brother of the bundred fowers." Quick in scizing the peculiar features which distinguish one growth from another, to the extent almost of a tendency to caricature them, the Japanese have been chicfly attracted by the rugged and angular character of the plum tret, its stiff, straight sloots, and sparsc, studeled arrangensent of buds and blossons. Thus, a fancy has arisen for the oldest trees, which with their gnarled trunks and tender shoots exhilit these: characteristics to perfection. In them is shown the striking contrast of bent, crabbecl ange, and fresh, sigorous youth. As if to rencler this ideal more complete, it is held that the plum tree should be secm in bud rather than in full blosson.
\end{abstract}

The gardeners of the country, so dever in the training of miniature trees, find in the plum a choice olject for their skill, imitating on a small scale this fuvourite character of hudling youth grafted on to twisted and contorted ayse. Tlese tiny treces, trained in a varicty of shapes, - bent, curvel, and even spital,-with their vertical or shooping graltings of elifferent coloured blossom-sprajs, Fresh. fragrant, ind long lasting, form one of the most charming of rom decorations during the tirst months of the year.

Pocts and artists love to compare thin flowering tree with its later risal, the cherry: With the latter, they say; the blossom absorbs all interest: whereas, in the cane of the forner, atuntion is clrawn more to the shape of trusk and banclues: the cherry blossom, it is not denied, is the pretior and gayer of the two; but the plum blosson is 
min chat-1-and molest in appearance, possessing besides, its sweet odour. Enthusiastic Wranes of the cherry Hossom, however, yo so fur as to funcy they detect a delicious odour (1) this secntiess finwer. The fragrance of the plum blossom is cunstantly referred to in the hort stanas with which the poetry of the country abounds. The following free translation may be given as an cxample of one of sucli verses:-

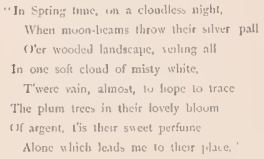

The custom of planting plum trees in groves and avenues to form pleasure resorts during hossom time, seems to be of comparatively molern date; and some of the most fumous plum groves werc originally orchards, planted for the sake of the fruit. It is said that in China. from whence Japar borrowed many of her customs and cults, this tree was lirst estecuned for its fruit alone, and in later and more atsthetic times it becane honoured for its pure hlossom and swect scent. In the carliest Japanese annals we read of a single phan tree being regularly planted is front of the. South javilion of the Palace at Naris, and of its being replaced by a cluerry tree in later limes, when the latter had in its turn become the fatourite of the Court. In connection with this Imperial custom, a pretty story

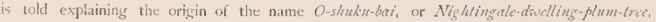
applied, even to the present day, to a favourite species of delicions odour having pink touhle hilomsom. Sone time in the tenth century the Imperial plum tree withered, and, in it was necestary to replace it. search was made for a specimen worthy of so high an honsour. Such a tree was found in the garelen of the talented daughter of a famous poet. named lino Thtrayuki, and was demanded by the officials of the Court. Not diring to resist the Imperial command, but lill of arrief at parting with her favourite plum tree, the young pretess secretly attached to its trunk a strip of paper upos which she wrote the following verse :-

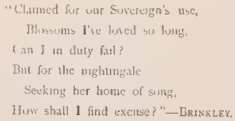




\section{PLATE I.}

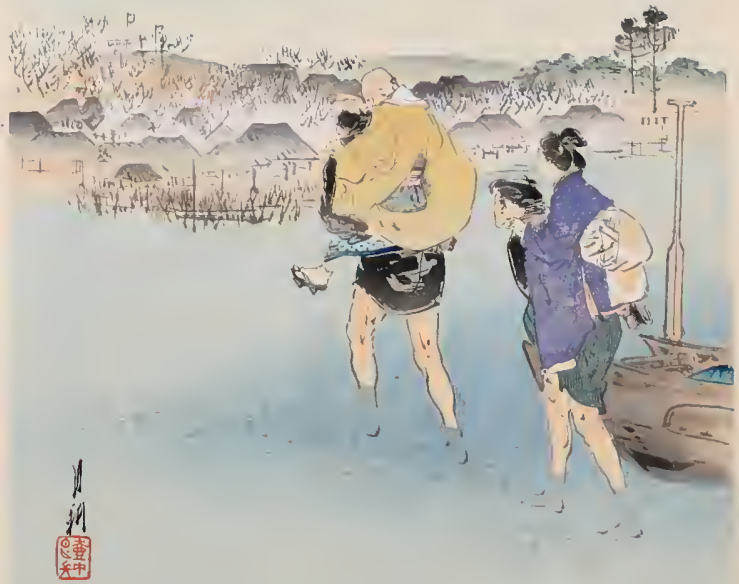




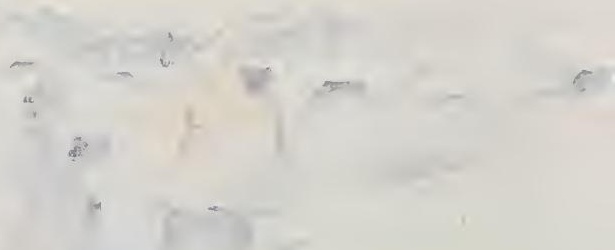


This caught the eje of the Emperor, who, toucled by the plaintiv sentiment a pressed, enquired from whose garclen the tree was taken, and ordered it to be roturned. The season of the plum blossom is mate musical with the liquicl note of the so-called Japanese niglatingale (Cottria cantans). which for this reason is inseparably associated with the plum tree in the different decorative arts. Such dual combinations, taken from the animal and vegetable kingdom, form fawourite motives for designs, Bamloo leaves with Sparrows. Pea-fowl with Pconies, Tigers with Banhoos, Storks with Pine treus, Will boar with Autumn grasses, and Deer with Maples, may be mentioned as some of these popular combinations.

In later times plum trees were planted in large numbers at rural spots near to the Imperial capitals, forming pleasure resorts for the lalies of the Court. Along the banks of the river Kizu, at a place called Tsuki-ga-se, in the jrovines of limato, fine trees of pink and white blossom extend upwards of two miles, diffusing their delicious scent around: they are what remains of quite a forest of plum trees said to have strctched for miles arouncl. The more modern truns have also their favourite plum orchards. visited by crowds of sigb-tseers at blosson cime, in February: Sugita, a village not far from Yokoliama, possesses one of the most f.inous: laving over a thousand trees, many of which are from cighty to a hundred years of agre, and which supply in the sumner mast of the fruic consuned in the Eastern Capital, Tokio. It is popularly known and firequented on account of its blossons in the carly spring, anal boasts six special varieties of tree, dis. tinguished by different fancy names having reference to the character of the flower: the principal of which are those of pink and of so-called green blossom,-for the white kind his a faint tinge of emerald.

In all, there are said to be sixty clifterent species existing in japan. The single bloswon of white or grecnish-white colour and of small sice is held most in estecm. All the white kinds are scented, but of the red some possess no perfume. There is an early plum of rect and double blossom which blooms before the Winter solstice. and is of handsone appearance, but it has little or no scent. The Japanese include several species of the Fasminum in the same category as plum trees.

Every visitor to Japan has heard of the Gixa-riv-bai, or Recumbent-dragon-plumtrees at Kameido, a famous spot in the North of Tokio. At this place there existerl, up to filty years ago, a rare and curious plum tree of great age and contorted shape. Its branches had bent. ploughing the soil, and forming new roots in fourteen places, ant it straggled over an extensive area. Owing to is writhing and suggescive slupe, it received 
the name of the Recumle at I) 5 sagon, and, yearly thad with fresh sloots and whe l, bossune of fine perfune, attracted liurge crowels of visitors. From this famous tree, fruit is said tu lave been yearly presented to the strumpun. Succumling at last to extreme ayre, it has been replaced by a number of kess imporinge ypecinens. selected on account of their more of less bent and crawling shapes. The present group of plum trees, inheriting the name. though but little of the charater of Recumbent Dragons. makes a fine show of blossoms in February, anct keeps up the popularity of the resurt.

Komurai and Kinerawa, near Kameide, ilso have blossom-groves which are much frequented.

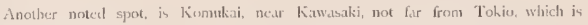
historically finous as having been uften visited by the Shogra, and possesses trees over two hundred jears of age:

At Shinjuku, another suburl, of Tokio, is a fine grove of plun trees, sonctines called the Silver-world (Gin-sckat), a $4 \mathrm{rm}$ applicel to the snow-elad landscape, and having special reference in this instance to the silver whiteness of these blossons.

The favourite: trees of single blassom are cight in number-the Hitoye-ume, Shubori. Ilesolia, Nishikin, Kotenbai, Shichare, Suzuri-shiclare, and Tokiwa-shidlare: and

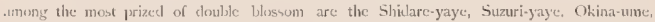
Beli-suzune. Yuncrit. and I lana-gomuri. Other trecs such as the Mangetsu. Kianoru-ume. Momodidori, Tani-no-yuki, and Miyakodori are known as the best fruit-lueariny kinds of plum tree. The illustration, Plate I, represents the plum grove: at Sugita, about five miles from lokolsama, which can be approached from the sea beach.

\section{PEXCII HLOSSONS.}

UICKLY after the plum follows the peach blosson which, though by no means sharing
the traditional esteen and admiration bestowed upon the formes, excels it in siac,
in mass, as it aypears in groves and orcharcls, contributes far more to the beauty of the 
PLATE II.

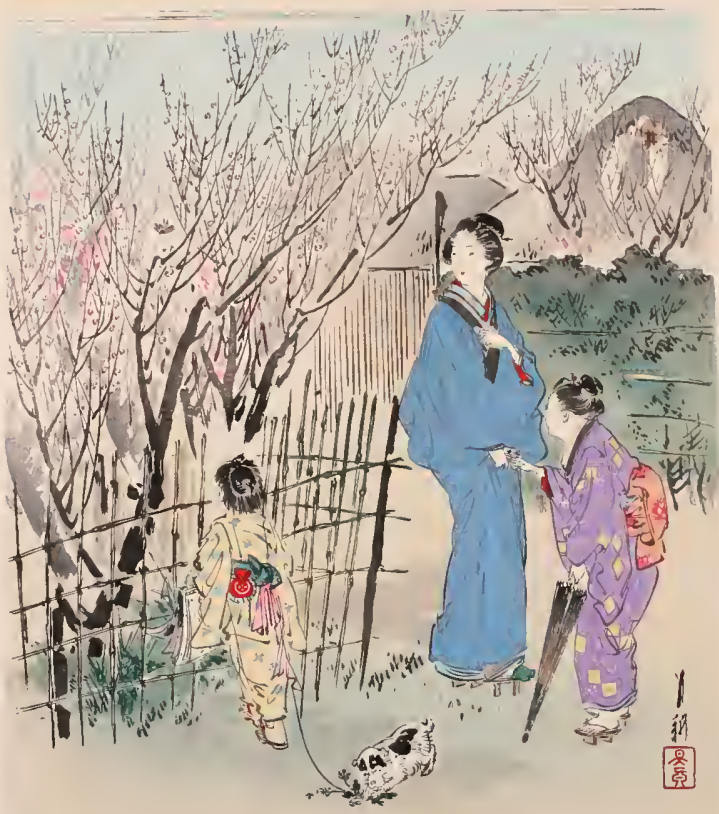



Spring landscape than its more honoured but severes brother the plum blossom, though it has not. however, hat the goost fortune to be patronized by ancient Emperors or extolled by distinguished poets. Lacking these escentials to fionl yreatness, and coming as it dhes between the plum, of classical fame ant predilection, ant the Hashy clerry of patriutic boast, it has been comparatively neglected hy the irtint and releyated to secondary rank as a decorative motive and material. The orchards of peacls trees in hossom are, howescer, much frequented by the common people who find enjoyment at any spot where blom and colour are to be seen.

Plate Il, illustrates trees in a grove called Soka-no. Monoymuna, at Sronjn, a suburb of Tokio.

\section{CIIERRY BIOSSOMS.}

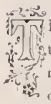

HE third month of the old Japanese Spring, corresponding with the present April. is the montly of the cherry blossom, the king of flowers in Japan. This llower is remarkable for its softness and exuberance, as contrated with the severe simplicity of the [jum blossom. The latter laooms fresh, vigorous, and leafless, in the bare anct often snow-clad landscape : the former, with its florid richness, enchanced in some cases by joung reddish leaves, is especially fitted to assert itsclf antil the greenery of butding epring-tine. But the splendour of the cherry's bloom is trancitory in comparison with the more lasting qualitics of the plum, which retains its beauty for a full month. The eleery Aowern must be viewed during the few short days of their prime, and should these days be stormy, the full glory of the sight is lost for a year. The most entlusiastic partizuns of the cherry blossom assert that it is all the more precious on account of its transient claracter. "Amons men, the simurai, anong flowers, the sakura," is a familiar saying. which well expresses the patriotic prite with which this gay flashy Hower is rogarded in Jinzan. The sentiment is also conveyeal in the following lines by one. of the national jocts :-

\footnotetext{
"shlishuma no

Sintabi gukuin $\mathrm{nu}$

Iluo tumaba

Atahis ni mincus

Yamazakara bima."
} 
"Should you ask me what is the true patriotic spirit, I answer-it is the scent of the mountain cherry tree in the morning air."

The wild cherry seems to bave existed in Japan from time immemorial, and still abounds in the wonds of the Northern island, Yezo, where the Aino aborigines apply its bark to many purposes. In ancient times, however, the plum tree, of Chinese importation. seems to have absorhed the attention of the Court and people, and it was at a later date that the cherry, the flower of the country; appears to have found its place in their affections. Though early records refer frequently to the plum, there is no mention of the cherry earlier than the time of Ricbit, als Emperor of the fitth century. This Monarch was disporting bimself with his courticrs in a pleasure boat, on a lake of the Royal park, when some petals from the wilt cherry trees of the adjoining hills fluttered into the wine cup from which he was drinking. This circumstance is said to have drawn His Majesty's notice to the beauty of this neglected blossom, and from this time arose the custon of wine drinking at the time of cherry viewing. To the present day there is a popular saying. "Without wine who can properly enjoy the sight of the cherry blossom?" It was reserved for a later Finperor, in the eighth century, to give it that importance as a national fowver which it has ever since retained. Whilst on a lunting expexition on Mount Mlikasa, in the province of Yannato, the Emperor Shomu, attracted by the beauty of the double cheriy blossoms, compased the following short verse, which be sent, with a branch of the Howers, to his favourite Consort, Komio Kogo:-

\footnotetext{
"This gathered cherry branch san starce convey

A fancy of the blessim-laden tree,

Blonmeng in sunhght; coulit I show it Thee,
}

Thinghts of tis tieauty uould done ieep away I"

Tn satisfy the curiosity of the ladies of his Cont, the Emperor ordered cherry trees to be planted near the Palace at Nara, and afterwards the custom was continued at each succeeding capital.

Yamato, the province in which were situated sevcral of these ancient capitals, is the most noted for its cherry groves, and at a spot called Yoshino a thousand trees line the path and cover the hill side. It has been a favourite fancy to compare the appearance of these trees in blosson to mists of snow upon the hills at in the verse,-." The cherry blossoms on Mount Yoslino deceive me into thinking they are snow!" 
PLATE III.

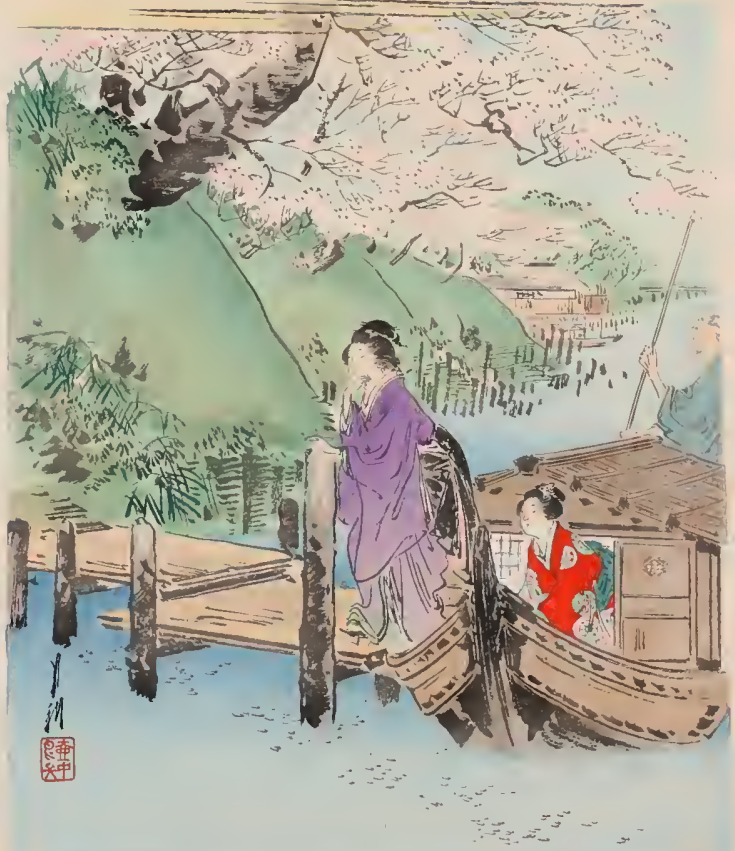



Imperial ganden parties to view the national Hower date back as early as the days of the Emperor Saga, in the ninth century. These ancient court receptions were attended by the notable literati whose amusement was to compose odes on the flowers. In the thirteenth century, the Emperor Kameyama caused a number of trees frons Yinshino to be phnted at Arashiyama, a beautiful hilly spot on the banks of the rapid river ( $)_{i}$, in the neighbourbood of kioto. Here he built a summer pavilion, and, in spring and autumn, Coust after Court visited the lovely spot, which was rendered further famous in a verse composed by one of the Imperial line - - Not second to l'oshino, is Arasliyama, where the white spray of the torrent sprinkles the cherry blossoms." This spot no lnnger possesses its Imperial pavilion, but remains a favourite resort for sight-seers from the Western capital, in the months of the cherry and the reddening maple. Numerous tea houses and booths, on the lanks of the rapicls, give a fine view of the wooded hills oppositc, amidst the Spring greenery of which may be seen the pearly white clouds of the cherry blossoms. Here the blossom-clad branches form a part of the distant lindscape, as originally beheld in their natural wildness, when they first attracted the adniration of the earlier Enperors, and b.fure their more gorgeous successors, of clouble-flower, became atranged in artificial growes and avenues.

In and near to the modern capital, Tokio, are several syots renowned for their show of cherry trees, originally brought from Yashino, and from the banks of the Sakuz river in the province of Hitachi. It is said that cherry viewing firnt became a popular amusement in lede towards the latter half of the seventeenth century. From that time all classes of the people. from the two-sworded samurat to the small tradesmen and menials, participaled in the enjoyment. The green sward beneath the cherry treces was crowded with merry picnic parties of all classes, screened off with low colourect curtains. Ont- fivourite resort, called Asukayana, at Oji. is often spoken of as the mot Yoshino. It is a high, grassy bluff, overfooking as extensive plain on one side, and sloping down to the road of the Oji village on the other. The eminence forms a park of cherry trees extending down the precipitons sides of the hank, so that the pedestrian sees the pinky white blossons againat the blue sky aloove him, and below him the pearly gray of the: blossoms in the shadow of the cliff.

Koganei. snme half day's ricle from Tokio, is perhaps the most attractive spot for secing the double cherry in full bloom. Here a fine avenue of these flowering trees extends upwards of two and a half miles alongr the aqueduct which ennveys the water of the river Tama to Tokio, It is said that they were first planted immediately after the completion of the aqqueduct, by command of the Shogun Voshimune, in the beginning of 
the eighteenth century; with the idea that cherry trees had the virtue of keeping off impurities from water. For this purpose ten thousand trces were brought from Yoshino and from the banks of the rive Sakura: Jut the number now remaining has dwindled to only a few lundred.

In the old temple grove, now a public park, at Uyeno, there are a number of fine trees of the single early-blossoming kind, called by the Japanese Hygan-akkwra, among which are some magnificent specimens of the weeping cherry. This latter species hats pendant branches, clrooping like the willow, and bears single white flowers, but no fruit: and in this respect it is an exception to the gencral rule, that the trees of single blosson bear fruit whilst those of doulse blossom are fruitless. The fruit of the Japanese cherry tree is, however. at its best, insijid and worthless. These trees at Uyeno are said to have been planted by one of the Tokugawn Regents in imitation of the hills at loshino: they are all of majestic size, and present a gorgeous sight in April, with their pale pink blossoms seen partly ayainst the bluc sky, and partly against the rich foliagre of the pines and cedars which surround the golden slirines and eenotapls of the Slogguns, The single-blosson trees at Goten-yama, a park in the suburb of Shinagawa, form a heautiful sight early in April.

The most popular resort in Tokio is the cherry avenue at Mukojima, extending for more than a mile along the banks of the river Sumida. Here the trees lack the grandeur and natural beauty of those at Uyeno, and lave no surrounding folinge to set them off: but they are mostly of clouble blossom, and bending with their weight of flower, -looking almost artificial in their luxurious fullness, - present a most imposing sight. This sput is frequented by the gayest holiday makers. Winc clrinking is considered essential to a proper enjoyment of the scene, and crowds of pedestrians, bearing their gourds of ricrwine, make such resorts merry and boisterous with their carousals. Other visitors, of a richer elass, inctulge in the prospect of the blossom-laden banks from roofed pleasure boats, accompanied often by a gay gathering of singing and dancing giris.

The season of this flower is one of high winds, and the soft petals of the full blown blossons fall like snow tlakes covering the pathways beneath. This simple fact is not without its attraction to the Japanese, who make nuch of the falling cherry petal in their poetry and other arts.

\footnotetext{
"No man so callous but he heaves a sigh

When o'er his head the wither'd clierry fowers

Come flut'ring down. Who knows? the Spring's soft show'ze

May be but tears shed by the sorrowing sky;"-Chssuestus.
} 
The eherry trees in blossom. seen at night by the pale light of the moon, form alse .nother great attraction, Focakma or Night Cherry Flowen being included as one of the sights of the year. The river Lanks at Mlukojima and even the formal avenues of the Voshivara at Asakisis are croweled after sunset, in the cherry season. Cherry viewing at Mluknjima on the banks of the sumida river js illustrated in Plate III.. which represents excursionists ascending the banks from a pleasure boat. 


\title{
SUMMER FLOWERS.
}

\author{
MTSTARLA HLOSSOMS.
}

\begin{abstract}
Nive of the arlieat flowers of the Japanese Summer which attracts the pleatsure (i4) secker, is the wistaria, bloming in May, soon after the cherry blossom has fallen. 7.T. This stalwart flowering creper is reared upon large trellises, arranged to cover long Q5. walks, bridges, or arbours, in pleasure grounds and gardens, I favourite position is one sheltering an open gallery. which overhangs a lake or stream. In the precincts of the popular temple at Kaneido, in Tokio. close to the famous Recumbent Brayon plum trecs there are wistarias of magnificent size, bearing blossoms which lang in rich purple trails from two to thrce feet in length. Wide rustic galleries, in connection with matted garclen sheds and galleries, extend over an artificial lake stocked with gigantic gold fish, and the wistaria trelliscs form an extended covering overhead. A belicf exists that this flower attains great size and beauty if its roots are nourished with the rice-wine of the country. and there is, at Kameido, a tree producing specially fine blossoms, at the base of which visitors are accustomed to empty their wine cups. Fine specimens exist in various parts of Japan, bcaring clusters over three feet in length, among which may be mentioned one at Noda, in the province of Settsu, called the Chitosc, or tree of a thousand years.
\end{abstract}

The wistaria of purple blossom is most conmon and at the same tine most entecmed, ranking higher than the whice hind, which is resardent as alnormal. This is an exception to the prevailing custom, which places white before other colours in blossons of the sance species, and especially proseribes purple llowers as associated with mourning, and, therefore, unfit for felicitous occasions. In various designs the pheasant is shown in combination with the purple wistaria.

The tiew of the wistaria in llower as enjojed in olden times by ladies of rank is shown in Plite IV: 


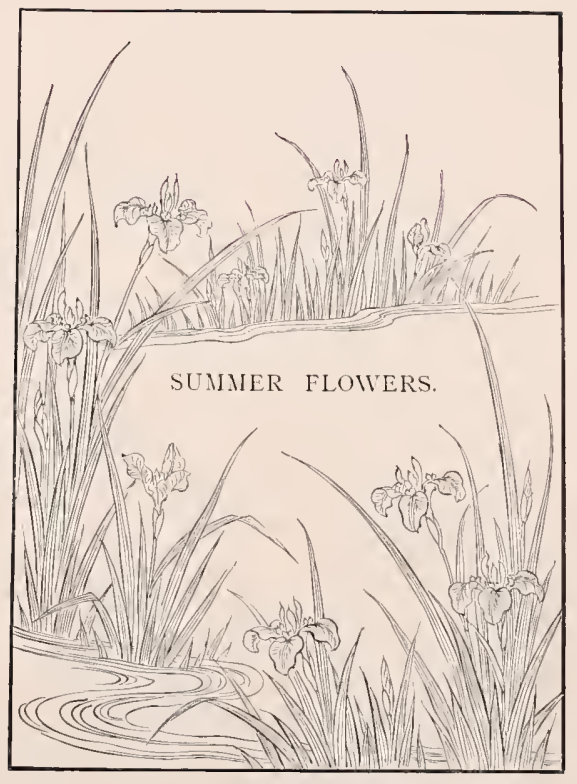



PLATE IV.

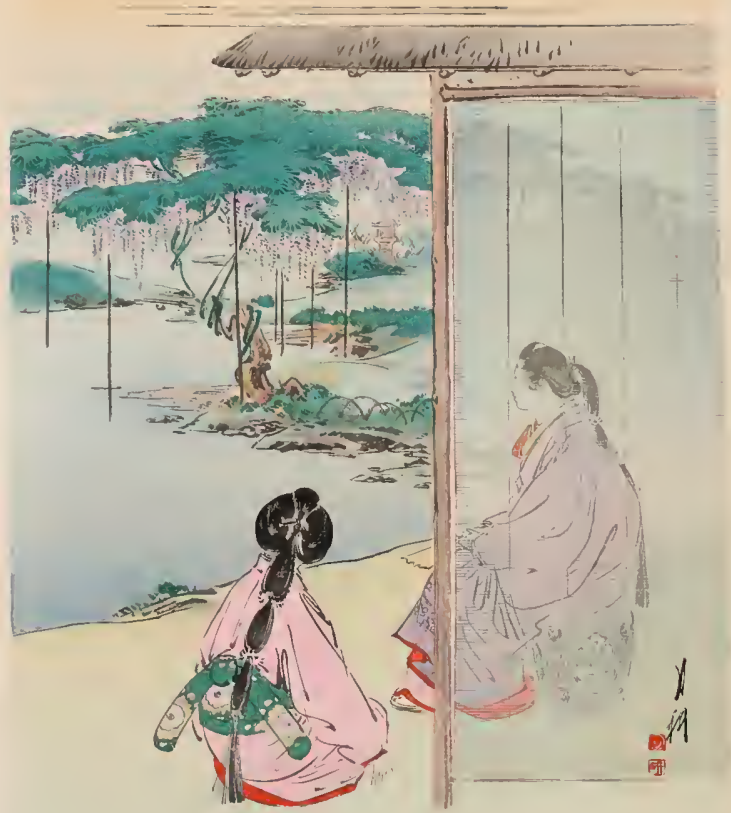

WISTARIA BLOSSOMS IN A NOBLE'S GARDEN, 

PI.ATE V.

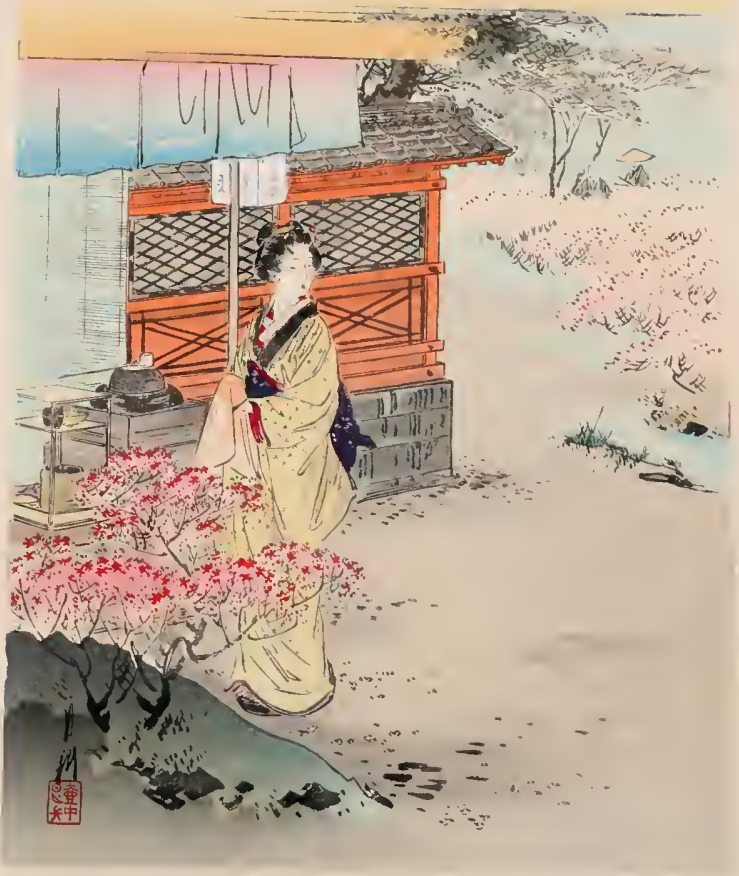




\section{IZALIAS.}

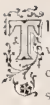

IIE azalias commence to flower about the same time as the wistarios, and display a variety of colours of most brilliant huc-numerous shades of searlet, crimson, orange. cream, whitc, and magenta--unequalled by any other blossom. The hardy azalia shrubs are abundantly planted on the sides of artificial hillocks and on the slopes of terraces, and a Japanese landscape garden, usually remarkable for its wealth of evergreens and predoninating verdure, never looks gayer than when these bushes are in flower. There are several public gardens at Oktho, a village near Shinjuku, in Tokio, which are planted entirely with azalia bushes of great size and remarkable agre. Thesc plantations date back to the time of the Tokugawa Regents, by whom they were frequented, and the $y^{*}$ are still visited every summer by numbers of sight-seers. One azalia tree at Okubo has a stem as thick as a man's leg and is said to produce eight thousand blossoms at a time, Other places where the azalias may be scen to advantage are,-Ujeno Koyen, Uyeno Okeiyen, Asakusa Koyen, Shiba Koyen, Susahi Benten-no-sha-nai. MIukojima Mokubo-ji, Azabu Shokayen, Honjo Uyebun, Meguro Daikokuya, and Horikiri Musashiya,-all parks or gardens in the vicinity of Tokio. Plate V. illustrates the azalias in the grounds of the Gongen shrine at Nedzu, a suburb of Tokio. In this enclosure is a famous stite of chanbers used for the Tea Ceremonial and much patronized by the public.

\section{IKISLES,}

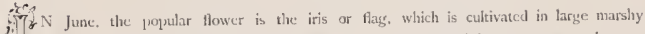
3. If fats near to rivers or lakes. In many gardens, watercel by a stream, a loop or if $y_{\text {S }}$ bend in the water-course is spread out into a marshy expanse, planted with irises, $7_{\uparrow}$ and crossed by fincy plank bridges of zigzag shape. There are in Japran fout distinct species of iris, known by different native names, but the kind most seen is the Acurns calamus, or sweet flag, which the Japanese call Hana-shobu. In the case of displays of iris flowers. a mass of varied colour is delighted in: the purple, white, and variegated blossoms being grown together, indiscriminately, and with little or no attempt at pattern or design. The most noted place for shows of this flower is Yatsubashi, in the provinee of Mlikawa, the 


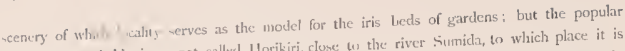
resurt nearest Iokio is a spot called llorikiri, clume th the river Sumida, to which place it is the fasbion to make excursions in pleasure boats carly in Junc: Here the becls which contain Whe fiomers in cvery rariety of colour, are surrounded by elevated grass. banks, detted with smmer-lionses, from which visitors can leok down upon the richly variegated carpet below. Narrow wooden bridiges give further picturesqueness to the scente, crowded in the season with a brilliant throng of visitors, whose pretty costumes alnost vie in graiety of colour with the flowers.

The iris, ats of water plant, is associated in at with the kingfisher, water rail, manclarin tuck, and other water birds.

Plate VI. shows the iris bedis is they may be seen at many places on the outskirts of the city.

\title{
PEONIES AND LOTUS FLONERS.
}

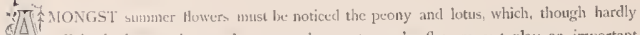
20 sufficiently democratic to rank imomg the mast popular flowers, yet play an important fot part in the art of the coumary. The puony is a delicate plant and is cultivated Fin in long sheltered beds, senerally forning the forpterre to some adjoining chamber. from which its magnilicent blossoms can be viewerl. In the grounds of the wealthy it is subjected to scrupulous care and mursing, in order to produce Howers of enormous size and fulluest, often so liurge and heavy as to need artificial support. It is regardecl ats tle Hower-qucen of China, and is essentially the favourite of the upjer classes in Japan. The prony was first imported into this country in the eights century, and was then chiclly cultivated in the proviness of Yamato and Yamashiro. Even now, the finest specimens in luhio are brought from the neighbourhood of the old capital. Nara. The largest blossoms nholisure as much ats nime inches across. The peony is sometimes called the flower-ofprosperity: : wnother fancy name by which it is know in the plant-of-twenty-days, because it in said to preserve its beauty and freshness for that period of timc. Of the lange trecpeony, called botan, there are ninety distinct kinds, and of the small plant-peony, having
\end{abstract}


PLATE VI.

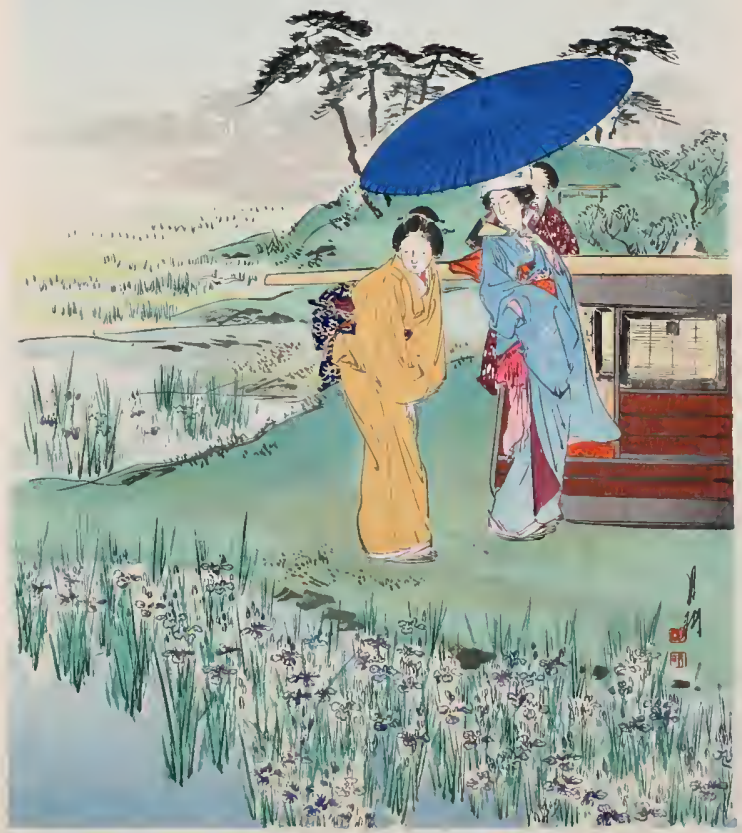

IRIS BEDS, NEAR TOKIO, 

PLATE VII.

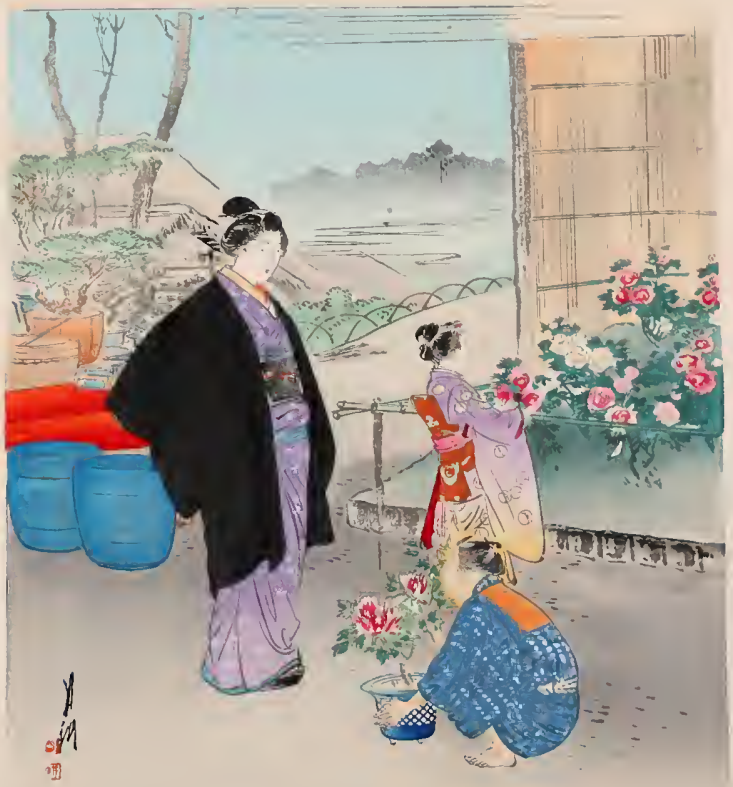

PFONIES AT YOTSLME, HONJO. 


$$
\text { (1) }
$$


single blossoms and called shakwyaku, there are sail to exist five bundred varictio The botan may be inspected at numerous public gardens in Tokio such as the Hanjitsuyen. Unsanyen. Gochituyen and Sendaiyen at Komagrome; the Uyehun. Yokayen, arkt Teigo. yen at Honjo; Daikokuya, Megurn: Taikoyen, Shiba; and Senkayen and Shokayen in Azabu. The shaknjuku is also shown in the Uyebun. Honjo: the Voshinoyen at Kimme. bikifune-dori and the Teigoyen at Mlinami futaba-cho Honjo, Among colours, the red and white are most valued, purple and jeilow specimens, though rare, being less prizesl. This exuberant flower, with its large curling petals, is a favnurite subject for slesign and decorn. tion. Its companions in art are the peacock, the golelen pheasint, and the shistri, a kirkl of conventional lion derivet from Chinewe thesigns: in stch company it forms the constant decoration of temple and palace walls. A show of peonies in the sereened and sheltered beds in which they are grown is represented in Plite VII, takin from the garelen of the Uychan. lotsune, Honjo.

The lotus is closely connected with the Butdhist seligion, and is, therciore. associated in the minds of the people with spirit-land. The lasea of temple grouncls, especially those dedicated to the water goddess Benten, are frequenty planted wiliz lotuses, The laks: Shinobazu at Ljono has a fine displiny. The fine wicle moats of the Tokio Castle abound in these water plants, which impart to then considerable bututy in the summer season. Wherever undisturbud pools and channels of muclely water exist, the lous is to be found, and even the ditches besiste the railway connecting Tokio with the port of Yokohama are rendered kay in the summer by the lotus flowen in blonm. As the peony is saicl to be the national flower of China, so the lotus is regariled as the national flower of India, the source ind centre of Buddhism. It is there [ere considered ont of place as a decoration for occasions of festivity and rejoicing, lout it is comstantly used for obsequies and other sacred ceremonies, The lotus serves as a suitable theme for religions contemplation, and is therefore the favourite flower of monastic and temple retrents: the best displays are to be seen in the lakes of the olcl temple groves of Kioto and other cities. Growing out of the muddiest and most stagnant water, its keaves and fowers are always fresh anil clean; although it is particularly sensitive, and quickly withers if hrought in contact with any of the fertilizers by which other plants are nourished. This purity which the letus maintains anvid surrounding firth is nentioned as onc reason for associating it with a religious life, A well-known bonk of Buddhist precejts contains this text:- "If thou be born in the poer man's hovet, but hast wisdom, then art thou like the lotus Hener growing out of the nud ".

The white lotus Hower has it powerful and sweet profume, but the reat kind. though more handsome, produces but little scent. There is a species called Golit-thread- 
Larue, its red blossoms being marked with yellow lines; and a very handsome Hower of deep crimson colour may also be found. The Inclian lotus, which is sometimes to be seen in Japan, has a large double flower. of red colour. which never closes day or night, but falls off after five or six days. The blosernis of the ordinary kinds close after midday. The leaves of the lotus are almoit as much esteemed as the flowers, their broad curling surfaces of deep green and emerahl presenting a beautiful effect in the lotus ponds, and forming a Gavorite subject for the jainter's brush. In designs, the mandarin duck ant other watus fowl are represented with the lutus. 


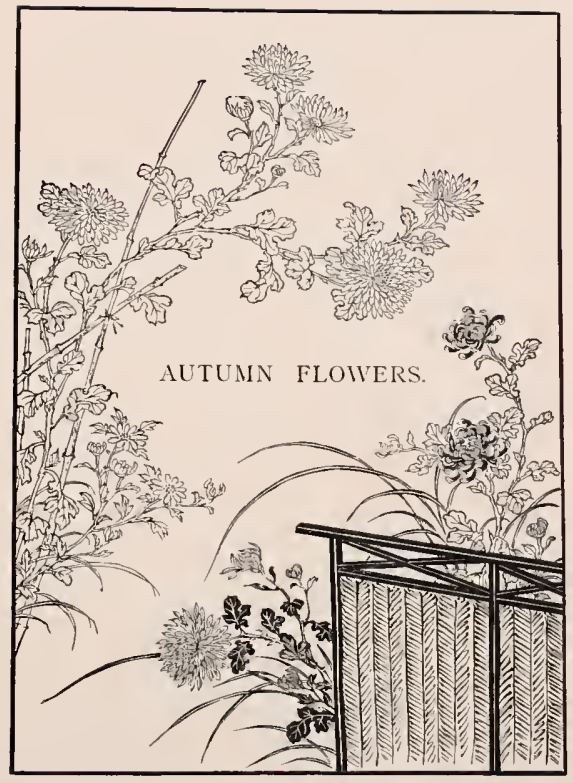





\title{
AUTUMN FLOWERS.
}

\author{
CHRISANTHEMUMIS.
}

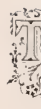

HE chrysanthemum is the principal flower of Autumn, and the triumph of Japanese. floricultural skill. Specimens of remarkable varicty in form and culour of blossom are produced in the gardens of the mobility. The flower of the chrysanthemum. in its most handsome form, loses its disc-like character, and presents a cumbination of long oval petals. partly extended, and partly curling inwards, exhibiting in contrast the different tints of face and back: whilst, in its most eccentric and artificial shape: it assumes the character of a confused mop of tangled thread-like petals, morc curious thun beautiful. The florists aim at producing an extraordinary quantity of blossoms upon one. stem, reaching often to the number of several hundreds. The varieties of the Japanese kiku are nunerous, including not only those species classified as chrysanthemums by European botanists, but many kinds of l'yrethrum, Aster, and other genera.

It seems that the wild chrysanthenum, of small flower, has always becn indigenous to Japan, and held in considerable repute from every carly times for medicinal purposes: in which connection early records state that large quantitics of the ycllow kind were yearly sent to the Imperial Court from the Southern provinces. The lange cultivated flower, however, is said to have been imported from Corea or China and first planted in Japan at Hakata in the province of Chikuzen. At this tine five colours were known, described as blue, yellow, red, white, and black, the torm black probably referring to a dark purple colour. Origsinally these plants were reproduced by means of slips and cuttings. but now the seed is employed, which is said to give greater varicty of blossom.

The chrysanthemum is sometimes spoken of by foreign authors as the mational Hower of Japan, a rank properly belonging to the cherry blossom; and this mis-conception is probably owing to the former being used as one of the crests of the Imperial House The flower has always been much honoured by the Court, and as early as the tina of the 
Emperor Heizei, in the ninth century, sarclen parties wcre held in the Palace for the purpose of chlobating its hossoming time: just as. at the present day, a yearly chrysanthemum show tikes place in the Imperial grounds. These ancient celebrations seem to have partaken of a truly. pastoral character. the courtiers wearing the plucked blossoms in their lair, drinkins winc, and composing verses upon the beauties of the Howers. The motern chrysanthenum displays in the Palace gardens are more like our own flower-shows in the social conventionality of their arrangements; but the nunerous variety, of every imagrinable colour and profusion of shape, arranged in long open rustic sheds, forms a brilliant and imposing scene hardly rivalled by any flower-show in the workl.

It a recent clisplay in the Imperial grounds at Akasaka there were a hundred and sixty varictios of hlossom exhilhited, each bearing a fancy name of its own. Some of these names are so perctically sugsestive of the form or colour of the Howers that the enumeration of a few of them trill not $1 x$ : out of phee :-
Chi-kin-gi.
Gin-sitkai .
Tersestrial cilobe-a large slobular ydlow Hower.
Isti-zrasum! Silver World-a flower of pure white colow:
Ysuliti-no-fonin. Thin Mlist - a white flower:
jii-hi-kag
Tiema-sudare
Companions of the Moon-a white flower.
Ifatsu-yuki.
lkume-gataut
Aivari-bi.
Shadows of the Exening Sun--a flower of ciull red colour.
Isa-hi-wo-nami
The Sereen of fiens-a Hower of erange red colour.
- Wir-no-soriz.
The First Snow-a pure white blossonn,
The Basket of Flowers-a rich red flower.
Beacon Light-a red flower.
Waves in the Morning Sun-a reddish Hower.
Shigarami. . pink).
Ciarden licnes-a Hower the edolir of the wistaria blos- som (hvender colour.)

- Asa-negani

Hoski-dinki-yo .

Dishevelled Hair (lit. in morning sleep) -a flower of tangled petals.

Ihaski-no-hikari

Starlight Night-a white Hower.

himi-no-nsçumi

Star's Brightaess-a pale bluish flower.

Juki-no-askilit

Tishiz-Mo-kasa.

lilessingrs of Hajesty-a pale pink Hower.

Ogont-no-nishili. Snowy Morning - a flower of pale pearly pink colour. Moon's Halo-a flower of orange red colour. Golden Brocade-il flower of golden yellow colour. 
PLATE VIII.

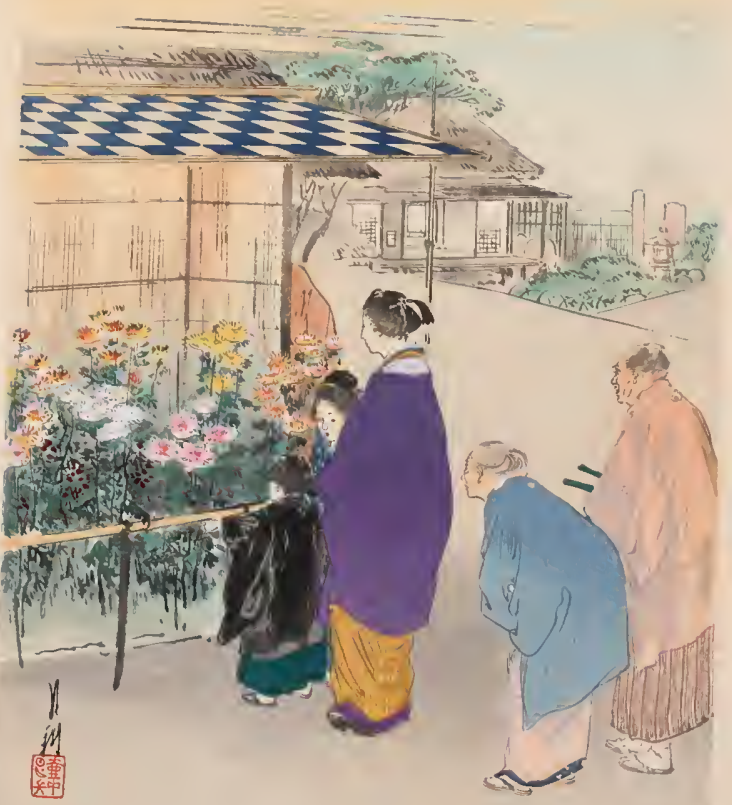



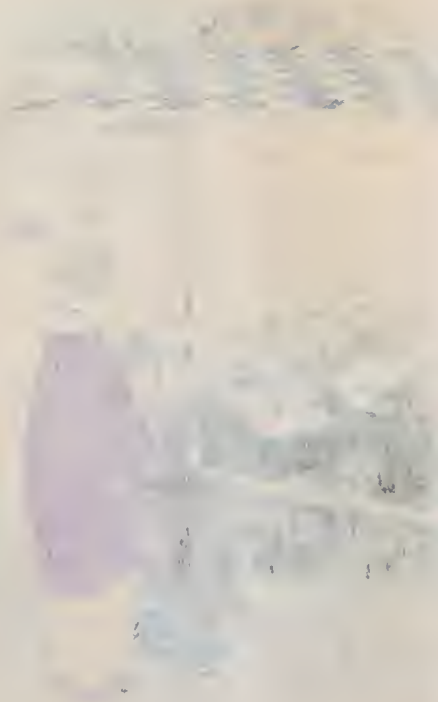


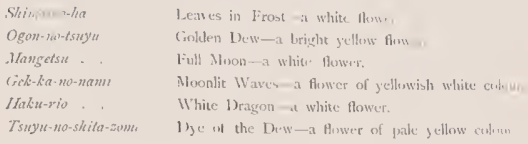

There are sail to be in Japan two hundred and -ivty-niz. colons vurietu of the clurysanthemun, of which sixty three are yellow, eighty-s-sen white, thirty-two purple, thirty red, thirty-one pale pink, twelve russet, and fourteen of mixed colours, A fincy previals that in this flower the same tint is never exactly reptrolucesl, and that it thus suggersts the endless variety of the human countenance. Blooming hinger than mont fluwers, the chrysanthemum has come to he associated with hongevity. In the prowince of kiai. ot hill. called the Chrysanthemum Mount, overhangs a river of dear water, into which the prabs fall, and a belief exists that long life is assured by drinking the water of this stream. I favourite motive of decoration, which may be seen in numerous conventional designs, is the: clurysanthemum blosson floating in running water. A custom also sturvives of placing small blossoms or petals in the cup during the winc-trinkin:s which takes place on the festival of the ninth day of the ninth month.

The ordinary varictics of chrysanthemun are to be seen in great abundanee in the strect fairs during the Autumn months. Dango-zaka, in Tokio, is a fevourite popther resort during the ehrysanthemum season, but here the flowers, mostly of the smaller kind, are used artificially, modelled into groups of figures and animals representing historical subjects. seenes froms populitr theatrical performances, and even the battle seenes of the China-Japan war.

The clarysanthemum is associated with the cranc, the royal hird of Japan, I'late VIII. illustrates a display of clarysinthemums in a giualener's groumls at bumei. 


\section{THE SIVEN PLANTS OF AUTUMN.}

inf $1 \mathrm{HE}$ paucity of important flowering trees and plants in Autumn. has led the 2. "Sapanes to make much of cercain simple plants, comparatively insignilicant in 2. If themselves, but gathering importance and interest in combination. As has alruady "Yw been pointed out, almost etery month of the year is associated with a special blossom, and the calendar would therefore not be complece wichout a reference to these flowrs of the late Autumn. These seven plants are: the lespedezat the morning glory. the Eularia japomion, the Iisteriana sillosa, the Iaterianta officinals, the Pueraria thunbrigiama, and the carnation. Perhaps the fivourite of chese is the lespedeza, or lusis clover, of which there are several kinds, some having pink, some white, and ochers yellow flowers. Growing wild on grassy moors, it is assuriated with wild horses, deer. and the wild boar, together with which it is otoen depicted in trarious designs. The deur is specially associated with the Autumn time, and represented also with other Autumn flowers and with the reidening maple. The seven Autumn plants are grow s togrether in the

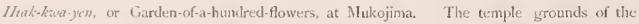
Ihyg-dtert, near Kaneido, arc larnous for their show of lespedeza flowers. Displays of various kinds of convolvuli or morning glories, trained in pots over skeleton framework representing rustic structures, are also to be seta at the various gardener's nurseries at Jriga. one if the suburbs of Tokio, by those endhusiastic enough to reach the spot before six ocluck on an Autumn morning. Fancy flowers, less than half an inch in size, in clusters. and shaped like a butterfly orchid, and other strange varieties, nay be seen. Within the last year or two the culture of convolvuli or morning flories has become very popular, and they may be observed before many dwellings in the city on quaintly designed frames. Mukojima.

Plate IX. illustrates the seven plants of Autumn as grown in the Hiak-kitent-yen at 
PLATE IX.

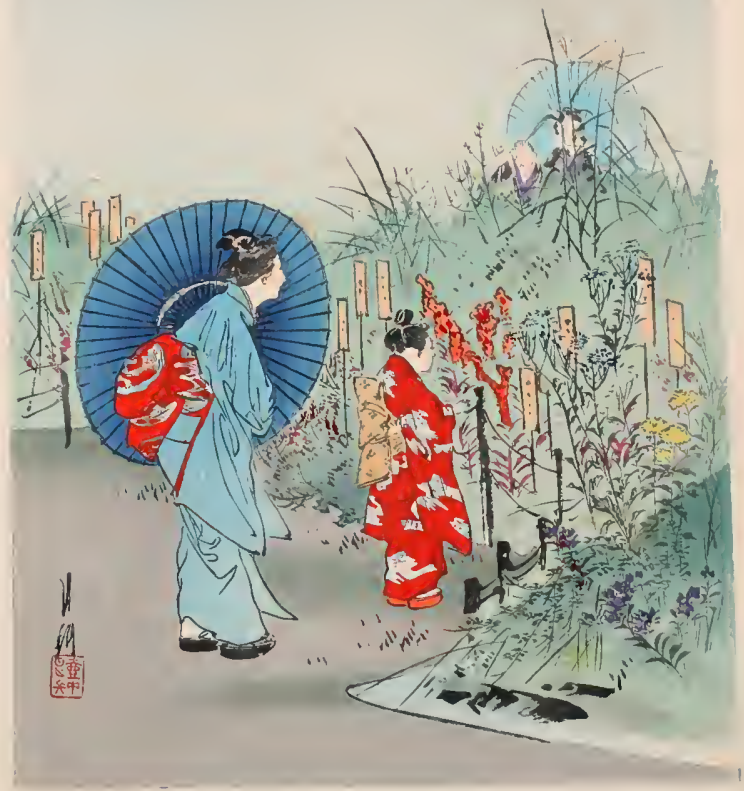




\section{IPI.ES}

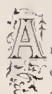

NOTICE of the floral festivals of the year as observed in Japan then +1 amo mention of the maple,-for the reddening latif of the majke, like the fuliag of many other blossomless trees, is regarded as a flower in Japra. The rich tints of the

changing leaves of certain deciduous trees, hardly distinguishable from the colouring of blossoming shrubs such is the azalin, form a furourite object of attraction dluring the Autumn months. The native term momijt, which in commonly tramslated mople, is, strictly speaking, a general name applied to many trees which rechlen in the Fall, (ff the maje: itself, there are many varieties, distinguished both by the form of their leaves ancl the torne: of their changing colour. No garken is consickercel complete without its group of such reddening trees, placed luside some artificial hill towarels the Wiest, to receive arklitional splendour from the setting sum. They are planted on grasy slopes and in valleys, with the object of bringing into one limited prospect the red and grolelen tints in which the natural scenery of the woodal hills abounds. The grand slopes above the river Oi at Arnshi-yama, noted in the spring time for their show of cherry blossoms, present a fine display of scarlet maple fuliage in the Autumn.

Ae Ko-no-thi, a fanous prominence commanding a view of the whole plain of Tokio, there are some masnificent miple trees, noted for their cnormus sier. A synt called Tatsuta, in the province of lanuto, is renowned for its line specimens, whith line the banks of the river, and are in full glory about the end of October. At (hi, a sulurh of Tokio, the slopes of a matural grten between the hills are planted with thick mines of these trees, forming a most ronantic spert, where, from the gullerics of a cuntic arbour, the sight of the foliage in all its burning splendour may be enjoyed. Shinagawa ant Maruru, other well known spots in the vicinity of the capital, have alsu grood groups of maples which attract many sight-seers. I'icnicking and mushroom gathering are pastimes which accompany the viewing of the maple.

In the poents and pictures of the country the maple is associated with dieer.

\footnotetext{
"IIow full of surrow seens the Atatumn I when, In soltary rambles sluwly strajing.

And the russet lohase of the glen,

I listen to the lunely siag a sind baytog."
} 
11. Sur ins view of maples in the gien called Taki-m... an, at Oji is geven in Plate $X$. In the distance may be seen the rustic sheds from which visitors enjoy the prospect of the scirlet folinge: whilst in the forcground is shown a pirl rcacling some of the verses attached to the lower branches of the trees. 
PLATE $X$.

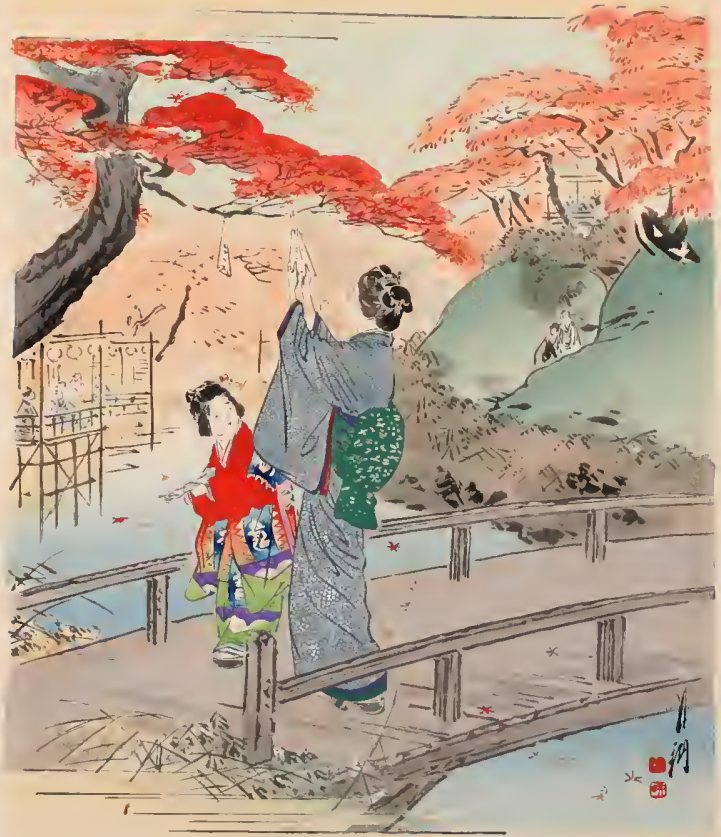




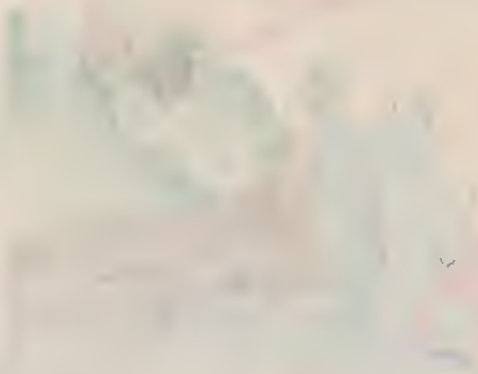




\title{
ARRANGEMENT OF FLONERS.
}

\author{
INTRODUCTION.
}

\begin{abstract}
$\rightarrow 20<$
4. $17 \mathrm{fTH}$ the Japanese, the arrangement of cut flowers in vessels of various kinds law 2 3 becone a decurative art of considerable refinement, compared to which IVestern the methods of Boral composition have the appearance mercly of haphaard combinattions. The houquet, wreath, and garland, all depending for their beaty upon the close massing of blossoms and greenery in soft and luxurious confusion, hear no resemblance whatever to the more austere and open compositions of the Japanese which belong entirely to a different phase of art. The fact that many of the most charming flowers of the country are those of trees, the blossom-chd twigs of which it would be ilifficult to arrange in closed and rounded masses, may in some manner explain the minn lineal character given to foral designs; the same treatment is, howcver, equally applied to flowering plants and grasses which would lend themselves far more casily to the European method of grouping. The reason for the peculiaricy of treatment toticeable in these flower arrangements may rather be sought in the Japanesc manner of observing and enjoying floral nature. Whereas the Western amateur devotes his attention mainly to the blossoms, the Japanese lover of flowers extends his admiration to every strikins feature of the plant or tree producing them. The rusged nature of the plum trunk, with its straight, stiff shoots, or the graceful sweep of the branches of the wceping cherry, are to him inseparably associated with any beauty which the blossoms themselves possess. The lines of branch and stem, the form and difienent surfaces of leaves, and the distribution of buds and blossoms, all reccive an equal share of attention and all play their allotted parts in designs. It may be said that the art under consideration is based typon a representation, more or less conventional, of floral grouth; and, principally for this renson, the compositions are made to assume an open character in which the forms of branches, stems, leaves, and flowers are all clearly and individually expressed.
\end{abstract}


The vernacular term hana, translatable is flewer, lins, in the art of floral arrangement. a much wider signification than its nearest Englivh equivalent. Among the so-called ferams of the seasons are inchided certain evergreens and other flowerless shrubs and treen. rnmwe of these holdingr very high foral rank. The pine and bamboo. for example, both 1 mupy a very inyportant place in what are called flower arrangements; also the maple with its reddening leaves is ased as one of the principal flraters of Autumn.

In the choice of material, seasonableness is one of the principal points kept in vicw. The luxurious taste for chniceness, as implying rarity, is dianetrically opposed to the zules of the art under consilteration. Flowers blooning bcfore or after their proper season are, with very few exceptions, rejected for Japanese floral compositions, such designs being In a manner intended to express the particular period of the jear. April blossoms, useal in any other month. wontl appear to the flower-artist as incongruous and out of place as wintes clothing worn in summer-time, It therefore naturally follows that a proper cultiration of the floral art demandis a thorough acquaintance with the nature and growth of all trees and plants cmployed: and, in the case of those common to several months, a close observation of the varying characteristics of the same plant cluring different seasons. The thas or iris, for example, which is common to different months of Spring. Summer, and Autumn, has a peculias bend and vitality in its leaves, and a different length and vigour in its flower stens, during the various periods of its growth; and these distinctions are all kept in view when this flower is employed in compositions.

The natural locality of proxluction of trees and plants; whether lake or river bank. motntain, or moor: greatly influenees the claaracter of the clesign employed. To arrange a water plant in the same manner and with the same surrounclings as a land plant woukd be considered a great violation of the rules of appropriateness. Not only are blossombearing trees and Hutvering plants treated as perfectly distinct in character, but ninor divisions as to Jocality of production are uften observed in both. Among planti a distinetion is made between ordinary land plants, forest plants, mountain plants, and water plants; and among trees, lanel trees, forest trecs, and mountain trees are distinguished in
certain cases.

The necessity for a proper familiarity with the nature of all Howers used in plampositions is one reason strongly urged against the employnent of rare or litule known plants, however beautiful they maty be. The Uic of wild Hewers, onily known to the lnitanint, as well as rare forcign flowers the nanges of which are not familiar to ordinary
folk, is prohibited, unless the artiat has previously made hinself perfectly acquainted with all 
the natural characteristics of auls flowers. As une expon it of sit art hat quisatly expressed it. the artist must be thoroughly imbued with a sympathetic fecling for tlu. character. labits, virtues, and weaknesses of the menlyers of the floral kingslon from which be secks his material, till he possesses almost the same love and wenderness for their qualities as for thost of living lueings:

Preliminary to a study of Japanese Floral Art it is mecessury to have sume acquaintance with the principal flowers employed. These flowers are cnumerated in the following page's under the heacls of the different months to which they belong. In consequeme. of such a classification many Howers common to several months are repested. It must bx remembered that according to the old cakendar the exmmencenent of the first mombliwhich was at the same time the beginning of the Jaynesest Spring - was about thisty clayn laer than the first of Jantary. The acloption in late yeurs of the Gregoriat calenclat hats therefore rendered it improssible to conform it the present day to all the rules bird down for the selection of flowers for special uccasions. Such of the olkt lete-clays as are nuw observed, are pushed back one month or more in time, and the flowers originally fiacel as appropriate for their celcbration are often unavilable, of recourse has th be made to premature or forced specinens. The following classification is according to the ohl calcndar, existing when the whole theory of the art in qutestion was established. 


\title{
FLOWERS ACCORDING TO THEIR MONTHS
}

\author{
(OI_D CALENIDAR).
}

Agatsat the Japarese names in the following lise of flotwers certain distinguistring signs are placed :-

* Stands fur those trees or plants which are termed Lining Flozws, being farticularly charactenstic of

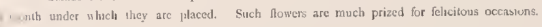

+ Distunguibies the Early Flowerngs, - flowers which are in advinee of their proper seasun in the month inder which they are flaced. These bave also their appropriate use in floral compositions.

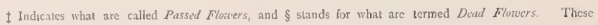
names hase relerence to flowers which are late or passed in month or season, belonging properly to earlicr monthis. The use of such flowers is forbidden for most ceremonial occasions. There exists also the ternu Vinlgar byouses, applied to wild plants, or to those of very common charucter which pussess no fancy name; and the employment of fluwers inclided ander this head is not permitted excent in the hunds of the must experienced proilessors of the art. The use of cereals is also to be avoided.

\section{FIRST MONTII (PRESENT FIBRUARY).}

\begin{tabular}{|c|c|c|c|c|c|}
\hline JAFANESE NASIE. & 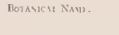 & 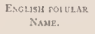 & JA+A\ESF NAVE: & ButAY:cal Navg. & $\begin{array}{l}\text { ERCLISII HOTULAR } \\
\text { A'AME. }\end{array}$ \\
\hline Pukujust & Arlonis anturestse & & "kengo & I orsythiti suspenst & \\
\hline Shunsai & Naicisous tractia & Saruisis & Tulatis: & Camclina swanter & Camedtia \\
\hline - Uyensuse & Lisho-jermuna fullingter & & "Dlurozaki-Nomo & & Hut house pesch \\
\hline Hiltulas & Irunse muase & Wlute fluas & - Obai & Jastainun sichollianuin & \\
\hline SYansge & stos pронис & Willowe & "Kinscenlwa2 & CJentula ofleciralis & \\
\hline SKantqul,a & 1'yrethruin siatess: & $\begin{array}{l}\text { Winter chrysus. } \\
\text { themisa. }\end{array}$ & -Chiootima & Fosen indes & \\
\hline "ratuken & STlers pgunila & & $\begin{array}{l}\text { 'Mansalus } \\
\text { SKotai }\end{array}$ & Hatnametis jayonica & \\
\hline & & & ŞKotai & Chimonanthus tragrants & Clunese plam \\
\hline
\end{tabular}


SECOND MONTH (PRESENT MARCH).

\begin{tabular}{|c|c|}
\hline Jatings Naห & TOTANLEA NAMT: \\
\hline $71103, u-b s i$ & Pronus mume flore alba \\
\hline \multicolumn{2}{|l|}{$\div 116-4 s$} \\
\hline *nlai & Jasmum sebohidonum \\
\hline "Ko tn" & Iromus atume Arese ros \\
\hline - Hhponesokuma & Trunes subhirtellx \\
\hline Tisurin & Prunus persica \\
\hline Primations & Simalincus racemosa \\
\hline "Kevanco & Dicentre spectalintis: \\
\hline 4 Arums gike & Irngeton thunletgi \\
\hline ZKinsenkwa & Calentials ntheinabs \\
\hline t1 Iaru-giku & Cheysanthessums cormiafium \\
\hline •Ilotei chiks & Marahus sterilis. \\
\hline - Temnanshr & 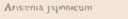 \\
\hline to bh: knbone & Nughar jnomevm \\
\hline "Reninn & Jinegytixa sutpenes \\
\hline - Anra & Propus arrucniaca \\
\hline †'llibycezahura & Prunes |sculo-echisuy \\
\hline † W.tsmabuhi & Kerna japonsei \\
\hline THitsugisn & Nymplites tetranom \\
\hline - Niv s-bme & Truinus japoner \\
\hline -Wase-zikburs & Trunus pseurlo cernsus \\
\hline -Nisia-zahurat & Trunue pscudo-ocrasus \\
\hline tEnisibia & Cytisus scenporios \\
\hline +Nokuremge & Misgnolia \\
\hline tSumo & Cжxalyutu mpan \\
\hline tVohsthwi & Mfyner rulin \\
\hline
\end{tabular}

\begin{tabular}{|c|c|c|c|}
\hline $\begin{array}{l}\text { EMULII TOFTLAR } \\
\text { NAYR. }\end{array}$ & JAFANESE RAMT. & BotANiCAt, Nive. & $\begin{array}{c}\text { ENGLLIS POPULAR } \\
\text { NAMF. }\end{array}$ \\
\hline White ptum & Hiakunage & Whi-lotemitren usctenizhis & Aralen \\
\hline \multirow[t]{2}{*}{ Rul / ath } & +Tsulful & Rhoaloctendron malicum & Arien \\
\hline & thanto & Fyrus spectabtlis & \\
\hline \multirow[t]{2}{*}{ Ret pluner } & 50 lass & Jasenipum sicloilatisoum & \\
\hline & - luaran & Acpilestan tuntt & \\
\hline \multirow[t]{5}{*}{ Tale pets } & - Majinen: & Mapnyer rherex & \\
\hline & FCTguistion & 1.ilbojkrmum inlanger & \\
\hline & *Yukivarison & Arcmenc lieftirtict & \\
\hline & "kiaranemalaka & Alisma plantago & \\
\hline & -Shunrar & Cymbadium marns & \\
\hline \multirow{2}{*}{$\begin{array}{l}\text { Sigring, elerysos- } \\
\text { themum }\end{array}$} & - Kabuclir & Magnolis katuse & Slipunis \\
\hline & \$Tsuliah & Canicllan jupunies & Lamselln \\
\hline \multirow[t]{4}{*}{ Duminon } & + Nixstii & $\mathrm{F}_{2}$ ros usernentis & Mar \\
\hline & Astumoftits & Fyous Inilors & \\
\hline & -Ringo & Tytus malus & Anple \\
\hline & Asetin & Anilromals јручтыст. & \\
\hline \multirow{3}{*}{$\begin{array}{l}\text { Arricol } \\
\text { stragle chatry }\end{array}$} & - Sentastapy & Theersopisis falipery & \\
\hline & - Koraj-ghos & & $\begin{array}{l}\text { I कालa chry-a } \\
\text { thethum }\end{array}$ \\
\hline & tBate & 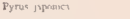 & \\
\hline Garder plum & ¿Mfanake & Vamamelis upobicy & \\
\hline Early cherry & -Choatiun & Rnsa indica & \\
\hline \multirow[t]{2}{*}{ Gardea cherry } & -Wosotegush & Ilemerocallus flava & \\
\hline & - Itulorí & Tolygnanum cuspi latum & \\
\hline \multirow[t]{2}{*}{ Afagnolin } & -Sumirc & Viola patriair & Vyolet \\
\hline & •पामा० & Freniculun vulgare & \\
\hline
\end{tabular}

THIRD MONTH (PRESENT APRIL).

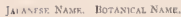

*Haha in Pruacs persica flore alho

- Lisu-to

- Ningro-momo

- lis in

"Niven-aimarar

- Hosumomo

- Ko ta

viendist momo

tRi.10

-Nashi

-rkingo
Trunus toiforit

Pyrus uskuricnsis

Pyrus nalus

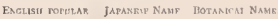
NAME.

White percls.

Punk peact

Thach

Red reach

pesch

Peach

Red peseh

Rel and white perch

Pear

Apple
- Vamabuha Kicrria japonicis

\#Reagin I oreythas retgenes

Kolusha Magnolia holvos

Anzu Inumus armenisto

Jinru-pite Chrysantbemum crimenariom

Jacholes

Euinero

hiome-zokurs Nwa-zhtur:

Suno

Wase-zahura!

Calimitic elicuoror

Spotien thunierges

Prones pesulin cerasus

Cxalpina sappare

Pruthus pecustreerasus

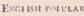
NAME.

Magnolia

Aprent

Spragiz chryean

thicrium.

Dyphine

Kinel of orchil
Gunlen cherry

Lurly cherty 


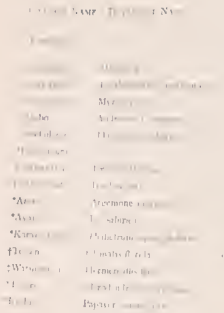

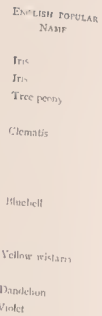

\section{FOLRTH MONTH (PRFSFNT MAS)}

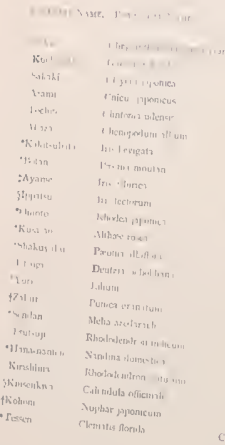

\begin{tabular}{|c|c|c|}
\hline 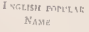 & JATINEER NiUtE. & - Totanirat NaME \\
\hline \multirow[t]{6}{*}{ Itrysanthenente } & - Nidangohi & Devotoir sicboldhanas \\
\hline & thirass: & Seturis kamiscliaricuin \\
\hline & PBiptien & Pipparer rlicens \\
\hline & tEuto ? & Seinus Leustrue \\
\hline & |Cuastinta & Cylusus scrymarnus \\
\hline & 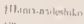 & Diantfius Myonicas \\
\hline fi. & +Tsumganem & Campranuls punctiati \\
\hline $10-1+1+42$ & - Biyapre & Hyperieun chir ense \\
\hline Jit & †kouleman & Siŕras cantoniensus \\
\hline 1 & :Sumir & Cosalpinas sinpon \\
\hline & 5 kighush & Nagnolin hotius \\
\hline & t5han 1 & Iris juotilet \\
\hline 1,16 & thfohurent & Dragntolia costajucus \\
\hline & - Shiundsuhe & Spunce juponice \\
\hline Leits & Senitallerge & I heragegsit fabacca \\
\hline & *Slaran & Bteria ligncosthen \\
\hline & eTeqpa-yus & 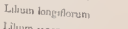 \\
\hline$\Delta x>\downarrow$ । & -3xea-yuri & Lillum piporicua \\
\hline & "1time-g uri & L.hum cancalor \\
\hline & ILriknatar & Senccio yporaicus \\
\hline & Cintiorti & Funki, nvala \\
\hline Contia & $\begin{array}{l}\text {-Kuningayeso? } \\
\text { Astuniorien }\end{array}$ & 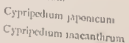 \\
\hline
\end{tabular}

ExGetsai mimaz

None

IHucheil

\section{MTagenaldit}

Ins

Ifignoliz
Lity

Laiy

I.lly: 


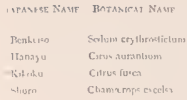

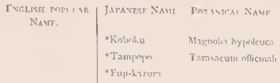

Fin Liat rost tak NaviE.

Mreariot

Onmlelion

\section{FIFTH MONTH (MRESENT JUNE).}

\begin{tabular}{|c|c|}
\hline Jamiv5Y NAWF & DotANEAT PAvir \\
\hline *Kiku & Clorystalhemun eosnnariun \\
\hline 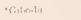 & Tunkia ovits \\
\hline F⿻ Chor aII & Elictia liyscinthioz \\
\hline - Kiuehinasha & Liscilenis furwio \\
\hline Thiles & 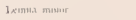 \\
\hline$\cdot \mu_{\mu 11}$ & Iytrigea lioptenct- \\
\hline - Tacbian & Clentouna tulenets \\
\hline - Stumnisutic & sporses jasmoci. \\
\hline +Nixuyuh & Deutria siclvolitiana \\
\hline - Nohbahwa & Risa banksix \\
\hline illime yut & LAhous crnonlor \\
\hline "४⿻上丨! & Lyclus $=$ entus \\
\hline - Waratalu & Actantala polygevas \\
\hline -Zakure & Punicu gatmetum \\
\hline ¡tingena & 11 ypeicuni chatienes \\
\hline ¥Harks-nanten & Nandàna damestict \\
\hline Fessen & Clcmulis flonita \\
\hline :Kinthim1 & Rlichlodenutron ohiusun \\
\hline $\operatorname{sintaght}$ & Rhoslatendmo natectutham \\
\hline "Kiritisa & Seluan lesnateclinecunit \\
\hline - N3icurebaths & 1.Altem flsuthergianaw \\
\hline -Kingaikua & Grvilyesa-parvifinat \\
\hline -Xailotukn & Diuntlus tijertias \\
\hline $\begin{array}{l}\text { "Kamaramoler } \\
\text { sluhe }\end{array}$ & Dianthas superlas \\
\hline tTcTimyus & Litian lungethofum \\
\hline
\end{tabular}

\begin{tabular}{|c|c|c|c|}
\hline $\begin{array}{l}\text { Evetsisit mritt AR } \\
\text { XAME. }\end{array}$ & JAEANEST Xisur & Prasavira: NayF. & $\begin{array}{c}\text { I Gegisit R PULAR } \\
\text { NAMr }\end{array}$ \\
\hline \multirow[t]{5}{*}{ 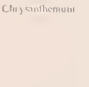 } & 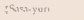 & 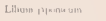 & $\mathrm{LH}_{4}$ \\
\hline & t11anhis nu, & 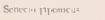 & \\
\hline & Ithenketys: & Solun eryllindicum & \\
\hline & "Kuma-z tores & Purcloeman mornam & \\
\hline & Kalked as" & Naplar panatacum & \\
\hline \multirow[t]{5}{*}{ 117ibugen } & matati. & Clejert justais. & \\
\hline & "Kodevisier & yutsex cautnoutovi- & \\
\hline & Thisaluen & 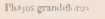 & \\
\hline & Truts-1 & seirnus ireustuk & \\
\hline & $410=0+1$ & Juncut enmosums: & \\
\hline \multirow[t]{6}{*}{ Lity } & -s,anhiskest & Sanus larustric & \\
\hline & -Kayilsarigus & Csperusims & \\
\hline & "seadiar & Meic malarset: & \\
\hline & -11.ancofiritid & Inis teviging & 113 \\
\hline & -Kw<t-הyagros & Iris whitien & WhH \\
\hline & "M!ash.cidn & Vitevintolix & \\
\hline Chemvis & - Nichaniclato & Vines ricte & \\
\hline Aralea & Thoh n.. & Carthatear lnetorion. & \\
\hline \multirow[t]{2}{*}{ Atakts: } & finserto & Fhater yprunc? & \\
\hline & thibateglata & Ixie Toregrata & $1 m$ \\
\hline \multirow[t]{5}{*}{ l.15) } & -HIthiclerilic & vertest forlada & \\
\hline & "Kisansigkn & Relnecten carnes & \\
\hline & *Karuma & Joplans reger & \\
\hline & - Crelar & Aiclsi papoiuea & \\
\hline & tholbakit & Maignolu liyponteuea & Bitgroulox \\
\hline
\end{tabular}

\section{SIXTH MONTH (TRESENT JULY).}

\begin{tabular}{|c|c|c|c|c|}
\hline In wyese Naxtf. & Fotasical Nams. & 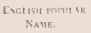 & JaFasicst Xa in & Tine vetrat XAMIP \\
\hline$\jmath K 1 L u$ & Cheyeanthemen compariuss & Chrzscuthocroum & thetaiclinkis & Diand bas clias ม⿻ \\
\hline 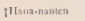 & Narulua inourtien & & - 11 isli & Trayt lsispiains \\
\hline foruats. & Flunter jugniaca & & -1Ishucholie & Sinusa fievile \\
\hline tisan & & Arclinel & tNulesulino & Dabiliue sujecibu* \\
\hline Shurabatia & Minthile julap? & & & \\
\hline
\end{tabular}

KXIT ISI TWEHAR NA QE.

Kinit of tonmine 


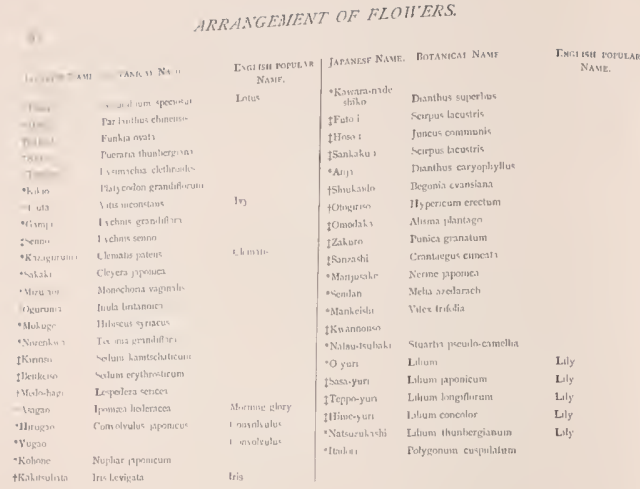

SEVENTII MONTH (PRESENT AUGUST).

\begin{tabular}{|c|c|c|c|c|c|}
\hline JAPAXFEF NAAF & BOTAMCAL NAнE & $\begin{array}{l}\text { E. otssit rorulak } \\
\text { Nastr. }\end{array}$ & Japants. Nase. & BOTANICal, NAME, & 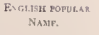 \\
\hline thillu & Chrytuntheram cokninfiom & i hry wanthemam & Itlirugan & Convolvulos jagronicus & Convolvelus \\
\hline 'Kthin & Thtyeothn granilfororuts & & 'Hag & Lespoleza bicohr & \\
\hline Itian & & Irichal & I Whash ridh & Jeggonis evansanas & \\
\hline 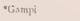 & 1.j chans grandifinas & & IKithonic & Nuphas japonicutrt & \\
\hline : Hhather & Mibiecus ynoted & & thita-i & Sciryas lacustris & \\
\hline :T:uta & Vitio monnetans & bis & flloson-1 & Jureve enmimuma & \\
\hline - Sontaichis? & Ainngähreni glaluics & & ISankaka-i & Scimps lacustric & \\
\hline - Utobletiagr & legeilexs sericen & & tolloru-int & Manchorin vasumalis & \\
\hline thasu & Nitumisura specsimunt & Lotus & thoodika & Alisma plantagn & \\
\hline Ithoruma & Inula Irratsicsez & & fOtngirios & 11 rpericum enatum & \\
\hline - Santia & Chluavthus Iracliystachys & & *Shion & Aster brlaricas: & A $\leqslant$ ler \\
\hline "Kurv & 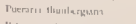 & & Keilo & Celosin argenica & \\
\hline tominingaha & I'strums ecthoss bin & & fNawa-gikis & Letuetra sesciliforia & \\
\hline t人ugao & Ifromaer bolkerseas & & Hiscokna & Jnyzatiena balanina & \\
\hline 'Jllibi & Trapa tiquanes & & Fuya & IfLiscus motabits & \\
\hline tYugan & & Convalvalus & *I. I.-getio & Amaranthus mulancliolicus & \\
\hline
\end{tabular}




\section{FLOWERS ACCORDIAG TO THEHR MONTHS.}

Jaharest Nisht. IotaktCat NamL

\begin{tabular}{|c|c|}
\hline Damiluh & Canina bullica \\
\hline tHiingr & lardanthus chinams \\
\hline - Uken & Cuncutmi loiget \\
\hline -Kicleijuse & Kuinceh in canxe \\
\hline rKuslable & Klaus semoloslata \\
\hline IKrikitsulatha & Iris leviguta \\
\hline "Turitabute & Aosnitum fincheri \\
\hline tManglowile & Lyeoris radat. \\
\hline tMarateysth & Vilex trifota \\
\hline - Dicuheses & Sxduia erytirosticluar \\
\hline
\end{tabular}

\begin{tabular}{|c|c|c|c|}
\hline \multirow[t]{5}{*}{$\begin{array}{c}\text { ENGLIsu ropulath } \\
\text { NAנr. }\end{array}$} & Jardsest DAYEu & BuIANICAL NAML & 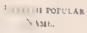 \\
\hline & fliskuchohs & Scrosa fuetita & \\
\hline & tKuanhotso & Rhonstis carnes & \\
\hline & Tkrakia-ghis & & Chuv anlenvian: \\
\hline & INoi & Alloper tienta & \\
\hline \multirow{4}{*}{ Inis. } & - Trura nualub & Citistris articutatu, & \\
\hline & tAsj & Daathous caryophylla. & \\
\hline & $\begin{array}{l}\text { tNatislitio } \\
\text { - Kamara nath. }\end{array}$ & Deanthes soperlies & 1 Aetation \\
\hline & sluiko & Draithos apertus: & \\
\hline
\end{tabular}

\section{HIGITH MONTH (IRESENT SEPTEMBER)}

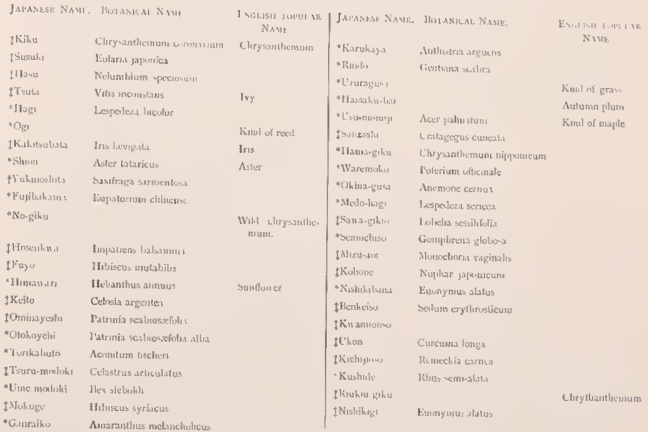


NINTH MRNTH (PIESEN T OCTOBER).

\begin{tabular}{|c|c|c|c|c|c|}
\hline "kike & 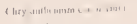 & Cliryssullictium & 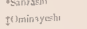 & 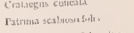 & \\
\hline - Natetios & 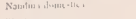 & & tolukeyesti: & 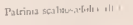 & \\
\hline Pi inatu & 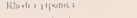 & & ‘Kocbole & & \\
\hline $\begin{array}{l}\text { :Hagt } \\
\text { tUme-taulina }\end{array}$ & 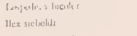 & & Sthtosuts & $\begin{array}{l}\text { Geilical icint if at ins if } \\
\text { soments }\end{array}$ & Whomezs in all freur \\
\hline - Isarsj-atialulia & Cilagerve saluul ine & & - hatratoghto & Acumtuts fialum & \\
\hline $\begin{array}{l}\text { "'is? } \\
\text { thandn }\end{array}$ & fretulis & Kiml bal sect & - R̂̀izulink & 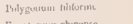 & \\
\hline -Susul. & Fulatis gVrenics & & $\begin{array}{l}\text { Yuhmoslisea } \\
\text { "Waranolie }\end{array}$ & 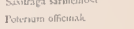 & \\
\hline t5ana-gakiu & Inbelis sosthiohis & & $\begin{array}{l}\text { Waramoles } \\
\text { fofolomaga }\end{array}$ & bejkolutd xerest & \\
\hline 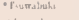 & Succio hamidion & & 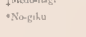 & & $\begin{array}{l}\text { Wibt clifysuithic- } \\
\text { mun }\end{array}$ \\
\hline 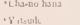 & 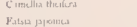 & 1. i jabit & - Lizurigusi & & Rinul of grita \\
\hline Sifsinges & 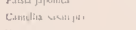 & I ans Hea & tivokiv-erho & & Clorysuabentuat \\
\hline - Fsula & Vilas anp isstais & (i) & Kobsure & 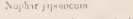 & \\
\hline $\begin{array}{l}\text { Prista } \\
\text { - thision }\end{array}$ & 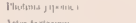 & & thisto wevis & 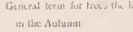 & ice of 14 bicl rottlen \\
\hline *Kithiguhila & $\begin{array}{l}\text { Istor tartasusus } \\
\text { Iriv levisits }\end{array}$ & then & t Kixwagenesow & Trass of the sillon kuml & \\
\hline I Karuliays & Andisiman aryatio & & Nobthagl & Euniyisus thatus & \\
\hline fllan:13 ty the & Clirgosathonam mprosacs & 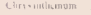 & & & \\
\hline
\end{tabular}

TLNTH MONTI (RRLSENT NOVEMBZR).

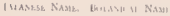
Sean yiky

sulsers Nartisos tartita

than-giko

GS surasti

* Cla-no-hama

- Hausa

$\$ N_{\text {auler }}$

-Oinoti.

- Neka-yaiagy

STsunaluaks.

Kochishy

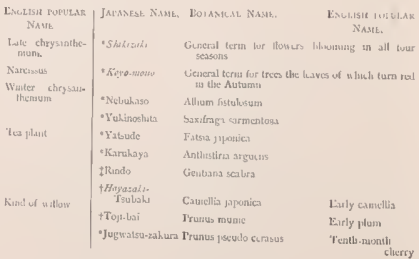




\section{FLEVINTII MONTII (TRESENT DECEMBER).}

\begin{tabular}{|c|c|c|c|c|c|}
\hline IAPASER NAME: & Retamicat Navi. & $\begin{array}{l}\text { Ixaish finger in } \\
\text { Nisur }\end{array}$ & JAEAsuc Nabr. & Ireranient Nevil & $\begin{array}{c}\text { Extisit } \\
\text { RAM }\end{array}$ \\
\hline - Kan guku & & $\begin{array}{l}\text { Woter ebrysan: } \\
\text { themum }\end{array}$ & - Aisionensuso & $\begin{array}{l}\text { Gonerat term for tree- } \\
\text { in Alue Autumn }\end{array}$ & 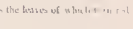 \\
\hline - Suigen & Nitcrestus tiesita & Sumercate & thesbuke & & \\
\hline -Ninteis & Nunitun dinmesties & & †Kan-1notus & Promsa minusa & 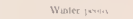 \\
\hline Strinow & Llindes jy wrict & & I Gogankwa & Cincellsa sssiogas & Convilis \\
\hline INcho-) anagi & Sain leachy altishy" & & Fralsuele & 1-at?a juyonics. & \\
\hline f rojel-mat & Trumes meite & Bucly flom & ;Tsulah: & 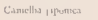 & Camallin \\
\hline t)ugastsu xาhurs & 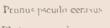 & $\begin{array}{l}\text { Testh unisth clicry } \\
\text { che }\end{array}$ & -sindrads & $\begin{array}{l}\text { Gevisral lerm fos th } \\
\text { scosctis }\end{array}$ & tonery litwomigg in sit hour \\
\hline 10ะล & 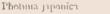 & & & & \\
\hline
\end{tabular}

TWELTH MONTII (PRESENT JANUARK).

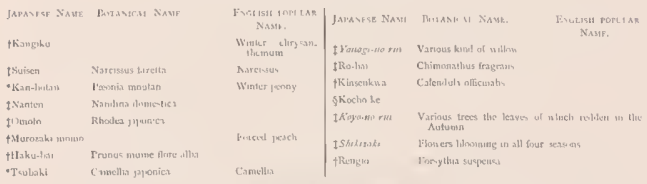

Considerations of grood or exil luck enter largely into the choice of flowers, especi ally when emplayed as decorations for occasions of rejoicing; and there are cortain Howering plants and trees reputed to possess poisonous properties in their ronts, stems. leaves, or blossons, which are objected to at any time, their employment leing consilered unlucky and ominous. The following is a list of the principal of such poisonous flowers :-

\section{ONINOCS FLOWERS.}

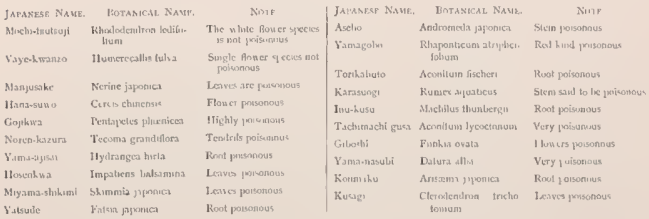


In addition to the last named, all fowers having a powerful odour are censideced unsuitable for placing before gutests.

Among the fowers peculiar to the different months previously classified, some are considered specially appropriate for displaying upon fete days whilst others, though allowet at ordinary times, are interdicted for such important oxcasions.

As most of these flowers are to be found enumerated in the complete talses aready given, the following chasification is abbreviawd, mercly giving the Japanese names and the corresponding name in English, Lotanical names being printed only where nu popular equivalent exists. It may be observed that this list includes in all twenty. four species of plants and trees, of, if different species of the same getera be classent togrether, the number of specially honoured Howers becomes reduced to filteen:-

\section{FLOWERS SUITABLE FOR FELICITOUS OCCASIONS.}

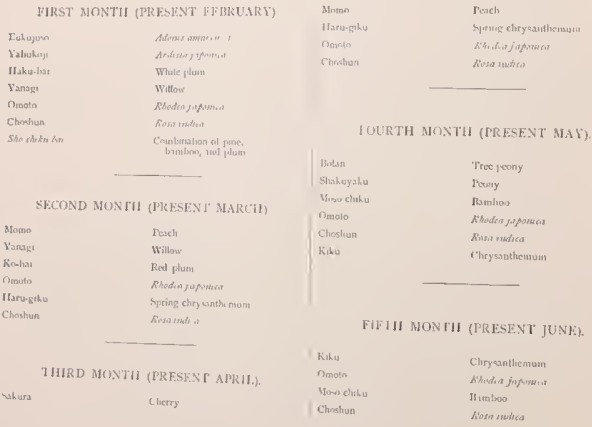




\section{SIXTH MONTH (PRESENT JULY).}

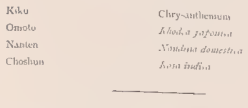

SEVENTII MONTII (PRESENT AUGUST)

\begin{tabular}{|c|c|}
\hline 1., te & Clerysanthemesn \\
\hline Chisate & 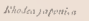 \\
\hline Cherlius: & Litrinutises \\
\hline Nantear & 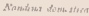 \\
\hline
\end{tabular}

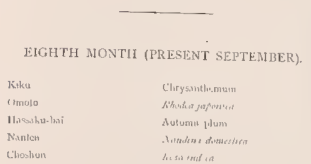

TENTII MOSTH (PRESENT NOVENGER).

\begin{tabular}{|c|c|}
\hline Langites & Clirysinthemun \\
\hline Sulctal. & Nivisus \\
\hline Umint, & 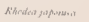 \\
\hline Natites & 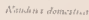 \\
\hline Clinstasia & Noson implat \\
\hline Yaitogid & Wulleni \\
\hline
\end{tabular}

TLFVENTI MUNTI (1RESENT DECHMIBER)

masea

Kins-gike

Orante

Yamg!

Naritia

Toyr-bert

Cled bur

Yaye-toulahit

Niruzts

Winter eling suthomsus

Rheifo jopatain

Willow:

Nemadians down ration

Eitly |เึ็น

Aitus uakn

Chublie comellit

TWELHTH MONTH (TRISENT JANUARV')

The following list of llowers, the use of which is prohibited for special occasions of ceremony or congratulation, is arranged without regard to the months to which they belong. The employment of such flowers is deprecated at any season, without reference to any particular month, unless no other flowers can possibly be obtained. The rcasons for their rejection are not always very clearly defined; sometines the objection is to the form, sometimes to the colour, occasionally to some supposed poisonous property, and often 
to $x$ shat $=4$ little whe thas traditional superstition or caprice. It is not surprisings.

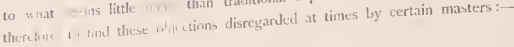

FLOWERS PROHIBITED FOR FELICITOUS OCCASIONS.

\begin{tabular}{|c|c|c|c|}
\hline & & Nazlii & Artur monericasst \\
\hline $\ln _{10}$ & 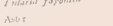 & Кенго & 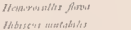 \\
\hline 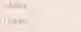 & 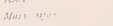 & Tuyo & $\begin{array}{l}\text { Whetecte wrathalitis } \\
\text { Lotus }\end{array}$ \\
\hline 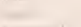 & Arotidiat witister & Rienkfe & 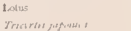 \\
\hline+4 & 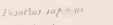 & Ifaletongt-1500 & 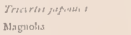 \\
\hline 1 & Kinil of net: & Muluterin & 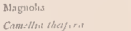 \\
\hline $\operatorname{lin} 1$ & 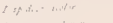 & Chomi Gitia: & 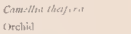 \\
\hline 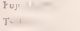 & 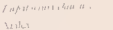 & Rum & 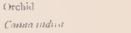 \\
\hline$T=1$ & 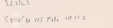 & $\begin{array}{l}\text { [15rimidu } \\
\text { Respur }\end{array}$ & 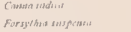 \\
\hline Mis +1 C. was & 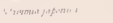 & Yosily & 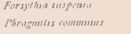 \\
\hline Мика: & 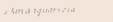 & A tils & 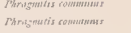 \\
\hline Kenth & - Junese Ji umilna' & Rindo & 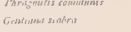 \\
\hline liet & $147<0.9$ & Am>suly & 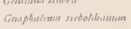 \\
\hline Dins gets ... & Lurgetiat I. h & 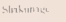 & Rhodorienilena \\
\hline 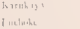 & $A+2+n+1, \quad n=n$ & Koctinnsatu & 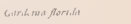 \\
\hline Fintinate & Hiflute tot? & A. & Iprowert hindentsin \\
\hline $\begin{array}{l}\text { 17. Lalsan! } \\
\text { Hital 4yithe }\end{array}$ & 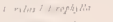 & Giturlu & Fwenkuz, whitho \\
\hline 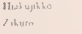 & 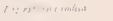 & HIज & Rardinditer anknsus \\
\hline 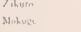 & 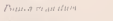 & Lnot-1umblat & Mex stoboldt \\
\hline Masunazhe & 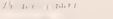 & 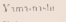 & Swathe lepatiat \\
\hline Ifincitihn $=$ & 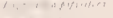 & Kahos & Naphar stenti wew \\
\hline Konw & 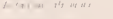 & Junk he & Thesterethtris \\
\hline Kistor & (3) $\cdots,+1$ & Velsude & Firstit givaraca \\
\hline & 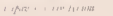 & Afotis & 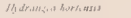 \\
\hline
\end{tabular}

Ranking hishest in the ibove list of felicitous flowers, the following seven are considered as par chelluns: those for ceremonies and congratulatory occasions :-

The Kiku, or Chrysanthemum, to which is given the fancy name Choju-so, meaning Lumb-lasting Flant, on account of its growing through all the four seasons.

The Situsen. or Nafcissus, ealled by the fancy name of Invo-so, of Plan of the Taw

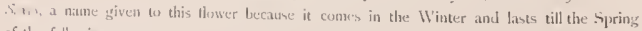
of the following year. 
The Mimijz, or Maple, fancifully called Dokngo-so or Mron-dispelling Plaur, because there is a popular superstition that it absorls all poison and infection from the air.

The Saztuma, or Cherry, regarded in Japan as the king of tlowers.

The Bolan, or Tree Peony, fancifully named Frokz-gusit, meaning Ftant of Wialth and High Rank. The peony is saikl to be the queen of flowers in China.

The Omotis, or Rlioden japonica, much honoured becausc, unaffected by heat or cold, its leal remains strong and green throughout the year.

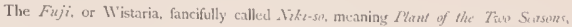
because, appearing between the third and fourth months, it butongs both to Spring and Summer. Though much honoured and used for felicitous occasions, the wistaria must not be employed at weddings on account of its purpte lue, this being associated with mourning.

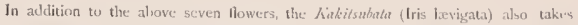
high rank, but on account of its purple colour, like the wistaria, it is prohilited for weddling ceremonies.

Hitherto attention has been directed to the principal flowering plants and trees of the country, and to the degree of esteem in which they are individully regarded. especi. ally with reference to particular months. Many foral compositions consist of combinations of two or more different kinds of growth. The manner in which different species are combined is best explained when the whole theory of the Jipanese methouls of arrangement is discussed in a later chapter. But, apart from the manner of grouping them, there are certain prejudices in favour of and against different combinations of material which require mention in the present context. The following is a list of a few of the suitable and unsuitable combinations of tlowers :-

\section{APPROPILIATE COMBINATIONS}

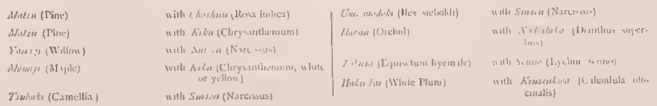




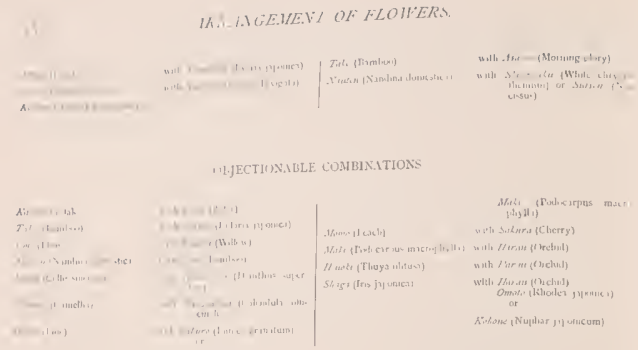

All of the uhive conlunatians, both good and bad, are of trees or plants which in in bloom during the same nonth. They are, therefore, combinations which are practicable without violating the rules as to seasonableness. Those which are classified as objection. able are so considereil, therefore, for reasons other than that of seasonableness, Sometimes the objection is lased upon two clese a resemblance in form or colour $\mathrm{i}$ in other cases, similarity of species, or of locality of production, leading to redundancy of expression in the: composition, is the deterring cause. The peach and the cherry, for cxample, being both flowering trees and somewhat similar in claracter, are not considered suitable in combination. 


\section{HISTORY AND THEORY}

EFORE proceeding further with an explanation of the Flara] Art, it may be of some interest to enquire into the erigin of a calt 50 curiemaly unlike, in its methods. any other hitherto followed in Europe, Japancse histoxians daim for it an Indian and religious origin. The doctrines of Buddira, deprecating as they did the wanten sacrifice of aninal life, are said to have suggested the gathering of fiowers liable to rapid destruction in a tropical climate, and prolonging their vitality by careful prcservation. The survival of such a theory would scem to show that some form of the art was first introduced into this country with the adoption of the Buddhist faith, both as a part of its ritual-flowers being placed before the Buldhist spirits, - and also to provide a pious pastime for the priests, The religion of Sakya MIuni, as is well known, reached Japan through (hina in the sixtl century, and certain Chinese priests are mentioned as the first tcachurs of the art of arranging flowers in Japan. It also appears that the carliest native jractitionurs in this country were fumous priests, amongst whom Shotoku Taishi, son of the Emperor Yomet, and Mteilkei Shonin, are particularly mentiunecl.

These prinitive flower compositions were, however, of a more accidental, and, at the same time, of a more extravagant character than those of the art as it became afterwards modified and developed. They partock more of the nature of a Vestern bunch or nosegay, being crowted in arrangement and miscellaneous in substance; they also lacked the severe conventionality of later methocks. The style of cumpusition adopted still survives under the name of Ruktust, meaning Erect Flewer Arrangement, and is uset for flowers placed as religious ornaments or offerings bufore shrines and tombs and as a votive decoration at marriage ceremonics. An approach to symunetry was a governing fiature in the most clabrrate of such compositions. Branches of blossoming trees or foliage were eniploged, to form a vertical central mass: and other flowers or bunclies of foliage were clispased on either side in balaneings groups. The idea of imparting graceful and har. monious curves to the differcnt lines of the composition was as yet only partially deve- 
loped. Unlike the later and nore refined flower arrangements, this early sty-le warenarkable fore the nixture of a variety of different material, as many as twelve or more species of plants and trees beins emplaycel in one design. The chict lines of a conposition. gencrally seven in number, were formed of branches of different grownth. some of which were in full leaf or flower and others purposely light and sparse in character. Large leaves of other plants were used at the base or connection of these 1.rjous brancies to hide their bareness, and carcful attention was given to the bends and curves of these leaves so as to reveal their front and back surfaces in a well balancel contrast. The shape and disposal of the hollows or openings in a floral design received as much attention as the principal lines. Terms such as,-"valley," "grotto," and "perspective distance," were applied to these openings, the fancy that a matural landscape was represented being always kept up, Even in this comparatively ancient development of the art the proportion which the floral conposition held to the vessel which contained it was fixed by rule, a practice which was followed in all later arangements.

A special branch of the Riktrita style, which approached to a kind of miniature sudening, was applied to the ornamental use of thick stubs and branches of trees and water plants arranged in broad shallow vessels with an admixture of small rocks and stones, The intention of pourtraying and sugkesting landscape, which to the Japanese is present in every class of floral composition, is, in this particular styic, patent to every olserver. This hisd of arrangement, in a somewhat degenerate form, may be frequendly seen at the present day in Aloral decorations for large public roms, old lichen-coveral branches of pine, plum, or maplic: trees being the favourite material.

The Rikitur style possessed numerous rules and an elaborate nomenclature applied to the different memhers of a composition. Plates XI. to XXI. inclusive illustrate the firktave style of flower arrangement. The later and more popular styles, which it is the principal object of this work to expound, adopted, to some extent, similar terms and theories in a simplificd form.

The more modern developnent of the filoral Art was simultancous with a great fancy for ornamental vases of variuus kinds, which sprang up uncler the patronage of the famous Regrent Yoshimasa, at Higaslij-yama. The impetus given to the manufacture of choice vessels by this Prince, chiefly owing to his inauguration of the Tea Ceremonics and other polite accomplishnents, was accompanied by an austere refinement in the methods of arranging Howers. It was mainly with the object of adaptation to the Tea Ceremonial that the first modifications in the Flower Art took place, and the chicf reformers were the 


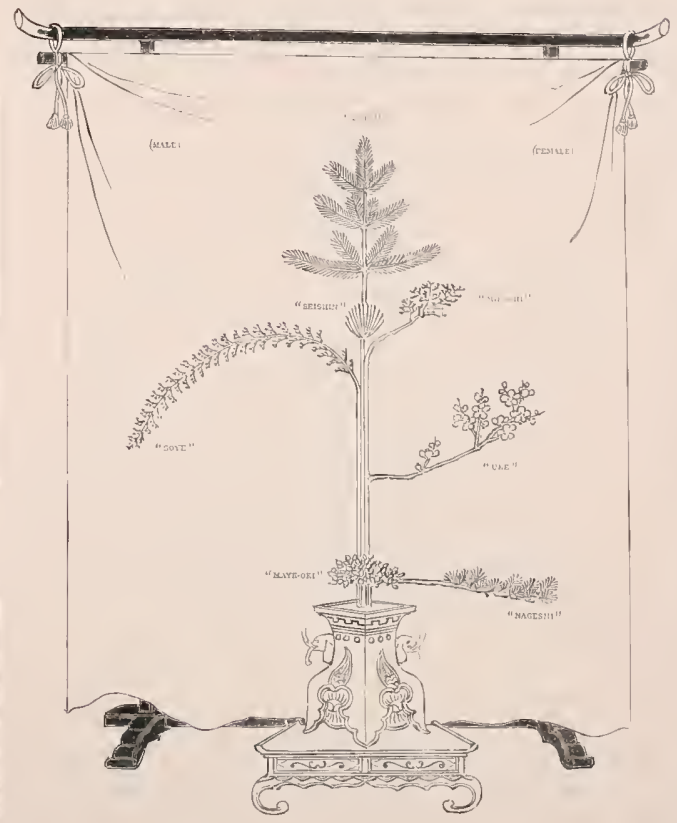



PLATE XII.

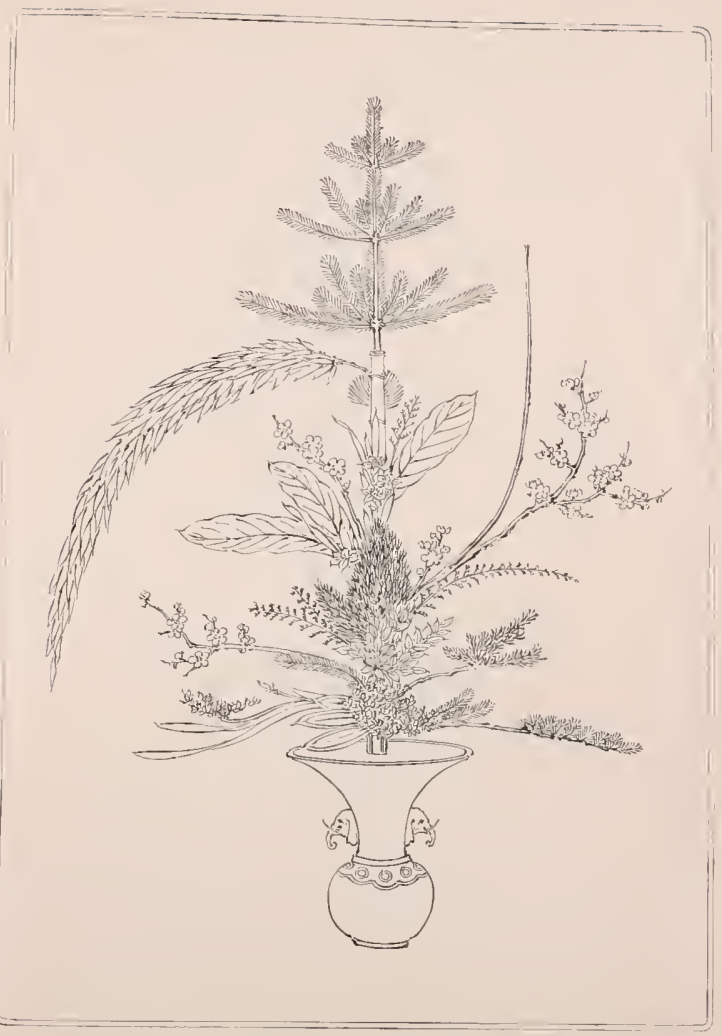

STRAIGHT RIKRHYI ARRANGEMENT, SHIN STYLE. 

PLATE XIII.

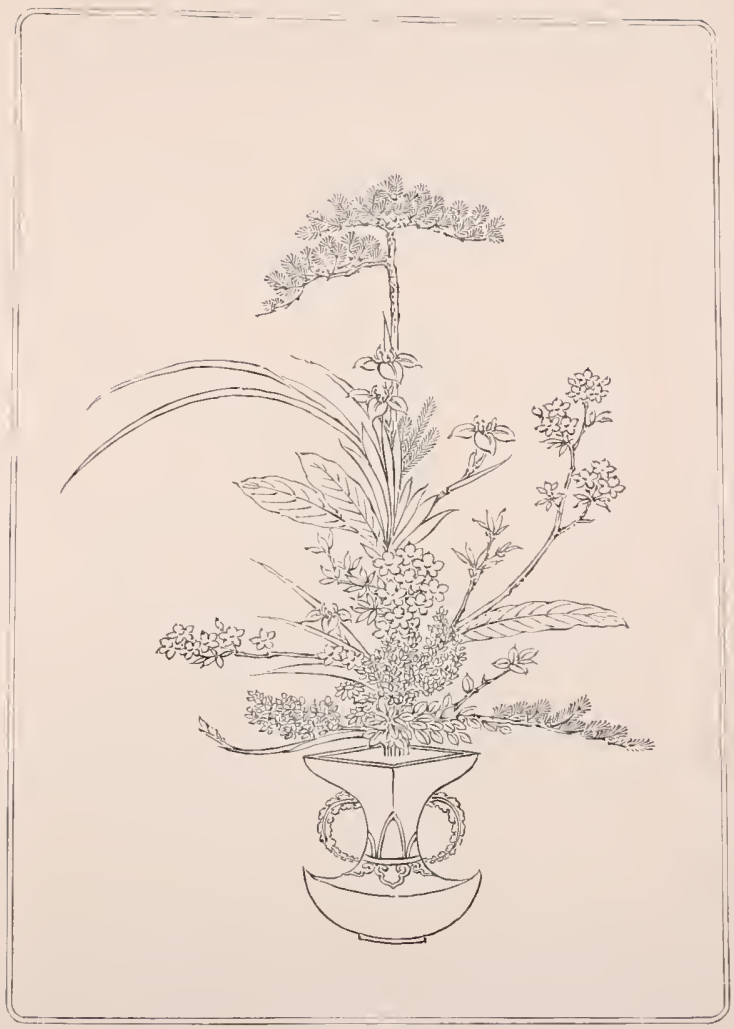

STRAIGIIT RIKKHA ARRANGEMENT, GIO STYIF. 

I'T.ATE NIV.

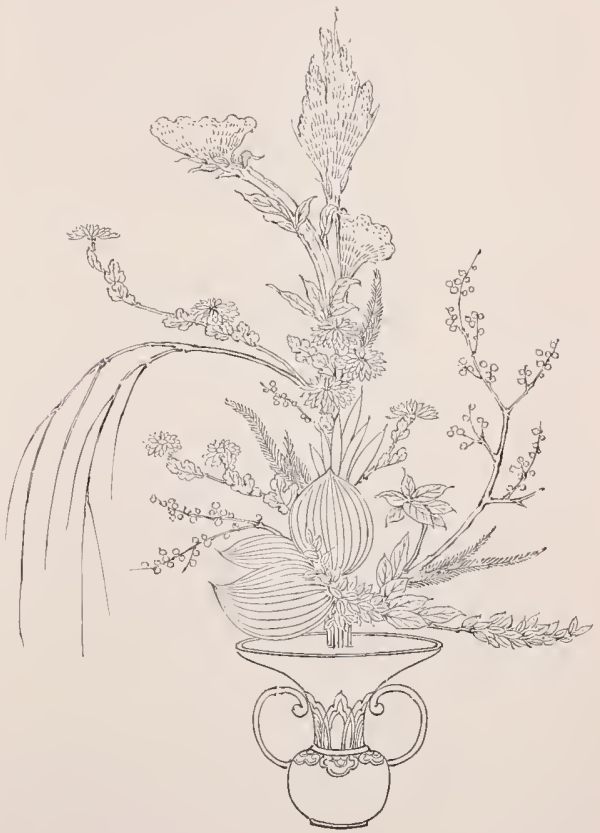

STRAIGUT RIK'A'IA ARRANGEMENT, SO STYLE, 



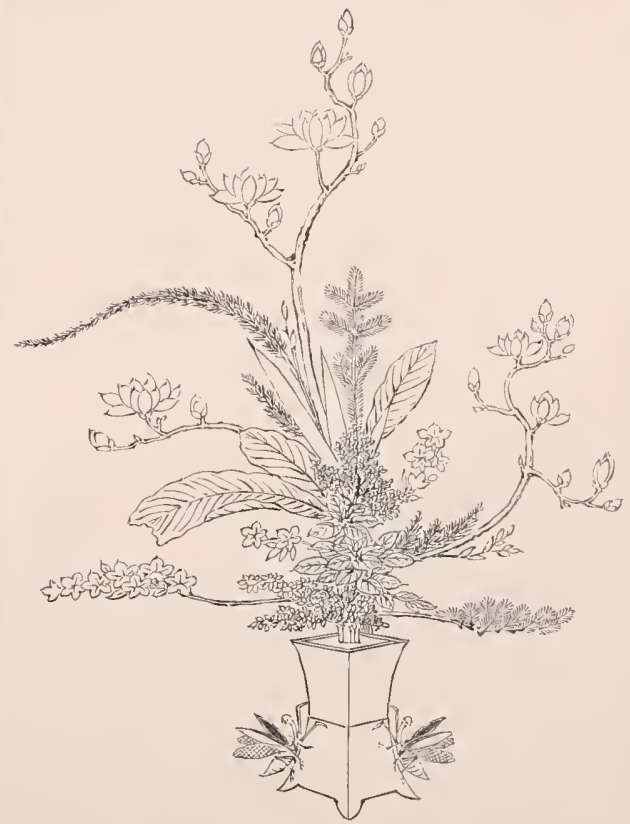

BENT RIKKWI ARRANGEMENT; SIIN STYLE 



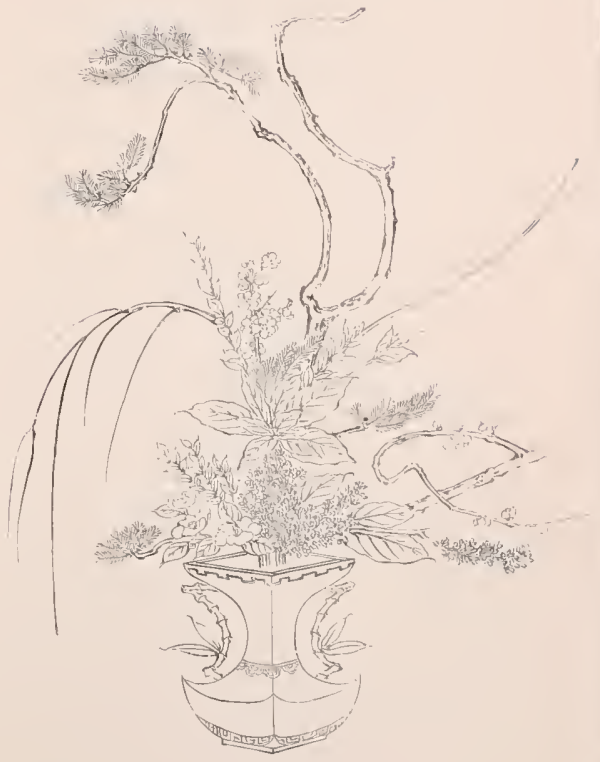

BIWT RJKHII ARRANGIMENT, GIO STILI: 

PLATE XVII.

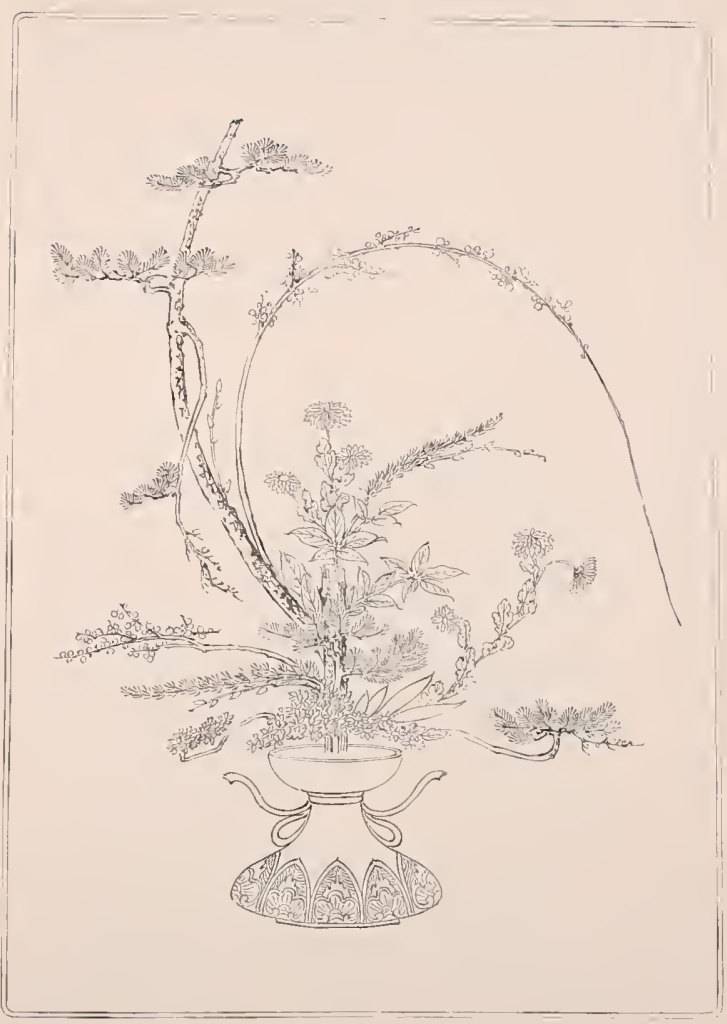

BENT RIKKIH. ARRANGFMENT, SO STYLE. 



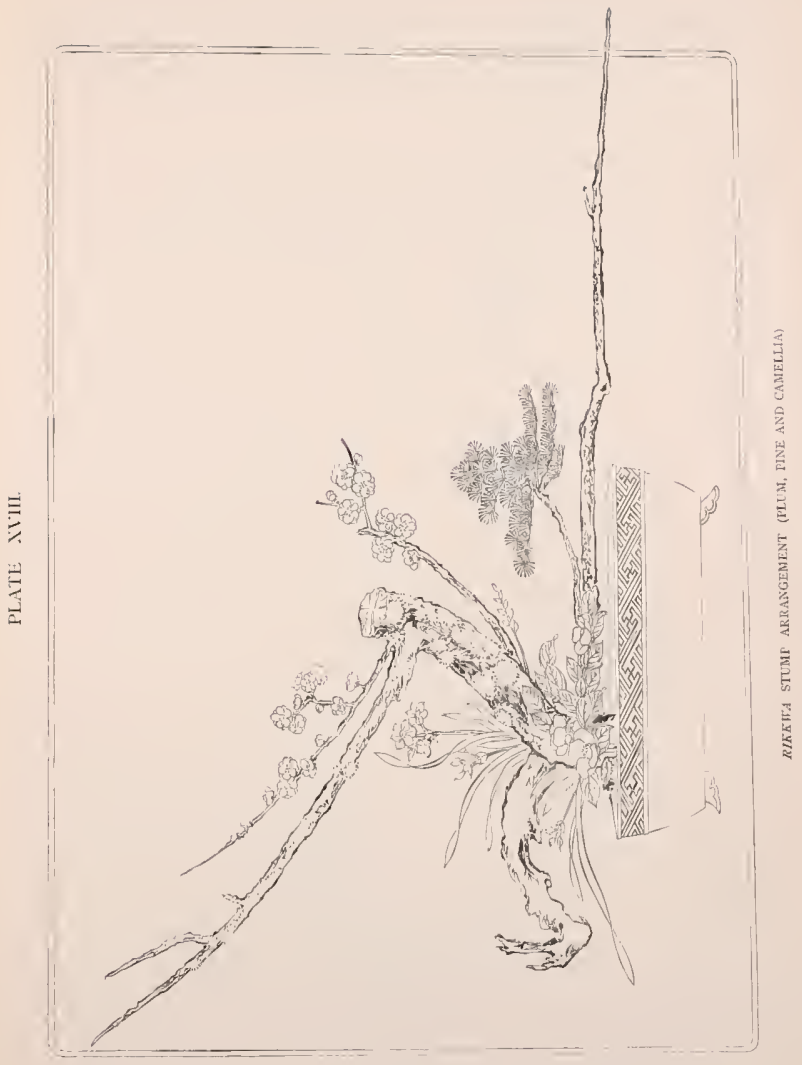





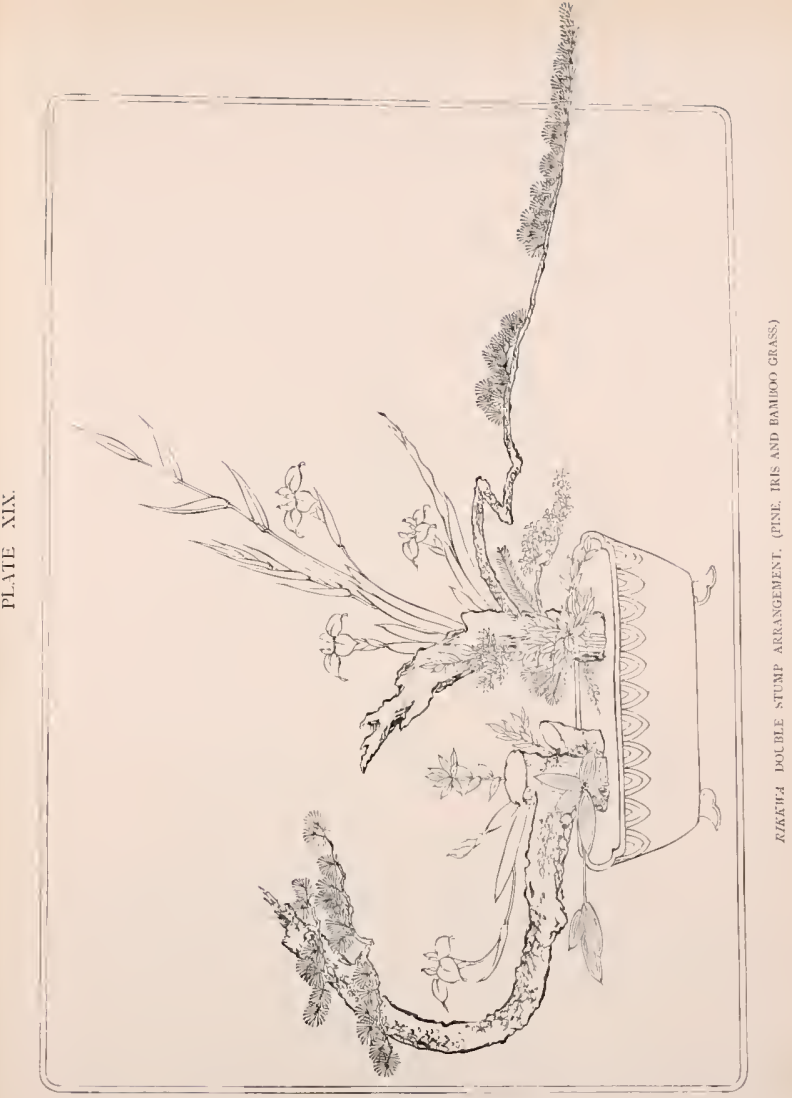




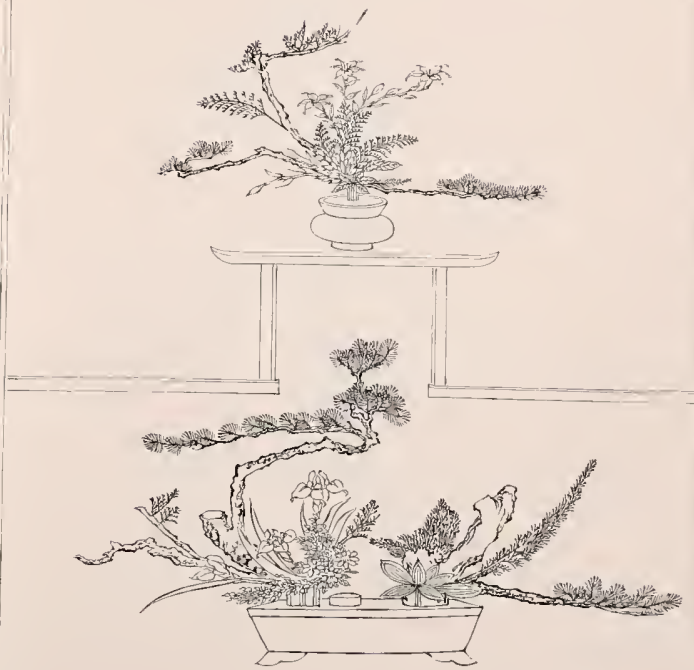

RIKKHA COMBINED STLMP ARRANGEMENT FOR SHELVES 

PI.AT1: XXI.

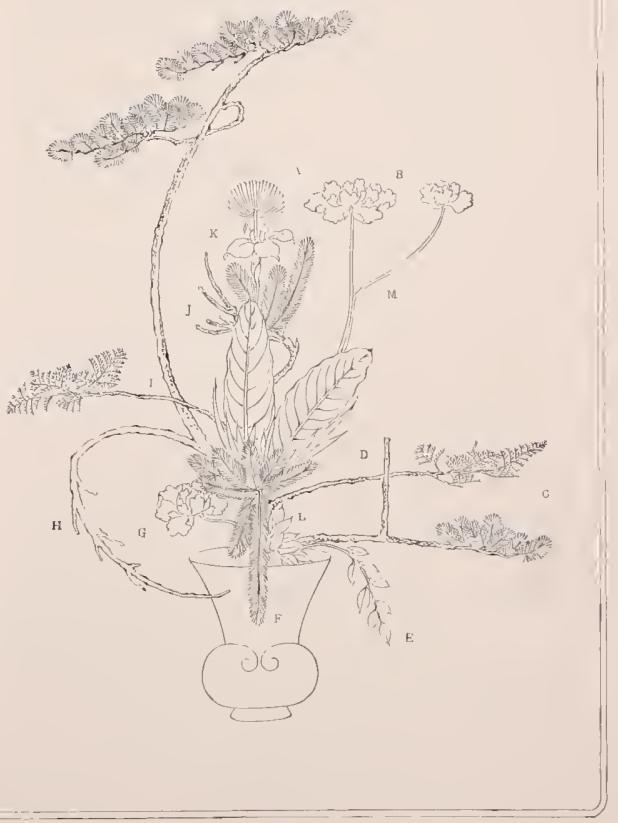

RJKIIA DHGRAM OF ERROKS. 

Chajin, or Professors of Tea, Sen no Rikiu, Senke, Sekishiu, and Enshiu, who were four of the principal flower designers of the Ashikaga and subsequent perinds, were at the same time fanous professors of the Tea Ceremonial. But the affected simplicity which ruled tise Tea Room did not give full scope for the exercise of great elaboration in flower compositions, and other less austere forms of arrangement were developed, suited to the larger chambers of the nolility: Lut based upon the principles which bad thus been inaugurated. A distinctive character and special proportion are given to flower compositions, as thus finally classified, according to the particular class of chamber which they atorn or the rank of the person in whose residence they are usecl.

The arranging of flowers has always been regarded in Japan as an nccupation befitting learned men and Literati, and though the ladies of the aristncracy have practised it. tngcther with other polite arts, it is by no means considered as an effeninate accomplistrnent. Among its most enthusiastic followers appear the names of noted priests, phifosophers, and even famous statesmen who have retired from public life.

Mixed "p with the theory of the art, and imparting to it at frost sight an air of quaintness and mystery; is a considerable amount of Cluinese plilosophy; togrether with many traditional superstitions. Ideas of good and evil luck control both the selection of material and the manner of aranging flowers for special occasions. Various virtnes are attributed to the professors of the art, who are considered as belonging to a sort of aristocracy of talent, enjoying privileges of rank and precedence in society to which they are not by hirth entitled. A religious spirit, self denial, gentleness, and forgetfulness of cares, are some of the excellencies said to follow the hahitual practice of the art of arrangement of flowers. Philosophical classifications are resorted to for the purpose of elistinguishing the clifierent parts of floral designs. Thus Earth, Heaven, and Mankind are names employed in some styles to indicate the parts of a tri-lineal flower arrangement; Earth, Fire, Water. Aletal, and Wood heing used in the same way to designate the mumbers of a fve-lined design. Other Schools apply, in a similar manner, the names of abstract ideas, such as the five orders of Japanese versification, or the virtues of the buman heart. The different methods of nomenclature are numerous, and would appear to have been adopted hy the rival Schools principally with the object of imparting an appearance of originality and mystery to their own particular versions of what is practically one and the same art.

Again, the male and female principles so often referred to in Confucian plailosophy are constantly applied to distinguish contrasting forms, surfaces, of colours in composition. It has ever been a favotrite fancy of the Japanese to apply distinctions of sex to inanimate 
nature. In natural scencry: and landscape-gardening, it is customary to discriminate between wak and female cascades, mule and fomale plants and trees, and mate and fowale rocks and stones. The distinction is not so much one of individual and separate quality as of fontus placed in combination or contrast, and regarded as male or fimale in respect of one anoticer. Thus the main torrent of a waterfall is considered masculine, and the lower fall in provimity fomitume. In like nanner, rocks used in gardening have no distinguishings sex. voless they are used in pairs or groups. In the case of two stones of different character places side by side, the one of holder and more vigorous shape will be called the mate, and the other the female stone. Curious as such fancies may seem. they are of considerable value when applied in the arts of design, their ohservance helping to prochese that harmony of well inanced contrasts which should pervale all artistic compositions, Nor are such ideas, indecd, quite foreign to certain branches of Western art. In architecture, for example, it is common to attribute male and fomale clarneteristics to the different onters of classic architecture.

In the Floral Art the idea of sex is applied to cuntrasting forms, long and short, large and small, angular and round, as well as to slifferent kinds of growth, and to varions colnurs in combinations. When a flowering tree is used in combination with a plant, the tice is considered as malc, and the plant as fimal: Widh blossoms, buds are regarded as fomalc; full Powers as malc, and over-blown Howers again are classed as fomalc; the time of full vigone recives the make, and the periods of weakness the femate character. A similar fancy is applicil to the different surfaces of leaves and to the different colours of Howers. Among coloturs, red, purple, pink, and variegated hues are mate; and bluc, yellow, and white are fimale: The front surface of a leaf is malc, and its minder surface is fimale. W'ith Howers, the different forms of hud and full blossom, and in the case of leaves, the two surfaces, lend ticniselves easily to such distinctions; but berries seem to have defeated attempts at sexual classification, until Enshitu, one of the mansters of the Flower Art, after observing the bulbul bird pecking at the fruit of a tree, devised the method of marking certain of the berries in a floral composition as if pecked by birds, and thus creating a dual character, that of square and round. The mate and female principles are also applied to the different directions of the stems of a composition, the right hand side and from of the design being regarded as malc, and the left hand and further sicle as female. A strm on the left sicte of a composition turned to the front and back to the right is said to have: male character, while to one on the riglu bent back to the left is attributed female character. 
Besich sex, the difforent colours in flowers or leaves are sail to have an order of r.tuk. This idea of rank is applied principally to difierent colours of the same flowers. With most plants the white: flower takes highest rank, but there are exceptions to this rule. The yellow clirysanthemum takes prececlence of those of any other colour: With peach blossoms, the pale pink colour ranks tirst, though there exists a rich red specinen of great beauty and rarity: In the case of other flowers the colours raking the lijglest rark are with inses, purple, with camellias, sed: with wistarias, lavender: with poesties, resl. with valerians, yellow; with convolvuli, dark blue, with kerrias, yellow; with kikios, light purple: and with lespedeats, pink.

As before mentioned, a number of different Schools of Flower Arrangement squany up after the inputus given to culture by the great art-patron. Yoshinasa. The principal styles of composition practised by these different Sclook are known as the Ki-Rill, $/$ kermot,

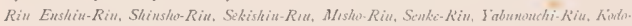
Rin, and Seizon-Rm. The masters of any of these Flower tichools would resent as ignorant the idea of confounding their theories with those of a rival style. Each School has its orm special terms, philosophical mysteries, and secret dugrees, but to entur closcly into these peculiarities would be almost like discussing some of the more trivial differences between sects in the same religion. The main principles of the art are the same throughout, and the Horal designs produced, regarded as works of art, are as similar as any desings can possibly be. The compositions of the Rikkata and Jkerwo styles are the only ones which jresent a distinct character casily recognisable from those of other styles. In the present work the Fnshizt style has been chictly followed, this lxing the most elabotate and one of the most popular of the more modern styles, but numerous illustrations liave been taken from compositions of other Schools.

The Eushen style was originated by a retainer of the Shogun Iycyasu, called Kobori Totomi no Kami. He was a distinguished professor of the Tea Ceremonial, and became teacher of this accomplishment to the Shogun's heir, Tyemitst. Compared with some of the other styles, that of Enshiu is characterized by a greater elegree of artificiality or artistic affectation, and this makes it specially adapted for the purposes of analysis for and a thorough explanation of the principles of the art. 



\section{LINEAL DISTRIBUTION}

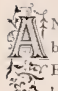

$\mathrm{N}$ analysis of Japancse flower arrangements shows that the lines or directions taken by the different stems or branches form the basis of all compositions. While European floral decorations are merely combinations of masses of colour, in which blossoms and leaves alone play a part, those of Japan are synthetic designs in line, in which every indivitual stem, lower, and leaf stands out distinctly sillouetted. The treatment employed may be likened somewhat to the methods of distributing carved foliage in archicctural pants as followed during the midlle angs in Europk:

The surface of the water in which the flowers are placed is technicalis emostitered to be the soil from which the floral growth springs; and the designer must lece contey the impression of stability and strength. Honever goud the upper lines of the composition may be, a weak springing at the base deprives then of life and vigour: for it must be remembered that not flowers alone are displayed, but floral growth and vitality are $w$ be expressed in the designs. The direction of the stems at starting need not bu strictly vertical, but, if curved, the curves employed should be strong ones, and all weak bends and angles should be avoided. As a composition consists of several main lines, it follows that there will be several lines of springing, or origin. In songe cases, the springing lines are all united from below the surbere of the water to a point some distance above. after which they separate in tamgental curves in different dircetions; in ollyer cases, each stem-line is kept distinct throughout, being separated from the others inumediately from the point of origin.

In the distribution of the principal lines of the composition from the point of their separation. the artist studjously avoids an equal-sidet or symmetrical arrangenent, but he obtains a balance of a more subtle kind, which is at the same time productive of at pleasing variety of form, Balance and harmony witbout repetition is a governing principle in this as in other japanese arts, The lines of each stem, or, in cases where numcrous slender stems are combined, the central lines of each group of stems, receive first attention, 
The trijt arrang it $-b y$ which is meant that governed by three prevailing lines, may be takels as the orignal model for all arrangements (sece Plate XXII Ar).

The three lines of such compositions may with sufficient fidelity to the more quaint native nomenclature, be called. Primcipal, Secondary, and Tirliory. The Primipal. is its nume implies, is the central and longrest line of the design, and this is made to forns a double curre with the upper and lower extrensities nearly vertical and in a continuous line, the general shape thus assumed being that of an archer's bow. The Siroudary should be about half and the Fertury about one quarter, of the length of the Arincipul, supposingt all to be straishtened out: and these tiva lines are arranged on different sides of the Premespal in graceful clouble curves of varied character. As a general rule, the Secondary has a more vertical, and the Tirtary a more lateral tendency i the former being on the

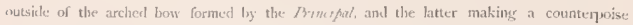
on its hollow site. Aceorcling as the hollow of the Principal laces riglut or left the arrangement is called a right or left composition. By clanging the elirection and giving a different character to the curves of these three lines a great number of designs are produced, Some of those are shown in Plate XXII $u$. the more violent curves being favoured by the Enshin School.

To produce a five-lined arrangement, two alditional lines are introduced between the three jorviusly maned. The one placed hetween the Primofal and the Scroutary is callect the Support, and that between the Brimipal and Tertrary is called the Sizb-princifal. The Suppw\%, in its length and value, approache's more to the Sctomdery than to the

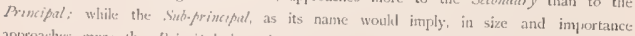
approaches more the Principal than the Fortiery. In this way, it may be observed, a proper lineal balanee and harnony is ubtainecl. For supposing fagures are used to indicate relative size and importance, and supposing the three dement.ury lines to be valued 42 , and i. according tor their respective lengtlis and degrees of imprortance, then, to preserve a proper balance with the addition of two extra lines, that introduced between + and 1 should be longer and nearer in value to 4 than that between 4 and 2 , which should approxinate more the dimensions and character of 2 . These additional lines, besides laving difierent interntediary lengths, have special curves given to them, and are arrangred so as best tu fill the intervals, with due regard to variety and balance. In the seven-lined the Trusk-line. Their lengths are about internediary betweut the Side-line and the other

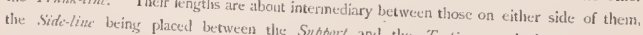
between the Sub-primeifal and the Sccoutary. The different nembers of the above 


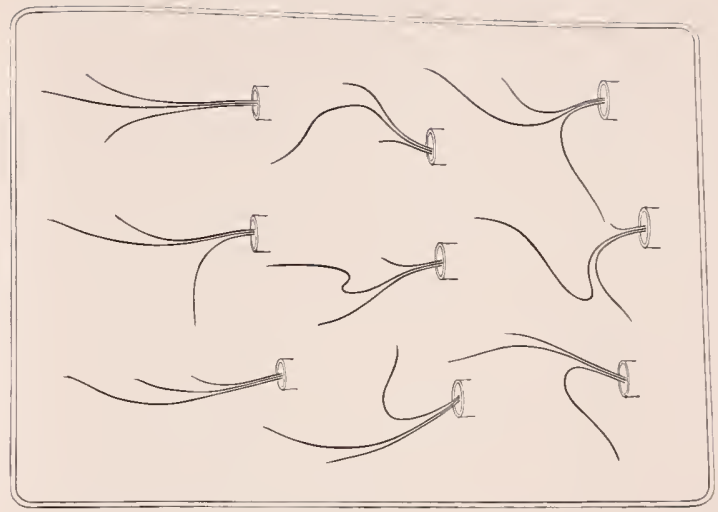

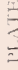

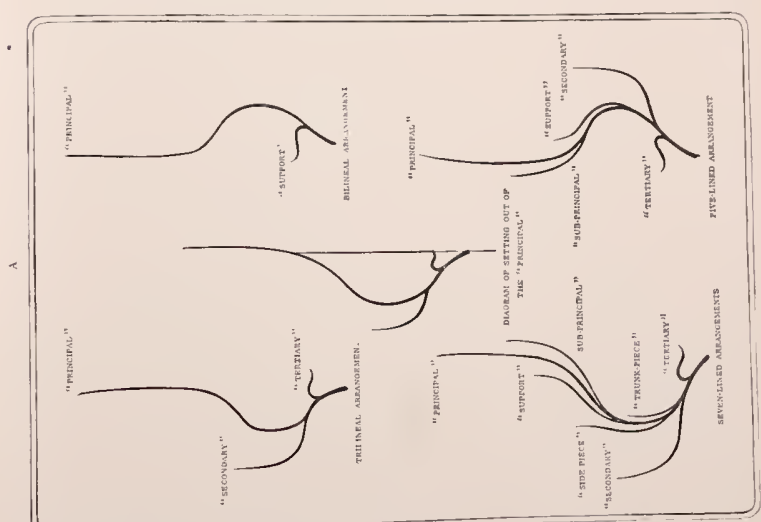

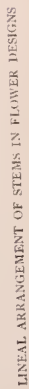



bestowed on them by the different Schools. For the triple styl. such classifications as

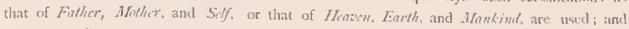
for the live-lined style the tirms Centre. North, Serult, Eect, Hist; or Earth, Fire, Iriter. Metal, Hood: and sometimes Hillene, Red, Black. HYite, and Blue are employed. The Fushir School apply the following curious ideas to the different lines in an arrangement.

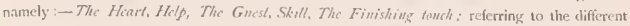
sentiments which should inspire the designer in his treatment of the different lines. This School maintains that there are three secrets of arranging flowers. First, the art of giving expression and feeling to compositions: Second, the art of showing the charicter of the materials, whether plants or trees: Third, the art of keeping in mind the season and occasion of the arrangement by avoiding incongruous combinations.

The general form of the above groups of three, five, and seven lines depends mainly upon the amount of curvature given to the Primipal or centre-most line. In the simpler and less affected styles, the bow-like curvature of the frincifal is slight and strong, but in the more exaggerated compositions this groverning line is holdly bent side ways from a point a few inches ahove the springing. and turned in a quick curve back again so as to bring the upper extremity vertically above the base, and to preserve the centre of gravity of the whole. The general form thus imparted is, as mentioned above, somewhat like that of a strung bow. From such a shape the transition is not great to that producted in the atc of a bow by drawing its string, a more violent lateral curvature with less vertical height being Iro- $^{-}$ duced. This latter character is given to the Brincipal chicfly when used in positions where too much height would mect with actual or optical obstructions and be detrimental to a good effect.

Such treatment is often followed in floral arrangements placed below a shelf or in front of a hanging picture which must not be hidden. This modification in the character of the Primciful necessitates corresponding changes in the direction and curvature of the other lines of the composition.

Up to the present, slight or violent as may

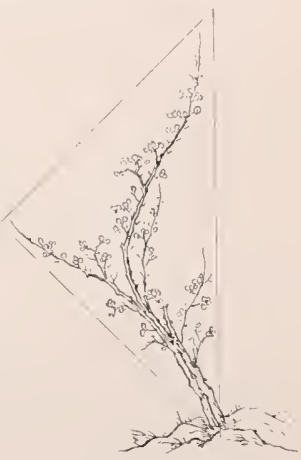

Fir, $t$. 
be the bow -like arvature imparted to the Principal, it has been always presumed that its zeneral clirection, - that is, the direction of a line joining its base and top, -is vertical. Ihus, suppesing a tri-lineal composition enclosed in a right-angled triangle, such triangle wonld be standing on its point, with the hypotenuse, corresponding with the Rrimiful, placed vertically (sec: Fig. 1). There is, however, another style of design applied to a lange class of flower arrangements in which the Privelpal line of the composition has a hori zontal of almost horifontal direction: and, supposing a tri-lineal group of this style enclosed in a right-angled triangle, the hypotenuse of the triangle would lie horizontally (sce lig. 2). This latter style is chicfy applied to flowers arranged in hanging vesscls, or in standing vases when

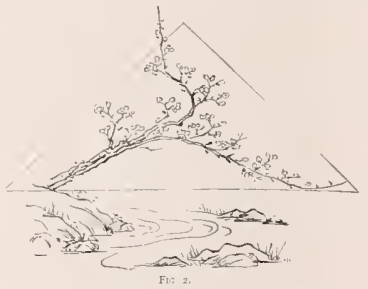
placed on raised shelves, the intention of unch compositions being to suggest foral growths on the eclges of cliffs or banks which lean ove laterally. In suci elevated artangements it often happens that one of the auxiliary lines on the sitle towards which the Principal bends over receives a decided droop and proportionate lengthening in order to emphasize the droop. Such dronping lines are tuchnically calked S/rcamers. The Streamer character may be imparted to either of the auxiliary lines, but redundancy must be avoided, and it is regarted as a fatal error to introduce Sircaners on both sides of the Brincipal. The Sreamer is very much used for foral arrangements in suspended vessels, in tall bamboo vases having mouths in their sides or in receptacies placed upon the edges of raised shelves. The prevailing idea in tach of these cases is that the composition must suggest the wild growth on the elge of a bask or precipice over which the Stramer droops (sec Plate XX111. A).

The ahove descrijtion has been confined to three, five, and seven-lined designs. Single line and donble line conpositions, as well as those of nine, eleven, or more lines, are sometimes maxle, Lut their use is very rare. The double-line form is emplayed occasion. ally fur the simplest of all flower arrangements, namely, that of one fower and one leaf.

The different lines of a composition have hitherto been spoken of as if existing in onv vertical plane parallel to the sjectator, but actually; in addition to the directions 


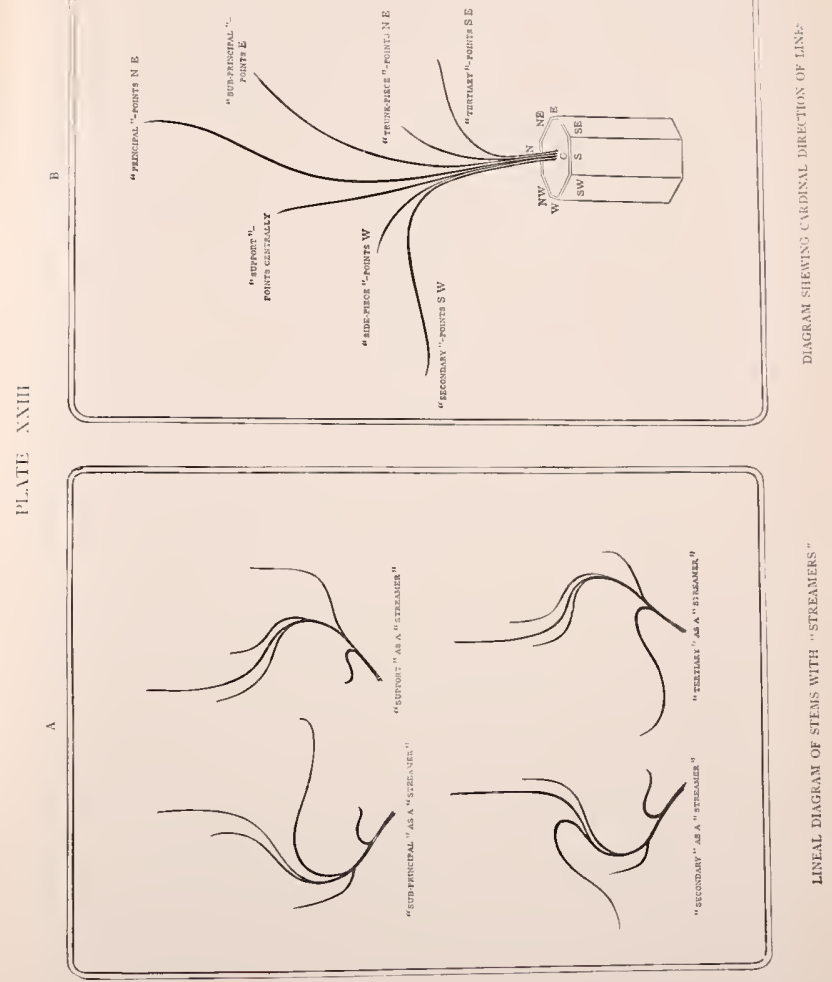



inentioned to the ight, left, upwards, and downsaris, thes lines hat alsu dir. ction of

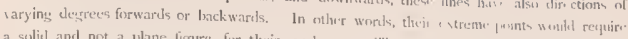
a suljel and not a plane figure for their enclosurc. Tliese diractions are best mplained by sipposing a bundlle of stems placed in a vase of octagenal plas, and designating the eight different facets of the vessel respectively as north, north-cast, east, soutl-endst south, south-west, west, north-west: then, imagining the south face of the vessit] immediately in front of the spectator, and bearing in mind that all the stems coincide for some little clistance from their origin. the cardinal directions which they take after scparation would be as follows (see Plate XXIII ns.):-The Primapal bends north-cast, the . Scondary south-east, the Teriary, south-west, the Sub-principul, which is between the Prinupal and the Tertary, turns cast, and the Sipptort, which lies Letween the Pronsipal and Secondary. terminates centrally over the vase. Of the two extra lines used in seven-lined arrangements, the Frumk-line bends north-east, and the Side-lime bends west. In this manner it pleasing variety back and front, as well as haterally, is given to the compositions, and they are not the flat arrangements which night at first sight be supposed from explan.tory drawings. Though designed principally with the object of bu ing seen from a point of view inmediately in front, they obtain by the above treatment solidity and varicty, and their effect when regarded from points to the right or the left are also taken into consideration,

Certain errors in arranging the lines of floral designs are pointed out to be strictly moicled. The first is that of Cress-cutting, which is produced by allowing two or more lines visually' to intersect one another, so as to form angles.

A similar fault, called Ticumentting, exists when a stem or twig cuts across its parent stem or branch: but this is allowed for certain trecs, as for exampll. the l'lum, with which such inter-crossing is a characteristic feature of the grouth.

Another error, called Parallitism, occurs when two or more adjacent stems or branches are cxactly parallel to one another, a fault much aggravated when the lines are, in addition, of equal or nearly equal length.

If indvo-culting, so named after the curved of cusped windows of the temples of the country, is the name given to an error accasioned by allowing stems to cross and re-cross in curves so as to form loop-like openings.

Latficc-cutting is an exaggerated form of Cross-culting, and this defect occurs when several stems cros's in parallel directions so as to suggest the appearance of lattice work. 
Another hatal error is that of employing the Donble stieamer, or drooped branch, on iroth sides of the same composition. The above rules of prohibition, it will be observed, are for the most part similar to those followed in foral and other ornamental designs in the hest styles of the architectural arts, being intended to prevent harsh, contlicting, or redundant effects and to be productive of general harmony of linc. They are illustrated in Plate XXIV.

The various directions of line imparted to the stems of plants and branches of trees on the above principles are obtined, frist, by a careful selectinn of stitable material: then, by twisting, bending. building together, and fixing at the base: and, lastly; by means of curting and clipping away defective and surerfluous parts. Some special methods of manipulation will be afterwards consjlered. 


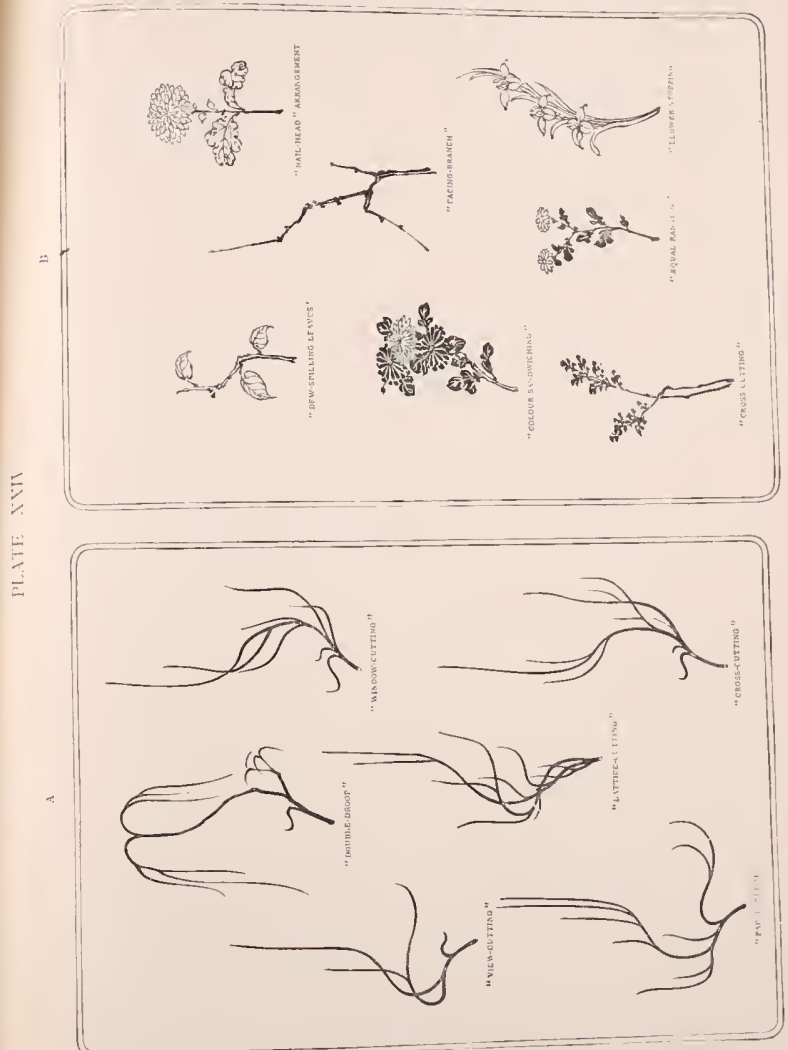





\section{SELECTION OF MATERIAL.}

\footnotetext{
$\therefore \rightarrow$ tos

PON the guntral lines of composition already indicated, flower arrangements are (45) J made, sometimes with one species of tree or plant alone, and sometimes with a the combination of two or more species. The use of many different kinds of flowers in one composition, though followed in the earlier styles, is opposed to the principles of the purer styles afterwards developed.
}

Combinations of two or three different species are, however, very common, and especially applied to arrangements in vessels having two or three openings. In all compositions, single or combined, the special nature of the different materials empioyed is carfully kept in mind, anything at all suggestive of the inappropriate being most scrupulously avoided. Important distinctions are made between trees and plants, and between land and water plants. The locality of production, whether mountain, moor, or river, considerably intuences the arrangement adopted. Each flower has its proper month or season, and many flowers which are common to more than one season have special characteristics of growth, of of form, during the cifferent seasons. These points of distinction are carefully studied, and are expressed in the artificial arrangements, within the limits of the art. Second flowerings, or flowers biooming out of their proper season, are discarded in flower compositions. As an example of this may be mentiond a late kind of pracis blossom, appearing in the Summer, which, beautiful as it is, may not be employed, because the peach blossom is specially a flower of the Spring time.

In arranging two or more species in one composition, variety must be sought by combining branches of trees with plants. In the case of three lines being used, the branches of a tree should never be "supported" on both sides by a plant, nor should a plant be placed in the centre with a tree arrangement on either sick. This faule is called by a term which will be better understood if freely translated as santwiching. In a 
trij] i. ormw ment it is phain that two branclies of the same kind of growth must be used, hut tho = aljoin, and not santrich the remaning one. As an example of a defective arrangeme , way be taken a composition with irises (ffants) in the centre, and branches of uralea and i nnellia (tress) on either side. A correct composition would be one with a plum lrand (lrex) in the ecntre, a pine branch (trec) on one side, and a bamboo stem (1, Wum in the other. For examples of the above correct and incorrect arrangements see Phate XXI: Sume Schouls allow occasionally the violation of this rule, and permit the whphrtung of atree on either side by a plant. provided that the tree is a mountain tree and that one of the plants is a land and the other a valley or water plant.

The above rules were no doubt made principally to prevent the weak and insipid arrangements likely to be producel by the careless viohtion of the principles which they express, especially in the hands of the inexperieneed. Plants, as before mentioned, are regarded as fenale with respect to trees, which are considered mak, because the former are weaker and more graceful in character than the latter. A slender plant flanked on either side by tree branches would give a weakness of effect to the centre of the composition, and the reverse arrangement wothl give too much strength to the centre and weakness to the sides. In addition to this, such arrangements would have a more or less synmetrical character, and symmetry is disliked throughout the whole of the art under consicteration. Like most arbitrary rules, such directions were often departed from by, the more advanced professors, and there are even recogrnized exeeptions which are universally admitted as correct. For example, two kinds of pine branches may be used tongether with a plum branch in a triple arrangement. Also in duuble compositions the willow and camellia are used togrether, and the Citosia argentia is often employed with the Sirissa fatida.

As previously stated, the branches and foliage of evergreens, and even of deciduingle blossom. It is, however, laid down as a general rule, that no flower-bearing plant time be used with flowers alume. nor must plants or trees which bear leaves at blosson leaved Chinese orchid has a tlower the following are exceptions to this rule :- The large plant is therefore technically treated as a llowery insignificant and grows below, and this arranged for its leaves only, before the flowers one. The Iris japonica is sometimes The leaves of the Summer narcissus, sanetimes appear, and it then receives a special name. plath, are facled and withered before the fonetimes called by the fancy name of Goldent pillar and cliscarded. 

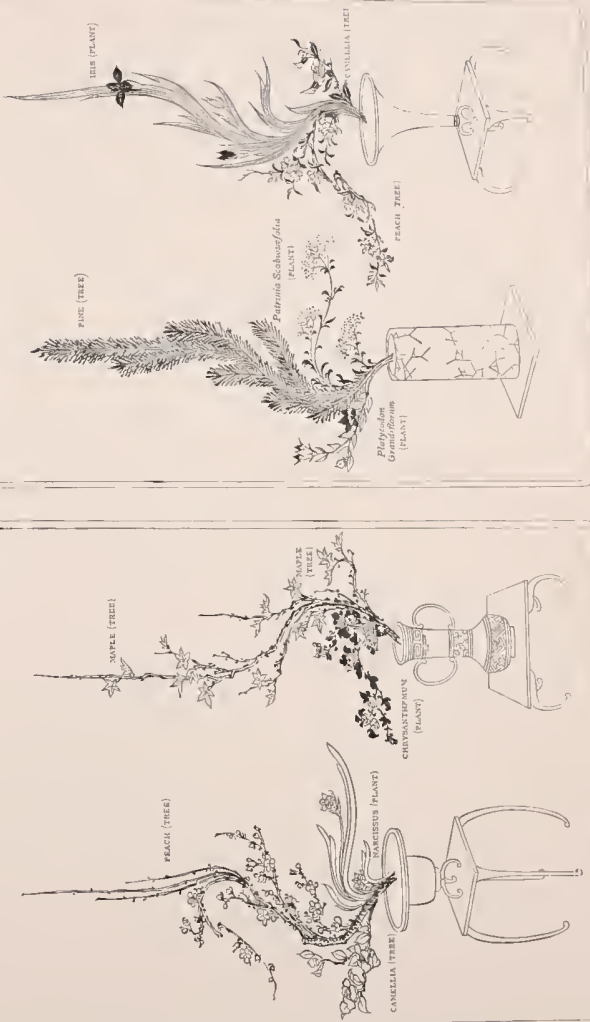

All flower compositions must partake as much as possible of the spirit of the seat wot in which they are used. Spring arrangenents should be strong and powerfis in line, like the growth of early regetation: Summer arrangements must be full and sprending: whilst those of Autumn should be spare and lean; and those of Winter, withered and ireary.

Mention has been made above of certain fatal crrors in combining the stem-lines of a flower composition, which are to be carefully avoided. There arc ocher faults in arrangements which concern the distribution of leaves and flowers, Large blossoms should not be allowed to occur in regular steps or rows in a composition, and this error is calied the Steppod arrangement. Another fault called the Nait-hect style, after the stitf metal flower ornaments used to cover nails in Japanese buildings, consists in a flower turned exactly full view to the spectator with teaves on cither side. The term colonr-calting is given to the crror of placing blossoms of the same colour on cither side of a single blossom of another colour; as, for example, a white clarysanthenum between two red onus. This is only another example of the prejudice prevailing against sastatichizg of any kind. The term Dew-dropping laf is given in a compasition to a leaf which droops down in a feeble manner so that it could not support a drop of water; this is greatly objected to. The above errors are illustrated in Plate XXIV.

Three distinct stages of vitality are observect both in flowcrs and leaves, In flowers, there are the full blossoms, the half oqen blossoms, and the buds: and in leaves, the young green leaf, the full leaf, and the rudening or falling leaf, In flower arrangements with one material, as for example with cherry or peach blussoms alone, a differcnt character of Llossom is selected for the chicf lines of the conprosition. For the Irimcipht, full blown flowers will be used; for the Sicondary, half open flowers; and for the Tertiury. buds are employed. Some designers, on the principle that the half open flower is more powerful than the full blown blosson, use the half epen flowers for the Primcifol, and the full blown blossoms for the Stcmatary. Straight leaves are considered stromg, and curled or bent leaves weak: the strong thowers slowld be near the weak leaves, and the strong leaves should adjoin the buds or over-blown flowers. A flower befow a leaf is weaker than one above. In thinning out leaves in a composition, two strong leaves must remain for every weak ane. 



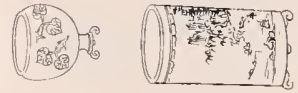

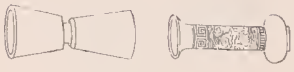

N11.
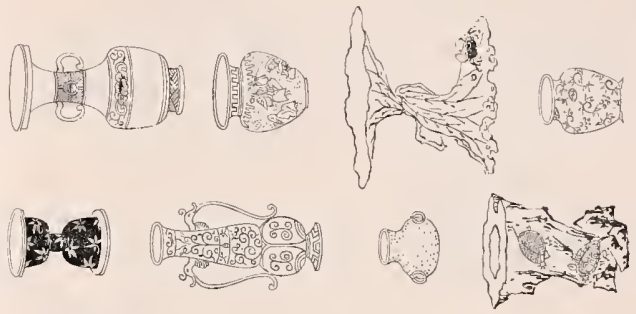

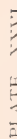
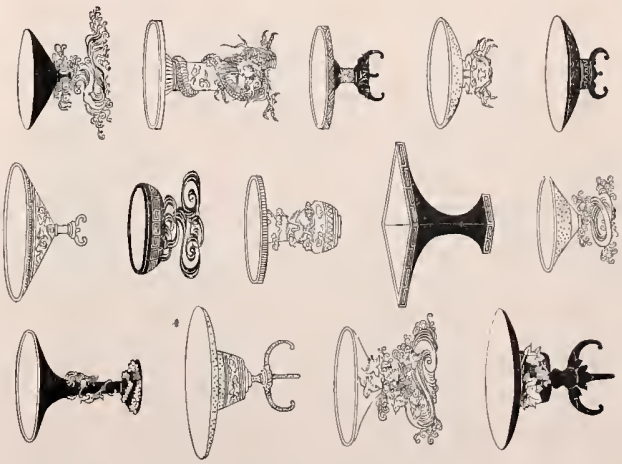



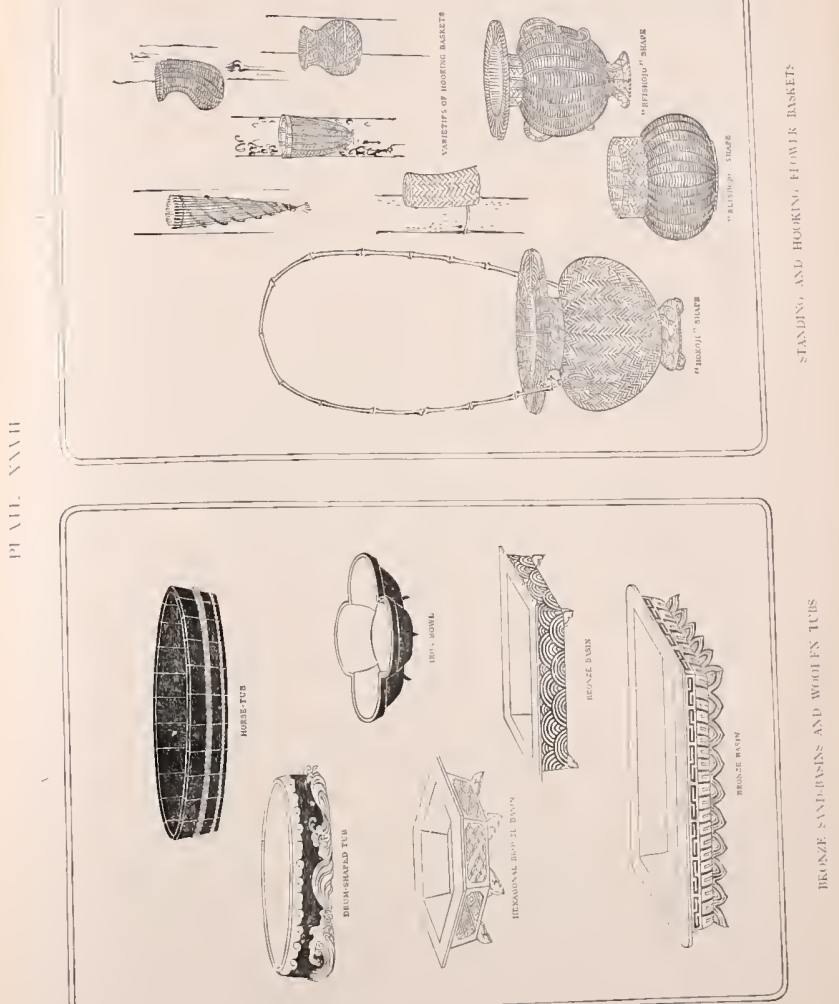





\title{
FLOWER VESSELS.
}

\author{
STANDINI; VASES
}

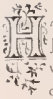

AV'ING clit ined the different plants and trees which supply material for the Japanese floral designer. it is neccosary to describe the various sorts of Hower vessels which are employed for receiving the compositions. The form and character of such vessels considerably influence the nature of the floral arrangements placed in them. These recentucles arc of a diversity of shapes and of different materials, such as wood. porcelain. pottery, bronze, brass, iron, and basket-work. Without attempting to trace the: development of the different art-industries as applited to vessels for receiving flowers, it will be necessary to refer to the various siapes, proportions, material, and decuration of such vessels, so far as they control or are giow incal by the foral arrangement adojtecl. The most ancient receptacle nacd was at long-neched enrthenware or bronze vase of considerable height, and the flower composition placel therein was made proportionately tall. The diffieulty of balineing stuh high arrangements led to the use of shorter and broader vessels and to a correspondling lowering of the compositions. This fuct shows that from the earliest times a recognizecl connection of proportion existed between the floral yrouj and the vessel in which it was jlaced.

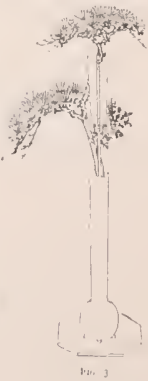

It is important to note that the surface of the water in which the flowers arc placed is techuically regrarched as the soil frum which the growth springs, and the designer must here convey the impression of a stable origin. With this principle in view, and gruided also by the necessity previously mentioned of avoiting too great a height in flower 
compositur it wrt of broad-monthed vase became the favourite form used, Uf this kincl there are many shapes, generally in bronze, and vary ing from a low saucer-like vessel to a trumpet-shaped form, supported sometimes on short legs, and sometimes upon ornamental castings representing stch suljects as rocks, water, spray, or an animal group.

Other kinds of vessels, curresponding more to the orlinary vases employed in the West, hasing urcks and oval or cylindrical bodies, are also much used. Among these, the vases widh tall narrow neclss are enylloyed for the simplest arrangensents, such as are displayed in the tiny tea roms where rules of austerity necessitate very light and quict floral compositions. Vases intented for elaborate flower arrangements are always necussarily of the widc-mouthed kind, to ensure an appearance of stability at the base of the flower stems. A few of these different wases are shown in Plate XXV1. but there exist a number of other shapes in common use, besides sevtral fancy forms which are occasionally employed. With the ordinary tall vasc, whether of svicle or narrow mouth, the height of the flowers is generally. fixed as appromximately one and a half times that of the vase, Some Sehools increase this proportion, giwing double the height of the vase for the Hocal composition.

\section{VESSIIS FUR WATER PL.INIS.}

For the purpose of displaying, in a suitalule and suggestive manner, water plants and rasses, low tub-like vessels came into use from quite early tines. There are two principal kinds employed, called respectively the Simat-bual and the Horst-fub. The Samt-bund is a broad shallow vessel, oblong, polygonal, or sometimes oval in plan, nade of bronze or porcelain, and contaning a layea of pebbles or sand covered with water. The Horse-1ut is, as its nane implies, a shallow tub of wood, circular in plan. and gener .lly lacquered black. Its use as a hower vessel is said to have originated during the exigencies of war time, when a fimous general of iesthetic tastes, in the leisure of a lung campaign, employed a common horse-tub for arranginu flowers in. Whereas it is a rule with other kinds of flower vases that the witer shall not be visible, the water in vessels of the Somt.bont and flow tub kind is intended to form part of the composition. The idea to be conveyed is that it a bronl surface of water or marshy ground, and the plants and trees used are only those which are associated with the propinquity of water. Water plants are chiefly emplojed, but 


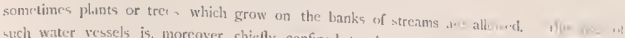
weh water ressels is, morcover, chiclly confined to the warmer month of the ge.tin when the sight of a broad surface of water is gratelul and reffeshing to the eya. A different rule of proportion between the flower cumposition and the vessel is followed when these broacl shallow receptacles are employed. The height of the floral comprosition is made: alout one and a half times the breadth of the vessel, and is not regulated by its $h \mathrm{cig}_{\mathrm{g}} / \mathrm{t}$ as with the ordinary standing vascs.

It is customary with the above-named water. lowk to use fancy fasteners of metal, to holk, or give the appearance of holding, the base of the flower stems. These will be describel afterwarels when the whole question of fasteners is discussed. Examples of Sindbrats and Iforsa-tubls are shown in Plate XXVll A. A special water vessel requiring mention is that which goes by the name of the Leng-hurt, not from any particular resemblance

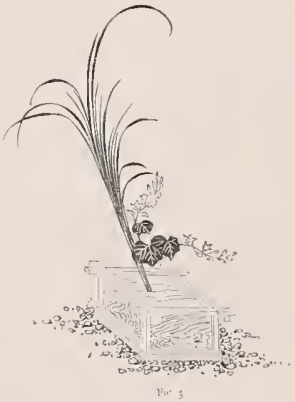
in form to a boat, but from the fact that it is very long in proportion to its width, and is made of white wood. In shape, the Loms-boal resenules a Sirnd-bure. and it is uscd in a similar manocr, with sand, pebbles, rocks, and water.

Another form of standing vessel sometimes used for plants is the II:Hframe, a sont of box-shaped vessel of worm-eaten wood, with a eapping piece crossing at the angles like an Oxford-frame, the whole resembling the wonden framing round a Japanese: well (see Fig. 5). The rustic character of this kind of vessel requires that the Howers placed therein should be of the simplest kind. In the example illustrated, a plant called Togiri (Clerodendron squamatrom) is used in combination with a long slender grass called Susulit (Lntario jafinica). 


\section{FLOWER BASKETS.}

The famoun Regunt loshimasa is said to have been the first to mploy the plaited basket as a receptacle for flowers. Raskets, made of recdis, stens of crecping plants, cane, or bamboo, of Chinese manufacture, were much prized in Japan, and high

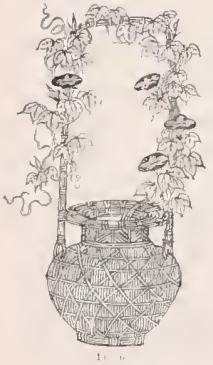

the water, which are bidlen within.

Another popular story, in connection with the use of baskets as flower vessels, is that the famous philocopher Rikiu started the fashion on the oceasion of a visit to the chersy blossoms on the banks of the river Katsura. near Fioto, when he made use of a common trout-backet in arrange gathered flowers in. There is a special backet still in use

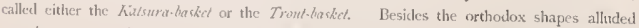
to, there are various fancy forms of haskets employed in both standing and hanging ponitions. Of these the following principal examples may be mentioned:-

The Hatf-plailed-basktt, a cylindrical basket in which the vertical threads project two or three inches above the horizontal plaiting, as if unfinishod in making.

The Rnun-chat-binktel, a hensispherical basket with a frayed top hanging over the edge of the mothl, and somewhat resembling the straw collar of a Japanose furmer's raincoat. 


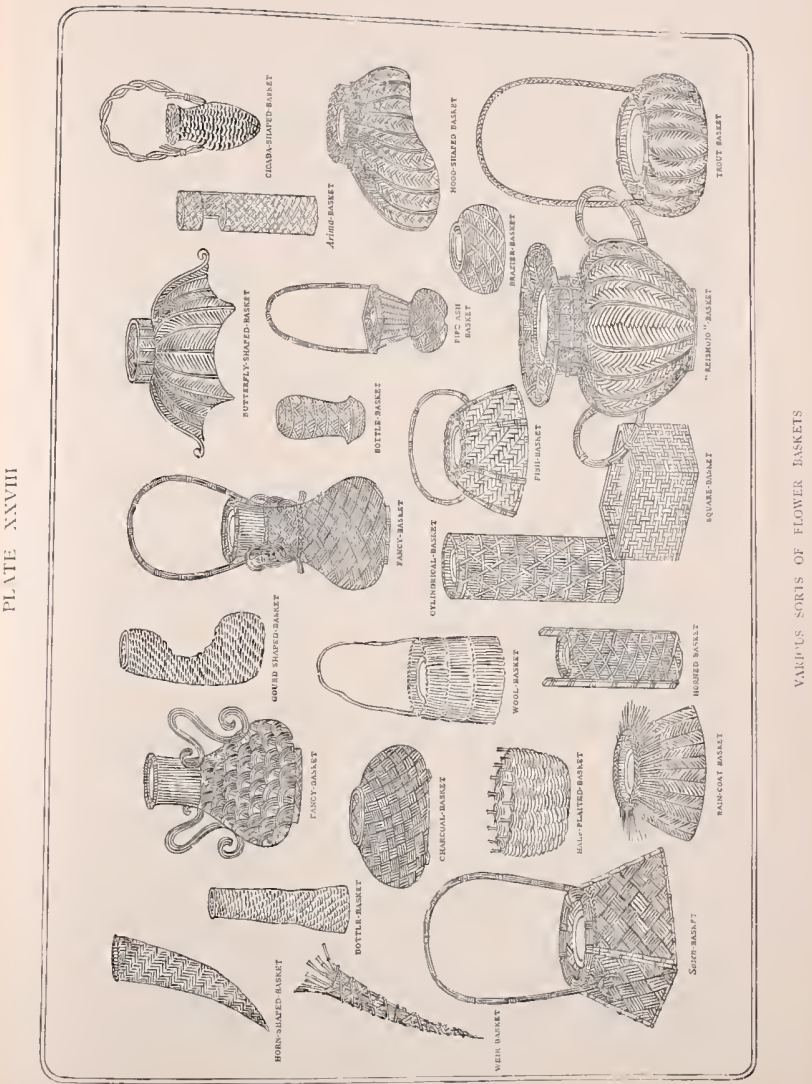





\section{FLOIFER IESSELS}

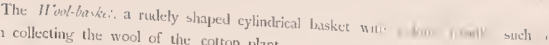
is used in collecting the wool of the cotton plant

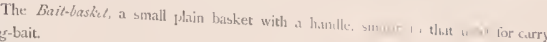

The Iformot-bisket, a basket of tall cylindrical form, with two hom-like projections on the top as handles.

The Symare-basket, a basket of simple cubical shape with no handle.

The Loun-bristict, a tall, thin, tube-like basket.

The Sirate-bakkt, a basket of pyramidal shape, having four sloping sides gathered to a circle at the mouth, and with a high arched handle. The nane sosen is that of its inventor. It is shown arranged with chrysanthemums in Fig. 7 .

Amongs special baskets for hanying or hooking may be mentioned the following :-

The Horm-shapot-baskit, a basket shaped like a bull's horn.

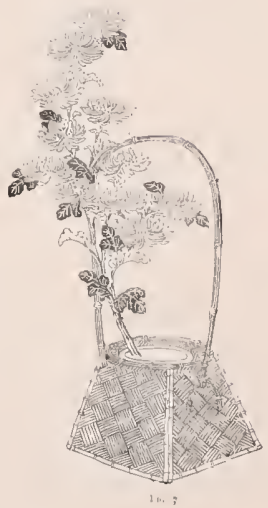

The Cieadia-shapal-brestirt, so called from its resemalituce to the body of th insect.

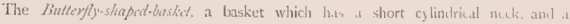
broad arched boty curving out to points at the botton, the whoke form being sugatentm of a butterfly with open wings.

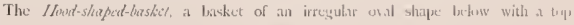
opening towards one end, somezwat resembling in shape a biby's sock and, to the 


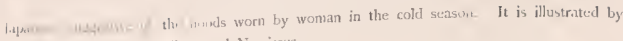

Figr. ariangr t woth Willow and Narcissus.

The Giserd slatein birbit, a basket woren in the form of a gourd.

The Durikokn's-buts-bustich, a basket retenabling in slaps the bay carricel by the Japan. ese God of Riches, Daikokir.

The Irima-bustiet, a busket of cylindri cal form with a sikle imotits. similar to the hanging lamboo basket. The name is derived from a villate called Arima.

Ior certain susperded arrangements of creeping plants a deep net-work basket. something like an English waste-paper basket in shape is often used. Willy this a low flower vase is placed from which the creeper rises, being arranged so) that part is scen through the netting of the basket, and part hangs over the side in a slmamer or trail. In all cases flower baskets require an inner vessel for holding the water in which the flower stems are ptaced, and this generally consists of a concealed tube of bamboo. Examples of various liowet baskets, including those above

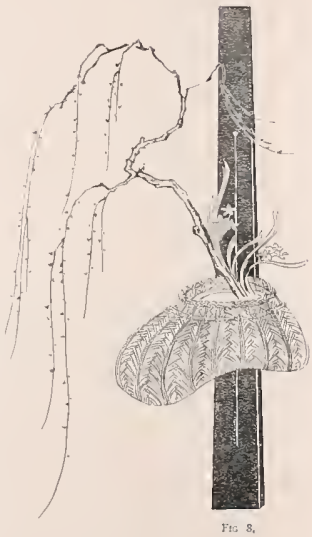
deseribed, are to be seen in Plate XXVI, and Plate XXVII,

\section{B.AMBOO VASES,}

To Yinshimasa's patronage is also attributed the original use of flower vases formeil out of bamber tules. As first introduced, these were simple cylinders of thick bamboo, cut near the root, about a foot or more in lecight, and four or five inches in diameter, the teotom being clomed hy a natural division. The facility with which such vessols coukl be. 1 ut liato different slapes led to the invention of a variety of forms, each bearing a fancy 


\section{FLOHER TESSELS.}

name and specially atlapted I., different shyles of flower arrengentm nt. Portions of the sides were notehed out, and side apertures were introduced, often in several stages, so as to allow of two, threc, or five compositions in one vase. The different kinds employed are so numerous as to require tabulation, and many of the names alnost defy translation : they

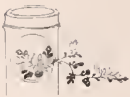
are therefore given in Japancse: The meanimgs of these names art explained as far as possible, and it will be seen that they refer chiefly to some fancied resemblance in gencral shape, of in the form or number of the apertures, th other native objects. They are as
follow.

Shishiguchi, satur,-Lion s-mouth-shape: a cylincter from ten to fifteen FIC. . inches in heiglit with a square side-month about 3 inches deep.

Acherijishi-geta.-Rampant-lion-slape, a higher cylinder with side mouth as above,

Tabumakura-gala, - Travelling pillow-shape: a very short vase with mall side slic

L'atiguchi-gata.-Singing-mouth-sibape: so numed from a sydyed form given to the side opening, suggesting the incline of the lips in singing.

Wanguchi-gatu. - Shark's-mouth-shape: so called from tucth like proninences given to the bottom of the side aperture.

Fukurokuju-satu, -Named after a Japanese magician represented with a very ligh cranium, on account of the low position of the side month.

Airmomon-gala.-Chinese-zateway-shaye; a high vase with one of the side openings rounded, resembling a Chinese arch.

Gommen-gath- Wild-geese's-gateway-shape : a vase with curved side-opening somewhat like the above, the name being taken from thit of a famous arched seit rock called the Wilitgerie's-gaterive:

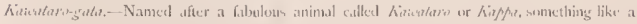
monkey; and said to inlabit lakes. 


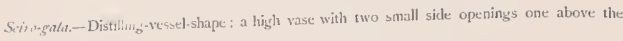
other.

Aizinma-gatu.-Tile-shape; so called from its resemblance to a half cylindrical capping tile.

Ro-gala-Oar-blade-shape: resembling the blade of a Japanese oar.

Torogala-Lantern-shape; supposed to resemble a stone standard-lantern.

Awho-gatis,-Ray-fisli-shape.

Daruma-gata,-Seated-hermit-shape: this vase spreads out at the bottom and has an arched side-opening near the toy, suggesting the form of a lernit's cowl.

Noborizarm-gaia.-Clinbing-monkey-shape; this is a high vase with a very long deep cut in the middle, leaving two short cylinclers at top and bottom.

Daibu/su-gala.-Scated-Buddha-shape; so called from its suphosed likeness to a seated Buddhist statue.

Lirko.gatia,-Monkey-shape.

Kahguchi-gala-Cuspect-opening-shape; so named because of the caspet form of the sideopening.

Tora-gata,-Mantis-shape.

Gojin-grala.-Fire-storey-shape; a very high vase with five side openings.

Hokci-gath.-Icicle-shape; so called from the leg-like cuttings at the botom of the vase in the shape of icicles.

Mitsmashi-gaha,--Three-legged-shape; in this vase the bottom of the cylinder is cut away leaving three legs remainitig.

Torikigy-gala.-Bird-cage-shape: 

Troke-gatit.- Hand-pail-shape; resembling a Japanese hand pail, two decp apertures bring
cut exactly on opposite sides so as to lenve a handle-like strip above.

Teginc-galat-Pestle-shape.

I'su-gala.-Mortar-shape. Shakuhachi-gata.-Flute-shape: a long thin tule of bamboo, slightly bent like a native
flute.

Hashigui-gata. - Bridge-post-shape; supposed to resemble the newel of a wooden bridge rail, having a deep square slit in the middle.

Wiolsnkushi-gata.-Beacon-light-sbape.

-Viju-giri-gafa.-Two-storey-shape: a vase with two side openings one ahove the other, in addition to the top opening.

Siajiu-gini-gatu. -Three-storey shape.

Tsurubc-guta.-Bucket-shape; named after its supposed resenblance to a well-bucket.

Tsurnkinbi-gala.-Crane's-neck-shape: so callecl from the length and depth of the side cutting which leaves a long thin neck of bambon suggestive of a crane's neck.

Tsurigane-gatu.-Bell-shape.

Koma-gata,-Spinning-top-shape.

Tarai-gata,-Tub-shape:

Heragai-gata.-Conch-shell-shape.

Taki-nobori-ria-gata.-Cascade-ascending-dragon-shape : a bigb bambon vase cut into a long spiral, supposed to resemble a writhing dragon. 


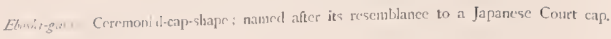

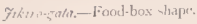

Aijiu-yugura-gath.-Two-storey-caste-turret-shape: so called from square embrasure-like side opunings near the top.

Hateumu-grati.-Pigeon-brensted-shape; so called from a bent in the bamboo cylinder giving it a pigcon-breasted appearance.

Ribktan-gala,-Pan-pipershape; a row of small bamboo tubes of cliferent heights tied together with cord and fixed on a stand.

The invention of most of these bamboo vases is attributed to different professors of the Tea Cerenonial. As will be perceived in the above list, assisted by the illustrations. the breadth, depth, and roundness or squareness of the side apertures, as well as their minber, and the total beiglat of the vase, suggest the nanes for the difierent vases, Mhny of them are provided witls a circular nail hole on one side, near the rop. for lrangings purposes, and such vessels can be used at option, either looked to a nail or standing upon the floor of the alcove. The tall kinds having open tops are invariably used standing.

Another varicty of banstoo vase not previously mentioned consists of three or more bamboo cylinders of different heights attached in a line, and named THe Rout-nf-piles, after their resemblance to a row of pile heads. Many of the above mentioned bamboo vases are illustrated in Plates XXIX. and XXX.

There also cxists what is called the tirdant bamboo ansc, being a vase of one of the above shapes, fresbly cut from a growing bamboo stem, with twigs of green leaves remaining on it. In such a vase the intermediary knots or divisions are left intact, and small apertures are introduced is the side for filling in water and other preservatives against
spredy withering. Japanese flower vessels may be broadly diviled into three kinds, those used
for standing upon a dais, table, or shelf: those intended for hooking agrainst the wail or
against a pillar: and those suspended by chains or cords from ceiling against a pillar: and those suspended by chains or cords from a ceiling or beam. The vessels hitherto described belong to the sfanding kind, with the exception of some of the baskets and certajn of the banboo vases just enmmerated, which can be used either for 

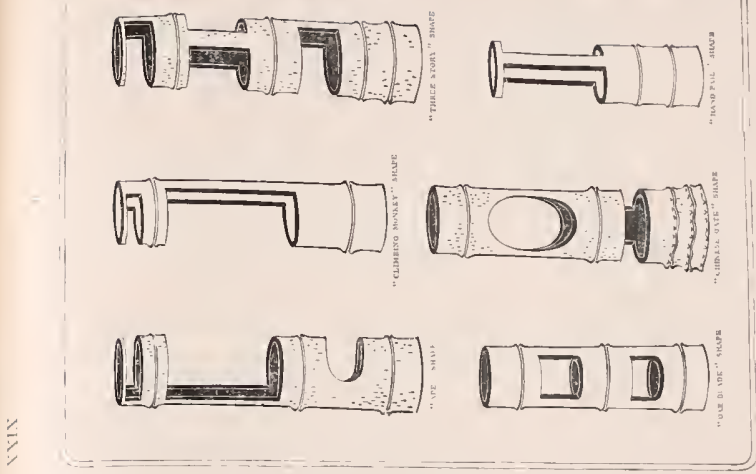

$\overline{1}$

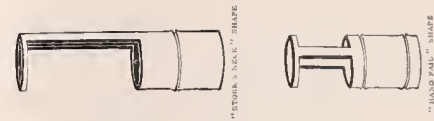

(1) UचD:
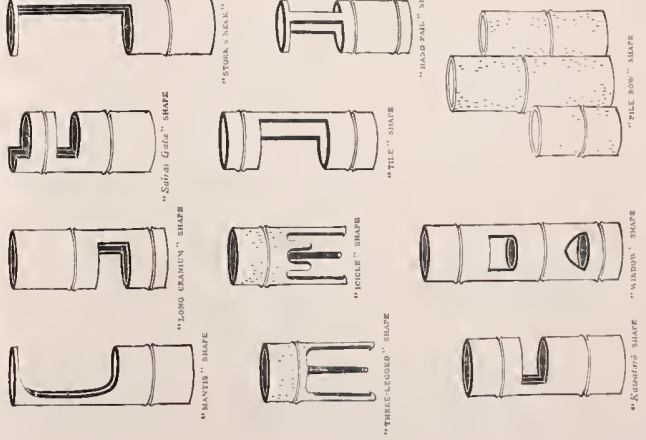



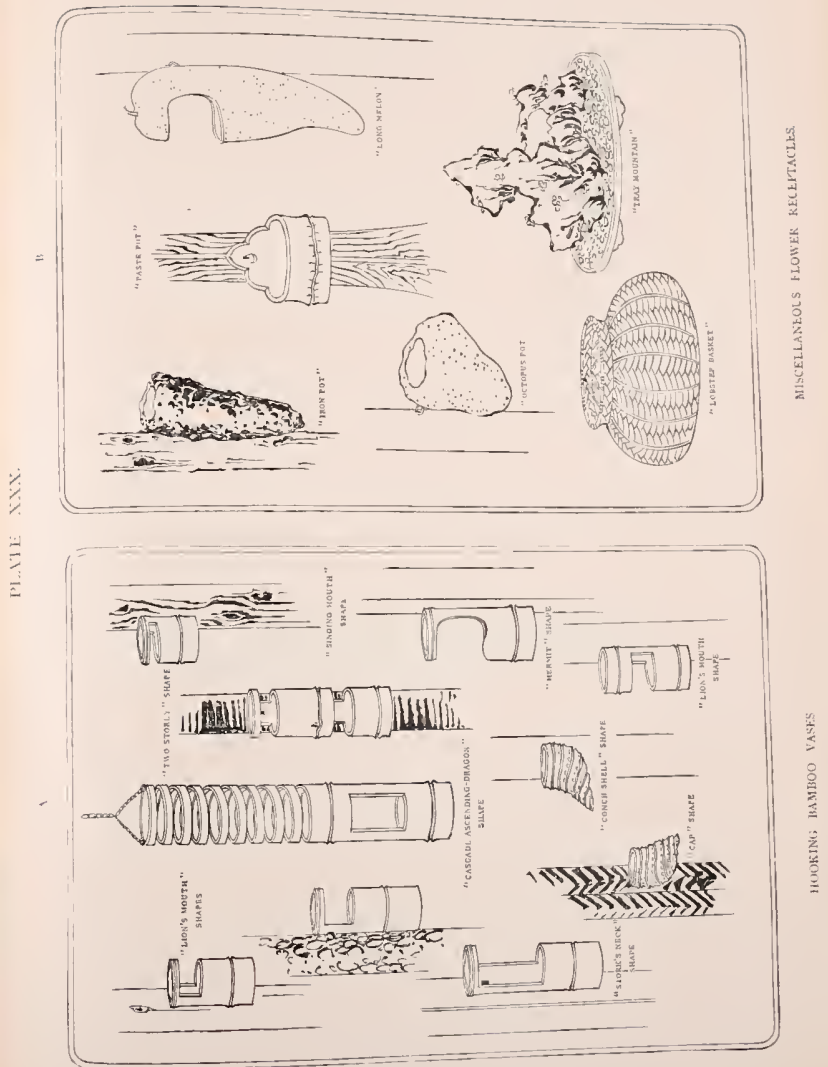





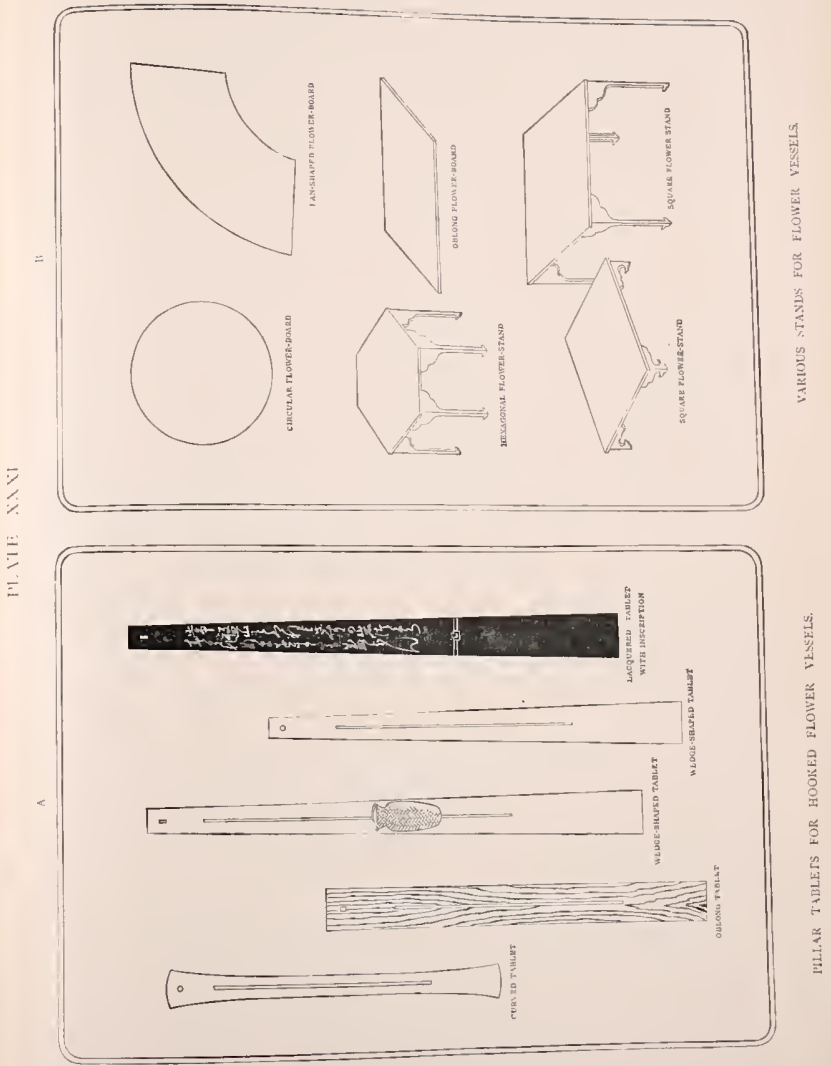



randing or liangine In order to distinguish clearly between flower vase which are Itooked to a pillar or wall surface and those which are hung by chains or cords, the former

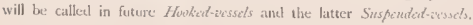

\section{HOOKED YESSELS.}

Hooked-iessts are of various kinds, from the chrysalis-shaped root of a banbon, 1... the form of a sleetl, gourd, or mclon. They are invariably short, comparet with the standing vases, and with a few exceptions, the absence of llatness below suggests their method of use. Among the banber vases, those of little height ant

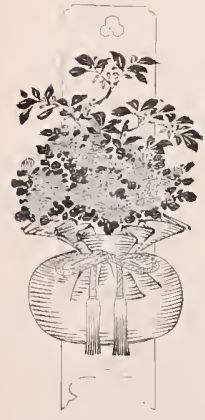

Fi5. 14 with narrow side apertures, such is the Lion's-mionth-shape and 'Fravtling-pillon-shape, are used mostly as houked vases. A haterul direction is given to floral compositions placeel in hooked ressels, the idea suggested being that of flowers banging over a cliff. For tea rooms, where a severe and rustic style of flower composition is peeforrect, vases of curious slapt are pressed into the, among which may be: mentioned:-the Celopus pol, a couse irreyular shapeal carthenware jar used by fishermen for holding the octopus: the lrm-pot, a rough iron pot-shapel vessel somewhat like a martin's nest, and the Decaycot-stump, a piece of decayed wood hollowed out as a vane. The Goned is alses a faynurite form for hooked vanes, the mesth beims sonetimes cut in the side and sometimes at the top. It is said to have been first used for flower arrangements hy the philosopher Rikiu, who once extemporized a floral clesign in a wine-grourd which he touk from an itinerast priest at the temple of Sumiyoshi near Usaka.

As previously mentioned, many of the woven baskets $\mathrm{cm}$ ployed as flower vessels belong to the hooked class. These are to be found deacribed under the head of flower-baskets, and are illestrated in Plate XXX.

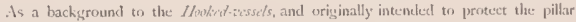
or prepireal wall-surfice from staining or abmaion, narrow oblong tablets of woct ar: often used. They are sometinxes ntacle ornamental, being lacturered and inscribed with verses in gold letters, Some are plain oblong tablets about four inclies broad and three or four feet 
long - others are welge-shaped, tapering towarcls the top; and others lave curved sicles. They are provided with a long narrow slit down the middle for sliding to different heights over the iron nail or pey by which they are leld to the wall or pillar, and to which the flower wase is hookesl. In seme cases these tablets are hinged in the middle to allow of folding up when sut of use. They are often made of segments of banboo tlattened out and polished or lacquered, Examples of these hanging tablets may be seen in Plate XXXI.

\section{SISPENDED VESSELS.}

Sirspested etessets are those hung by a cord or chain to the ceiling or lintel of a recess. Belonging to this class is a crescent-slated vase of pottery or bronze called the Crescint-moon. The horns of the crescent are made almost to meet and are suspended

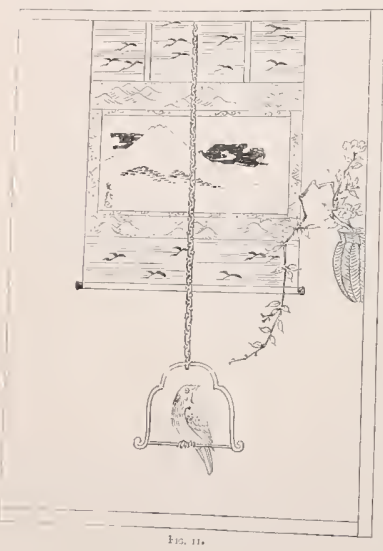

from above by a connecting ring and single chain. The other kinds being of more elongated form are hang fram bot/s ends by double chains or cords. The simplest of these are bamboo tubes splayer off at the ends, hollowed out in the midelle, and hung horizontally, so as to suggsest the form of a boat of punt; others are of bronze, shaped in exact resemblance to a ship or junk. loshimasa is said to have conceived the idea of boat-shaped rases whilst obsurving children sailing toy boats filled with flowers. Another story attributes the first use of such vessels to the famous philosopher Soami, who on a hot summer day, to please his patron Yoshimasa, took a bronze vessel of accidental resemblance to a boat, and by his manner of arranging the stems of the flawers therein, conveyed the 


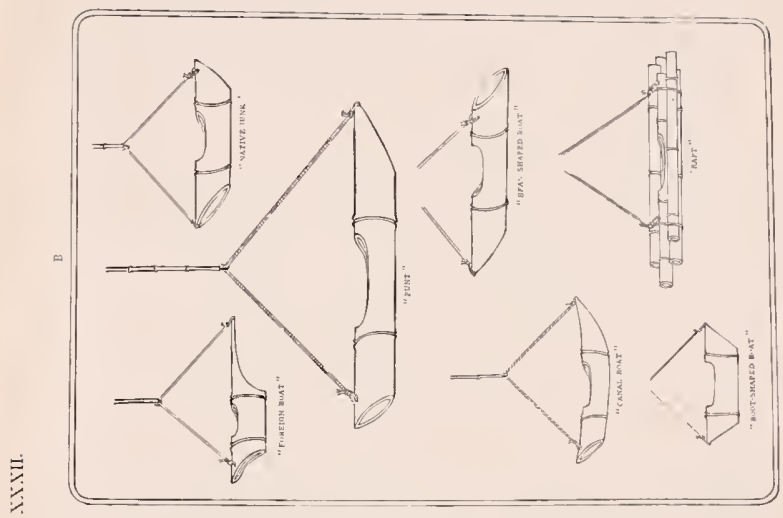

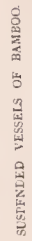

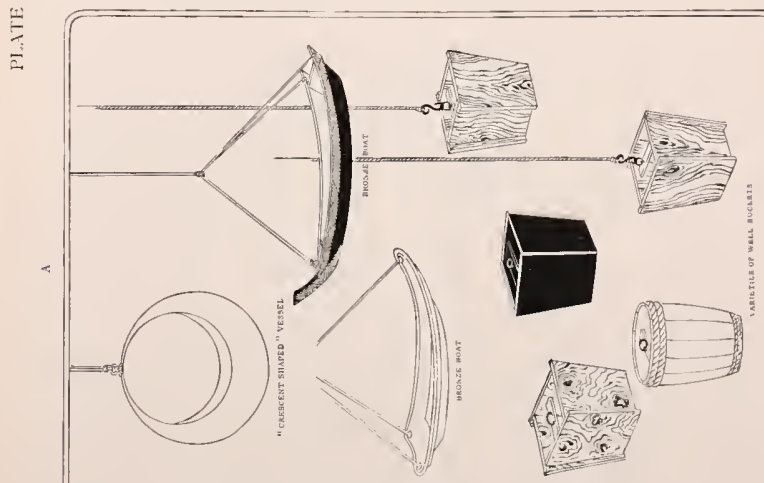



an unchor of black metal hanging to the side; the Basket-anerk-loal, a boat of metal basket-work. Also a curred cane-work tray, oblong in shape, hung from the ends, and carrying in the midclle a little howl of flowers, is sometimes used.

An important theory in hoat arrangements is, that they ought always to be susperded in an eterated position, lontly with a rjew to preserve the idea of a floating vessel, and also to prevent the possitbility of sceing the water which they contain. It is helel to be a great violation of taste to allow the water which is necessary for preserving the phants in a llower-boat to be seen, becallse, water visible within a ship would be suggestive

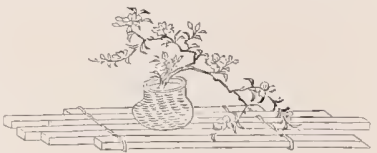

Frin, 33 of a leaking or wrecked vessel, and would be consequently considered unlucky: Sometimes the idea of a stranted or heached boat is purposely conveyed by a flower vessel which is placed upon the this instead of being suspended. In this case the vessel should be raised upon a stand of some kind so as to place its upper sutface above the eje level of seated visitors. Such standing boat-vases are supported upon two wooden rollers or upon a light frame of cross-picess. Before leaving the subject of boat-shapes, allusion must be naake to a standing vessel called the Lomp-linet which is sometimes employed for elaborate arrangenents of plants and grasses. This vessel appears to be called a boat simply on account of its narrow length and the fact that it is made of plain white wood. It resembles a Sind-ford, being an oblong tray-like sessel with short legs. It is fise feet long and about one foot wide and is only used for very large recesses on special occasions. The boat-vases clescribed above are illustrated in Plates XXXII. and XXXIII.

The classification given refers only to the different shapes and sizes of the boat-like vases. Other terms are used to designate the mamer of hanging the vessels and of arranging the flowers within, so as to convey different nautical ideas. The three principal arrangements are those of the Onhard-bonth-ship, the Honcuard-bound-ship, and the Ship-int-porl. Besides these there are other designs known as the Distant-shifp. the

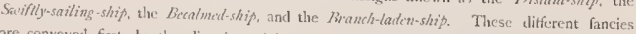
are conveyed first, by the direction, rights or left, and backward or forwitrl, given to the prow of the vessel: and sccondly, by the distribution of the different lines of the flower composition. Even the lengrts of the suspending chain and the distunce or proximity of 



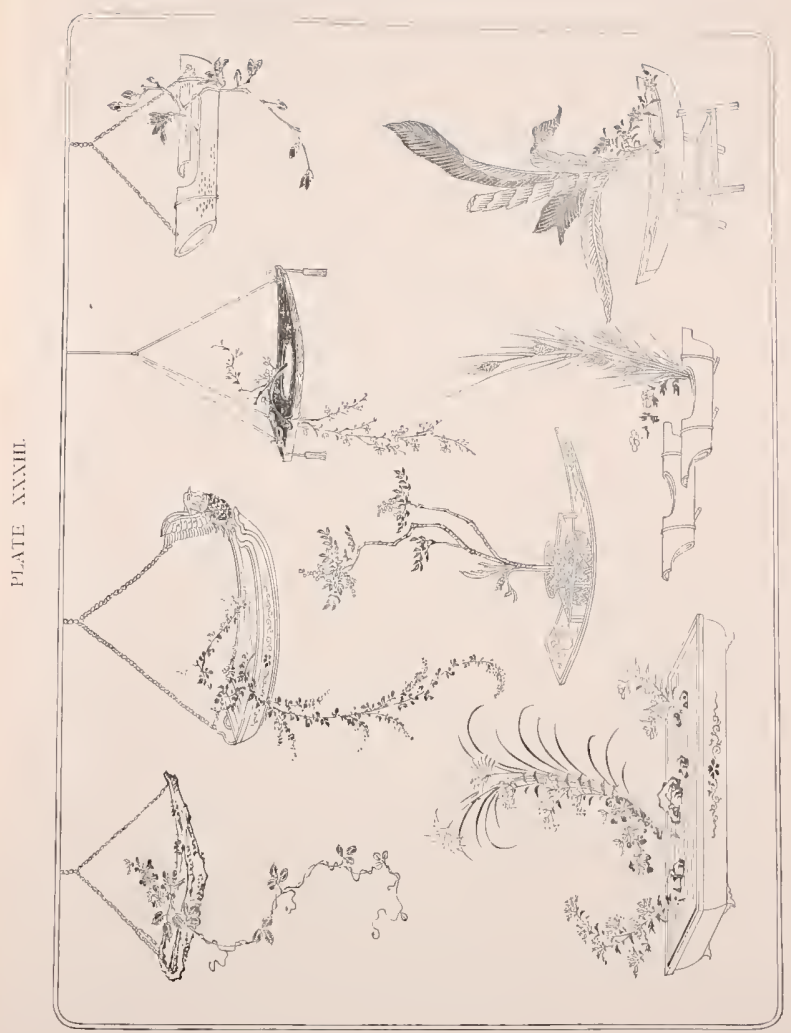


These fifferent styles of composition are shown in a skckton form in Plate Xx.xul: Otier special rules for hanging bnat-vases will be considered afterwards, when the grenural question of the position of flower arrangements in a clamber is discussed.

Suspended wessels calied Hith-buthits are often used in pairs hung over a pulley by a thick silk cord, One of the buckets is allowal to rest on the floor, or in some cases upon a frame designed in initation of the railing or boxing found a well. and the other is suspended in the iir.

To Rikin is attributed the first use of such flower vessek, the idea coming to him whilst he was observing a convolvulus twining round the bucket of an old well. A similar pair of buckets are occisionally ensployed without the pulloy and suspending rope, one being placeal balanced on the elge of the other so as to leave only a portion of the lower one uncovered for the insertion of flowers. In this case the rope is arranged in a flat coil as a stand for the lewer vessel. Buckets used in this way are always flat-sided, to

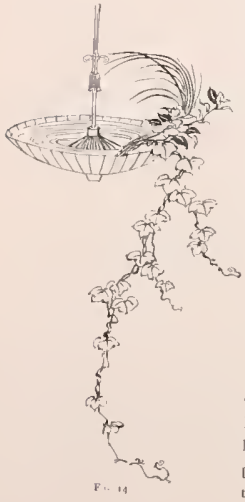
ensure stability; but for the ordinary suspended arrangements, cylindrical as well as square buckets are employed. These vessets axe of plain woml, of wood lacquered black, or of wormeaten or decayed timber. For the most handsone kind in black hacquer, a chain of sitver or a ral silken cord should $I_{x}$. used; tor those in ordinary wool a plainer cord may be attached: and in the crise of the rustic buckets of decayed wood, a comminn leomp rope or even an iron chain may be substituted. Singic buckets are occasionally to be seen standing upon a low table or dieayed slab of wood, or hung by a single busmboo rod. (Sece Plate X.XII.

Porcelain buckets and pulleys, although not uncommon. arc of quite modern introduction, and not according to rule.

Other fancy vessels suspended by cords or chains are sometimes employed. Among the bambon vases in Plate XXX.i. will be seen one which is suspented by a chain, like a lantern. Suspended baskets, listinct from the hooked baskets pretiousily described, are not uncommon. Another examyle is the suspended net-work basket previously' descrilxed. 
the arrangement from the observer is governed by the style adoptecl. According to the principles of lineal Jistribution which apply to all suspended flower designs, the Strenmor holds an important place in the above examples. It is in such cases intended to suggest the long bent oar which in Japanesc boats tails back towards the stern. This floral line must not be too powerful, as it represents the iden of an oar dragging in the water. Tho central flower stem stands for the single mast of a junk with or without sails, and the subsidiary stems indicate the other sails and rigging of the vessel. This will be beter understoot by describing one or two of the arrangements in dutril.

Hemerard-bound-ship. In arranging in hou of flowers in this form the prow of the vessel is turned towards the left, which, in superior rooms is the host's side of the: chamber, in order to convey the idea of home-coming. The central stem of the floral arrangement is high and full, curving towarls the helm, so as to inclicate a ship in full sail. and a Siremer hangs over the front side sloning back towards the stern on the right. The above is a favourite device on occasions of rejoicing for a safe return, or when a son or daughter-in-law is being received into the famly. Some say that this methoul of arrangement should only be employed from noon till clusk.

Ontumit-bunnd-slip. This is an arrangement exactly the reverse of the former: the vessel having its prow turned to the guest's side of the chamler, on the righth It is adofted at parting gatherings, in token of wishing good-speed to those secting out on at joturney. It is said that this style of composition should be employed only from morning till noon.

Ship-in-port. In this arrangement the vase has the same direction as thu: Homsward-bound-ships. but the floral design is kept small and straight, so as not to suggest wind or motion, and the Streame\% hangs over the further side of the vessel. Siuch at disposition of the thowers should not be mak excepting during the hours of cvening.

Suritly-sailing-shif. The direction of the vessel in this design is to the right, or ontwards: the floral arrangement is full and bent, but no Sircamer is used.

Iranth-laden-shif. The direction of this ressel is inwards, or towards the left. and the floral arrangement is kept short and close, and consists of small flowers, such as daisies or carnations, which are not allowed to projece locyond the limits of the vare: itsclf. The idea suggested is that of a ship loaded with timber or tree branches. 
with four $1 \%$. whels and no shafts, like a child's toy-cart, bearil a small bucket in which the flowers are arranged.

\section{JLOWER FASTENERS.}

The subject of Fatcustr for Horal arrangements is one belonging to the Wechnique of the art under consicleration. To a great extent, lrowerer, the methock of fastening are treated as a part of the decoration of the compositions, and as such they are dosely counected with the different flower vessels employed, and require notioc in the jrestent context.

As before mentiesect, the sprumbing or penint of origin of the thoral group, is of great importance, and the firm and skilfful fixing of the stems or brandres in the ressel which holk them is one of the most difficult parts of the manipulations. Ordinarily, the stems are hatd in position by: small cylindrical pieces of wnod fitting tightly across the neck of the llower vase, and having a slit, wider abowe than below, for threatling them through. The treclge-shapeal forns, wider towarts the top, which is given to the slit, allows slightly different inclinations to be imparted to the several branclues. The fastener should be fixed alout half an inch below the surface of the water. lise level of which is made to vary acoreling to the scasum, and it should not be visilde from the front of the vessel. If the vase used loe a hacquered one. paper slontle be placed between its surface . and the end of the fastener to perent scratching. In some lirge-mouthed vessels, and

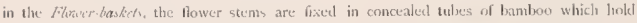
Whe water and the fasteners. Some Schools affect a rustic simplicity in their appliances and cmploy a maturally forked twig to hold the flowers in position.

For arrangements of water plants in neckless vessels such as Sint-boats or shallow Fubs, other sorts of Firstemers are necessary. which are hiden betow the sand or petulules which such vessels contain. One kind consists of a sheet of eopper perforated with holes of different sices to receive the extremities of the different stems. Another Frstimy is made of rings or sections of bamboo of sarying diameters attached to a watshen board, the steins finding loelgnent in the sockets thus formed, and being further leted in pusition by the pebales which coter them. Occasionally a Fastimer called the Whirlpeot, and consisting of a spiral hoop of metal placed vertically, is employed. 


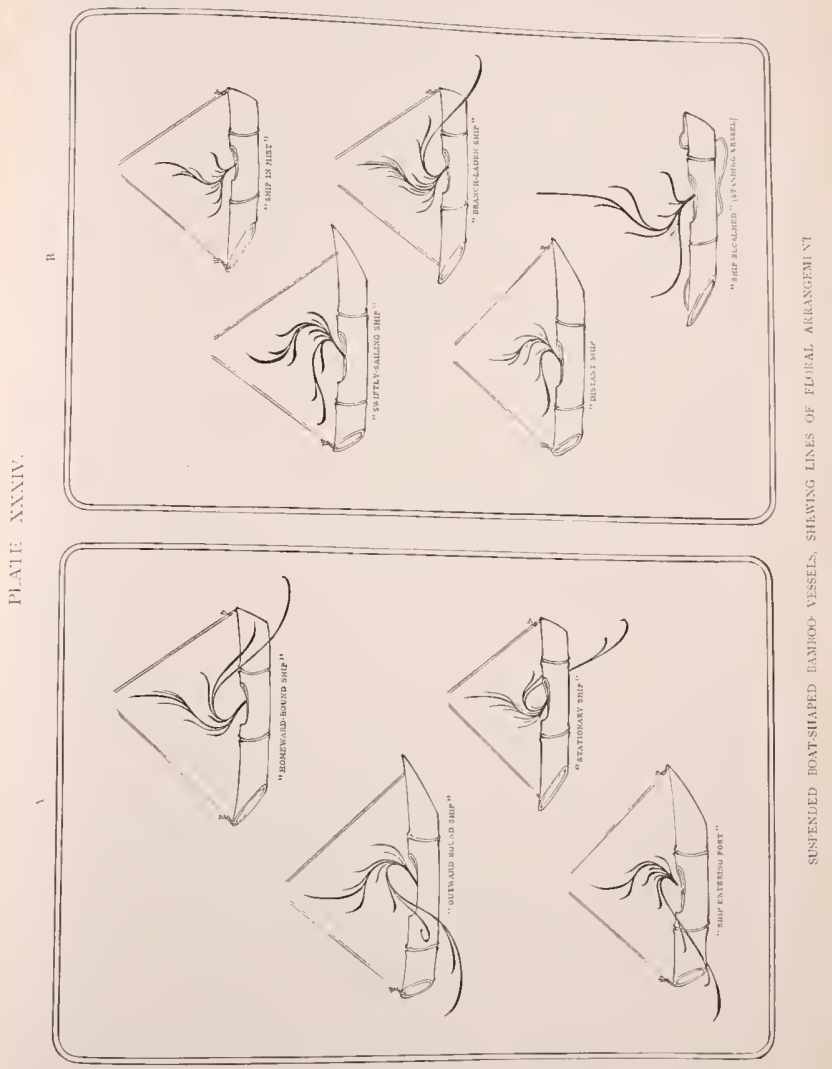


as the drayon is a mythical monster belonging to all elements, the use of this fastener is not limited to any particular kind of plant.

The Torraise-fastencr consists of one or two tortoises in bronze arranged in clifferent positions.

The Hatcr-fmat-fastencr is generally a metal instation of a pair of mandarin ducks. It is occasionally attacherl to water plants.

The Frog-fastoncr needs no special explanation, except that, representing an amphibious animal, it may be used with both land and water plants.

The Inchor-fasterer is specially intended for use in suspended boat-shaped ressels. It is incorrect to fix it in a vase representing a stationary ship, as in such a case the anchor would not be visible.

The lisif-fastener is a metal knife or dirk such as is worn in the wooten slecath of a Japanese sword, and ow's its original use as a flower fastener to a lloral arrangement once hastily extemporized by a famous artist named Orihe, in which, having no other fastener at hand, he ured his knife for the purpose. The Scissors-fostcure :

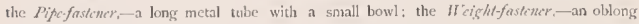
metal paperwcight the Chanfanterner,-a short chain disposed in a bunch; and the Kitlleslant-fastener, - a small iron ring and triposl used for supporting the kettle over the charcoal brazier:-these also are all occasionally employed. The principal of the above fasteners are illustrated in Plates XXXV. and XXXVt.

A special kincl of fastener, called by the Japanese Fokago, needs separate notice. The native name fakago refers to the long sansage-shaped bays of bamboo basket-work which are filled with howlders and laid in fascines at the sides of rivers to break the current and protect the banks. They are a common feature in rivur scenery and have therufore come to be initated in flower arrangements intended to be suggestive of the presence of water. The Futhagofastimers are long cylindrical baskets with closed and rounded ends which are laid in shallow basins together with ornamental stones or rocks, and besides being decorative they serve to hold the stems of the plants artificially arranged within. An illustration of their use may be seen in Fig. 33. where two are shown combined with the Serria japonica, the whole being intended to represent a view of the river Tama near which these flowers abound. 
The inverted bronec leell suspended by a chain is anoth's vencl occasonally used ler holding arrangements of wistaria Howers. Isee Fig. (6.)

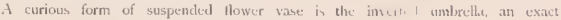
imitation in bronze of a Japanese umbrella. (Sec Fig. 14.)

A large sea-sle il lang by a single cord forms a favourite receptacle for very imple flower arrangements.

\section{FLOWER CHARIOTS.}

13r.Jonging strictly spatking to the class of standing vesucls, but sufficiently striking and important to require special notice, is the Flswer-eltariof, which ligeres so often in

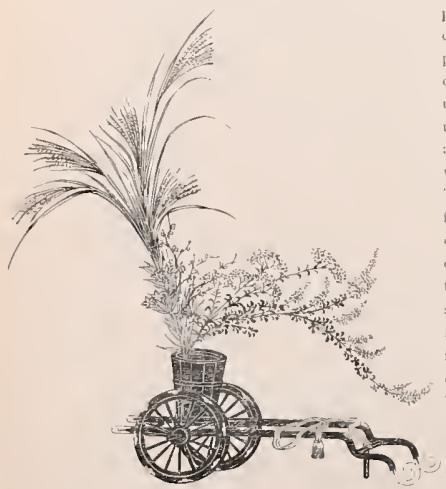

F14. 15. pictures on painteal sereens ant other decorative objects. It certain festivids and processions it alpears that large tubs full of richly atranged flowers were drawn inon wheclect chariots haxdsomely ornimented. The idea was adopted for flower arrangements placed in very large recesses, where great size and display were required. The lengtly of the Flowers-chariot in four feet six inclees from the back to the end of the shafts; the wheels are alout eighteen inches diameter, and the flower tub which the chariot carries, is about sixteen inches bigh, lioth velicle and nower tub are lacquered black :uncl furnished with silver fittings, The flower compositions are made very full and high. The Floner-eharive is illustrated in $1 \% \mathrm{~g}, 15$.

I sumewhat similar llower receptacle called the IIater-arryingr-cart is also employed. This vehicle is at llat truck 

For arrangensents in these shallow vessels there are a number of fincy favteners in common $11 \mathrm{se}$ which are in many cases merely ornamental, the hilklen cuntrivances junt described, buricd below the sand or pebbles, doing the reat duty of holdine the stems in position. The principal of these ornamental farteners are as fullow:-

The Horse's-bit-fasteter is an exact counterpart of a ponderous Japanese lit. Its use originated with the employment of the Horse-thb as a Hower vesscl, and to this kind of vessel its use is chiefly confined. The linked character of this fastener allows of its being folded in a varicty of ways, so as to leave hops of different size to encircle the flower stems, With the scrupulous minuteness of ctetail which characterizes the art under discussion. the foral designer has classified these different ways of using the horse's-bit, giving names to each bar. plate, and loop of iron, and inventing terms for the sarious methods of folding. The principal arrangenents are shewn in l'hete XXXVta. The use of the Horse's-bilfastier is prohibited, however, for Horal designs placed in the ornamental recess of a chamber of superior class: and if it be introduced into a flower arrangement in such important rooms, the composition must not oecupy the principal position.

The Crab-fastestey consists of a metal crab or pair of cralss, If nne crab be used, it should be disposed so as to contrast in character with the fiower arrangement. by which is meant, that if the comprosition be ligh and powerful, the crab must he placed in a low and unobtrusive position, but if the flower design be broasl and wanting in fertical strength. the crab must be raised in a climbing attitude. If a pair of crabs be used, one must be elevated and the other lowered in position, or, to adopt the quaint phraseology of the floral art, one must be male and the other fimalc. As the representation is that of a land and not a sea crab, this kind of fastener ma be used with land as well as with water plants.

The Hare-fastener is a bronze hare in miniature. It may not be affined to water plants, and is specially suitable for arrangements of wild plants and grasses, such as the lespedeza, rush, and elecampane.

The Pnir-of-carp-fastencr consists of a pair of metal fislz designed in the position of two carp sporting togrether. This fastener is, as might br supposed, only used for water plants.

The Dragonfostcrey is a metal ornament in the form of a writhing dragon, and, 
Sinn expressed by a suspended brumze bont, bearing white clirysanthemums, suggestive of a loaled ship in port.

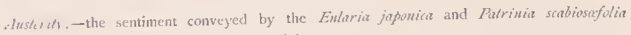
arranged together in a small bronze vase.

Quanthise. denoted by a hooked vessel in the slape of a gourd, coltuining small clargsanthemuns.

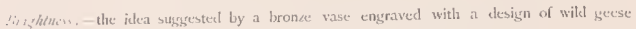
Ifying across the full moon, and holding lespedeza flowers.

Chaver, -the character expressed by a bronze vase engraved with a design representing rain, and containing a branch of maple.

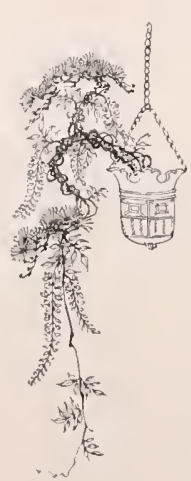

FH, 1G.

Sicurily;-denoted by sone hind of water plant, placed in a bronze vase engraved with the design of a spider's web.

Wemeralis:-the sentiment conveyesl by a branch of pine, or some other evergreen, placed in a bronse vase engraved with the representation of a crant. The crane and pine tree are both associated in Japan with the idea of vencrable old age.

The above combinations. capricious as some of them may appear, serve to illustrate the manner in which vessels and Howers are used together to express an appropriate sentiment.

Sometimes the harmonious connection between the two is baseal merely upon a resemblance in the name of both, The clematis, for example, is called $\Gamma_{i} s s^{2} n\left(T_{i} / s n-s e n\right)$ and because the word Fitsu signifies from, this flower in often placed in a rough iron vessel. (Stee Plate LIN,H)

The native name for the wistaria is Fujr, and the lyell-like ornaments lung to the eaves of temples being called 


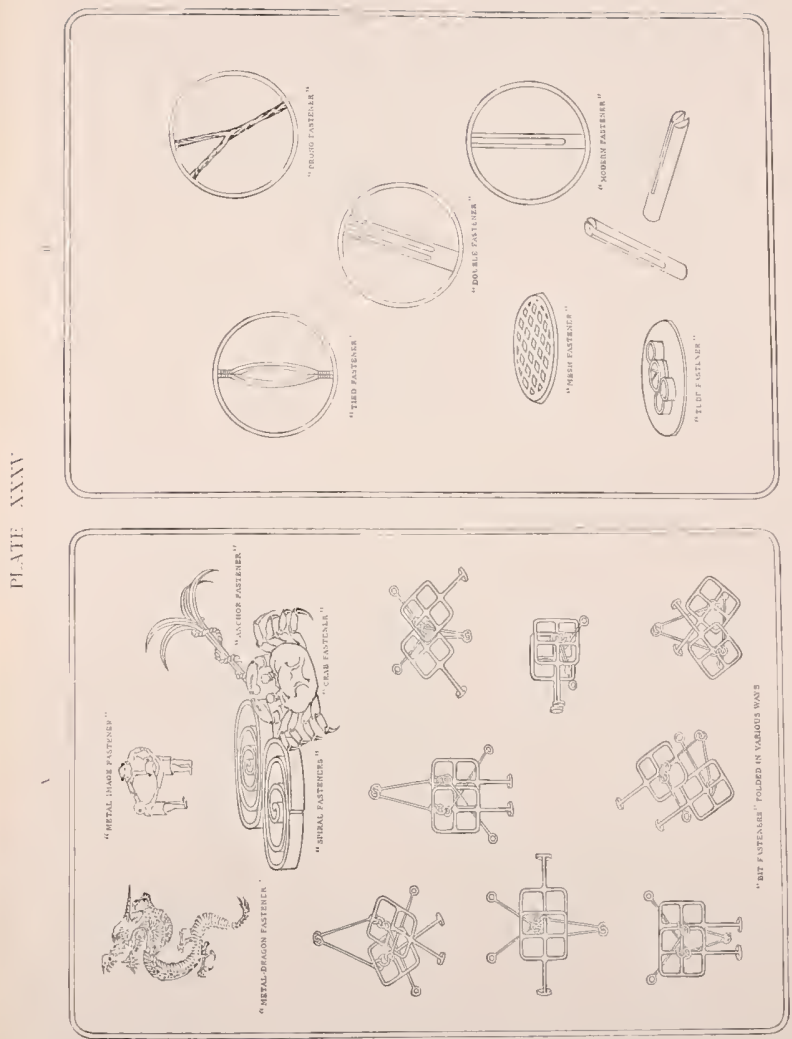


Other geit ral clirections are given as to the style of arangenicut suited to special kinds of ressels.

In llooked-arses the Aoral design shoukd suggest plants hanging over a cliff, and must te arrangel in the horiruntell triangle style. with or without a Sircamer. In vases of this class having a mouth at the side, the lines of the llowers must not eut the edgre of the aperture,

In Shathengetases of banboo with two cpenings, the upper mouth should frolel a Ire and the lower one is plam, in accordance with natural seenery in which the eree liranches occupy a higher position than the plants.

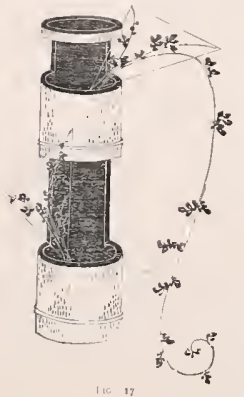

Often the same llower is used in both mouths of the sance vessel, in which case some such listinction as the following cxists:-Supposing pine branches to be arrangred in both openings, a style called the hill and valley pines is adopted, in which the top branch rejesents the pine trees on the summit. and the lower branch those at the base of the hill. The idea of distance must be: sugfoested in the former. and that of proximity in the later.

It the bamber vase have none top opening and two site apertures, a compusition called the hall. plain, and strict style is followed. To convey this idea a mountain tree is placed in the top, a land flam in the middle, and a water plant in the bottom opening.

Sometimes an arrangement exactly the reverst of this is devised in order to express the notion of a clistant landscape with a mountain lake above, fields on the lill slopes, and a forest at the base. In such a composition, the uppermost mouth of the vessel contains a water plant, a land flant is placed in the middle aperture, and at tree branch occupies the lowest position. The the branch should be arranged high to preserve the idea of a near foreground; the land plant, expressing micldle distance, may be of moderate proportions; and the witter plant at the top must be kept small and cramped in character. to suggest distance. 


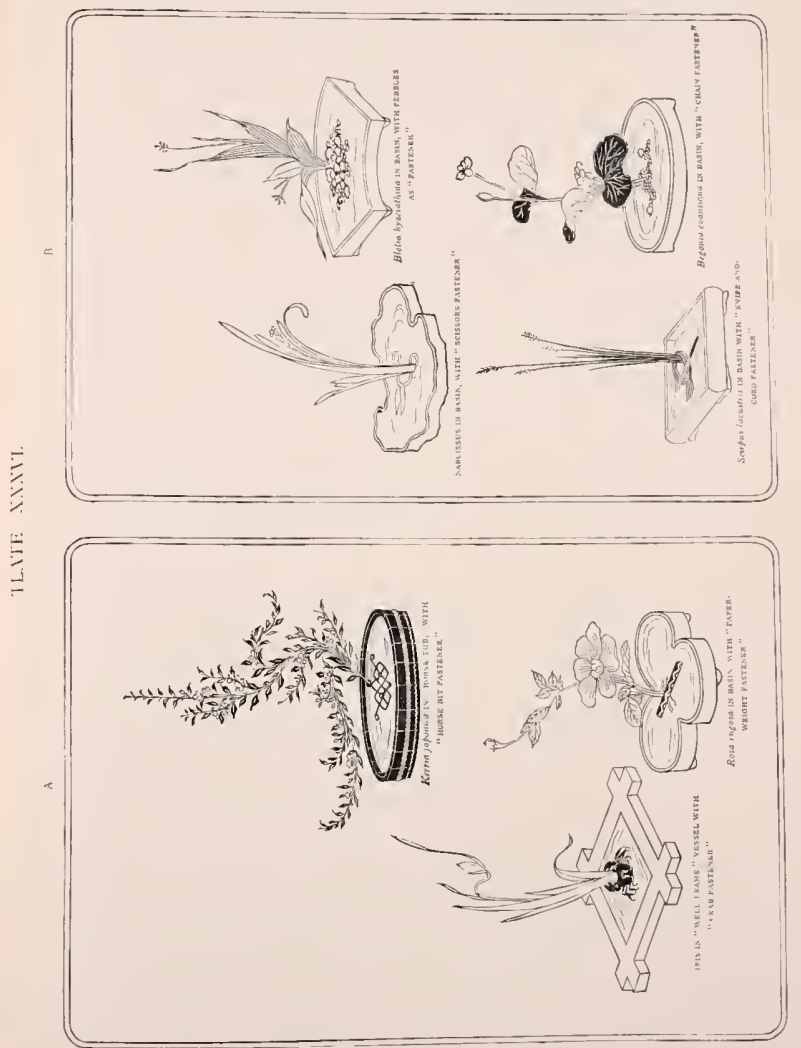


In Sand-basuss, if a tree be used, it must be supported by a Norut of some kind. Plants alone may be combined, but the composition must in stich a case be full and strong to give it an appearance of solidity. Trees or plants arranged in these vesscls are nften divided into separate groups with a space between. (Sec Plates XXXIII, and XL.1X.). If trees are used, the interval between each clump is technically called the Ialley-specte; and it should be equal to abott one-tenth of the height of the arrangement. If water plants are used in divided groups, the distance between them is regulated in the same proportion. but different terms are used to denote this spacing. Supposing such water plants to be arranged side by side and in a line, the composition is said to be in the Fish-sadimming stylc, but if the flowers are placed one in front of the other they are said to be composed in the Fish-sporting style. By a curious fancy an analogy is here drawn betwcen the relative position of the plants in such broal ressels and that of fish swimming or sporting in a lake or strean. The arrangements of trees or plants in water basins, whether in single or double groups, must be always nore towards one side of the vessel than the other, all formal and symmetrical compositions being disliket.

In Jorse-tubs the employment of trec branches is prohibited, and plants of one or two kinds must be used. With regard in spacing, the same rules apply as are given for sind-basins.

If a pair of similar vessels containing floral compositions be used side by side the flower arrangement in one should be as nearly as possilte of the same form as the ixther. reversed; but the colours of the flowers should be varied. For example, one vase my contain red and the other white flowers, with the stems or branches of both disposed in nearly similar linces, These symmetical arrangements are not, however, often resorted to,

In Flotur chariots it is usual to arrange the seven flowers of Autumn; with these are sometinxes combined other Autumn grasses, making the number up to nine or eleven difierent kinds of plants, Such elaborate combinations, which arc not generally allowed in other flower vessels, are from their ricluness technically called cmbroidery.

\section{WATER IN VASES.}

Various rules are observed as to the use of water in flower vessels. In Spring and Autumn the vase should be about nine-tenths filled with water; in early. Summer 


\section{CHOICE OF FLOWER VESSELS.}

The Japanese llower artist recognizes a distinct and inportant connection between the floral composition and the receptacle in which it is arranged, Sone points of relationship, such as that of the proportion in height or breadts between the two. and the distinction between vessels used for land plants and those suitable tor water phants, have been already mentioned. But there are other more subtle larmonics in taste and sentiment which are carefully obscrved. A flower vessel, being in itself a work of art. may possess different esthetic characteristics, such, for example, as rustic simplicity. elegance, or richness. Its shape, material, or decoration may also convey to the imagination various feelings and mental associations. Flowers, roo. possess different qualities in form, colour, or growth, and are in all countries associated with special poctic artributes. Particularly is this the case in Japan where harilly a fete or pastime exists in which Howers do not play a part, and where almost every hlossoming tree las some romantic tradition of its own. Apart from the character intrinsically betonging to the flowers themselves, their artificial treatnent in the flower art under consideration imparts to them odher claracteristics varying with the styfe of arrangenuent adopted. Thus one composition may be full and luxuriots, whilst another design made with similar Howers may be simp̧le and even austere.

Considering, then, that both flower arangements and llower vessels are works of art capabie of clistinct artistic expression, it becomes a matter of inportance that the spirit of the one should accord with that of the other. This harmony of sentiment need not necessarily be one of complete unison; on the contrary, it is often produced by a well-juclged contrast.

By way of illustrating this intimate connection between floral compositions and the vessels containing then, may be given the following ten artistic virtucs attributed to certain special combinations :-

Simplicily:-expressed by rushes and irises in a two-storey bambou vase,

Ispiration:- denoted by a vessel of decayed wood containing a climbing crecper.

Affection; - the character attributed to a bronze basin containing a pine branch entwined by a wistaria. 


\section{STONES AND ROCKS.}

In addition to the flowers, vessels, and fasteners, all of which contribute to the completion of a Japanese floral design, stones or rocks are sometimes added to arrangcments of water plants and play an important part in the compositions. These stones represent, in some cases, the large bowlders which form stepping stones over strcanis and lakelets, in other instances, they are meant to suggest islands in extensive water scenery. Again, it sometimes happens that land and water plants are used in combination in a large whallow vessel, and then the stones are disposed so as to suggest the dry bed or the banks of an adjacent river. Both white and black stones are employed, the white ones being placed near to the flowers, and the dark ones in parts of the water where there are no flowers. The flowers are arranged in front of or behind the stones and must not appear to krow out of thems

The chicf ornamental stones in a flower basin are generally distributed accorling to the favourite triple principle which is applied also to the lines of the flower composition, under the distinguishing designation of,-hazem, anth, mankind, conveying in the present ease the idea of verticality, horizontality, and intermediate form. One stone is of vertical claracter and supposed to resemble a mountain, one of flat and nearly horizontal character, and the third, which is placed between the other two, partakes of an intermediary chracter. Other stones of secondary importance are added to set off the larger stones and generally to connect the whole composition.

The use of such stanes may be seen in Fig. 25, where they are arranged in a lange basin together with wistaria flowers. In Fig. 18, on the previous page, is shown a fancy arrangement in which blocks of charcoal are employed instead of stones.

\section{FLOWER TRAYS AND STANDS.}

All standing flower vessels, with the exception of the Flowcr-baskcts, are placed upon a square tablet of polished or lacquered wood, interposed as a protection between them and the surface of the dais or shelf on which they stand. A story is related elsewhere which accounts for the absence of such tablets under flower-baskets, but a very reasonable explanation seenis to be that these baskets are not likcly to stain or 
Furem, by way partly. of a play upon the two words, the wistarid 3 , sometimes arranged in a inverted bronze bell. (See Fig, 16 ).

Certain writers go so far as to clussify flower vessels accorting to the seasons, reconmending, for Spring arrangenents, bamkon vases, bronze vases, and narrow becked 1essels: for Summer compositions, llower-baskets, bronke basins, woolen tubs, or other broal-mouthed vessels; for Autumn designs, boat-vases. and porcelain vessels, and for those of Winter. gourd-shajed vases, and narrow necked vessels. However rare and valuable a receptacle may be, it must not be uscal for holding fluwcrs unless intencled for that purpose, jars, jugs, pots, and other utensils having special uses uf their own should not be employed for floral arrangements. This rule is apparently violated in the cast: of such in.

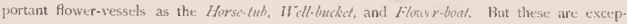
tions which custom has sanctioned, and their names have reference rather to the original models from which they are copied. In each cabc, morcover, there is a special connection in idea between these receptades and the flowers placeet in them, so thit the result has no clement of incongruity:

113 such matters, however, considerable: license is allowed to masters proficient in the art. especially in the case of floral designs for tea rooms, where the cmployment of

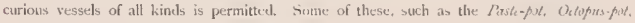
and others, are illustrated in 1'late XXX.1.

A few general directions are lait down is to the particular kind of receptectes suiterl to certain flowers.

For flowers of large-blossom, such as the peony; the Chinese hasket is profecred, the peony being considered the principal Hower of China. Moreover, these large baskets are in character well suited to show off the pronderous blossoms of this plant.

For most water plants, low hasin-like vessels, or vases with very broad mouths, are best suited, but the uarcissus requires a narrow necked vessel to show it off to advantage.

For plants of short and stumed growth, having harge leaves, it tuls shaped ressth is

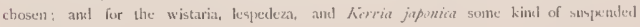
receptacle is preferred. 

In arranging flowers in tall bamboo vases which have several muths, the com. position in the upper openings should assume the from of tly. horizontal or kaning triangle, whilst that in the lowest mosth should be treated in the style of tige vertical trianglit. (See Fig. I7).

In the case of a pair of Hall-buckets, the upper vessel should have a trec and the lower one a flant. The rope should be kept sprinkleul with spray as if covered with dew. Neither of the compositions in the two IVell-bucteds must be allowed to cruss the line of the rope or chain which suspends then. Instead of the ordinury llower fasteners. IVell-buckets gencrally have a perforated lid or frane which is fitted in the top just aluove the surface of the water. When these vessels are arrangel in combination with a Will-frame, the upper bucket is suspended and the lower one rests on the edlge of the frame, and in such a case the lower vessel may show water, but the uprer one must preserve the idea of an empty bucket, and by no means must the water it holds be visible. In Spring time the floral design in the upper bucket should the the fuller of the two, but in Summer time the lower one should contain the more crowded armagement. In Autumn both compositions should be simple and quict. For arrangement in double Wicll-buckets the lower floral design shoukd be of the style used for stancling vases, and the upper one of the style employed for suspended vessels, with a lateral lean and a Sircamer: Flowers arranged in square buckets should never be placed exactly in the angles or corners of such vesects.

In the kind of double banboo vase called the Rirat of filcs, the higher tube should contain a land plant and the lower tube a water plant.

In the Bridge-ntwet-iase, a cylindrical vessel with a top mouth and an oblong side ojening, the top of the vase should have a thick stump or heavy arrangement of tree branches, and the side aperture should contain some simple plant, moulestly arranged.

In Flocer-baskicts, those with arched handles should have the Howers arranged so as to keep within the cnclosure of the handle and not cross it. In very claborate compositions this rule is sometimes violated, but in such cases the cutting or crossing must only take place on one side, and by moms in the centre of this side. The handleless baskets are generally hooked vesscts, and flower arrangements in them are lisposed as for the latter class of receptacles. 


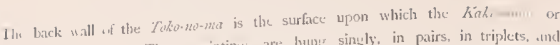
rolked uncures are chlulitet. These paintings are lang singly, in pairs, in triphets, and weasionaily in quartetts. The floral design is placed upon the this of the recess, below ind to the front of the pictures. unless a lianging arrangrenent of flowers be used, in which the it is suspended from the lintel or ecilings, or hooked to the pillar of the alcove.

It is considered important that the tloral composition should not in any way is $\mathrm{h}$ with the pictorial arrangement, eilher as tergarls position, line. subject, or sentiment. The two together mist form at harmoniuss decorative composition.

When only one wall-puinting is cahilitesl, the wase of thwers in front should be placed, as a general rule, rather to one site. if two pictures are hung, a single floral design should occupy the intertal between them, in the cirse of three pictures, two vases of tlowers should be used, ons upposite to each interval; and with four paintings, tlaree fower compositions are employed in a similar mamer. In the last case, instead of using three floral arrangements, the central space is sometimes uccupied by a statuette or incense-burner.

Vase's containing flowers are often devated upen a smell raised stanel or table. - mployed insteat of the flat boarl or tablet which is placed under most tlower vessels, One kind of table meed has a shelf below, on which a wery simple tlonal design may be arranged, and when so employed the top of the table supports same other ornament.

The proportions of the fitkeneme or hanging picture influence the disposition of the flowers on the this. In front of a Jeng painting, the fleral compersition must be kept as low as pussible, but when a short and brosel picture, called a bokmono, is displayed. the flowers may stand high and full in arragrement. The object of this rule is 10 prevent the mural paisting from being hiddea by the floral design. The sune result is often obtain. cd by placing the vase of llowers to one side of the recess, instexel of in the centre. Le in sometimes unavoidable that the flowors cover part of the picture, but under these circumsunces special care must be taken not to bide that portion bearing the stamp and signature of the artist. The centre. ends, and thssely of the ornamental roller forming the bottom lorcler of the painting must never be obstructed. When the pictorial work cantains ligures, the features of these figures must on no account be hitlden by the branches of the flower arrangement, and if, as occasionally happens, the picture is inscribed with a pocm or proverb, this writing must be exposed to vicw: 
nearly foll, and during the hottest days of Summer brim. full to overflowing, the rim of the. vessel being oiled so that the water actually seems (1) pile up above the redge. In Winter time the vase should only lo four-firhs full, and as little as seven-tentlis in tha coldest season.

When water plants and grasses are arranged in broad shallow vessels, the water forms part of the composition, and the different portions of its surface are accordingly regarded as having clifferent properties. The water nearest the flowers is supposed to he moving and life-giving. and must therefore be free from floating matter: the portion removed from the flowers is consilered stagnant and may contain floating weet of ieaves.

To add more to the fresh appearance of floral compositions it is customary to sprinkle the outsite of flower vessels with sprny, conveying the idea of dew. Metal vases should not be wetted, but nearly all pottery or porcelain vases are so treated after the flower arrangement has been made. The culadon vase is satil to form an exception to this rule, because it becomes naturally covered with moisture condensed

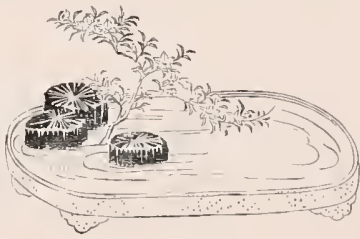

fita. in from the air.

It often happens that a vase filled with water, but without any floral composition, is used in the alcove of a chamber. Such an armagement is resorted to when there is a picture on the wall of the recess representing some flower of the season, in which case a few petals of the Hower represented in the painting may be put into the water of the vase. A ressel simply containing water and no flowers, is an appropriate ornament for the chamber at a moon-viewing party, or when a picture of the full moun is displayed, the intention being to suggest the idea of the moon reflected in a lake. Sometimes a few maple leaves are placed in the vase in order further to sustain the idea of a matural sheet of water. 
Atollin the nt utir. as they are apt to restrict the imagrm thion and detract from the

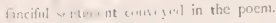

Reference th: twen mack elsewhere to the double associations from auimal and vumbable life. uscel as favourite art motives by the Japanese. Such combinations are:bamboos and s[arrows, lions and pennies, nightingales and plum blossonss, deer among maples, wikd-horses anil flowering grasses, ard many others tiat might be mentioned. A combination of flowers and picture so as to produce a connectal composition of this sort is consiktered wery desirables According to such a methorl of conzbination, a pieture of teer reguirus in front of it an arrangement of maples, a painting of horses needls wild flowers, one of lions necessitates the wse of peonies, and representations of dragons demand pine branches for the floral designs in the foreground. In the sama way with figure paintings, when the figures representeal are traclitionally associated with particular trees of flowern, such flow should if possible be employect for the flower arrangements used before them, IVhen for example, a picture, of Hotei, one of the Gouls of Fortune, is Iisplayed, bambon branches should be disjnesed in front, and before pietures of Clinese children,-1 comnon subject with Japanese painters,-coloured Dowers are appropriate.

The connection of iclea between the wall painting and the floral composition is wrs.sionally one based upon the reputation of the painter or upon some fiction witl which his name is astocined. A famous Clunese painter called 7i.Emmi, whose works are greatly valued in this country: is said to have professed a great passion for clirysanthemumis, hence, when a painting by this artist is displayed, it is customary to use chrysanthemums in the flower arsangement. Plum blossoms ar reported to have been the special f.ncy' of another great painter-Rin-lisst.-ant these flowers are therefore placed before his pictures.

This kind of combination is quite irrespective of the subject of the paintings, except in eases where other important rules wonkl be violated. If the particular flowers required are represented in the paintings, it would then be an error to use the same natural flowers in front. Such a selection woukd not only be redundant, but would tent to iletract from the excellence of the paintings. It must also be remembered that the pictorial hangings in a Japanese room are frequently changed and are not displayed contintously throughout the jear as is the custom with oil paintings in European rooms. They are quite as much an expression of the season and oceasion as the floral compositions are. The contingency, therefore, of pictures requiring flowers which are out 
damp the dais, as they contain an inner vessel which hohls the water cusd the flower

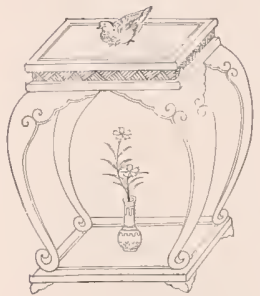

Fio, 19.

stems, Even such a simpice oljject in the vase-tray or tablet has its fixed measurement. and a few fancy forms in the shape of opern farm. circles, or segments, are not uncommon. Sonue. times this llat tray is replaced loy a smail ormamented stand or table of carved or lacquered wool. intended to raise and give more importance to small flower arrangements. As previously stated. excepting in the case of the low tub-like resseh intended to suggest water scenery and usecl for water plants, the surface of the water and the fastener holding the stems in the vase, should be a little above the eye level of the scated spectator. so as not to be visible without effort.

Some flower-stands exist which are of consitcrable height, having a bottum shelf. These are used for Incense Mectinys, in which case the top of the table carries at ornitmental incense burner, the shelf below locing occupied by a very simple flower arrangement. (See 17ig. 19). Examples of different trays and stands we slsewn in Phate XXXI.u. 
Foral designs should occupy the dais of the Tok0-no-1na, but for small and sccondary arrangements the Chigai-dana or Irrgutar Skctics are often enployed. As already explained in the case of the three operings of a high triple-mouthed vase, so for this ticr of three shelves, triple arrangenents of flowers sliould follow the natural distribution of growtli observable in real scenery. For the top shelf, thick and noss-covered tree branclies are chosen, for the niddle shelf, young tre branches or land plants are selected, and for the lowest shclf, a composition with water flants should be employed. If $1 \mathrm{rC}$ branches be placed on the middle shelf, then the lowest shelf may have land flants instead of water plants. In the same recess, and in combination with the shelves, there is occasionally constructed an ornamental cupboard about eighteen inches or two feet in height. The paper slides of such cupboards are often painted with flower designs, ancl, in this case, care must be taken not to use natural flowers of a similar kind in vases placed on the adjacent shelves.

Strict rules are established as to the exact position in the Toko-no-ma, or principal recess, to be given to suspended vessels. First, with regard to Roat-shaped vessels, it is stated that in ancient times they were hung at a distance of about three feet fron the cciling or lintel, and exactly in the centre of the recess. In later tinses it became customary, however, to suspend then, at a distance equal to about a quarter of the span of the recess, from the corner pillar, which is always on the guests' side of the room, being that nearust the light. If, as is not uncommon, two Boats are suspended together. the upper one should be about sixteen inches below the lintel, and the lower one about the same distance above the floor of the recess; but this lieight is sometimes clanged in oreler to suit the wall picture.

The direction given to the prows of such vessels, so as to suggest different ideas of motion, has been already explained. Boats are in some cases liung to the under sides of ornamental shelves or cupboards.

IVIl-brecticls are not considered suitable for the recesses of important roons, unless they happen to be a gift from a superior, in which case they may be given the place of honour; they arc, however, frequently resorted to in second cliss rooms. When one bucket only is used, it is placed standing upon the floor of the recess, raised about fifteen inches from the surface by a stand of sone sort, and removed about elcven inclues fron the corner pillar. Square buckets nust be placed angle-wise, In the case of two suspended buckets, supposing the height from floor to lintel of the recess to be divided into three parts, the top of the lower bueket should be one third, and the top of the upper bucket two-thirds, of this height, from the dais. The botton of the lower bucker 
thus becomes about eighteen inches from the floor of the recess, and is supported upon a small tablc, or on the edge of a stand representing a wcll-framc. The position of the wellpulley will be about one third of the span of the recess from the corner pillar, and the rope of the lower bucket being inclined and tort, it will be brought well to the side of the recess. If, as is usually the case, Hat-sided buckets are used, one will have its sides paraltel to the wall, and the other will present its angle to the front. Occasionally the above mentioned proportion as to lieight, is violated, and the lower bucket is placed imunediately upon the floor, with the interposition only of a board. A style exists called the Mfossy. spring.buckets in which this board is sprinkled with stones and noss. Sometimes a slab of decayed wood, or at irregular row of bamboo tubes resembling a japanese drain-board, are used under the lower bucket. The Pilat.unckicts, consisting of two standing buckets, one supported on the edge of the other, are placed upon a flat drain-board immediatcly on the floor of the recess, and neat to the corner pillar. One of these should be parallel to the wall and the other placed diagonally.

Hitherto flower compositions have been considered with reference to their disposition in the permanent recesses of chambers, either in the Fukwno-ma, or on the fixed shelves of the Chigai-dana, Other fancy arrangements, having no connection with the chamber recesses, also exist, though they are rare. Anlong such may be mentioned the arrangement of floral designs upon Cabiucts, and Flower-horses.

The Flower-horse is so named because it is made in imitation of the ornamental clothes-horse or frane which is used in Japanese slecping apartments, cither for lranging garments upon, or for carrying large strips of ricls curtaining serving as screens. The construction of such Flocter-hurse's consists of two vertical and two horizontal bars of lacquered wood. framed torgether with the: top bar projecting at the ends, the bottom bat being

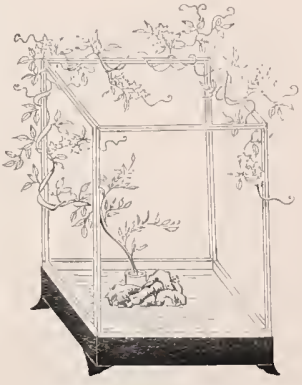

F1, 21. steadied and supported upon short cross-pieces. Such frames measure about live fact sqquare. In the example given in Plate XXXVIL. vessels of different kinds containing a 
variety of floral arrangements are liung to the sile posts and cross-piece. From the contre is suspendet a crescent-shaped bronze vessel containing an arrangenrent of small thrysanthemums and a trailing vine: from the right hand pillar are hung a cylindrical basnboo vase, with side moutls containing a drooping atrangement of Parrinia scabioscfolia, and, below, a slobular basket with a composition of carnations combincd with the branch of sume tree: and to the left hand pillat are fastened a hooked bronze vase with Paporate Theas, and, lelow, a horia-hispet bamboo vase containing a double arrangement of Platycuden grandiftermu with barley.

There exist also fancy kinds of flower-stands, made of the Flower-lwes' combined with shelves and cabincls, and on which standing. hooked, and suspended llower compositions are arranged.

Filuarerabiuets, in their simplest form. consist of two small shelves of different heights convected by vertical and horizontal framing, lacquered black, and ornamented with metal. For the upper shelf of such cabinets a drooping floral design is generally adopted, and for the lower shelf a standing arrangement. In the example shewn in Plate XXXVII. the top shelf supports a bronze vase with trailing ivy, and the lower shelf a porcelain vase containing asters. The same illustration shows a fancy cabinet called the Thurchet-kiosk. It consist of a bottom board, with raised shelf stpported on bamboo posts, and covered by a rustic thatch-roof. The shelf carries a bamboo vase from which springs a creeqer, arranged to pass over the roof and hang down the side as a Streanier.

Other articies of furniture are sometimes pressed into service for the display of flowers. An cxample exists of a triple gong-irame, with one upper and two lower nenings, from the cross bars of which cylinclsical bamboo vases containing different hinds of peach blossoms are suspendel.

A great fincy prevails for collecting and displaying together different kinds of blossoms of etrtain favourite trees and plants. The use of distinct varieties of the same blossom in one arrangement is opposed to the somewhat austere rules of the art of floral composition. but such specimens are sometimes displayed in separate vessels arranged on stumels. The regent Hideyoshi is suid to have devised an arrangement of five haskets of green bamboo disposed on the two shelves of a Floutr. Siznd, and containing different sarieties of the cherry blossom. Such arrangements, however, belong more to gartening than to chamber decoration. 


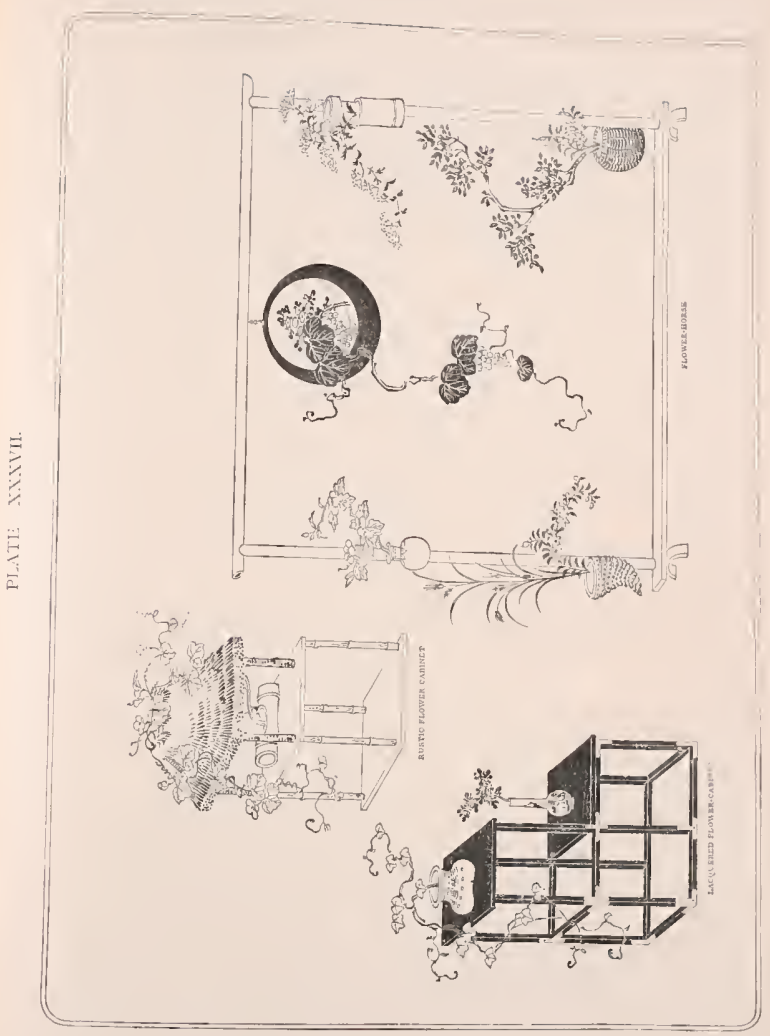



Some writer gro so far as to say that the floral design in at chutriber shuld have a contrast in style with that of the dijoining sarelen. This fancy is better apprecisted if it be renembered that during a great part of the year the outer wallis of the Japanese housc. which consist alnost entircly of paper slicles, are thrown completely open. If there be a landseape garden adjoining, consisting of lukes and lills, the flural arrangenent in the room should by preference partshe of a mextland character; but if the garden be level and waterless, then water plants or mountain trees should be selected for the flower decorations of the chamber interior. 



\section{CEREMONIAL AND ETIQUETTE.}

HE Art of arranging Howers in Jajan is essentially a polite accomplislument, and is 2 . (' governed by important rules and restrictions as regards etiquette and ceremonial. It if is presuned that all floral designs are made mainly with the object of giving pleasure

th visitors; and on certain ceremonial occasions they are actually intencled to convey a silent compliment to the principal guest. Receptions given in roons where flowers are arranged, often partake of the chanacter of Flower .3retings, the guests in turn inspecting and admiring the lrost's foral desigu, or being called upon by him to make compositions of their own.

In attending such a reception, the visitor should leave his fan in the ante-chamber and, approaching within about three fert of the recess, seat himself in the old ceremonial attitude. with his knees bent and the body resting back on the heels. One hand should be placed on the knees, while the other respectfully touches the mats in front, the body being slightly bent forward. It must be remenbered that there is always a supposed connection between the pictures which adorn the wall surface of the recess, and the floral arrangenent standing or hanging in front. The gucst should, therefore, first regard the Kakennono or picture, and if, as is often the case, there are thrce of these, he should examine first the central, then the left hand, and lastly the right laand one. Having thus bestowed his admiration upon the background of the scene, he may slide a little closer and inspect the floral composition in the foreground. In doing so he shoukl first observe the central line of the flower arrangement, and then

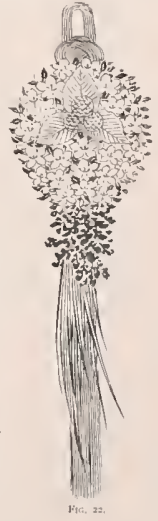
gradually exanine left and right, and from top to botton of 
the composition : lastly: with a word of apology for so doing. he miay inspect the manner in which the stems are hekl at the hotton. this being one of the most difficult parts of the techniqus. It is consideral impolite to put the face belind the branclies and peer too closely into the flowers. After such inspection the guest slides a little back and regards the whole composition from a respectul distance, using sonuc suitable expressions of admiration. Rules of etiqucttc actually go so far as to give the exact expressions to be $\mathrm{cm}$. ployed in admiring different designs; for it is considered bad raste to apply indiscrinjinte. ly evaggerated terms of praise not in keeping with the elaracter of the particular Howers. When inspecting hanging floral arrangements the seated atcitude shonld be clanged for. standing and stooping posttite.

A visitor is often invited to make an extemporary arrangement of flowers, for which purpose he is presented will certain suitable flower stems, or blossom-clad branches, and all the neccssury ntensils and implements. On such occasions the host rnust frovide
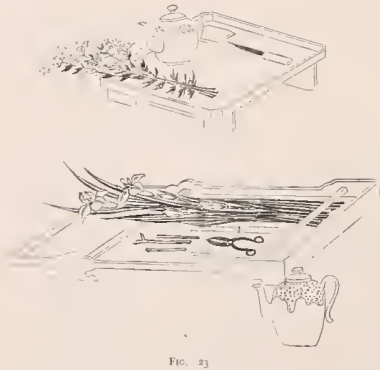

a vase, thee quarters filled with water, which should be placed in the eentre of the recess, uрол it tray or table spread with a shert of paper. In addition to this, a flower tray with two or threc kinds of eut flowers, just as gathered with withered leaves and dead twigs left intuct, a pait of scissors, a knile, a small sawv, and a folded flower-cloth or duster, must be placed on the dais to the left of the rase, or in some convenient position on the lltor of the chamber. The length, width, and manner of folling the flower-cloth. are all prescribed. Near to the above utensils anil

implements must be placed a jug full of water, and several forked twigs suitable for Flemer-fosteners. These various tools and utensils are illustrated in Fig. 23. Others. such as a hammer, and plane, are sometimes employed.

After asking a guest to arrange flowers, the host slould commence to roll up? 


\section{PLATE XXXVIII}

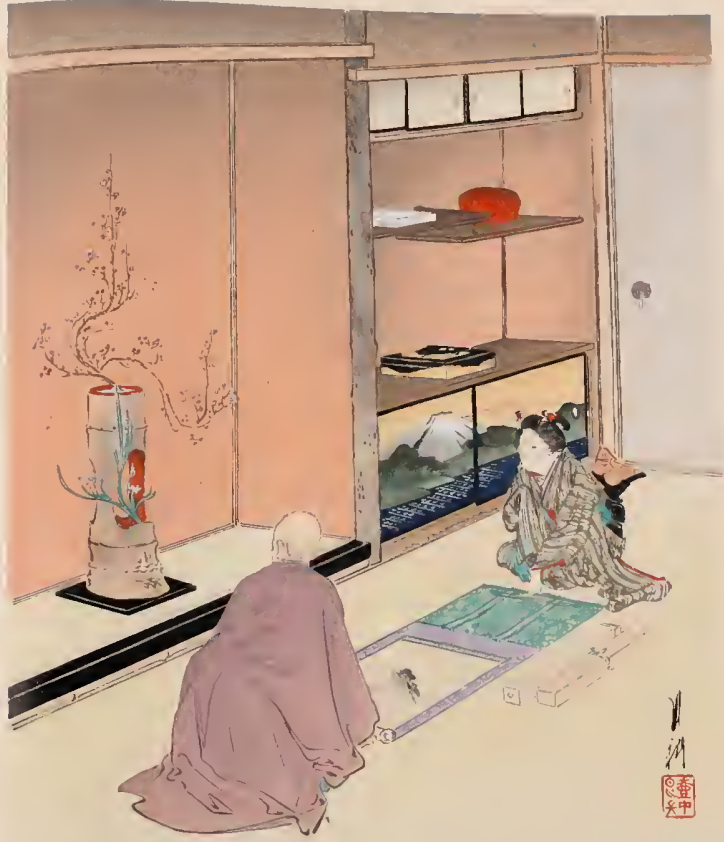




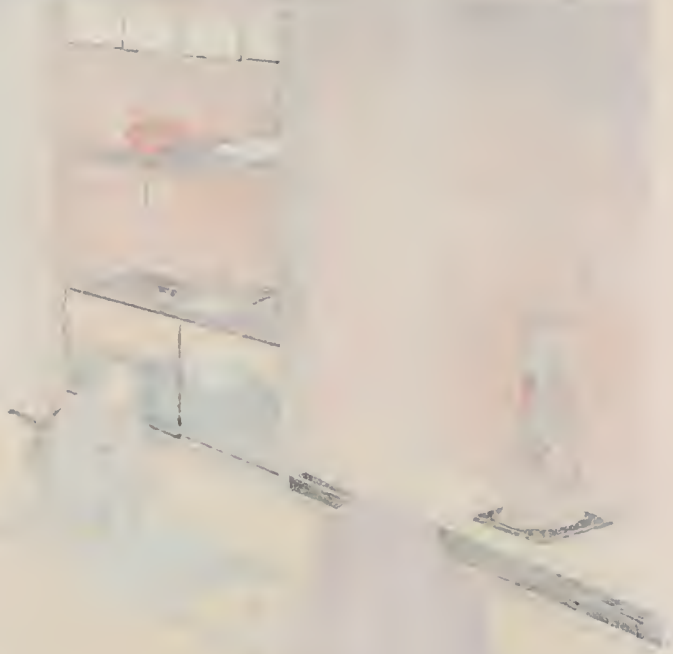




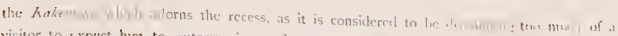
risitor to cxpect hom to extenapories a fower arrangeneat in harmen with the freture which happens to decorate the recess at the time. The gurst may, however, frevent the paintiog from being removed, thereby tacitly unclertaking to make his llower composition accord with it. Should the master of the house produce a very rare ant valuable wessel for holding the floral arrangement, it is polite for the guest to make objections, pleading want of sufficient skill to do justice to so precious a recepticle. If prosed, hourever, he must attempt a simple and unassuming arrangenent of flowers, so as not to dwetract from the merit of the vessel itself. Should the host produce in insufficient quantity of eme Plowers, the gucst must make the best of then and on no account isk for mort.

The visitor who is about to make a floral composition approaches the recess in which the flower vase is placed, and seats himself sicleway's towards it, facing the lisht, which in chambers of a superior kint is on the left side. He then fixes the flowers as quickly as possible, clanging his prosition in order to regard them from a point immediately litcing the recess. and altering and correcting them from this prsition. If the arrangenunt is intended to bave a connection of idea with the hanging picture, it is placed fronting the elge of this picture, on the side tacareat the light: but if no connection is intended, then it may be disposed centrally: Uncler no circumstances nust ux: lloral design project beyonsl the corner pillar of th. recess. Having completed thr. composition. the designer shoulcl, as a matter of conupliment, ask his entertiner to fill up, the vase witls water, and if dis request be declined, he may then replenish it hintself; he should not, however, press the host on this point. because correct jwdginent as to the amount of water suited for different arrangements requires considerable knowledge of the fower art, and it is possible that the host may decline on account of ignorance. When the water is filled in, the stand of the Mower vase is wiped, and the different implements, with the exception of the scissors, are all put on the tray, and placed near the serving entrance, or right hand side of the chamber. The scissors are purposely. left near the flowers as a silent and modest invitation to the master of the housc to correct funles. The host, brings the dust-pan and brush, sweeps up any fallon leaves or litter, and removes the tray of tools.

If the hanging picture has been renoved during the arminging of the fowers, the guest must now re-hang it, and see that the floral composition is placel so as not to clash with it in any way, When the whole arrangement is completed, the host and any other visitors present, who have neanwbile remaines in an adjoining room, enter, and. approaching in turn the Tokv-no-nan or recess in which the flowers are placel, sahte and 
inspect in the manner previously described. The master of the house inturally confines his admiration to the floral arrangement, but the guests slould also find compliments for the receptacle in which it is placed. After the Flower Meeting is concluded and the visitors retire, the gucst who has arranged the flowers should remove them from the vase, placing them upon the board on which the vase stands, or on the wash-basin of the adjoining verandah: unless he is specially requested to let lis work remain. it is considered presumptuous for him to quit without destroying the evidence of his skill (or clumsiness). This rule does not apply to visitors of very superior rank, who may be considered to show honour to their host by loaving their designs intact, however indifferent they may be.

Politeness during such mectings is considered so important that. if a rule happens to be viohted through ignorance or incapacity on the part of the entertainer, the guest must try his best uader the circumstances, and must do nothing to call attention to the error. As an exanple, may be given the instance of a host producing scented flowers for a visitor to arrange at an Inecuse Mectius. which is an occasion on which flowers with perfume are probibited, The guest must in such a case tuse the flowers, removing the rull-blown blossoms, ancl making an arrangement of buts alone in as simple and unassuming a manner as possible.

In giving presents of cut flowers for the purpose of Hower arrangements, they must not be trimmed, or they will look as if they had been previously used. The sender must howcver, in selecting thein, consider how they are capable of combination into a floral composition, and must include stems and branches suitable for the accessory parts of the design. Such flowers should have the bottom of their stems placed in fancy paper wrappers. The form of paper wrapper suited to trec branches and that usect for flants cliffers slightly. When flowers are offered as presents, the number of buts should exceed that of the open flowers, and they should be accompanied with withered or worm.

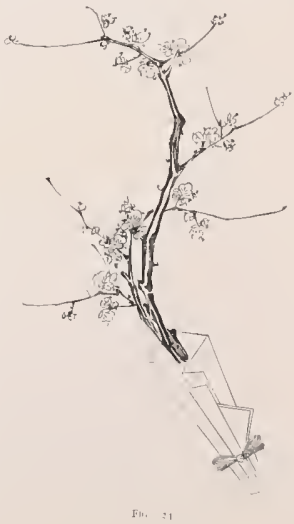


PLATE NXXIX.

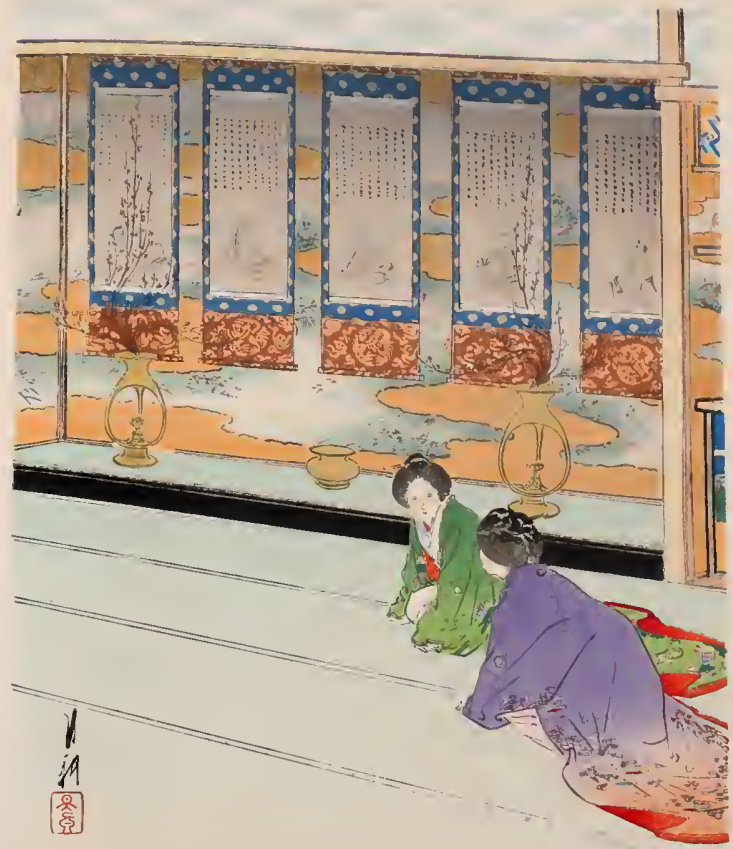

JAPANESE INTERIOR. ARRANGEMENT OF SPRING FLOWERS. 



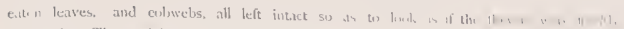

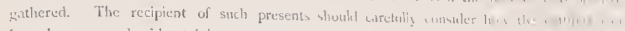

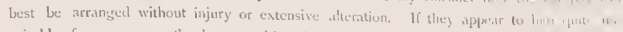

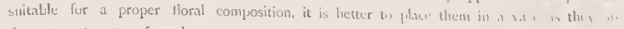

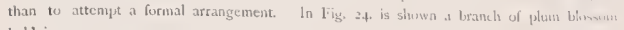
held in a paper wrapper,

Presents of flowers are sumetimes mate to superiors, in a hinal of haml-bulart, which shouk be of white wond and quite new Sewral sorts of flowers we wenerwly placed in such vesscts, which are furnislied with lids perforatel with lime or might supnar. holus for the purpose. When such a prement in reccivet. it may be put its one of the shelves of a recess in a reception room, an emply vase being placed on the dais.

In a previous part of this work tlowers comsidered specinlly felicitous during particular months have been enumeratect. It remains, hinwever. to consider, unkler the locat of Ceremonial, certain floral arrangenents which are fixeul for import unt festivals. Of the numerous fete-days celebrated in Japan, may be mentioned first that of the New

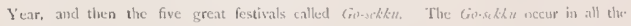
months of the year of old number. with the exception of the rescesth munth, shel, m four cases out of the five. fall on the day corresponaling to the number of the month.

\section{FLOWERS FOR THE NEWV YEAR.}

For the floral decurations of the New Year it is customiry to whopt a cmmlunet

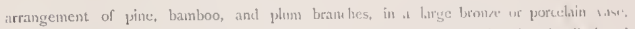
These forocrs are, however, sometimes $115 \mathrm{se}$ separately, in whit case the finc in displayed on the first, the bamboo on the second, and the plun on the thict day of the jour. In some cascs it vase of green bamboo, with twigs and lestrus left on. in thral to lwohl branches of pine and plum trees, the floral trial being formet by inclulins the ware it. self. The willow is a favourite tree for use in hangings complovitions at this seasen : ant

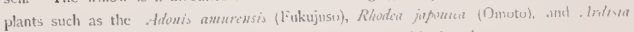
japonica (Yabukoji), are often emplojed in combination with the plam. 
FIOWERS FWR THE "FIVE FESTITALS.

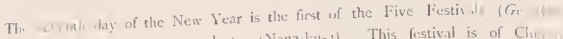

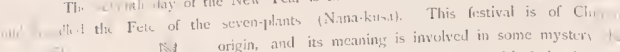

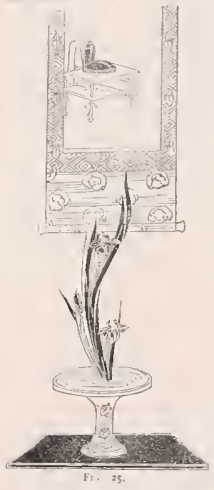

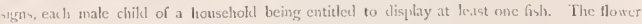
most honoured on this occision is the flay or iris. A kind of early chrysinthemum, and particular sort of bamboo, called the Mosiochikit, are also used.

The next festival falls on the seventh day of the seventh month and is called the Fete of Tauralala. It is the celebration of the meeting of two planets, and is supposed to be in day of very good omen. Stips of paper inscribed with poems atud Iraves of laubloo are humg up as ernaments on the nocasion, and a general fersting takes flate. The llowers uned for compositions on this holichy should be: Fitgenstime gratadiforam (Kikio), bamboo, and . Anthrasos arguens (Katukny.1), arranged together, or 
PLATE XI.

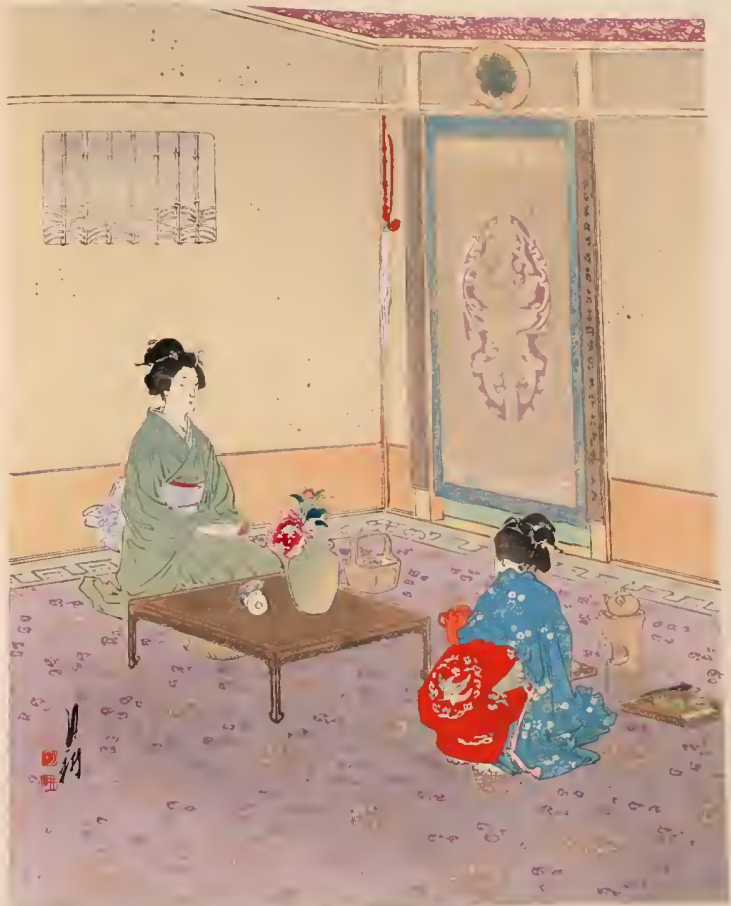




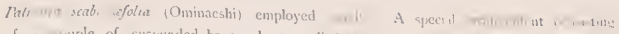

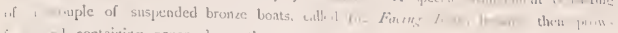

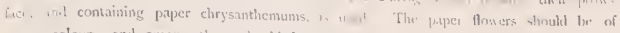

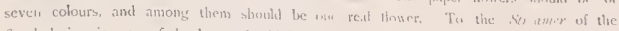

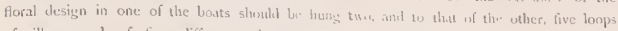

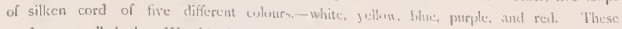

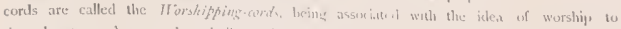

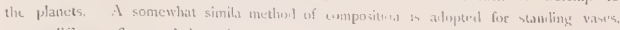
seven different flower: beines placesl in three lessels The central vibe contains three

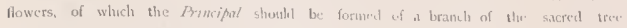

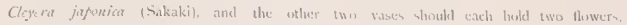
The floral designs in all three vises must have there stems bouml with the Hinstint fing-cont.

The last of the Five festicals accurs on the ninth lay uf the ninth month. unt is called the fete of clirysanthemums. Its origgith is Clintese. and it is cunnucted

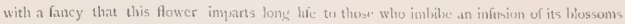
in wine. For this occasion, as the mame of the festival implies. the chrystanthemum is invariably used for floral arrangements. Flowers of fwe cwhour ate employal for first-

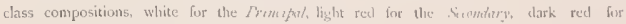

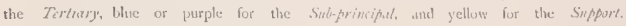
Sometines a design made with tlowers of one colour is fancilully called the firn. when composition. the green of the leaves and the blatk of the brone vare lachis

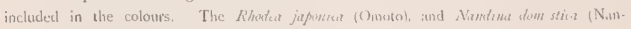
ten), are also sometimes clisphyed on this occasion.

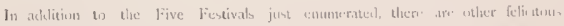

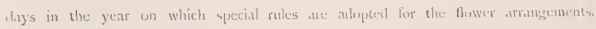

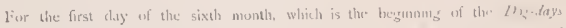

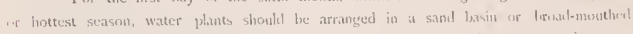

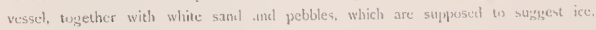

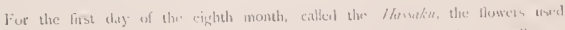

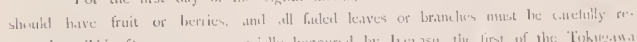

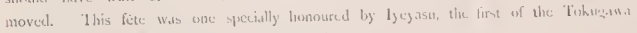
sloguns. to celebrate his cotrance into Yedo. 
On the cughth diy' of the eighth month white flowers should be displayed.

For the harvest festival of the fifteenth day of the eighth month. Howers of the veasun should be employed, with the addition of a sheaf of the ripe rice-phunt. in cetebration of the harvest.

The above special rules, though hardly applicable to any European adaptation of the art under consideration, are interesting as showing low a certain hammony of jdea should always be sought Letween the florat design and the special occasion celebrated. The following are cerenonies which may oceur at any period of the year, with genenal rules for their appropriate flower arrangensents.

\section{FLOVERS USEU AT EETROTHALS.}

On this oxcasion visits take place ard jresents are interchanged. The flower or Howers specially felicitous for the particular season, in accordance with the list previonsly given, must be used. The iris is considered specially suitud for this purpase. unless out of season, Sume people, however, object to the use of llowers of purple colour.

\section{FLOWERS FOR WEDDING FESTIVITIES.}

With regard to flowers arranged for weddings, it must be remembered that, umongst colours, red is regarded as malc and whitu as femals. Henee in the case of a son-in-law being atopted into the fanily of his bride, the bridegrooin being $\mathrm{r}^{2}-$ gardecl as the guest of the occasion, the Princifal line of the froral design shoulel be red, whilst the Sufporting line is of the femate colour-whitu. Un the otter hand. when a bride is adopted into the family of her husband, she being considered the gutse of the occasion, the white colour has the central position in the arrangement. In both cises the stems of the flowers used must be closed, and firnsly conjected at the tase with coloured cords, called M/isuhiki, to signify union. The banboo and pine. being always in season aud spechally felicitons, are considered well suited for wectling ceremonies. They should be placed separately in a pair of similar vases, the pine lowards the guest's side of the chamber, and the bamboo towards the hosis side. Jurple llowers, willow branches, and ocher drooping plants are prohibited for 


\section{PILATE XII.}

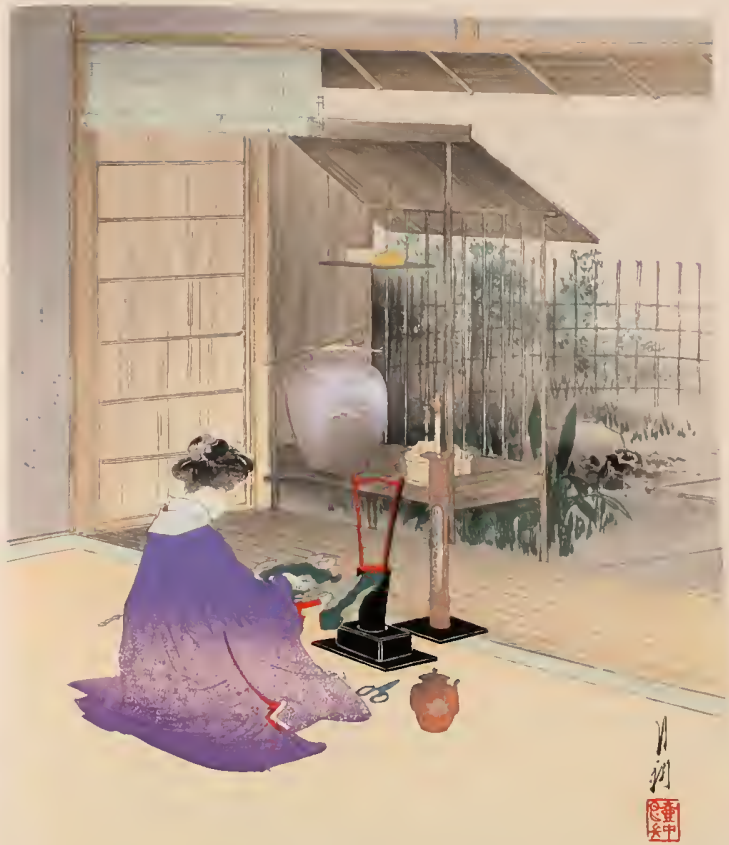

JAPANESE INTERIOR LADY ARRANGING LOTUS FLOWLRS. 


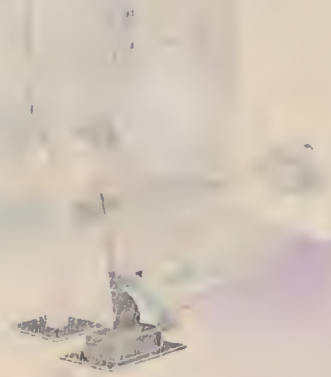

I 
weddings, as also are all floral compositions placed in suspended vessels. 1'urple is consitlered expressive of mourning. and all lonse drooping arrangements imply disunion and inconstancy

\section{FLOWERS IOR COMING OF AGE CELFBR.ITIONS.}

There were formerly two coming of age celebrations for males one on the occasion of the boy first assuming the hakama or cerenonial trouscrs, and the second when his long locks of hair were cut off and the youth assumed the che worn by adults. For both of the above festivals, flower arrangements were required to be firn and wigorous, with a large proportion of buds axd young branches, Faded branches and fill blown flowers were prohibited.

\section{FLOWERS FOR PROMIOTIONS IN RINK.}

Flowers used on the accasion of honomathe promotions sloult hine bukls below and open flowers above, to signify ascent in rank, and the use of withered leaves or overblown flowers must be carefully awoided.

\section{ILOWERS FOR THE CEREMONY OF RELIGIOUS RETIREMENT.}

Upon reaching a ripe age it has always been the custom in Japan to seck retisement from active life, the head being shaven and a semi-retigious cerenony performed. Floral desizns used at such a celebration sloukd consist of berry-bearing plants or trees, and red flowers must not be emplojed.

\section{FLOIIERS FOR OLD AGE CELEBRATIONS}

Old moss-clad branches, flowers late in senson, and second flowerings, are suited to such occasions. Wirhered branclses and howers which quickly fade or f.ll to pieces must on no account be used.

\section{FLOWERS AT FAREWELL. GATHERINLS.}

There are certain flowers which bloom twice in the same year, and these are tcchnically colled Returhing-fleners. Though considered unsuitable for ortlinary occasions. 
hecaus oust if marmony with the season. such flowers are specially appropriate for farewell gatherung? The idea in so employing them is to express the liope for a safe? return. Similar arrangements were adopted at feasts before a hattle or campaign.

\section{FLOWERS FOR THE SICK.}

Flowers placed before sick persons should be put together in a ripid and

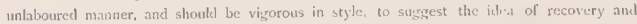
strength. At the cerenony of praying for the sick, flower compositions shoukd be full and riny, as well as bold and powerful in style. The use of the pine, Itadearpus marrophyllat. anel Rhodea jopenica should be axoided.

\section{FLOWERS BEFORE HOUSEHOLD SHRINLS.}

Eich household in Japan has generally two shrines, one dedicadest to the household gots of the old Shinto cult-the national deities of the country:- -uad the other to the spirits of decensecl relatives, which is Buddhist, For arramgements of flowers before the Shinto shrinc, a full and powerful composition is required. All ugly flowers, those of strong odour, and those having thorns are prohibited. A special branch called the fancins. branch is used behind the Principal line of the composition and pointing towards the shrine The floral design jhecd in front of a Buddhise shrine shoukl also be full and croweled, but the arrangement should not be laboured. The front of the design must face the shrine and not the spectator. A story is tolal of a Daimio who went to his ancestral mortuary elapel and arranged flowers with great skill and labour much to the aulmiration of the chict priest, who, however, pointedly asketl him whether he had made his composition with a desire for public praise or in reverence for the spirits of the deau. Unable to answer, and struck with the justice of the reproof, the noble altered the Hower arrangement to one more simple and unassuming. White flowers are sjecially suited for such designs, and in all cases, as little artificial trimming and bending as possible shoukd be resorted to, the withered leaves only being removed.

\section{FLOIIERS AT DEATH ANNIVERSARIES.}

For floral clesigns from the first to the fifieth anniversary of a death, a quiet and simple style must be adopted White and yellow flowcrs are uscel, and with then is 


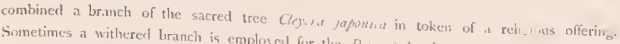
Sometimes a withered branch is emplogal for the Primesat. As such llower arrangremem. igmorant and in bul the composer must not attempt to display still, it is considereal The selection of flower buts is the them. juslgme them by the ordinary standard. preferred. The us: of crossine le be avoided, full-blown of nver-blown flowers bein: forbidelen.

On and after the fifticth anniversary, zayer arrangements and even ret flowers may be comployed, and the I'rimeipal line should be formed with an old moss-coverist branch, a lower of the season being adted as an ausiliary. Flowers of ominous nancs. such as the On-ynri (demon-lily). must on no account be used.

\section{FLOWERS AT PRAYERS FOR RAIN OR FINE IVTATHER.}

There are special rules with regard to flowers which are suited for the occasions. of prayer for rain or fine weather. As it is the east wiml which brings rain, lloral arrangements used at time of praying for cain shubld have their Prencipal line pointing from right to left, to suggest the cast wint blowing. A reverse arramenent is resortet to on occasions of prayer for line weather, when the Principal line, leaning from left to right, is makle to suggest the west wind.

\section{FLOWERS USED AT MOON VIEWWING.}

Moon viewing is at alt times a favourite fastine of the Japanese. but the great moon festival of the year is on the fifteenth day of the eighth month. The more important divellings have a special clramber with open galleries from which the sight of the moon-lit landscape cau be enjoyed. The Horal arrangenent occujies the recess of the chamber and has of course no real connection witls the outsille prospect, but in the flower composition itself the iflea of a moon-lit lanclscape is expressed. $\Lambda$ brancly of a pine trec is used, and between the Primupat and Srondary lines of the comprosition a special branch is introduced, fancifully called the .16on-shadow-branch: a hollow gap also is tormed between the foliage, bounded by a special branch called the Dividing brandh. In the composition, the idea is to sugurest both the opening through which the moon can be partially observed, and the dark branch which appears to cross its surfice. To fully appreciate the analogy one must be familiar with the scenery of Japan, and hrve seen, on a clear vight, the irregular pine trees standing out against the moon-lit heavens. 


\section{WLOWERS FOK HOUSE WARMLNGS}

The Japanesc word for red (//i) is phonetically the same as that which denotes firc, and as conflagration is the dreaded enemy of the beautiful but perishable buildings of the country, a superstitious objection exists to the use of red flowers on the occasion of such celebrations as house-warmings. Flowers the names of which contain the ominious ideograjth $\mathrm{H}$ are also prohibited. Such Howers are, the Itethenthus (Himawari). Luctinis

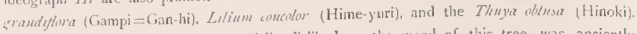
The usc of the last, (Hlinoki), is specially disliked, as the wood of this tree was anciently employed for producins fire. By a curious perversion, however, some Sclsools approve and even favour the use of Hinok, because the name is phonetically synonymous with another word hisulit meaning protection from fire.

IFor floral designs on occasions of this kind, a two-story bamboo standing-vase is suitable, having water plants arranged in both openings, the reason given for such an arrangement being that water is $\mathrm{t}_{x}$. extinguisher of fire. Another fasourite combination in Summer is that of a white inanolia branch with white irises. In Winter, suitalite flowers are scarec, and the narcissus is used in the lower opening of the vuse, with water alone in the top.

\section{FLUIVLRS AT ROETRY MEETINGS.}

A favourite pastime of the educated classes in Japan in former times, was the meeting of fricnds for the composition of verses. Sometinacs a flower of the scason formed the stbject of competitive versifation, and in this ease the Enstriu School hald that the flower chosen as a theme, if in season, shoult be displayed in a simple and quict arrangement in the clamber of meeting. Sone Schools were, however, of a contrary way of thinking, maintaining that the particular flowers stlucted as subjects for poetical composition should not be chisplayed at the mecting.

\section{FLOIVERS FOR THE TEA CEREMONIAL.}

As the chambers in which the tea coremonial is conducted are of very limited size, the flower arrangenzents must le small in scale, simple, and unaffected, 
The displaying of the floral design has its proper orter w the tha cerim.m. It is not generally placed in the recess at the same time as the hanging ficture. First, some suitable kakcmono or painting is shown, and a part of the telterthimuent takes place, after which the guests retire: the kak mant is then removed, ind the flower arrangement is prepared before their re-admission. Partly to avoid keeping the visitors waiting too long, and partly: on account of the selere simplicity of the surroundings, and the austere character of the entertainment, the Hower composition is mate as unassuning as possible. The kind of floral slesign aclopted for tea moms, differs, therefore, from the ordinary style. being less claborate and studied: it is distinguished by the name of the Thream-in-stylc. If a standing vase be enployed, the composition consists of the Prumerpal line used singly: in a hooked vase, the Secontary line alone is usecl; and for a suspented vessel, the Firtiary line is preferret. If three stcms are combined in on design, they must not be divided. but muat be hept togedfer in one line. Fowers used niz sueh occasions must be well sprinkled with spray, to look fresh and as if covered with dew. When a tea cercmony takes plice in a large room, instead of in thet orthotos minizture chamber, then both the flowers and mural picture may lo displined at one lime. If such meetings are held at night, ftoral arrangements are not employet. A houked or suspended arrangement of Howers is preferred frit toa rooms. Rid blossoms, and flowers having strong perfomes, are disliked. The following flowers are also whjected

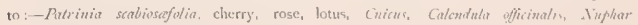

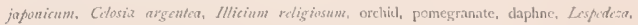
drooping lily, drooping Platyrodon, tea plant, and duthistiria arguens.

One of the objects sought in arranging flowers in a tea ronm is to arouse the admiration of the guests by the quaintness and novelty, as woll as the unassuming simplicity. of the composition. For this reasen, curious and unusual vessels art often resorted to.

\section{FLOWERS FOR INCENSE MEETINGS.}

Another polite pastime of the Japanese in former timus $w$ is that of burning different kinds of incense, and of distinguishing the scents produced: a small rnom like the tea room being generally used on such oceasions. The little bronze or porcelain incense-burner lecame the principal art object of the chamber. and was supported on a stand or low table, about one foot high, placest on the this of the recess. The height of this stand necessitated that the hanging picture should be a broarl low one, techuically 
called a Crass-pidi: (Yoko-mono). The most simple kind of floral decomation at these cntertainments was that of a single flower and leaf, or a small bunch of flowers, placcel in a tiny vase below the stand or table. (tine Fig. 19). Some theorists object to this arrangemeit because the incense-burner above contains fire, and both wind and fire are injurious on fiowers. If not uset in this manner, the flowers should be placed in a vase booked to the pillar or wall of the recess, or a suspended boat arrangement may be adopted. All scented flowers of whatever kind are prohibited at incense-burning meeting. This rute excludes such flowers as the plum. daphne, chrysanthemum, and Aconitum fischeri.

In cases where a hanging incense-hurner is suspended from the lintel of the alcove, a standing arrangement of flowers may be placed below. 


\section{MANIPLLATION}

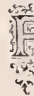

REPARATORY to the wedying of plam and tree cuttings inte iases and ...twer 3). Wriwreceptacles, certain procautions are necessary in orfler to prolong the vit.lity of the s. $y^{2}$, vegetation thus employed. For, however well designed a flower arrangenent may be. "unless it possesses a certain degree of vitality and endurance, it will specdily loue its shape, and become limp. drooping. and fadecl. It is important that unteinge from treces and plants should $\mathrm{l} r$ made at dawn or dusk, exeept in the very coldest season. If nut manipulated at ance, they should be kept in a coot damp place until required, haicl if possible upon stone or cement paving. Thry may with advantage be suspended down the hollow of a deep well, but not so as to clip into the water. Lange delicate blossems or keaves are wrapped in soft paper, and stems are loosely bound tongether with soft corel to prevent injury. After kerping in this manner for some hours, and sometimes for it whute. day, the cuttings, just before use, are laid floating in tubs or bowls of clean colel water. During the heat of summer, and at such a perioul only: this Acating in water takes place inmediately after cutting. In temperate and cool scusons, however, immedi.te immersion in cold water is found to be injurious to $\mathrm{hng}$ vitality, and productive of brittleness in the stems, which have to undergo considcrable bending durings their preparation for combining intn a flower arrangement. In the cold and frosty westler, floral cuttings are not laisl in water. but simply sprinkled a litk ancl kept in a shadly phace. The stems and branches are at sakch season very brittle and difficuit to bend without loraking, and it is usual to slowly warm and thaw them oter a charcoal hraziey, in order to make them softer and more pliant.

The two essential points to be secured with cuttings for flower arrangements, are, first-- to preserve the succulency of the stem catremitice, so that the water from the vase continues to rise into their fibres; and second,-to obtain soffness and pliability in the stems and branchus cnabling then to yield to the twisting and lending which is necessary in order to give them the required curves.

The cut prouluced hy scissors or knives in removing branches or flower atems from the parent growth is unot found to be conducive to the. long vitility of steh cuttinge, 
when wedged int, vases enntainingr water. Without professing to coplain this scientifically, it may $l x$ surmised that the surface of sucls $n$ section given to a branch or stem las a tundency to close and heal. thus choking the pores by which it is necessary that the water should rise. Some growtls are of course more hardy than others, but with many. an artificial treatment of the extremities of the cuttings, before wedging then into their receptackes, is found to considerably prolong vitality.

The stens of the iris, chrysanthemum, and sonse other plants, have their ends 1 laarred by fire to increase their succulence. In the case of the bambeo, wistaria, rush, monochoria. lichnis, and other srowths having knottexl stems, this method is found to be uscless, Charring is, moreover, never resorted to in the Summer tine. The stems of some ylants, - the peony for an cxample,-have their ends plunged in builing water, containing sulphur, or sometimes a little arsenic is used instead of sulphur. A mechod followed by some masters, is that of maslying or crusling the enls of the cuttings, using the back of the flower-scissors for the purpose: this is the manner imariably adopteal by the lkenotro school. Whether the extremitics are burnt, Liniled, or crushed, the cuttings so treatel are aferwark helel upside down, and a slsower of water poured over them for some minutes, Bintbon tulues are specially treated tin keep then green and fresh. They are prosged tlirough the centre with a squewer so as to pieree all the intermediary knots or divisions, leaving the bottom one only intact, and then water is filled into the tube: Sometimes a dilution of boiled cloves or of rice wine is added to the water as a stinulant.

The artificial curves imparted 10 branches in Japancse compositions are produced by careful bending. given with the two thumbs and fore fingers placed close together. and with a force just short of breaking. Sone stems of more yielding claracter will readily assune the required form without snapping, but the harcler and more brittle kinds are variously treated. They are sonetimes shaved with a knife at the points to be bent, or are softened with boiling water, or heated over a slow fire. A cloth or ray is wrapped round the part to be bent, to prevent splintering. If dipped in water after such forcible bending the elasticity is destroyed and the branch is prevented from returning to its original shape. To avoid ugly angles it follows thint several bends have to be make in a branch to obtain the required curve.

Means are resorted to in onder to keep large heavy blossoms in position in compositions and to prevent them drooping or folling off. Camellia flowers quickly fill. and it is found that keeping damp the junction of the blossoms with their stems by 
means of salt prevents this. W'ith peonies, large chry's, mthe it, magneli.t. indlowers,

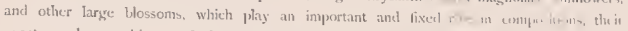
exact number, position, and direction being fixed, it is often f1...-. 1ry to resort to the artificial support of hidden bambou spikes or wires, though the ane are trickis not encouraged by the masters, for fear of being aluned, Even painting is ererctly reserted to in some. cases to preserve the apjurent freslmess of colour in flowers anul leaves, of to give. them the appearance of exther more watiful specimens. The writer has leard of red Limellia Howers in compositions being dotted with sulphor to give the apprarance of the variegated kind of blosson. The green pine needles, which quickly turn brown and lose their gloss, are often washed with a mixturs of gamagoge or sulphur and sire. The bark of tree stumps is alstz sometimes painted in a similar waly.

Nethods which are stritty spraking in violation of the principles of the art as

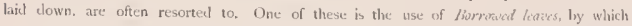
is meant leaves of one plant used to embellish the Howers of another plant pussersing somewhat similar leaves which are not in contition at the time. There must be some resemblance, however, between the real baves and the sulstituted matcrial. The knwe's of the young oak tree art in this way sometimes alled to rhodotendeon flowers, the real leaves of which wither and fall before the blossums.

In like manner Borrowed flowers are: sometimes added to handsome leaves of a different plant the Howers of which are in puos condition. Thus, to the leaves of the

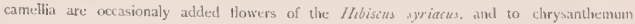
leaves, the flowers of the fmule britannica are sometimes united. The resemblinee here is between the flowers and not between the leaves of the two growths that are combined.

Lichen and moss are produced ugen loranches and stumps of tree cuttings by artificial means. For this purpose the branches are placed on a tile roof experseil to the wet, or kept on the shady side of a house in a slamps spot. Mloss is consiclerial decsirable

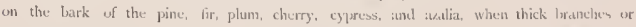
stubs are used; and lichen is considered stritable for the pine, tie, oak, maple, and willow. 



\section{PRACTIC IL F.I.IMIPLES.}

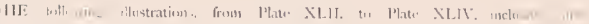

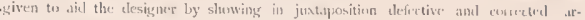
rangenents of the same floral bianches or loaves.

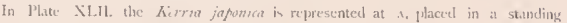
vase, with its branclies very much in the form assuned when fint gathered. The itefects of this composition are, the stiffness of the central steut, the weak and oyun appearance of the branches at the base. the rcelundancy and parallelisis of some of the smaller ypays.

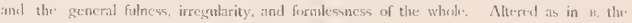
central stein of Itrmigal assumes a stronger curve, the different branclues are united all the base, the lines of the Scrudary and ferviary are improwed, and some of the smalter yorays semoved, so that the whole becomes clisposed into fixe zoverning lines.

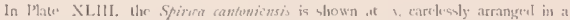

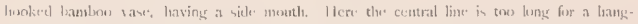

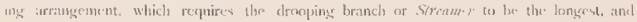
the loranches are tewo numerome and strasglings. Alecred as at 11, the franctpal is shorten.

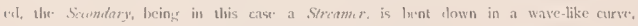

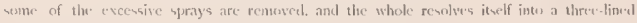
, arrangencent.

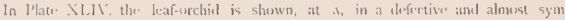
metrical atrangement. The princigal fauts of this composition, ar the straightness of the crentral leaf, anil the monotonoas manner in which the fronts and backs uf the leavere, as shown by the shading. are clisposed, Inmproved, as at $\mathrm{n}$, the stem of the centrit leaf is sracefully curved, showing its front surface with the redge curled over, and the other 
leaves are united firmily at their lanse, arranged in steps, and disposed so as to reveal alcrinately front and back surfaces in a well lulanced variety. The whole assumes the chancter of a tri-lineal design.

The special rules and tralitions which gotern the arangement of partienlar finwer will now $t_{x}$ explaincel, pratctical tamples being shown, so far as the scope of this work permits, in the aecompanying illustrations.

\section{ARRANGFITENT OF PLUM BRANCIHES.}

The phum blossom leeing the sarliest flower of the jear, is held in higls esteem for floral arrangrments. The hardiness of the phom tree, the duration of its blossom, its sweet perfume, and the austere type of its heauty; all help to make it oven a greater favouritu for Hower compasitions than its mole showy rival the eherry tree. There exist in Japan many varieties of this binssom, - ret, white, single, and douldes. Branches of red plum blossoms shothl te arminged in a fuller anel gayer manner than those of white blosson, which should be thisplayed in a quiet and open style. For Siprings arrangements a fancy presails of suggesting, by means of a pereh-like lw-nd of a beanch in the composi-

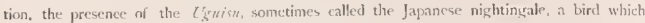
delights to make its home of song in the plum trees. Heavy and antinue standing vases are the most suitible receptacles for this flower.

The rough and irregular claageter of the branches of this tree, renclers comsiderable trimming and bemling necessary in oreke to arange them into lineal compositions, and this manipulation is assisted ly softening refactory portions in hot water. In Mats XLV. is illustrated the process of composing a tri-lineal design with small branches, rach stem being shewen both in its matural and its altered form. sicle by side with the completed design.

But the farourite kind of plum branch for chanber decoration is that of the thick, rugged, moss-covered trunk, with young branches and shouts attacherl. The fresh shoots, which grow vortically in nearly parnllel lines, are specially admired when combined with the rugged trunk, and ar: introduced under a special name. (Zwwaye), taking their appropriate: place in compositions. Arrangements or this kind vary accorcling to the class of room in which they are uscel. For important chambers a comjonstion Iraning to the right is acloptecl. and for secondary roms one leaning to the left. Such designs are said to have respect- 


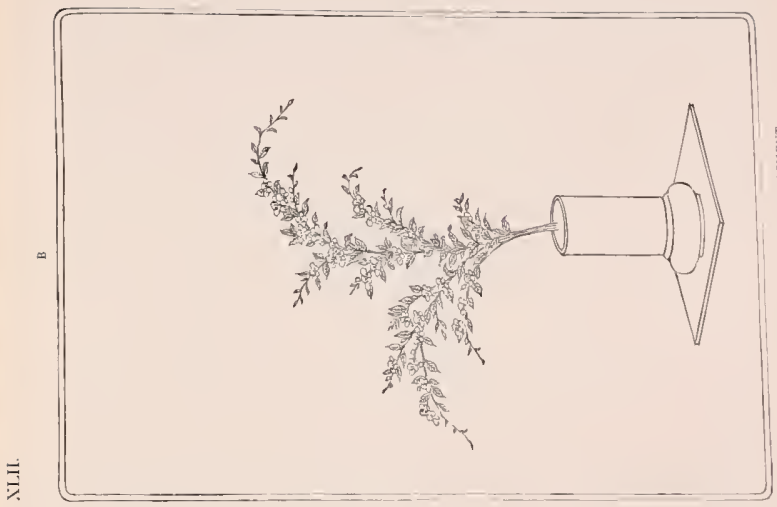

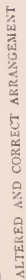

点

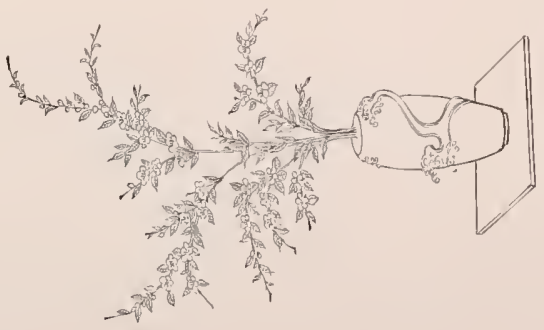

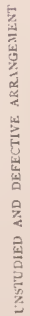





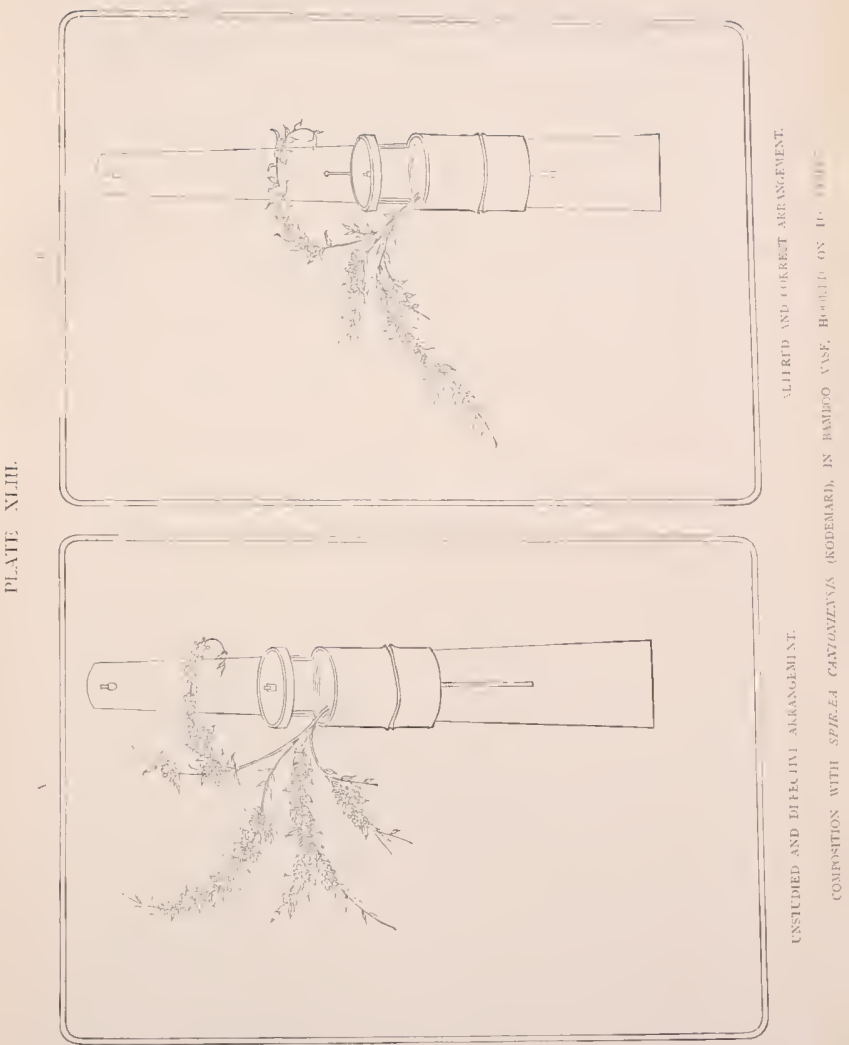







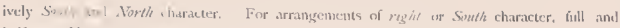
half-open blossoms predominate. 1suds being employed sparingly in the Forrary branch only.

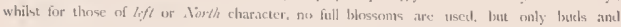
half-upen flowers, in mose quict and bare character hering given to the whole composition The shoots art, in hoth designs, sparingly intreduced, springing frem the main trunk in

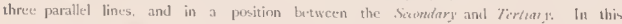
particular, the plum tree furms a privilegul exention to the genetal rule prohiliting perallitism in the disposition of stcms or Inanches.

A curious arrangement of plum branches in a large shallow basin fillect with water. ilfustrated in l'late XLIT., requires special notice. As at gemeral rule these: broal water-basins are employed only cluringe snumer, and fur the diyplay of trese or plants which grow near or in water. In the present Labs, the comugesition consists of an irregularly bent cutting of a plum sowe dicproeal in an whlong shallow vessel, in the fient ing style, with the principal branch diving though the water and reappearing bejonel. The explanation of this fanciful arrangement is that it was originally in initation of the

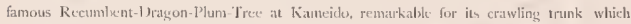
ploughed the ground in several places before re-appearing elowl with bussoms, In the artifcial composition it is consitered important that the extremity issuing from the water should rise firmly; to suggest springing firom the exrth, abd should, in fact, be lixid as if it were a branch separately helel below, though sufficiently conveying the idea of continuity of line with the original branch. Siome Schools otherwise explain the origin of this style of design. By then it is called the plum of the mountain stream, or the waterdiving plum, and its introluction as a Horal design is attributed to the asthete Solo, whe. dusing a hunting expeclition, observed a crooked old plum tree dipping in this manuer into a mountain stream, and aclopted the idsa for application in the flower art which he prat. tisecl. A tree of this kincl, measuring over a hundred feet from its poot to the extremity of its branches, is saich to have existed near Kioto. In the above arrangements, sand and gravel are mostly used for helding the phum branch in position. bot somctimes the horse's-bit fastener is employed: in which ease, for reasons already stated, the composition must not be placed on the chais of a chander of importance. In one of the illustrations of clamber interiors, (Plate XXXVIII), in shown a esmuposition with plum branches in a banboo vase containing also narcinsts liowers dxelow. The clotlings of the ligures indicates the early Japanese Spring,-the season of the plum blossom. 
The plum 1 . used in combination with land and water flant weth on the Sipu

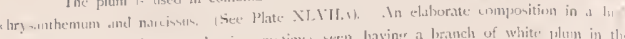

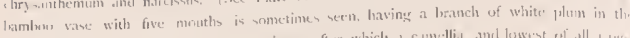

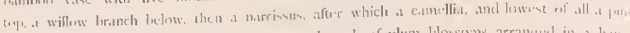

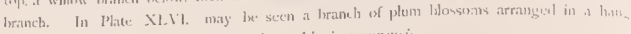
ing basket, in combination with the plint . Adouis amnrensiv.

\section{ARR.ANGEMENTS OF PINE BR.INCIILS.}

The pine is the muse impurtunt of all llowerlese trees in Japan. The hardieni an neblest of evergreens, and a constant feuture of the landseape, - whether it be mouncin in

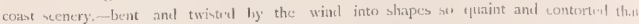
fuidfal imitation in miniature in,ty well be mintaken itus grotengue caricatures, thl vencrable tree ferms at favourite sulject for poets and artiots, amal tinets an impurtum place in floral compositions on all congratulatory occasions. Combinal with tien fhum

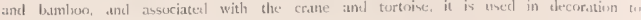
express the sentiment of trayje uld nge.

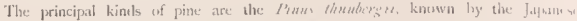

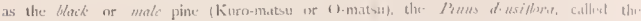

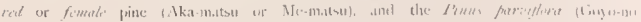

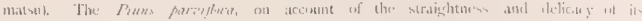

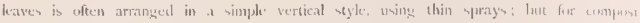

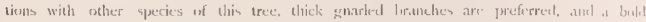
rugged charater stiven to the design. The pine in used mostly for arrimzemente in standing vessels. fir which it is saticl to be spreecially suited, but hanging complasition are vecasionally employect. A favouite treatment is that of a broad stump cut of frorizontally, with a thick twisted brancls springing from its basc. At all times the

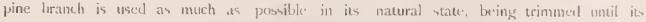

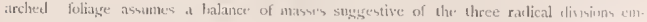
ployed in fower compositions, and the building together of separate branches, tonumin with other trees, is comparatively litte resorterl to. An example illustrating the pins

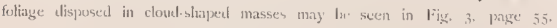

The eccasions for which the use of the pise is specially mprojuriate are at the New Year, at weaklings, at old-age celebrations, and sometimes, thonght rarely. 


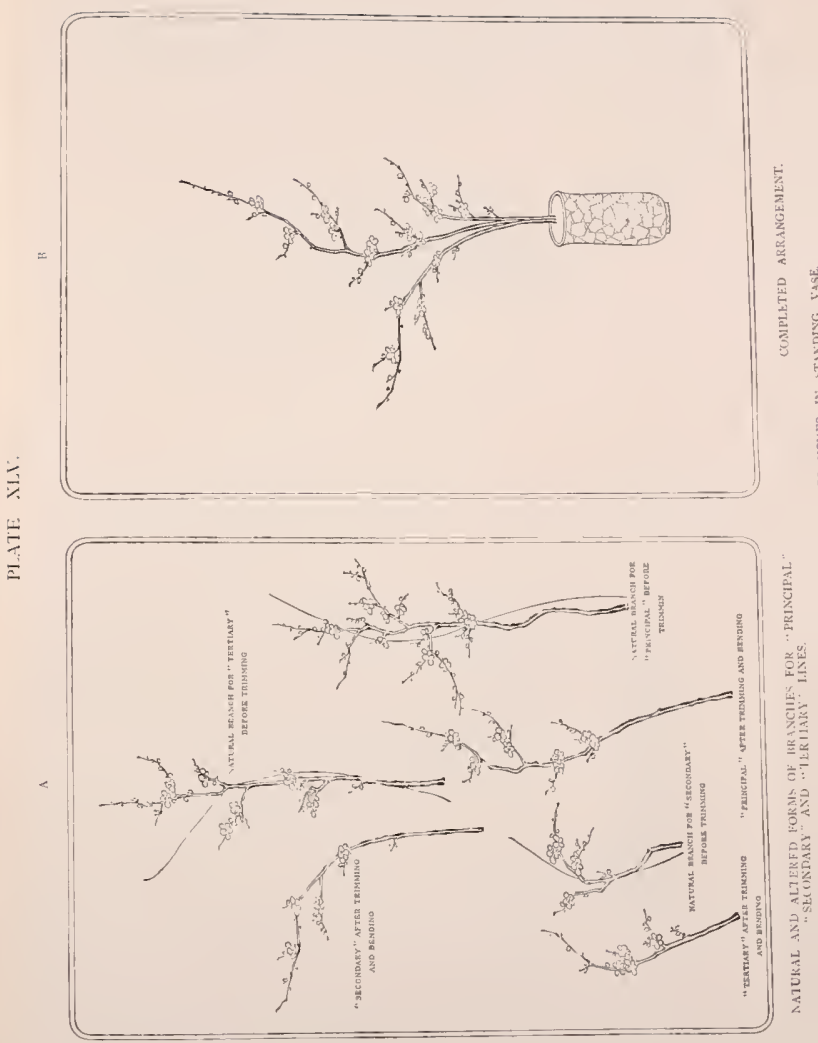





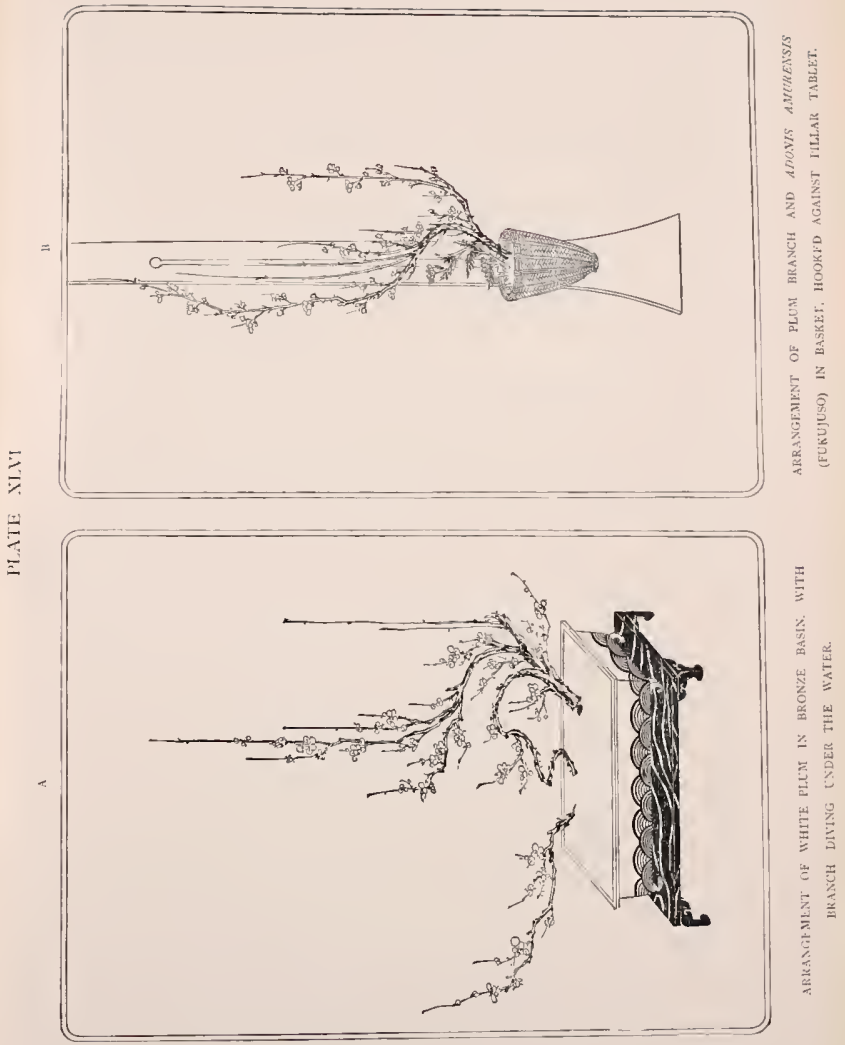





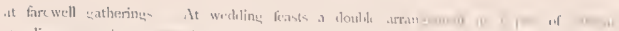

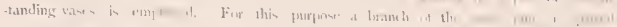

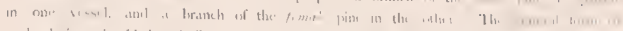

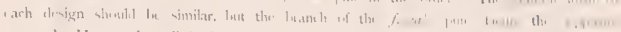

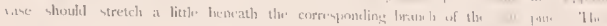

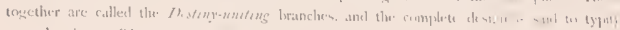

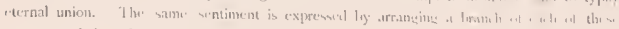

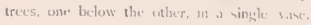

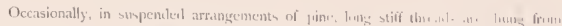

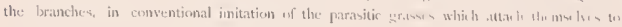

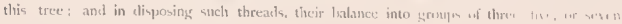
integular lengths is carefully ateniled to.

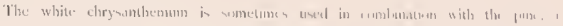

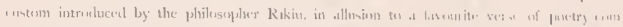

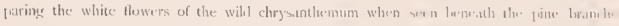

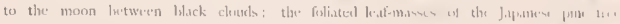

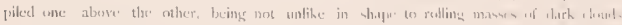

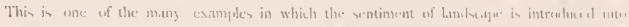

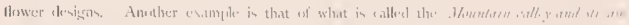

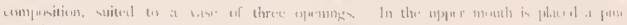

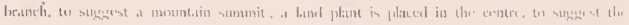

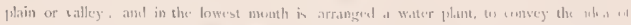

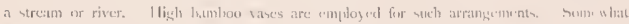

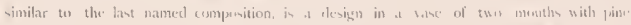

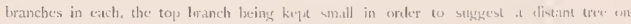
the lill-tup, and the lower branch full and elalonate twe represent a tren in the foregromul.

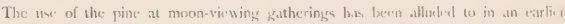

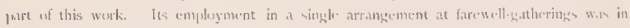

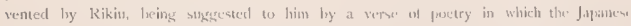

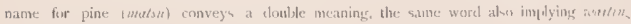
fir a lover's recurn, Many of the tralitions of the lilowes . Int alre based nipun and poctical allusions. 


\section{WKFWGEWLY OF FLONERS.}

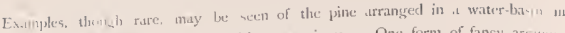
imtinsation with a water-plant snch as the iris or nurcissus. One form of fancy arrater. inent, called the Fuyl-pue. afece the famous volcanic moumtain I-uji-san. remains to he mentioned. In such a conijosition a bronch is leent es resemble the outline of Mlount Fuji. and is cembined with the other branches suxt folliage in such at manter as to give

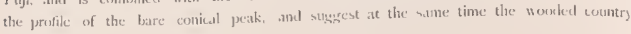
it its lase.

\section{ARRANGENENT OF BAMBOOS.}

In the Howwer. Art, the bambon is, strictly speakings regarded weither as a 4 . nor a Nan/. bat it may occupy the presition of either. It should never be placeel in a vessel made of banboo. In combination with other trees or plants, thin loranches or sprays of bamboo are often employed, but for simple compositions, a portion of the round stem or tube is selected, with a few lenfelad twigs attached. The top of such tules are cut off etither in a splayed or hotizontal mamer accorling to the occasion. If used at wedding feasts, the cutcing mest be hidelen by leaves, the sight of it being considered unlucky and sugrestive of severed friendship. These cylinders of frean hambar require very special and careful treatment in order to preserve their verclure and vitality for any length of time. They must be cut in the cool of the morning or eveningt. In the early monthe of stmmer, when the leaves are young, the stem in very succuknt, and no special treatment is required, but to preserve the freshness at other seasons, small holes are drilled beeween the knots of the tube, into which water is blown with the mouth. Sometimes sake, the rice-wine of the country, is added to the water as a stimulane.

Mostly three, but sometimes five twigs of leaves are left on these cylindrical stems, arranget irregularly. The leaves of such branches atre, in their matural state, crowded and confused, and they therefore requirc thinnings out, the witlered ones being removed, and the remaineler disposed in doublo or triphe stoups. Three different combinations of leswes are approved, that of the fish-taril, in which two of the lancetshaped leaves spreat out like the twil of a common lish; that of the Gedt-fist-tail, in which a central feaf is added, giving the group the resemblance to the triple-finned tail of a fold fish: and that called the F/ying weese shape, cotsisting of three sloping leaves, suggestive of the outline of a wikl goose in flight as seen from a listance. 


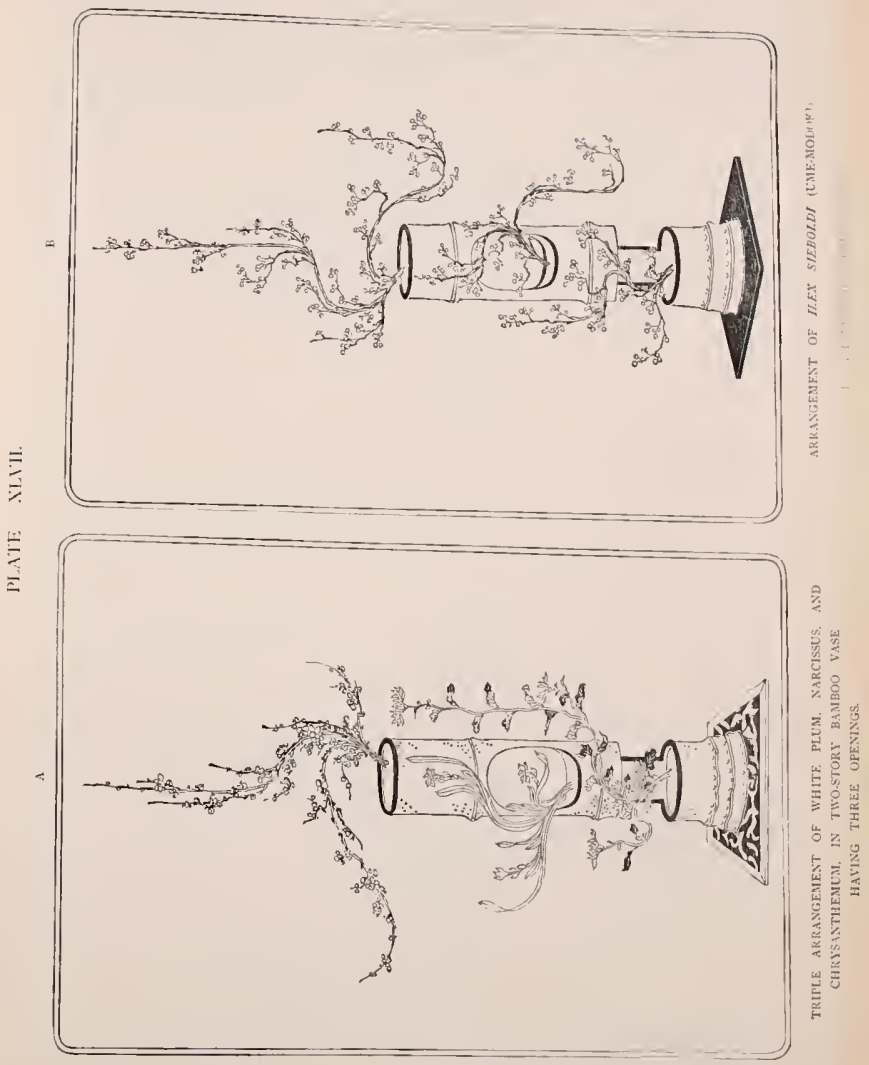





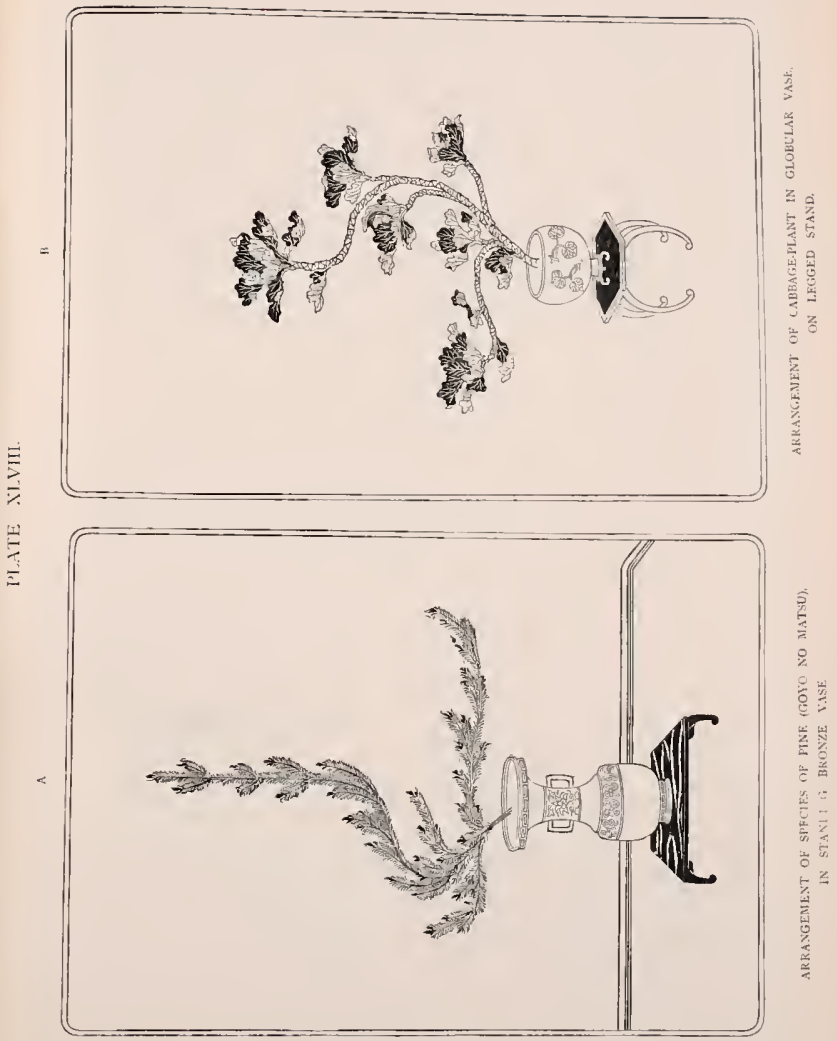




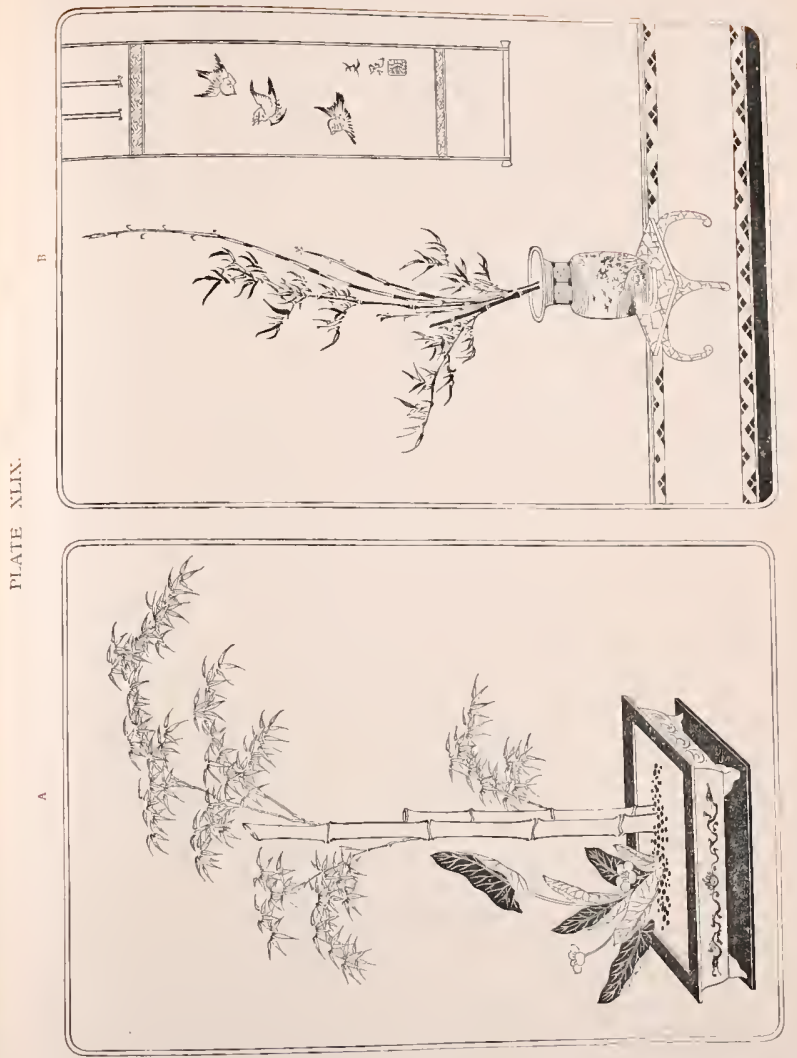



On some occasions, two tubes of green bambon, one whorter than the other. are combined, heing then called respectively the moke and fimate. The top of the femals or lower tulse should ine cut of horizontally, whike that of the urper tule should have a splayed cutting. The longer tulue has three knoty ur divisions, and two leaf-clad twigs which take the place of Irinteptal and Secondryy in the conposition. and the shorter one only two knots and one spray of leaves, corresponding to the Tirtinge. To dewigne placed in broad vesseds a bambon sprout (Take-1no-kos) if in seasm, may be added as an auxiliary. and it should $\mathrm{Ix}_{\mathrm{x}}$ fixed about two inches slistant from the main stems, generally in a slanting position and clese to an ornamental stone.

Numerous vases made of bamluo cylinder's have been described in an earlicr

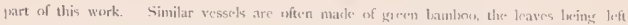
on and forming part of the floral composition arrangred in such vasts. The same carr is required to keep such receptacles fresh and sereen as is applied to nrelinary bamber tulues, and whik the upper and lower portions are cut out to form mouth for the purpose of receiving other plants, the intermediate yace between two knots is hored and sprinkled internally with Water and solie, In Plate XLIX. at 1, may be reen an arrangement

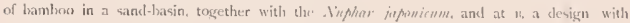
thin sterns of banzloon aloue.

\section{CONBINATION OF PINI, BAMIBOO, ANI PLLNT.}

Separats notice is given to the combined arrangement of pine, bamben, and plum branches, on account of thre importane attueherl to this triple alliance in the art

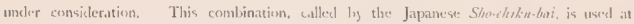

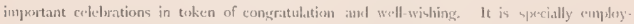

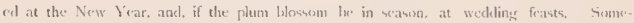
times the ermprastion is in a single verset. and sometimes three vases are used ench containing one of the three growths. If all three lie placed in one vase, the pine takes

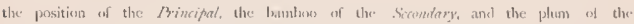
Firtiary: and if arranged separately, the pine uecupies the central jusition, the bamiluo is placed on the left, and the plum on the right. The plum branch shonld have its base

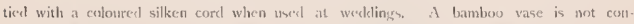
sitlered suitalile for sucls triple arrangenents, but a vesucl of green lamboo may be $\mathrm{cm}$. ployed with the pine and plum only, thus including the vase its-if in the triple combination.

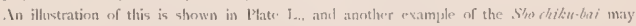
be soren in the same. Plate, at 11. 
12. HRKIYGEIENT OF FLOWFRS

\section{ARRANGEMENT OF WIILOW BR.INEIIFS.}

The nillow is employed for fioral deaigns on important occasiuns. It in the fitesenth dly of the cischth month to the first day of the thied month. Owine to the

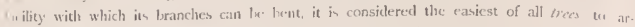
range after the Jayanese matiner, and ge actrally forms the sulject of first lessons in the Ar: Among the many varieties of the willow found in Japan, timse most used for floral oum

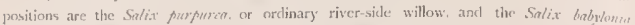
or weyging willow. When branches of the secping willow are rmployed. carn mast be taken to avnid an arrangement in which lines droop on loth sides of the same foum

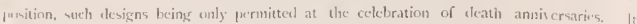
a common prattice to tie the leng trailing stems of the willow into a loose loop ur ma

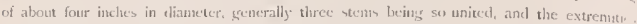
hargings down tw different lengths. The originator of the Enshin School is said to hin intenteal this style of composition. as he foumd it othernise difficult when arranging willuts branches in standings vessels, to prevent the lomy shoots ferm trailing on the fi... Anoluer sersion attribute the pratice of looping the willow to an old Clinese custum prevailing at farewefl me-ctings, on which occasions it signified fyimg-uf until the return of

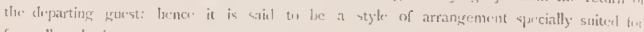
fur.well satherings.

The custom lucame afterwark applied even to hanging comprositions, which .11 stresally preferred for this tree, the idea bring that, because the willow grows near whiter. its branches should hang over laterally its if dropping over a steam. Firn in

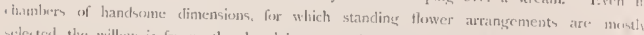

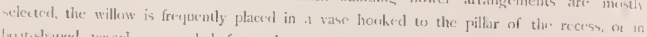

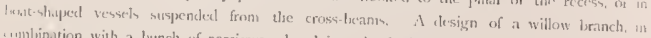

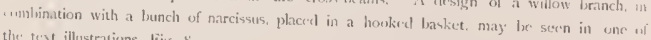
the text illustrations. Fing. $\mathrm{x}$.

The camerlias is the farourite flower for introducing with the willow: .

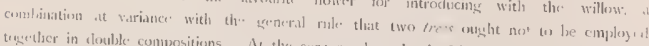

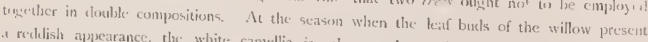
1 reddists appearance. the white camellia is chesen. but when these buds assume a

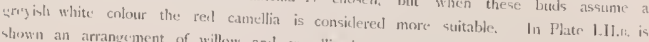
shown an arrangement of willow and camellit in at susponded bronge vesact of crescrnt 

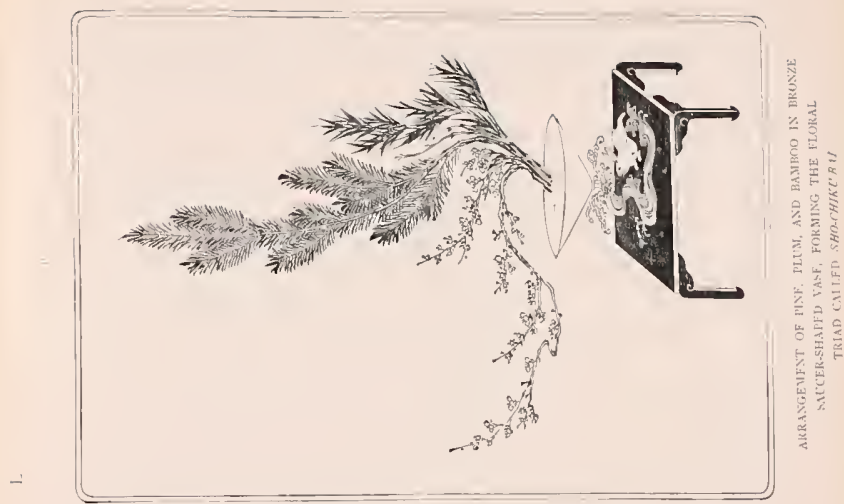

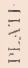





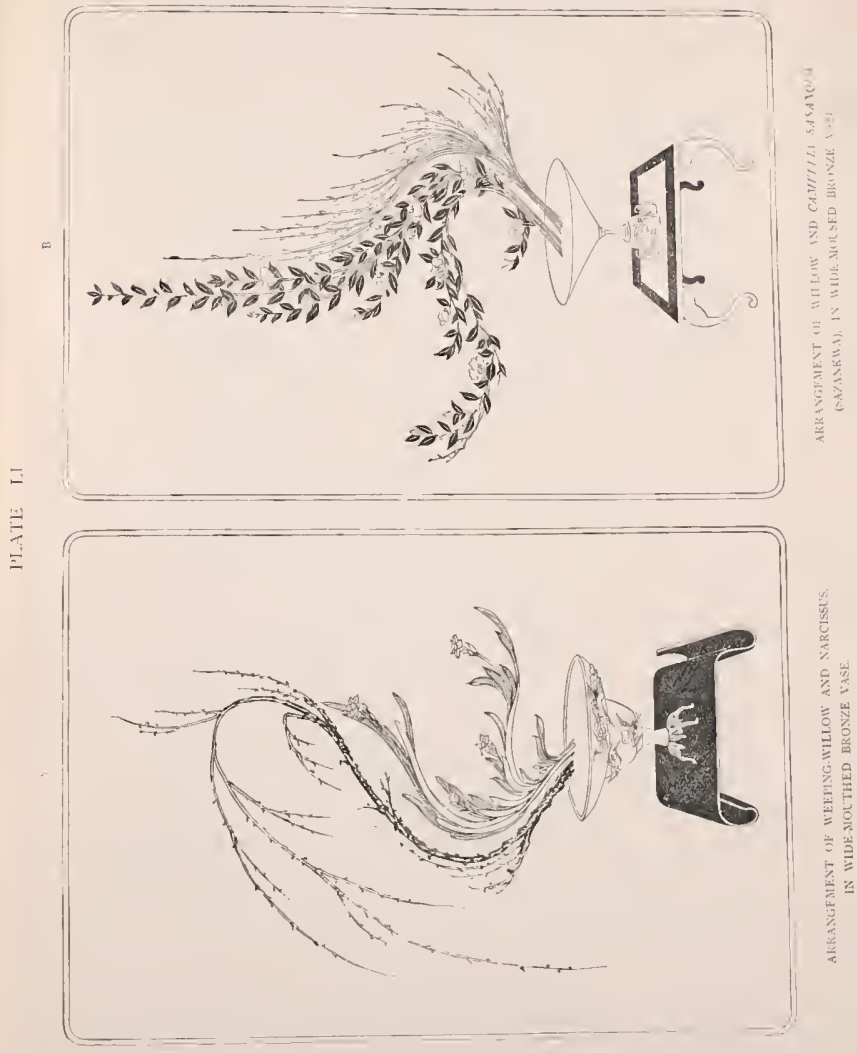



shape, and in Plate L., are illustrated two compositions, one onsisting of the willow combined with the Cimellin sasampur, and another of the willow and narcissus. If both are arranged in a two-staged bambon vase, the willow should be aloner, and the caniellia below. The narcissus and winter clirysanthemun are also occisionally used in combination with the willow.

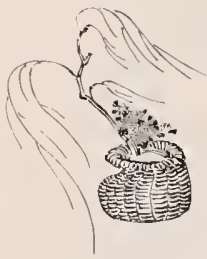

Fis 26

Three fancy stykes of emmposition are recognized For the branches of this tree, nantely, the willeat in fair weather, the sillow in swind, and the willone in smors. For the fait-weather style the Syring willow is used, and the brancbes are spreal as if just kept apurt by the breere; when the willow in wind is expressed, the branches are given a curved swerp, as if blown lack by a strong wind, (sce Fig. 26); and when the willow in snow is suggested, the stems are made to hang straight and heswily as if weighted with snow.

\section{ARRANGEMENT OF IVISTARII FLOWERS.}

The wistaria, as the first flower of Stmmet and the most important of blossoming creepers, holds high mank, thongh its colonr ronders it unsuitable for certain oceasions,-purple being associated with mourning. The species with white blossm is seldom used in floral designs. From its nature this plant is specially adapted to suspended arrangements, though it is also used in standing vessels; in the latter case, smme sort of frame is generally necessary for its support. The blossom-clusters are preferred before the $y$ are in fill bloom, lsaving a majority of buds and half-open flowers, with only three or four fully open.

Tubs and sand basins, such as water plants are placed in. are the only kinds of standing ressth used for the wintaria, which, it must be setnembried, grows in parks and gardens on trellises overhanging a like or stream. The ilka of such water-secmery is conveyed by means of these broad, fiat vessels, and stones are often introduced to add to the impression of landscape in miniature.

An arrangenent with ornamental stones is shown in the accompanying woodcut, (Fig. 27). Such stones are grenerally three in number, with three distinct characters, 
distinguished by the names theaven, Earth, and Mankinel. The principal stone is high and pointed, like a moustain in shape; the thircl stone is flat and horizontal in claracter; and the second stone is of intermediary form. Other stones of minor importance are often added, but not so as to sletract from the three essential oncs, and the complete number should always be an oskl one. Tlye steru of the wistaria shoukl spring from behind the principal stone. Water plants like the iris and Aiphorr jinpmicunt. and land plants wich as the Caleudule officinalis, Bijinso, and Aspidistrn lwrith, are sometimes combined with the wistaria in shallow vessels. If land plants are introduced, no stones should be employed, these being only resorted to when water scenery is expressed,

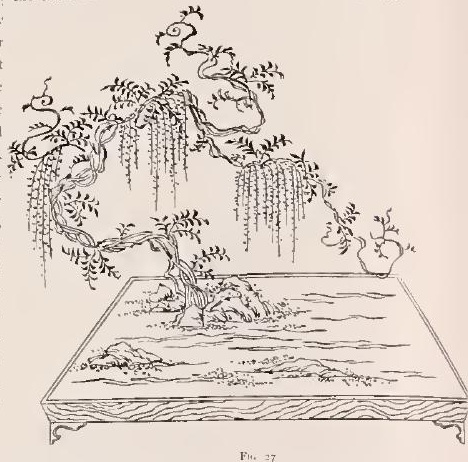

I composition with wistaria, irises, and Niphar jufumenen would be arranged as follows: The wistaria should $l_{x}$ phesed to the left of the vessel, at the sicle of a high stone, $-\mathrm{as}$ if it were on the slope of a hill with its branches stretching over and its Howers reflected in an adjoining lake or stream. It should have five, seven, or nine clusters of blussoms. Then, at a distance of about six inches, a gromp of the leaves of the Wriflin. juponicm is placed; and about four and a half inches from this plant are arranged the irises in a simple composition of three or five leaves, one of them curling over and dipping into the water. The ramaining stones should then be clistributed in different parts of the vessel, but so as not to interfore with the water plants, which must be placed in the free waterspace.

The nature of the wistaria-creeper prevents its stems being arranged in distinct lines in accordance with the general methods of Horal design, but the rules for Frincipal, Siroudary. and Tertinry. are applied to the grouping of its blossom-clusters. In hanging arrangements the Sircamer assumes great importance. 



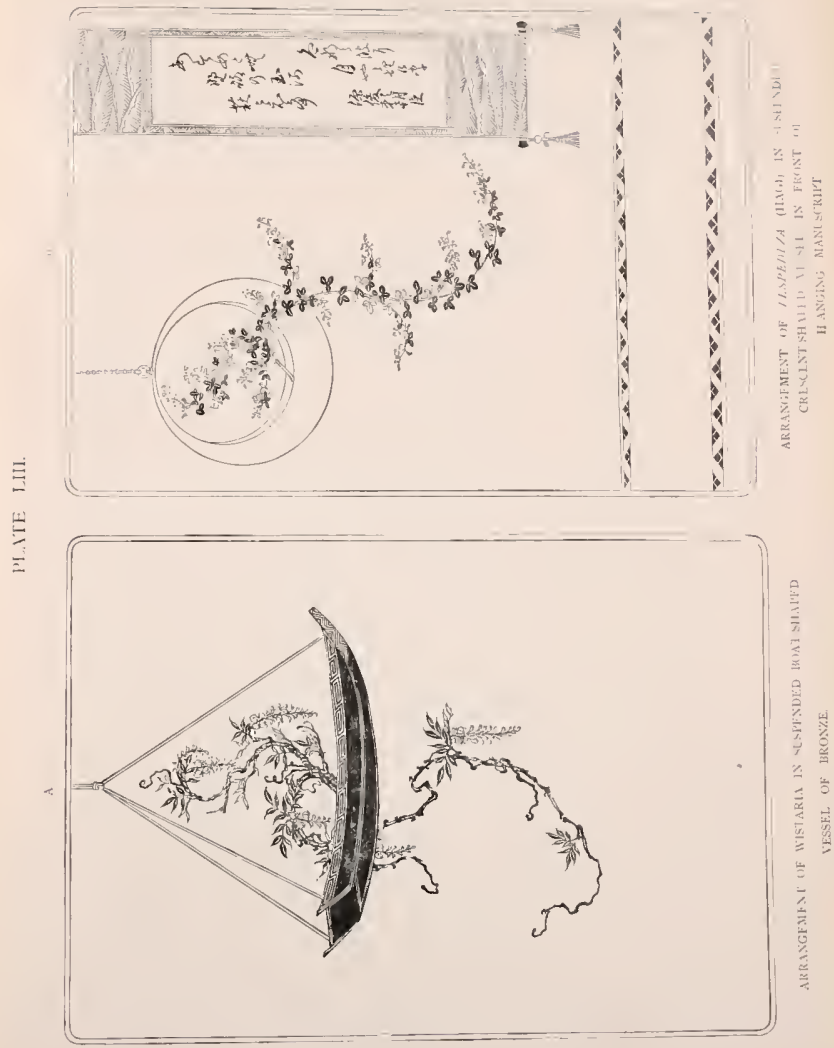



Examples of suspencled compositions my Le seen in Plate L.1L1. w, where the Hower 1. placed in a bronec boat-shalge vesset, and in Fig. 16, page 76 , where it is arranged in an inverted bell twining round a branch of pine.

\section{ARRANGENENT OF IRISES.}

Several kincls of irises are emplayed for floral arranguments, the most important

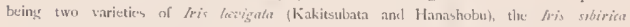

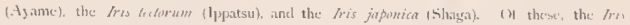
lecigatu Fish. atr. Aempfers, which the Japanese call Kakitsubats, is the favourite. This plant, though belongring particularly to the early summer, fasts thromgli several seasons, displaying a special clasacter of growth at these different perisds, In Sinsing. the leaves are stiff and strajght, and the flower stens are short; in Sumner, the leaves are more full and spreading and there is much spirit in the Howers; and in Autumn the Hower stens are long and the leaves bent and curled. These peculiar characteristics of the plant at cliferent seasons must be shown in the floral arrangements. The leaves of the iris, like those of other water plants, are considered the most important frart of the composition, anel they must be well selected, all witherecl ones being cliscarchet. They are pluckecl as they stow in their sheaths, separateel, and then artifially connected into groups of twe or three, lyeings attached with saliva. These sets of leaves are used to form the different lines of the design, names being given to each accorcling to its position and function in the composition. The main leaves form the Principal. Sicautiory, and Ferliary lines of the arrangement, to these being added two or three flowers and other auxiliary leave's. I'lutes I.V. and I.IV. demonstrate the: methots of taking to pieces the clunps of leaves, re-arranging them, and combining them. with the addition of Howers and buds, to form a simple composition.

The usual numerical proportions betwcen leaves and flowers in an arrangement of irists, are:-one flower with three leaves, two flowers with seven leaves. two flowers with fiftecn leaves; three flowers, with thirten leaves; and five flowers with eleven leaves,

Taking, by way of example, a comprsition of two flowers and seven leaves, the following is the methot of arranging. First, three leaves are placed in the position of the

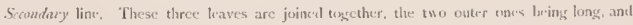
the central one short, as if just sproutingt out from between the other two. Above this group of leaves a fult-blown flower is pliced, with one long leaf addect, which oxcupies the. position of the Principal. This is often called the Cap-leaf. as it crown the whole. 
Behind it a smaller leaf is added as an auxiliary. and at the side of thin a flower bud is introrluced. Nevt, a small leaf is placed in the position of the Tertiery. technically called the Ifater-dippeng-lenf, because its tip curls over to the water of the vise: and combincd with this is adkdud another small leaf called the Dert-supporting lecof. because its biale shouk be slightly concave above, with an upsward tendency.

In making a composition of eleven leaves and five flowers, three leaves are put in for the Sccosdary, as before, and a flower is adted. Behind this, and rather higher up. the Cap-lenf it placed, and with it is arranged the bighest flower as Principal, with two other auxiliary leaves. Then another flower is adled between the Irmeifal and Sccomdary blossom with an additional leaf below it: and on the opposite side of the composition, it little lower than the fower of the Secondary, is placed another flower with two leaves adjoining. Still below this is added a bud, for the Fortiary, combined with two leaves,the Walcr-dipping-leaf and the Drel-supporting-lenf. Of the flowers mentioned, two should be in full bloom, two partly open, and one in bad.

Reference has lacen made to llower arrangements in broad flat vesseds in which trees or plants are disposed side by side in clivided clumps. The distance between such

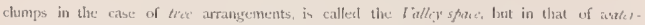
plants it is calied the fish-lrovelfing-dislante. because a lake or stream containing sucly plants is suggested, and the space left is just sufficient for the passage of harge: fish. Such interval should measure about one tenth of the height of the largest group of plants.

In a divided arrangement of irises in twe clumps, one slonkl be large and high, in the Iirtical-friangle style, (see ligh. T, page 47). and the other smitl and leaning. in the Worisontat-friangle style, (14ug. 2, page 48). The main group. placed to the right side of the basin, may have sever large leaves, a llower, and a bud; and the other group, six small curled leaves, and one bud.

The Japanese florid artivt delights tor suggest in his compositions some notect natural l.melserpe. The mout famous spot in Japan for cliopliye of the iris, is Vatsuhashi. in the province of Mlikswa, where there is a river with cight tributary treans crosscel by as many briclyes. An artifical flower arrangenent is sometimes made in sugrgestion of this natural view, by using a very large, shallow basin in which are placed white pebbles to represent the river and its eight branches, divided by black stones, flled in for the lancl. 


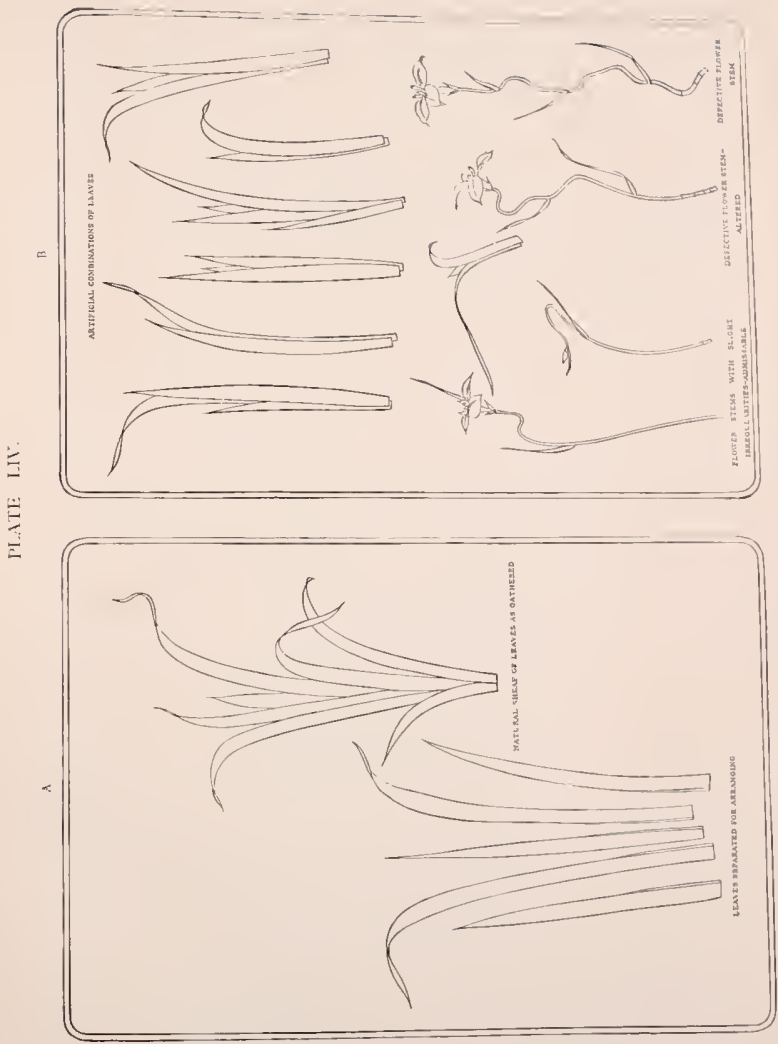




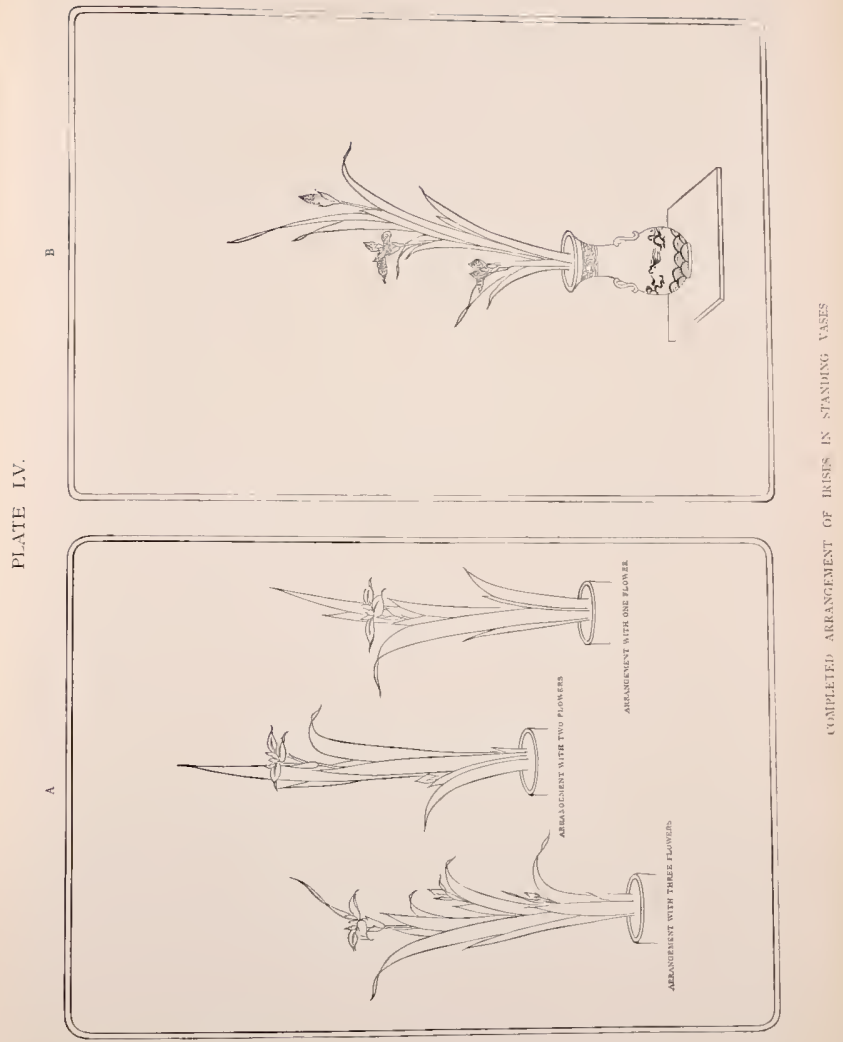



The irises are then arranged amongst the black stones in divided groups. Each grenup is composed separately with a different quantity of leaves and a proportionate. number of buds and flowers. Some bunches are clisposed vertically, others in a move kaning style. ancl numerous pairs of joung leaves are arranget between, th ternomect the whole. This amangenent is one example of a kind of flower composition almost resembling minjature gardening. The plants are held in their position by the sand and pebbles, and no other visible fistencrs should be employed.

The firis sibirica is arranged in a somewhat different manner, the flowers being placed ligher than the leaves. whereas, with the fris latigata, the blacles rise above the blossons. Another peculiarity is that the leaves are arranged in regular pratiations and not in indented triplets.

The lris may be seen in Plate LVIl, at $x$, arranged in a large lisket together with a branch of fir, and at 1, it is shewn as an indepindent composition in a small bamboo vase. One of the subjects in Plate XX1\% is that of flage placed in a Well-frame vessel, and helel by a metal Crab-fastens?. In Plate LVII, a larger design of irises is illustrated, forming onte side of a paired floral arrangement, the flater furtarions heing placed in another wase opposite: 10 it. The iris is often arranged widh other water-plants, such as the Nifthar japonicmm and Rhater japonica, in a broat vesset. It is also to be seen combineal with trees, such as the wistaria, maple, ancl Nindina doncsticis.

\section{ARRANGEMENT OF PEONIES.}

Two elistinct sarieties of this plant are employed for flotal derights, nimely: the Promia mantan and the Parana albifora. The former produces blossoms of immense siac, and is generally artanged in wide-moutherl bronze vase's or antique lluwer-haskets, nor onluer

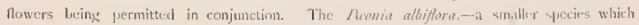
gones by the name of Shakingurkn, - maj be used in combination with branches of flower-

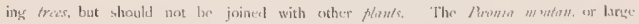
peony, is une of the three tlowers to which are attriluted rognl rank, the cother two buing the cherry and the lotus. It must therefore be used alone: and places in the position of honour in a chamber,- - that is. on the dais of the principal recess. It should never be arranged on a shelf or in any secondary jlace, and all other flowers must be excluded from the room which it is used to decorati. This rull is profussedly followed out of 
respect for the peim, 20 queen of all flowering plants, but its luxurions characte really renders such practice consistent with purcly artistic considerations, the adcition of nther material being calculated to produce a surfeit of richness and elahoration. The employ ment of the cherry blosson is also subjected to the same restrictions,

It is customary in ananging the large peony to introduce between the principal stems one or two black withered twigs, which. by contrast, enkance the appearance of the leaves and flowers. As the blossoms are mastive and heary in character. dacy should be sparingly introduced between the leaven, marcly more than one full-blown blossom being used: the remainder consists of buds and partially nopened llowers. For the ordinary composition in three lines, five of seven blossoms nay lxe employed, one tor the Iranespid. one for the Scomtary, one for the Firtany, and the rumainter distributed in intermediary positions. The leaves are carefully disponed bn as to frive support to the Howers. and in some cases are made to cross in front of them. The leaves surrotinding the Secondary blossom should be larse and closely grouped. They are technically called the Lerm-thing leaves, because the presence of the lion, which is associated with peonies in decorative art motives,-is supposed to be suggested behind the thick foliage. A flower bucl should be used for the Teviary, with the acldition of strong young leaves.

The Rironia albiftora, or small species of peony, is not letel in so much honour as the larger kind, and is often combined with other plants. In the case of this plant. half openet blossoms are prefered for the Jrincipal, fuil blossums for the Srontary, and buds for the Firtiary. The peony is sometinses arranged in wide basins divided into sejarate groups.

Illustrations of this flower, arranged in different kinds of vases, may be seen in I'hate LVIII, at $A$. and at r.

\section{ARRANGENENT U: LOTUS HLOIIERS.}

As has been stated in an early part of this work, the lotus plant is associated with the spiriti of the deach, and is therefore considered ont of place as a decoration at festise gatherings. It, however, holds high rank in the Floral Art, being resardecl as king of the flowers of India the source of Buddhisns. When employed on the dais it must be given precedence of all other flowers. and it would be consilered a gross violation of taste to combine with it the peony, which the Japanese regard as the royal flower of China. In the case of the lotus plant, the leasee 


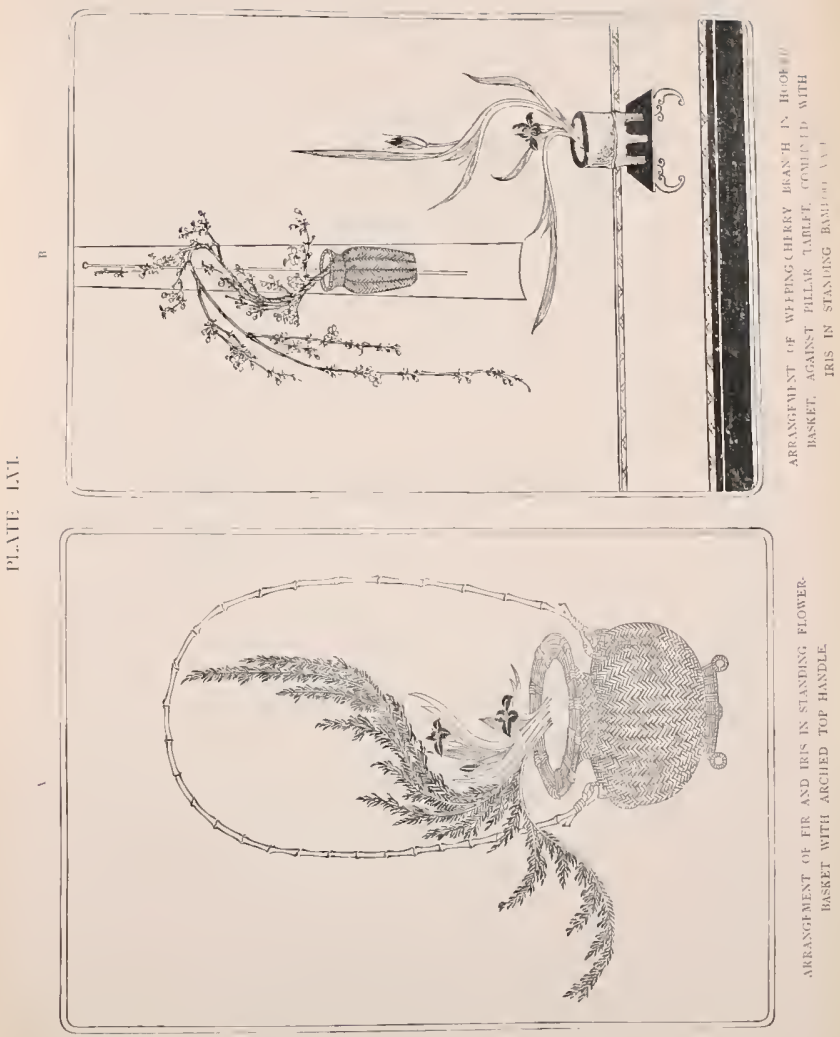





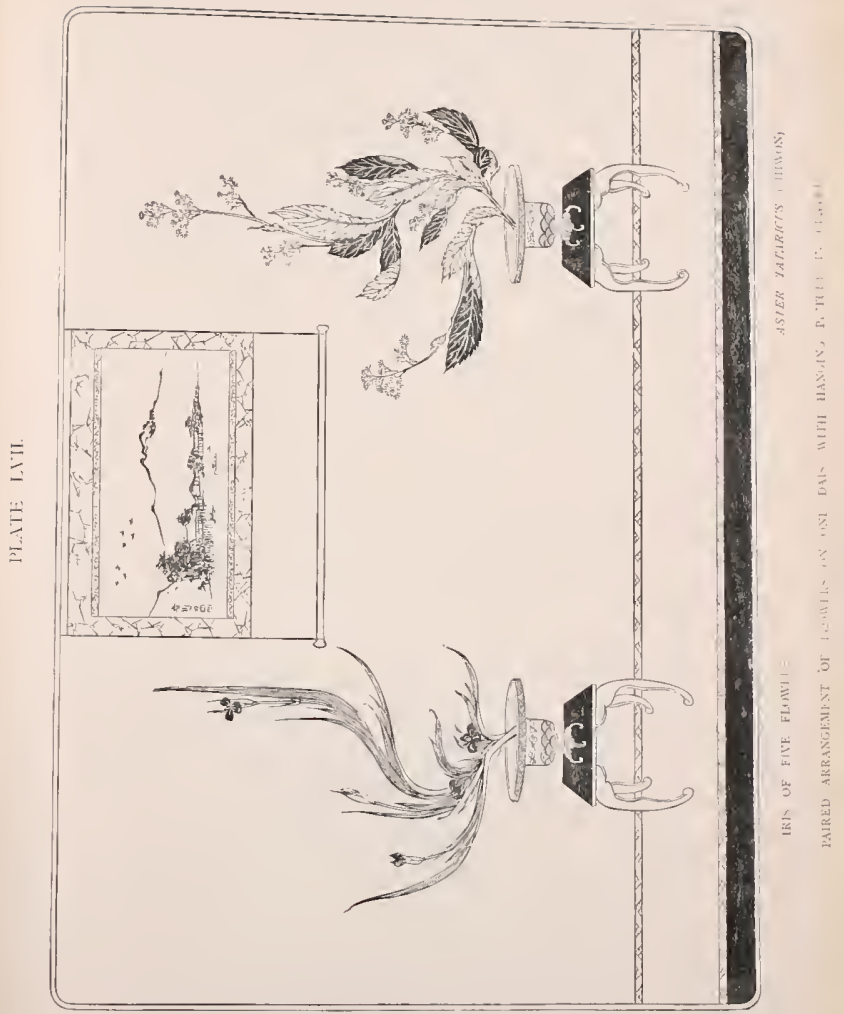





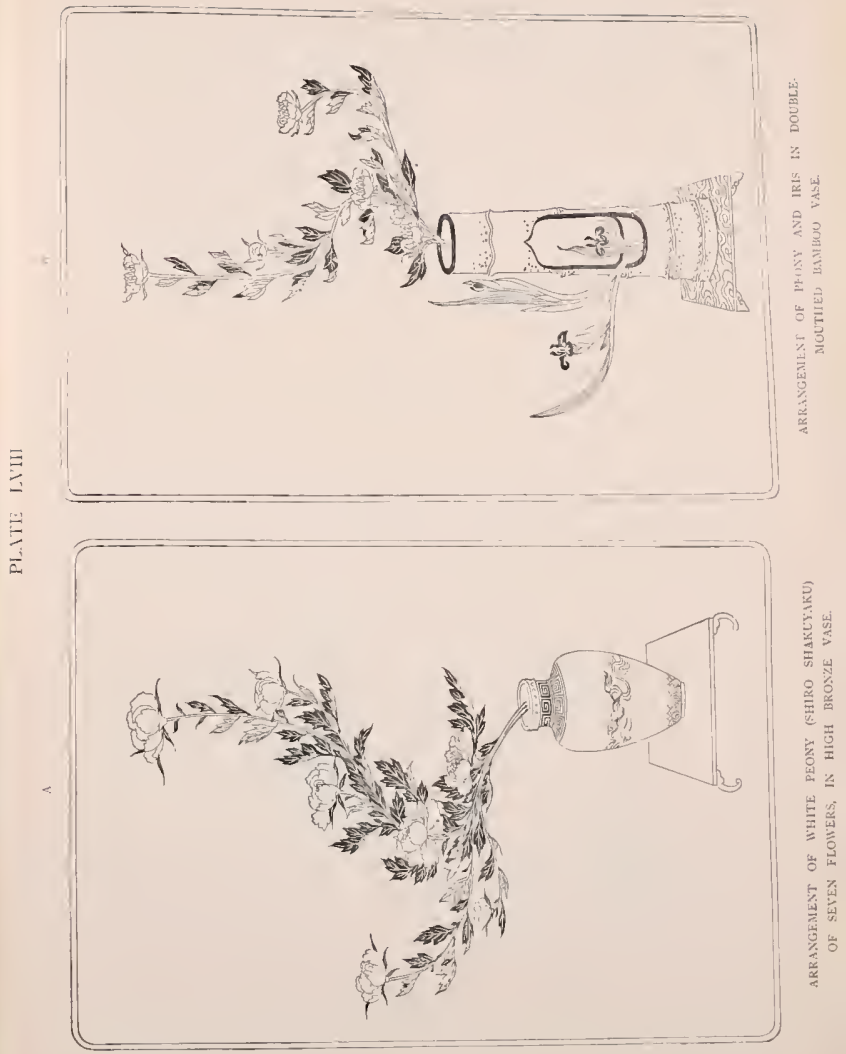





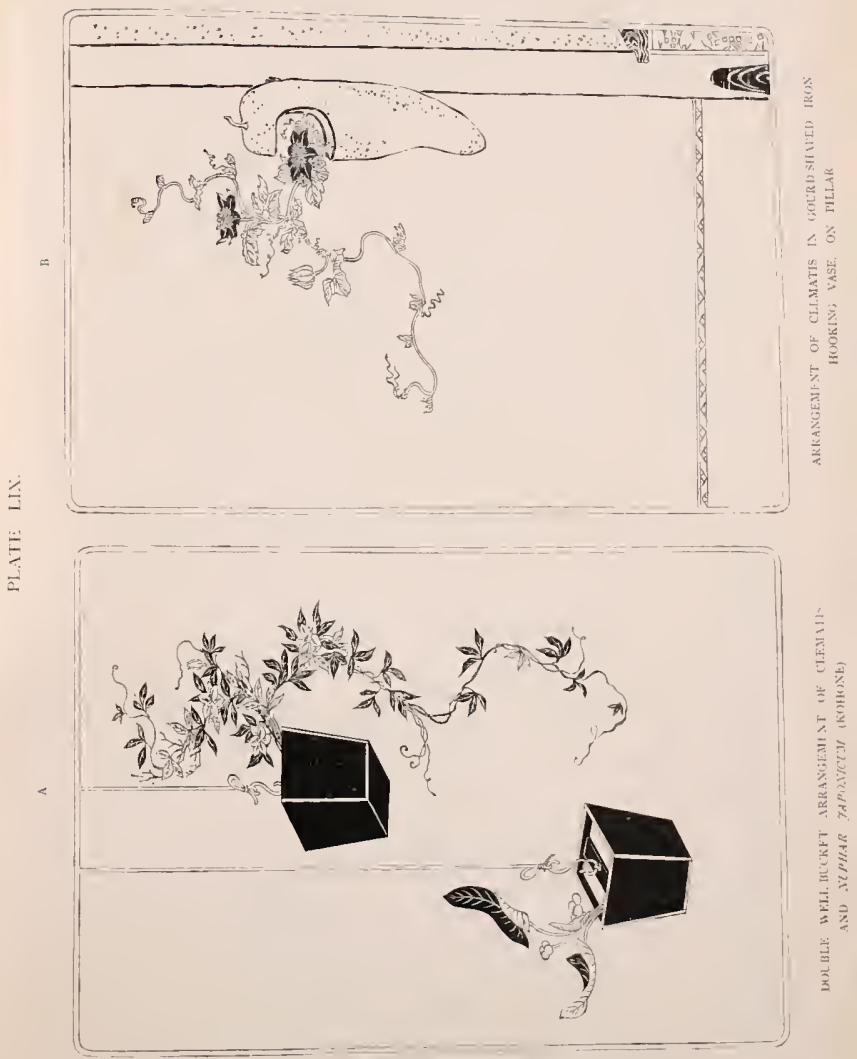




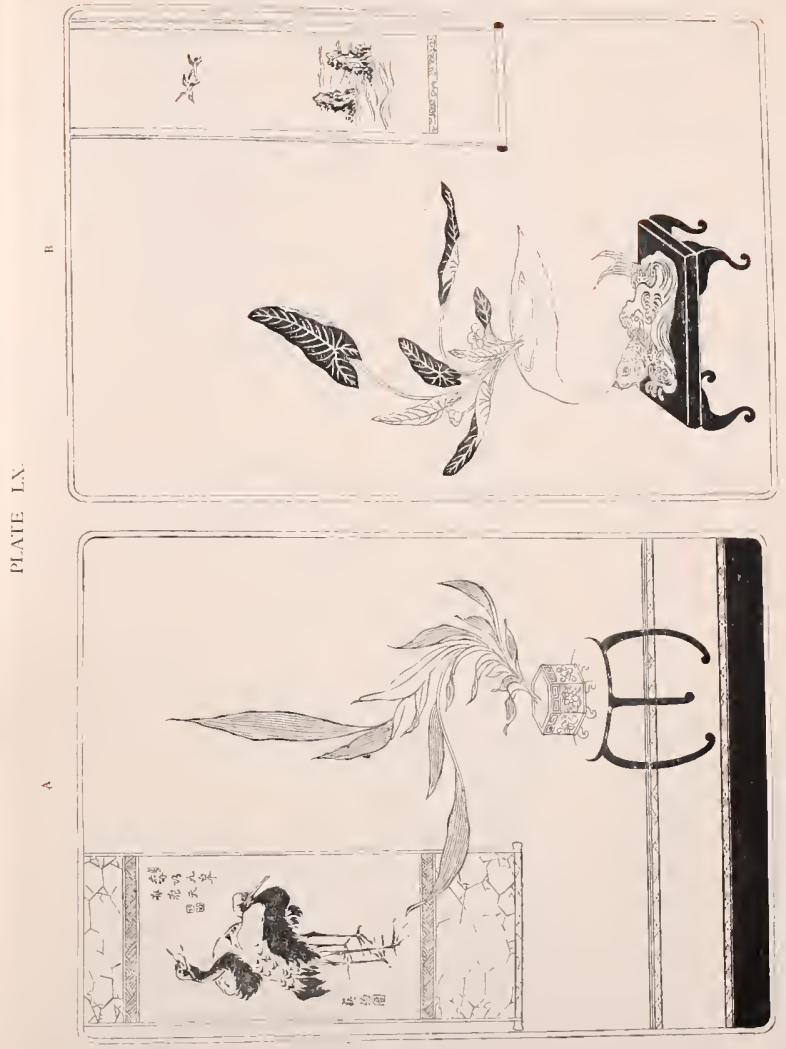

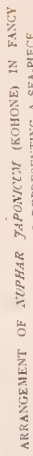

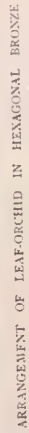





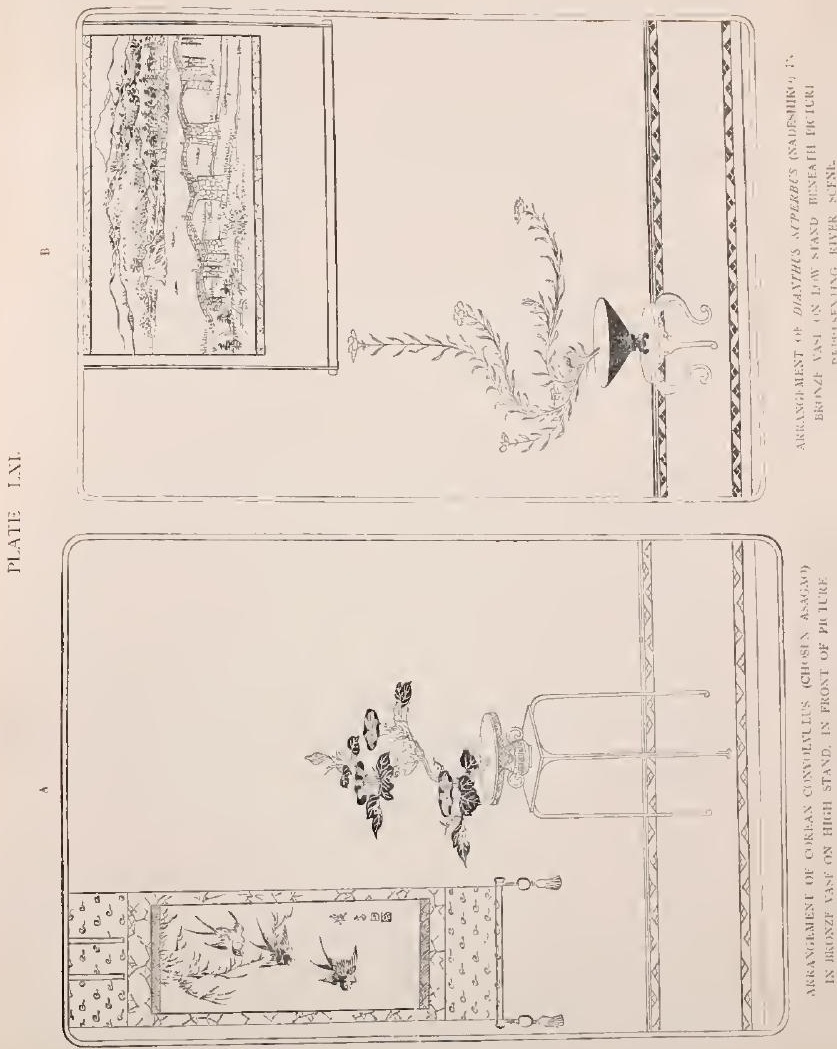



play the most important part in the composition. this being a rule which applien to nearly all water-plants. The lotus leaves should be selected to eyperss the ickst of the three Buddhist divisions of time-present, past, and future Jiav time is represent ed by a partly decayed or wom-enten leaf: Present fimic, ly a handsome ojen leaf,often called the . Firror-leaf, on accoust of its resemblance in shape to that of a layanest mirror: and future time. by a curjed leaf not futly open.

This plant should be arranged in a wide mouther vase. Sitnd-brim, or Thl, in which ressels it is often united with other water-plants. As an example of usch com. pound arrangements may lye described a combination with the iris. Ainflar japponicum

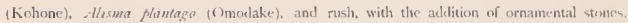
so as to form a sort of miniature lake scene. A greneral rule applyings to floral desizis in browd basins, is that tall plants suggest shallow water. whilst those short in growth saggest decp water; and as the lotus flourishes in comparatively derep water. the stem of its leaves, in a compound design, are kept sloort. The idea of lizimital Scondary and Tertiary is followed in the disposition and character of the leaves: for the Krinctat. in large round leaf being employed, for the Sccondary a smaller leaf, and for the Terliary a young and curled leaf. All open blossom is introlluced botween the brincifal and the Seramdary, and a thower bud, kept low in position, is placed between the Primifal and the Tertiary. The irises are nest arranged at a distance of about swo and a half inclece from the lotuses, an open flower being used for the Primigal, a half open flower with three leaves for the Scrondary, and below, in the place of the Terliars, two or three morr small leaves. The Nuphar japonicm is next placed in position, its Butripal consisting of a large oval leaf of interesting shape, and below it a half open flower occupying the place of the Sccondary. whilst for the Terliary, a yeung and only partly opened leaf is used. The meshes are then arranged in five or seven bunches, and the Alismat Nantage is afterwards introduced. separated from the rushes by a short space, with two leaws having a bunch of flowers between them, the composition being similar in style to that of the Nuphar japonienum, but bent in an opposite direction. Of the five plants in this combination the lotuses and rushes are disposed in the lertical-triancle style, and the others in the Itorisontal-trangle style. After they are all arranged in pusition, thr stones are added to connect the whole group togrether. 


\section{ARRANGEMENT OF CIIRYSANTHEMLIMS.}

I horal compositions with chrysanthemums are considered very difficult to arrangs
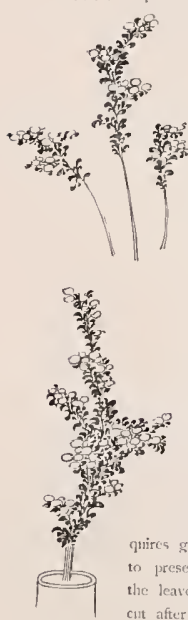

516. 38.

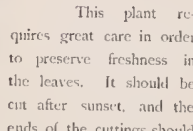
ends of the cuttings should

be burnt to charcoal. and placed for a considerable time in water before wedging, so as to prolong their vitality: The stems are brittle, and need great skill in bending to the desired curves, very refractory porshows an arrangement of Atrtumn chrysanthemums, which is altogrether more mature in style, the character of Autumn vecetation. skiffully: Of this flower there are many kinds, some of lares ancl sonse of small blossom, the latter presenting least dificult in treament. There are rarieties peculiar both to Sunmeand Autumn, and also a later species exists called the M"inte. chrysanthenum. Characteristics of grotvth at the differen seasons are faithfully initateal in Horal compositions. In Fig 29, are shown there separatc sprays of the small Summe clirysanthenum. and the same combined to make a florm design. It will be observed that the lines of this composi tions are strong and vigrornus, corresponding with the charac ter of Sumumer growth. In contrast to this, Fig. 31 in accordance with
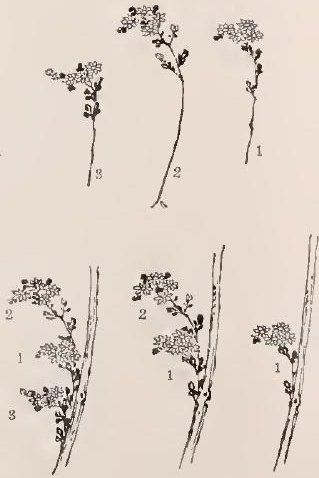

$\mathrm{Fin}_{2}, 2 \mathrm{~g}$. 
tions being softened by heat. The blossoms of the small clusysanthenum, as shown

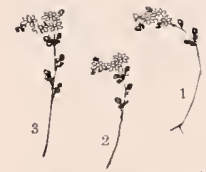
in Frigs. 28 and 31 , are massed in sroups, generally cunsisting of an uneven number of flowers, Figures 29 and 30 illustrate the methods of gromping togeches small chrysanthemums as auxiliary to a bouncls of some trec, in a mixel composition. In Fig. 29, three sprays are tsed, and in Fig. 30, five are employed. With the species of large flower. the blossoms are isolat.

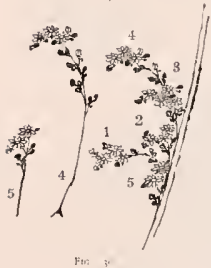
al and of limited numlyer, each sten bearing one, two, or at most three flowers. There are said to be seven faults which must be guarded against in the dispesition of such large flowers. A blossom trust not be turned away so as to present its

back in the composition: not must it turn its full disc to view: flowers must not have stems of the same length so as to he exactly in a line: nor must three blossoms be arranged in a triangular form ; the disposal of blossons in regular steps is objected to: colonr-santuriching,-as previously described,-is another fault to be gtarded ayainst: a large open blossom should not be put at the lower part of the composition: and Howers should not be hidden by leaves. To correct a tendency to the ahove faults small hamboo skewers carefully hidden are employed. The illustration Fig. 32 shows the method followed in arranging plants having large blossoms or large clusters of flowers, The flower shown
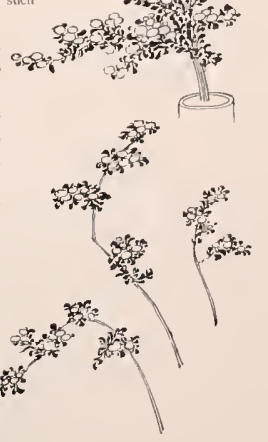
INE. 31 . 
is the patrinia. There are seven principal flower clusters, which are essential. marked A. B. C. D, E. F, G, correspondling to the masses of a seven-lined componition.-Primcipal. Sroudary. Support. Terliary. Sub-priucipurl, Side, and Frunk: and the acklitional thewer 1. e, f. s. and others, are merely auxiliary and arlded at discretion, to avoid hareness.

In adkition to the special form of arrangement suggested by the peculiaritum of srowth at different seasons, three general styli's of compexitim are recosmized for flisfal designs: namely; the finished style, the inlermedzary style, and the rengh style. Therse distinetions correspond with, and are described by: the same terms as those applied to the square. and grass or running Chinese ideographs, and refer to differcant dugrees of chaburation or setetimess. In Plate XLIII.A. are shown chrycanthenums arranged in a rush style in combination with other fowers. llate 1.XII, exhibits two composition of chrysanthemums in $\mathrm{x}$ more firished style, one representing these plants clisposed in five lines, with seventeen blossonis.

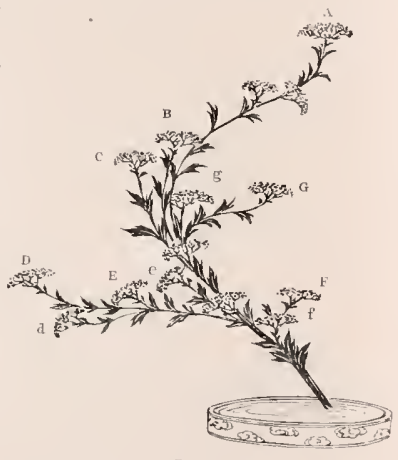

in a fancy bronze vase, and the other showing a triple design in a bamboo vase of three mouths, with fourteen blossons in all. A rough arrangement of these Howers in a special kind of basket is illustrated in liug. 9. page 61.

\section{IRRANGEMENT OF NIPHAIR FIPONICLM.}

The Auphar joponticustr, on account of its beautiful oval leaves, is a fiwourite

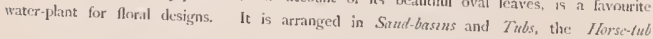




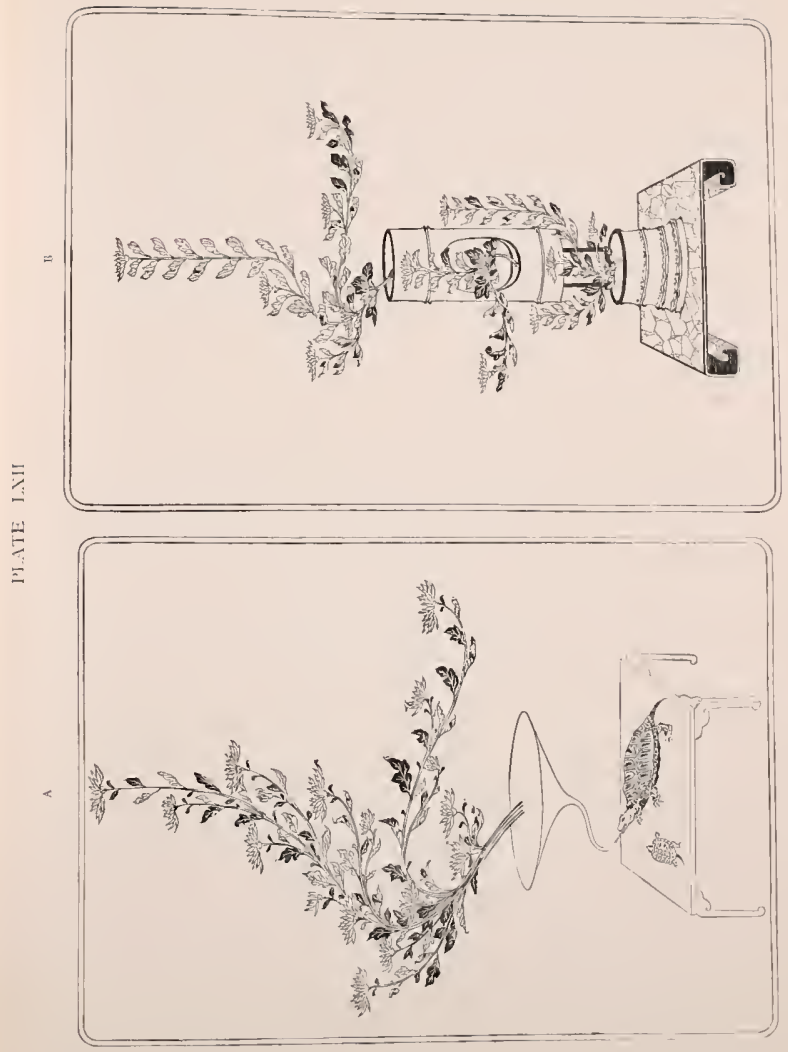


heing by freference sclected, and the floss's.bnt, or some other fancy fastener of metal employed. The largest leaves of this plant lrang horizontally on their stems. and are gracefully curled at dieir edges like those of the totus, The stens are generally arranged as long as possible to convey the impression of a plant which grows in slallow water. This rule becomes specially insportant when the muphar in combined in a single vessel with other water-plants which thourish in less slallow pools; such rleep-water plants being purponsety kejt as short as prosible and placed more centrally so as to suggest the illea of greater depth. Though the flowers of this plant are snall in comparison with the leaves, their position in a design is much studied, and the leaves are arrangel in reference to them. Stlon more than two or three are introduced into a single composition, and these, combisting of a but and one or two hali open blossons, ate grouped between the Praciat and Fintrery leaves.

For the Principal, a large and slightly curled tear is employed, placesl higher than the rest, ancl extending to the left of the design; fir the Sicundiny. a somewhin simaler leaf is fixed leaning to the sight; for the Ter/hrw, a small colled leaf, uedmically: called the Horti-luef, - is added below the Primcipal, and heneath this is a joung bent kat called the Haker-tanching-leaf, which should be only about an inch above the water snrface.

The slender stems of this plant when arranged as nbove show a deficiency in width, and it is 115 hal in broad water-vessels to introduce a secondary clump at the side, removed from the main composition by the space of a few inclies. Ilhis may consist of a single flower and one small lear, kept very short as if just sprouting from the water.

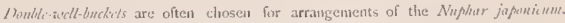
In Wlate LIX.A. may be seen such a composition in the lower of 1 puir of suspended Ifell-buskels, the elematis heing introdiced iato the uppers one, in at langing style, with

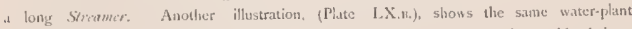
disposed with seven leaves arsd two flowers, and in Plate XL.X.A. it is combined in a large Sind-besiu with a high group of hambons. 


\section{ARRANGEMENT OF KERRIA $\mathcal{F} A P O I Y C A$.}

This modest plant, with its long sprays of yellow blossonss, grows in abundance near the banks of rivers, and is specially associated with the scenery of the Taniargawa, at Ide, near kijoto. The accompanying illustration, Fig. 33. represents a composition made with the Kerria in a broad Saud-lasiz, intended to suggrest such river-scenery. The stems are lyeld by long cylinders of bamboo basket. work made in initation of the $\gamma_{t-k a g}$, or huge baskets holding boulders which are used to break the current of rapid streams, Stones are added to complete the idea of the natural view.

is also frequently joforita in suspended receptacles and Boats, the stems having a

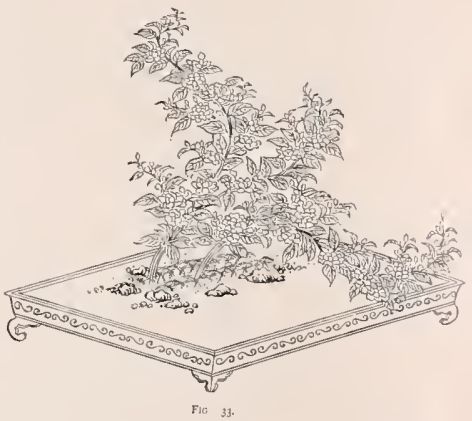
leaning character griven them with the object of preserving the notion of llowers hanging over and reflected into a stream. The popular fancy that this plant bears neither seed nor fruit prohibits its employment at wedding cerenonies.

Plate XIII, illustrates a defective and a corrected arrangement of the Kerria in standing vases, and Plates XXXVI, and L.XII. show the same flower disposed in a

\section{ARRANGEMENT OF NARCISSUS FLOWERS.}

The narcissus takes an important place in floral arrangements during the Winter and Spring montlis. Available at a time of year when other blossoming plants are 
scarce, sweet in scent, and of golden colour. it is considerably prized by the flower-artist. Varietics of red, white, pale blue, and double blossom. exist, but with the exception of the white, which is sometinses used on ceremonial occasions, these rare specimens are scldom scen in floral compositions. The blade-shaped leaves of this phant reccive chief attention. and are used in such greater number than the flowers: they are removed from the sheath, and are separately pressed and rubbed on a board to take out the excessive twist which the heat of the sun gives them in their natural state, a single curl alone being per-

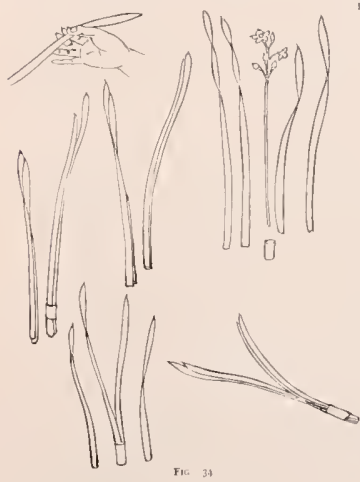
missible for leaves eimployed in composi. tions. The younger leaves are easily smoothed and straightened with the fingers, but the large ones are refrac. tory and require much labour and patience. Thus altered they are attuclied together in pairs consisting of one long and one short leaf, with the longer 1) Jade in front, or sometimes in triplets. and these busches are enyployed for the different lines of the arrangement, with the flowers placed between, and always lower than the leaves. ligig. 34 shows first a nareissus plant pulled to pieces and its sheath removed, then the method of smoothing the leaves with the fingers to take out the curl, and afterwards the way in which the manipulated leaves are comected in pairs and fours by mean of salivit and the replaced shenth, Iig. 35 shows different artificial combinations of these leaves of which the highly curled are only sparingly introduced into compositions; and in Fig. 36 , is illustr.ted a snull bunch of Jeaves with llowers tied with paper before immersing in water, also a trilincal completed arrangement consisting of combincd groups of leaves and flowers. In Fig. 37 different hinds of twists given to leaves are shown, those in a and v are considered affected and cxagrgerated, and those in $\mathrm{A}$ and $\mathrm{n}$ are preferred. l.eaves which curl are chietly used in hanging compositions. The lengths of the flower stems are made to vary according to the particular season, being shorter in Winter than in Spring ar. rangements. If the blossons droop they are carefully straightened and held upright by small 
wooden spikes. A simple five-lined composition with narcissus is shown in Plate 1.XIV.t

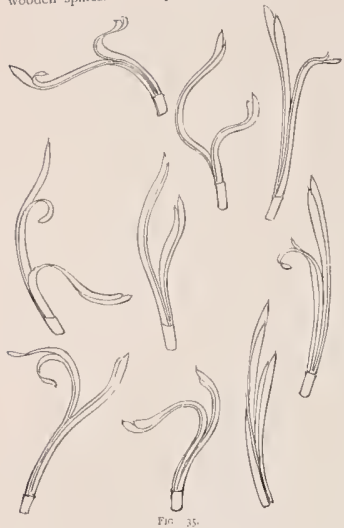
a double arrangenent of willow an marcissus is illustrated in Plate L.I..., an in Plate Xilith... may be seen at trigh composition in a ligh bamboo vase, I which the marcissus occupies the centr: position, with plun branclics ahove an chrysanthenums below.

\section{ARRANGENIENT OF CHERRY BLOSSUMS.}

The chcrry blossom, being r gardecl as the king of indigenous flowe has precedence of all others for floral rangements. It is considered preferable to use branclies of cherry blossom alone, and they are but rarely combined with other flowers.

Somctimes, however, combinations with pine branches, with rape blossons. (Bmassica chinensis), and with irises, are to be secn, though they are hardly considered orthodox designs.

It is said that, in making arrangements with cherry blassoms, the scissors must not be used, Though this rule is not strictly followed, the branches slould be trimmed and bent as litte as possible, and the composition must partake more of a natural than an artificial character. In accordance with the distaste for over-exuberance in floral designts, the cherry of

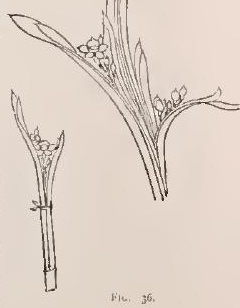


small and single blossom is selected in preference to the richer specim nes of double. blossom.

A composition made with cherry fowers shoukt huve a somewhat sparse arrange. ment of buds at the tup of the central line, half-open blos. soms in the middle, and a few in full bloom below. Several failen petals should be placed in the water of the vase, and any blossons which have dropped may be keft remaining on the flower stand. From the alovev rules it will be observed that the prevailing idea is to represent the cherry-trce as nearly as possible in its naturil state in which the lower branches are the first to burt into blom. The scenery of picturesque spots famous for the wilk cherry-tree, supplies the motive for certain compositions. In Fig. 13. page 68, is shown a simple bunch of blossonis arranged in a small fower-basket placed on a miniature raft, intencled to suggest the flower-lacten boats which font down the sivers at Yoshino and Arashi. yama during the cherry season. In Plate LVI, at "I, is illustrated a composition made with sprays of the weeping cherry placed in a hooking flower-baskct.

\section{ARRANGEMENT OF PEACII BLOSSONS.}

The peach biosson can hardly be said to receive attention in Japan in proportion to its great beatuty

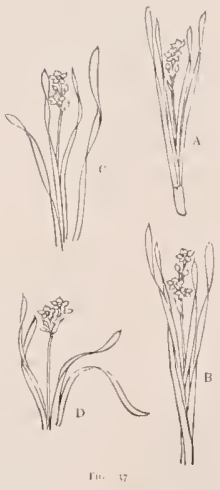
and richness. The peach trees in flower form an attractive feature of the Spring landscape. but their gathered branches, though often employed in floril designs, arc somewhat too exuberant for the taste of the flower artist. The peach is used chiefly in flower compositions during the girls' festival of the third month. The cuttings are then considerably thinnect ont. most of the full-blown flowers heing removed, and only a small number of half-open blossoms and buds left distributed in masse's amongst the folinge. The flower of double blossom is never enplojed, as it is said to have poisonous propertics.

In thate L.XI: it $\mathrm{s}$, is shown an arrangenent of peach blussoms in a standing flower-basket, together with the Rosa indira, and in the same illustration at $n$, the peach is arrangred alone in a hooking basket. 


\section{ARRANGEMENT OF CAMELLIAS.}

The red and white camellia, both single, and doublc, are much used for Spring arrangements, though generally in combination with otlw'r trees or flowers. Their employment in conjunction with the willow has been mentioned in discussing willow compositions. There is a prejudice against the camellia on account of a peculiarity of its blossom, which does not fall to pieces petal by petal like other flowers, but drops off bodily, sugt gestive, it is said, of a head struck off with the sword. On the other hand, it is held in considerable estimation as a tree indigenous to Japan which has been mucla used for various purposes from ancient tines. It is also highly esteemed as an evergreen in floral designs the disposition of the glossy oval leaves receives the greatest attention : two Jeaves at least must be appended to each bud or blossom introduced into the design.

Sonetimes these two leaves point upwards, extending above the flowers; somutimes they are bent over more horizontally, so that the whole of the blossom appeart above them. The white cansellia, if in season, is much used at Coming-of-age celebrations. The favourite hind of composition is one of five blossoms and fifteen leaves, tach flower or bud being surrounted by three curling leaves.

Examples of designs in which the canellia is introduced may be seen in Plate Lil,n, where it is combined with the willow in a suspended bronze vase of crescent shape in Plate XXV.1, it is connccted with the peach and narcissus. In Plate LI, a particular kind called the Camellia sasanqua is shown, arranged with the willow, Bamboo vases are specially suited for camellias, those of the Lion's-month shape being selected by preference.

\section{ARRANGEMENT OF: CONVOI.VUL.L.}

The limp and delicate stems of creeping plants like the convolvulus and morning glory: are difficule to arrange according to the lineal rules of the IFlower Art. Thin bamboo rods, withered stumps, or twigs, are used as a basis in forming a composition, and the design consists in a judicious balancing of leaves, flowers, and buds, in which can be detected the radical clements of Principal, Secoudary, and Tertiury. For the lower portion of an arrangement, flower buds are preferred to open blossons. Rikiu is said to have originated a composition consisting simply of one flower and one leaf of the con- 


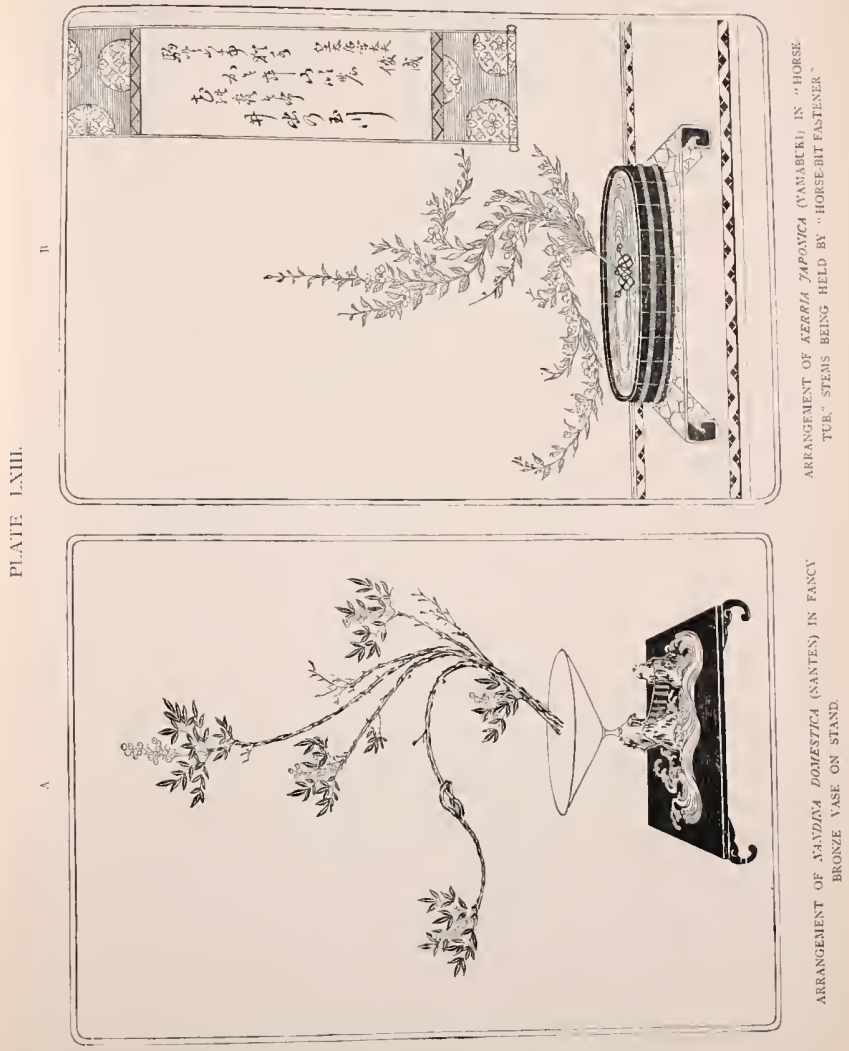





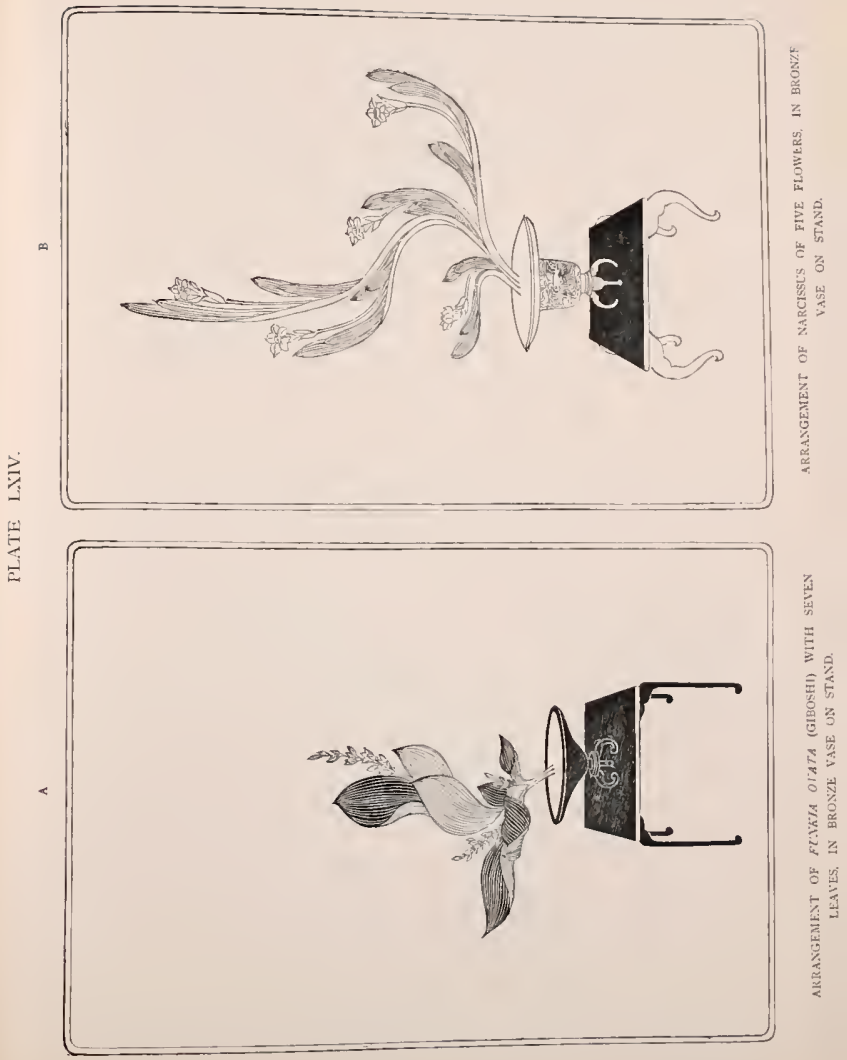





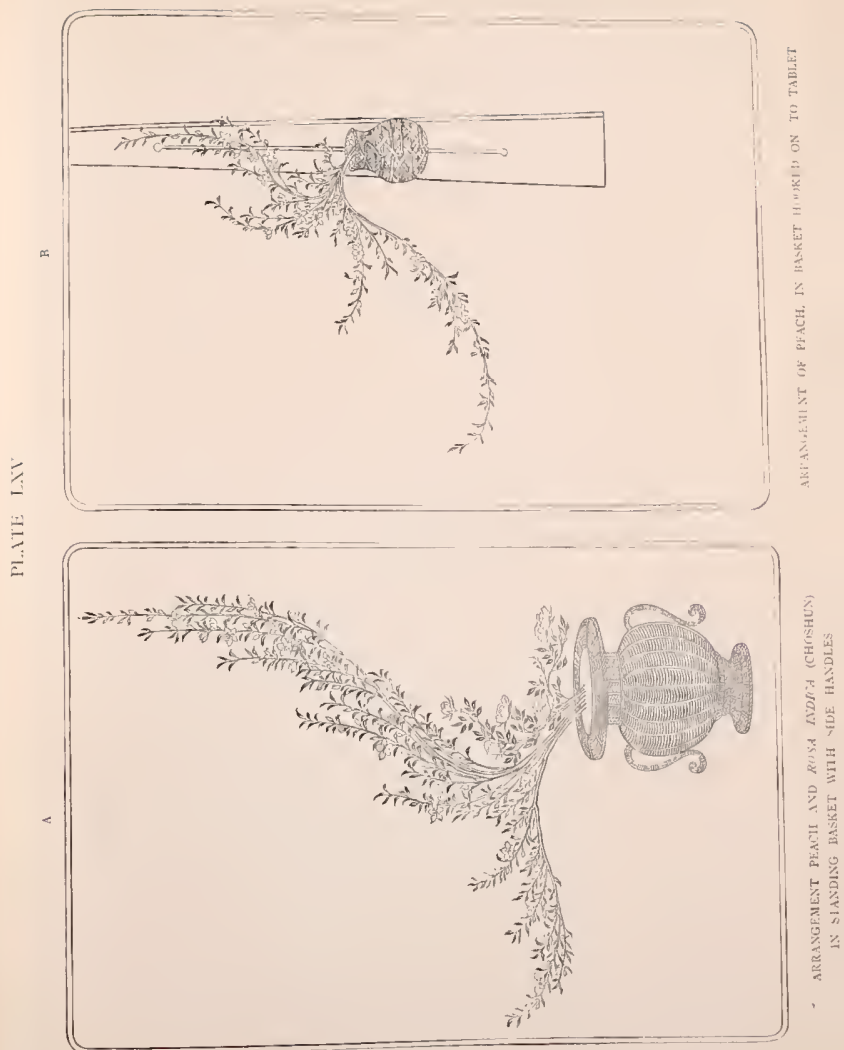



volvulus, which afterwards became a favourite decoration for Tra-mams Upon being asked why he adopted so modest a design, he replied that, as it was impossiblc to rival nature in her grouping, artificial arrangements should be as unassuming as possible; teven a single flower with one leaf being sufficient, he maintained, to call for admiration. One is temptcd to believe that the difficulty of arranging creeping plants according to the accepted standards of lineal balance, may have had more to do with such a departure than the experienced master was ready to admit. The founcler of the Enshiu School invented a nethod of employing the convolvulus in a Hower basket, by winding the creeper round the long oval handle of the receptacle. Such a composition is illustrated in Fig. 6. page 5 . A convolvulus of three blossons, placed in a standing vase on a high table, is shown in Plate L.Mi.A.

\section{ARRANGEMENT OF LESPEDEZA FLOWERS.}

The lespedezat is the principal of the Seven Plants of Antumn, and is much used for foral designs at this season. On account of its numerous small oval liaves and tiny flowers, a full and crowded arrangenzent of sprays is generally resorted to. When placed in standing vases a trilineal composition is followed, each line consisting of a group of four or five stems richly loaded with leaves and blossoms. The lower or Tertiary line nuy be composed of a number of short stems only sparsely supplied with leaves and flower buds, ancl curved in such a manner as to suggest the profile of a wild boar's head. The wild boar is supposed to sleep bencath the Iespedexa branches, and is associated in ant with this Autumn plant. There is an early specimen called the Summer lespedeza, and this should be arranged in a thinner and more open manner than the Autumn plant, in order to preserve its special character of growth, which is less profuse than that of the later season. In hooked or suspended receptacles, the lespedez is employed with a lons Sircanter, the other brinches being kept short and disposed in a simple and quict manner, Such an arrangement, placed in a crescent-shajed vase, is illustrated in Plate LIII.n, The lespedeza is often arranged in suspended bronze boats. It is aiso sometimes used with the morning glory, the Enlaria japonsa, I'aleriana sillosa, Ialcriana oficistalis, Phorriti lhubcrgiam, and the carnation: making together the combination called the Seven Plants of Autumn. 


\section{ARRANGEMENT OF THE RHODE.T Y.HPON/C.A.}

This water-plant is valued for its beattiful large leaves, which are arranged with the greatest care and precision, each inpportant one receiving a special name according to its position or function in the design, as follows :-

The Central-teaf, which occupies the place of the Primcipat, or the nitdle point of the arrangement. The Spring-kcaf, a young and curled leaf enveloping the central stem. The Antumn-leaf, placed in the background of the design, and having a faded or withered tip. The Dere-suppoting-liaf, employed in front of the Princifal. in Spring and Stummer arrangements, and having a curl upwards. The Dezu-spilling-lcaf, used behind the Irimripal in Autunn and W'inter designs, and lnving a curl downtwards. The Szeallenis-month-leatits consisting of two young leaves lapped together so as to present a double tip, ansl placed between the larger leaves of the composition. The Frost-protectiug. leaf, which bends over some distance above the berries of the rhoden, as if to shcitrr them from snow or frost. The Wind-pivteding-feaf. which also curves over the berries sonewhat lower in position. as if to screen them from wind. The ferry-proteding-lanes, being two or three leaves disposed below and around the berries and from between which they appear to spring : in some arrangements these consist of withered leaves. It is considered imperative that, in floral designs made with this plant, the leaves shon!d exhibit a proper balance of frome and kack surfaces. The colour and gloss, to which great attention is given, are much improved by sprinkling the leaves with sake, and immersing their
stems for several hours in the same liquid.

In disposing the leaves in a composition they are piled in a step-like manner in pairs and threes. In this way, for a seven-leaved design, first a pair of Jeaves are placed, then another pair, and lastly three together; the berries are always introduced low
down, screened and partly hidden by the foliage. follow:- - Arrangements of six different styles are recognized for the Rhader japonica, as

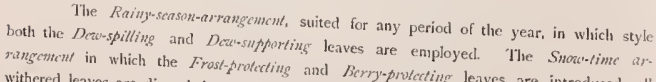
withered leaves are discarded, and the berries are 
tion. The Spring armangonest, suited only for the early months of the year, in which many young leaves are used. The Summer arrangenest, distingruishod by the introduction of a number of large leaves, with the autdition of one withered leaf in front. The Antums-arrangencut, in whicl several withered loaves are placed and no young lenves allowed. The "inter-arrangemen, consisting of a majority of withered leaves with the atidition of the Szrallowis-month-kcaics.

\section{ARRANGEMENT OE LEAF ORCHID,}

The Chinese orchid, called Bumm, is one of the principal stubjects for leaf arrangement in Japanese floral design, and its treatment serves as a molel for most compositions mate with lange-leaved plants. The flowers, being small and insignificant. are often omitted. but, when employed, they are attached by means of thin spikes of bamboo to raise them slightly in position. The bitim requires very careful treatment in order to preserve its freshness, It must be cut in the early morning or after sundown, and its Jeaves are then curled up, tiecl with string, and immersed in water for some hours before use. In very hot weather it is customary to suspend the cuttings for half a day in a deep well. To give a grood colour and gloss to the leaves, sake is forced up their stems before immersing in water.

This plant is generally arranged in a water-basin, sand-basin, tub, or other widemouthed receptacle, with the addition of ornamental stones. In its natural state the leaf. orchid has alway's one long oval leaf growing centrally and higher than the others, and in floral compositions this is used as the Primcifal, and is called the Eud-leaf. The bottom leaf of a series is small, with its point arching over: it corresponds to the Tertiary in floral arrangements, and is called the Finishing-lanf. Another special leaf introduced into certain compositions is one curled up spirally, and callal the Spitcr's-lcaf, leing copied from leaves whicls are curled by the spinning of insects. This form is artificially produced by heating. A ragged leaf called the Decayct-leaf, made ly tearing and scmping, is occasionally added. All the leaves of a composition inchiding the above, are arranged in positions corresponding with the raclical lines of a floral design, each leaf counting separately in the combination. Thus, in a threc-leavect composition, the Extd-linf will be used as Princigal, the Finishing-leaf. Decayed-leaf or Spider's-leaf as Tertiary, and an intermediary leaf will occupy the position of Serontary. In the same manner, for a larger composition of thirteen leaves, the Finishing-leaf is placed as Principal at the top and 
centre of the design: to the right and below are arranged four leaves called respectively

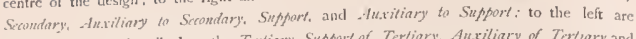
Axcd four others, deseribed as the Tertiary, Support of Tertiary. Anxiliary of Tertary and a Jecovedteaf: and whong the centre, round the stem of the Priciful, are placed four nore, naned in their order from above. Suppont of Irrincipal. Side-picce, Trunk-puter: and Anviliary to Trunkepice.

All the stems of such a composition are closely united in a single line at the base for a distance of several inclies above the surface of the watcr, and the leaves in most cases overlap onc another considerably, only a few of the important ones revealing as much as two thirds of their length. The distribution and balance of leaf surfaces receive considerable attention, so much so, that each leaf, in addition to its other names, is distingrilished by the terns Fnm-smyfuri-lenf or Buck-surfac-leaf. All leaves are curled or twisted in some way, to show a portion of both sides, but rarely in equal degrees, sis that a Froml-surfaci-lenf woukl reveal only a point or edge of its back surface. In Plate: L.t. this balance of surfaces is clearly indicated by shading. It is said that in . composition consisting of five leaves, three, inclusling the End-lcuf and Finishingolenf. shoukl be Froul-surfore leaves, but it appears that no strict rules are followed in this respect, a judicious balance and pleasing variety being alone sought.

Plate XLIV, is instructive as showing defective and corrected arangenents of the Leaf orchid side by side, in which not only the lines but the surfaces of the leaves are altered. An elaborate tlesign, with the same plant in a hexagonal bronze vase, is illustrated in Plate I. . . .

The Leaf-orchicl is occasionally used in combination with other trees or plants, sonctimes as the auxiliary; and sometimes as the principal nember of such double compositions. It is to be seen occupying a subsidiary position combined with the linding domestica. Firsythio snspensa, and large clorjsanthemum, and with the small chrysanthemum and Papraver rharas it holds the principal position.

\section{ARRANGEMENT OF MAPLE BRANCHES.}

The maple, rext to the pine, is the most important flowerless tree used in Japanese compositions. Of this tree, there are two kinds,-the Spring maple, which is red when the young leaves open, and the Autumn maple, which is green in Sunmer, and 
turns crimson later on in the year. Floral artists follow several fancy styles of arrangement with maple branches. which are as follows:-

The Sunvisearrangement, in which the leaves of the Primifal branch slould display their front surfaces. The Sunst-arrangenknt, in which the leaves of the Principit branch should have their under-sides turned to the spectator The (loud)-arcalher-style. in which leaves shothl be curled and sprinkled with spray. The idea of this hast style of composition is taken from the appearance of the wild Naples of Orrararyma near Kinto, the leaves of which are often eurled by frost. The Timen-arrengsment, in which green leaves are used in the upper, and red leaves in the lower part of the composition. Tlue name refers to a spot called Tsuten, famous for its maple trees, the leaves of which redelen from below: The Tatsuta-arrangement, so called from a place called Tatsutu, where fine maple trees line the banks of the stream. In disposing the Rrincipal mass in this composi. tion. several of the larger leaves should be removed and placed Hoating in the water of the flower vase, to suggest the leaves which fall off into the river,

A combination of maple branches with chrysanthemuns is shown in thate XXV.1. The maple is often used in water-basins, and sonnetines in combination with the iris or other water-plants.

\section{MIISCELLANEOUS.}

The above description of special arraggenents with certain tlowers includes those unost often introduced into Japanese floral compositions. Nearly every tree and plant, however. the blossons or foliage of which possess any beauty or attraction. may be seen occasionally introduced into clesigns, citler singly or in combination. The manner of treatment and rombination is based upon the principles already expounded as to characteristics of growth, locality, sex, and season, controlled in many cases by traditimal fancies.

The examples of compositions with leaf-orchicls, fhoder fupritia, lotuses, and Ninphar japonicum, may be taken as models for arranging most phants having latge oval leaves; the designs of irises and marcissus may be followed in employing plants having long, blade-like leaves; the arrangenents of clematis and wistaria illustrate the manner of treating trees and plants of the creeper varicty; the compositions with plum, cherry, peach, 
and willow branches serve as examples for the disposition of other straight-branched and blossom-clad trees: and the treatment of chrysanthemums and peonics can be tuken as a gruide for arranging most plants having large ponderous blossoms.

It only remains to allude to a few exanples among the accompaying illustrations which have received no special notice in other parts of the work.

Plate LXV.N. shows an cxample of the Rosir indica, (Choshun), arranged as at ausiliary in combination with branches of peach blossons in a large flower basket.

Plate XLIH.n, illustrates the Hex sicboldi, (Ume-madoke), in a triple arrangement, placed in a high bamboo vase.

Plate LII,A, exhibits the Tcoma grasdijlora, (Nozenkazura), in a suspended crescentshaped vessel, balanced by a separate design of Calcudula officinalis, (Kinsenkwa), in a standing vessel. ing vase.

Plate XLVIII.s, shows the cabbage-plant. (Ha-botan), arranged in a globular stand-

Plate L.XI.n. illustrates the Dianthus superbus. (Nadeshiko), placed in a bronze vase.

Hate LXIII.r. represents the Funkir o:ata, (Giboshi), a large-leaved water-plant, in an arrangement of seven leaves.

Other plants occur in plates which are intended nuinly to illustrate difierent receptades for lfowers. Among these may he mentioned:- the purple magnolin, arranged

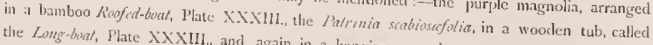
the Long-bat, Plate XXXIIl, and again in a hanging vessel on a Florer-horse, in Plate attached to a Flow shown combined with small clurysanthemums, in a crescent-slraped vessel unbrella, in Fig. If page in Plate LVII. 


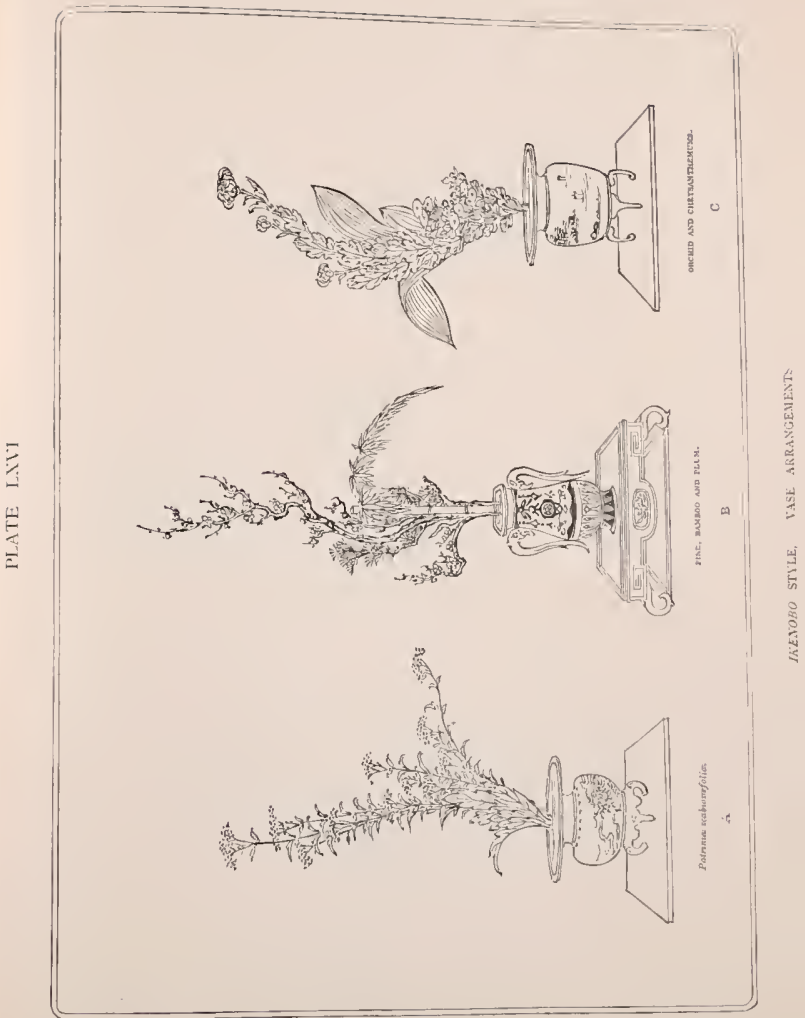





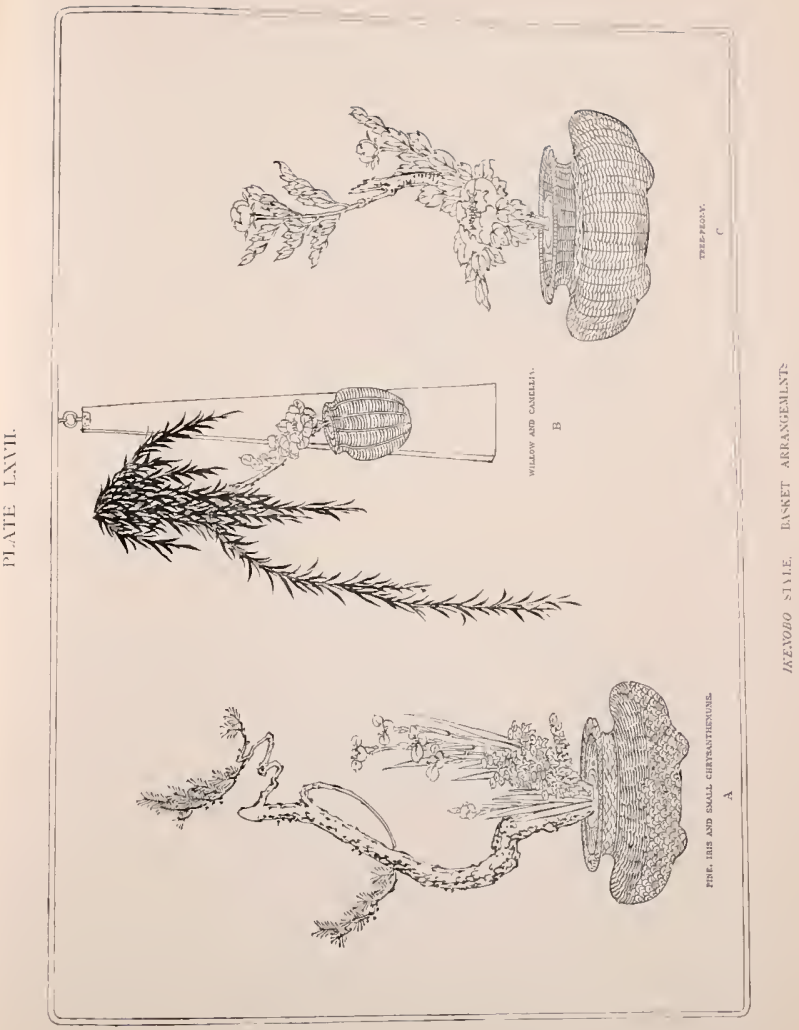





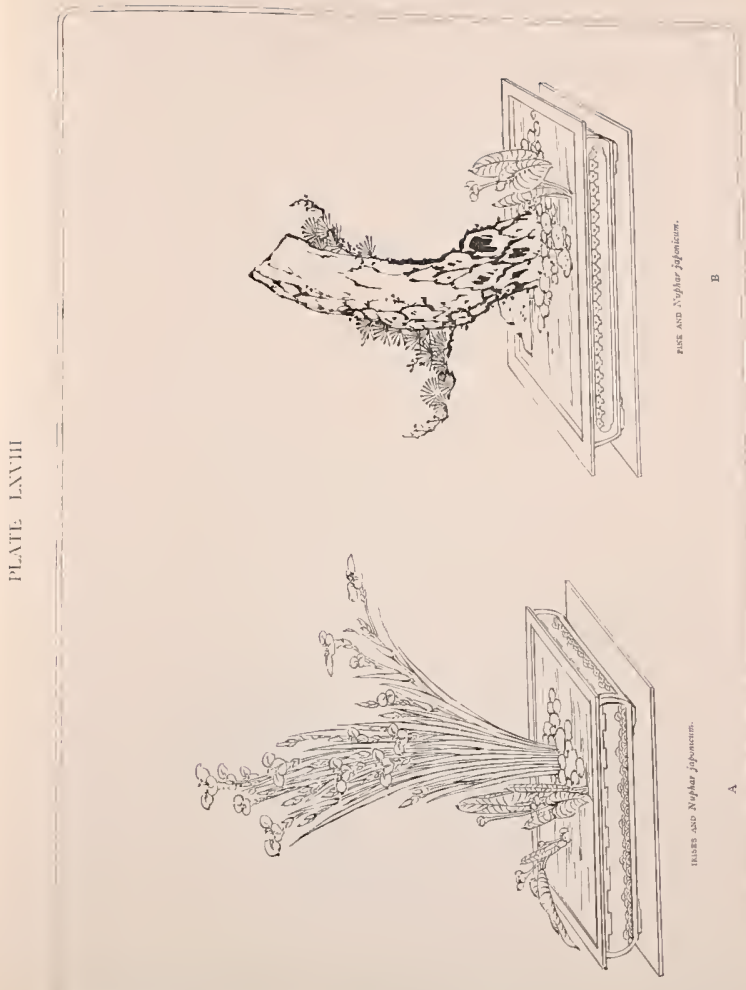

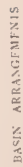

$\frac{2}{5}$
$\frac{5}{5}$
$\frac{8}{5}$ 


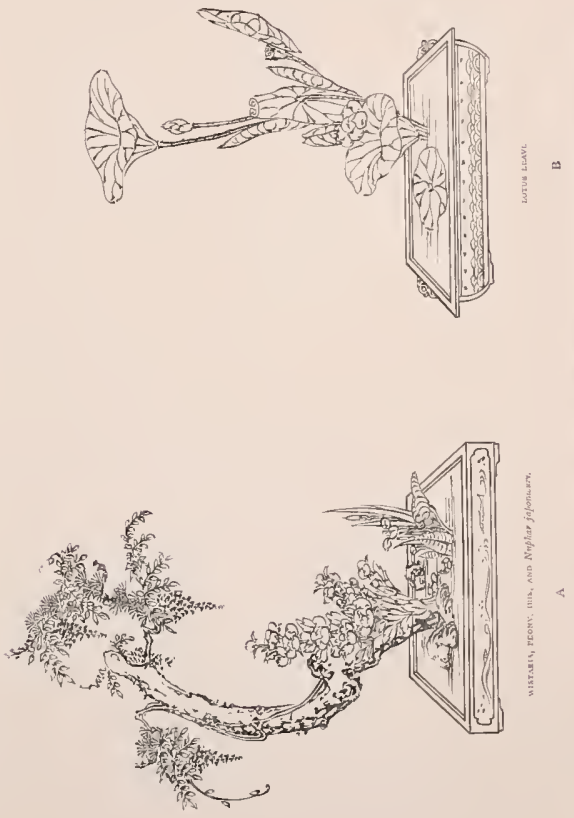



\title{
APPENDIX.
}

\author{
RIKKWA STYLE.
}

$\frac{1}{23}$

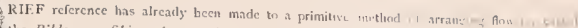

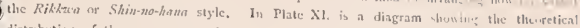
distribution of the seven governing lines in a Rrkput composition. Shap, here meaning 1. core or centre, refers to the central and vertical line or mass, Sishin in floc mame geisen tra a smaller mass just below the S/rim, and in the same line with it, Soys, meaning adjunch, is the principal lateral member on the left: Uke, meaning dependent, is the most impurtaut of the lateral members to the right, Above the l'he is the Hatwsh, meaning distanes, and below it in

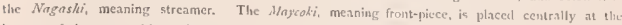
bottom of the composition. Another line or mass called the Do-whrn, meanung trunh-piece, is often added to these on the left, thus making seven lines in addition ter the slin, rut cuntral member Stili an additional member called the Hikaje, or support, is occasinually intenduced, lnut it is not considered desirable in the most correct compositions.

The Surx is the principal line of a floral composition. Theorerically it shoukl be central and perfectly vertical, but in the less elaborate sty.les it is often much bent and diverted. It is said to hold the same relation to the six or scven other lines of a Rikheed arrangement that a lord dues to his vassals, The former, therefore, should exhibit stateliness and repose, whilst the latter express force and movemest. The auxiliary members of a composition are meessary to itv harmony and completencss, but like the various instrumeatalists accompanying a graceful dancer. they are administrative cljiefly to the central object. By such analogies as thest, do uriters explain the relative values of the different parts of a Rikisea design.

Matcrial of a thick and heavy nature, either in stem or foliagc, should be avoiled for the Shin. Its characteristics should be straightness, height, and lightness, $\Lambda$ brancls of young jine is frequently chosen, on account of its erect claracter and pyramidal termination, whicli mahes a suitable apex to the composition. The pine, moreover, being regarded as the king of everpreene, has a symbolical value which renders it particularly adapted in ceremonial decorations. Anciently: this tree alone was selected for the central member or Shia. Altewards, other material hecame nccasionally substituted, preference being alway's given, lvowever, to trees or plants of an erect aut attcruated growth. The bamboo, willow, nandina, fir, oak (Querchs dentatir), plum, juniper, persimmon, and eularin, atre all sometimes employed. The material of the Shiu to some extent controls the selection of miterial for the other parts of the design. The wistaria. willow, plum. uandina, and certain other growths, must, if used in the Shiu, be repeated or echoul in some of the secondary lines. Mostly different species of the same genus are preferred for such repctitions. 
If, for example, the mountain wallow (Safir patifaria) forms the Shim, the river willow (Sali, furfurcal is placed at some nther point is the tesign,

When the wistaria is used, an old stump of some bardy tree must be placed in conjunction with it. In all cases where thick branches of scmi-decayed or lichen-covered trees are employt for the Shim, it is much reducel in height, otherwise it has a top-heavy appearance.

A perfect verticality of the shin is only maintained in the most correct designs. In the. less formal arrangements this member is more or lese bent over to nne side. It is, however, onls in the roughest styles that it is allowed to project beyond the edge of the flower vessel: and erum in free arrangements censiclerable care is devoted to the powerful and vigorous posing of this central feature, which nast never appear weak or unstable. No lateral curvature must necur untul it reaches a point a feu inches above the surface of the water from which it springs. Fhe Shru is the first member of a floral group to be fixed. and its effect must be carefully studied before proceeding to attach nther branches.

The SEISItN is placed centrally in the composition, just belou the Slin from whence it derives its name of atexiliary or small Slum. It is sometines arranged so as to hirle a porlion uf the stem of the Shin, anil is therefore called the Shiw-concealer. In eases when the Shin is bent. the Scishin maintains its verticality and marks the eentral line of the composition. Theorctically the top of this member should be about half way between the tottom and the apex of the composition, though this varies in practice according to the kind of material employed. Some plant, grass, m merely to hide the growth, not ton full or leafy at the top, is generally selected, as its function is he used for the Seishiu, for thene Slim. With the diverted Shin, however, a leafier material may correspondence in growth must be secupies the central gap left in the composition. A certain young pine be selected for the Shim, the $S_{c f s}$ bis shenid these two members Should a branch of or if a plant be chosen for the former, the fatter mist be a phant of somewhat some other kind of pine

The SOYE is the higlaest member placed laterally in a composition. Anciently it was called the Tsuyn-nke, meaning Dewhereizer. on accomt of its form arching at the side of the to the portion of the Shiu above the jumetion. It should be bent), and should be about cqual in length is usual in the most correct designs, the latter is should contrast in claracter with the Shin. If, as of soft and pliant form; but if, on the otber is straight and powerful in line, the Soje should be selected for the Soys. The same kind of ext band, the Shin is much bent, stiffer material must be that of the one is bare and opcn, that of the nost is observed in the nature of the folinge:- when

Following the above prineiples, the Shin having been first placed in position, a branch contrasting with it in character is chosen for the Sope, and this is adjusted with as little Jabour
as possible. Much manipulation is apt to produce a weak and artificial effect. The ibuki 


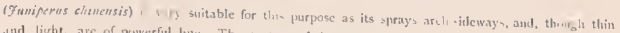
and light, are of powerful hate The conteitir of the soye bemg af avere or less arelied character, it follow's that when plants, such as the chrysenticmem, having heavy blossome are used, the flowers tend to hang face downwards: thas is considered most objesturnable, and several devices are cmployed to keeja the blossoms turned upwards. In spenking of riglit and loft in a Rekkien cramposition, the terms are applied is if the vase of flowers were a pirson facing the spectator, and ate therefore the reverse of the spectator's own right and left. The Soye is generally pluecd on what is called the right side of the S/hn, appearing nu the left side of the iflustration.

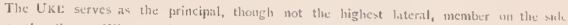

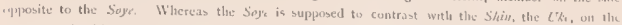
contrary. should accurd in character with it. It follows, therefore, that the lke contrasts with the Soge. When the Shum is large and heavy, the like should also bave similar nature, A suff. straight, and poweriull Soye calis for a bent and pliant Uk'; also, if the limmer be lengthy, the latter should be somparatively short. The porsition of the CKie is somewhat louer than the soye, and the two must never be sxactly opposite with a bent Shim, the ('ke should epring from a print half May betwecn the top of the hase and the divergence of the Shin, and it slmula curve in a direction contrary th the eurve of the She Some growth contristing with the material if the Sige must be used for this member. If the latter be a sprig of willow, a branch of fdram may be aclected for the Uke. In flowers arranged for relighots purposes, the Lke brancli has a my sterious neanitis anul

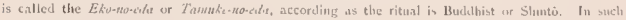
cases, this branch is made to point in the derestion of the relic or image before which the for.al design is placed.

The Mikustri is the fourth line of the compasition. The mime is dufficult to trunslate intelligibly by any simgle woord. The meauing is "seen beyonu," and thi term is frequently applicd to objects in a landscape which suggest distance, such as trees viewed buyond a hill of at

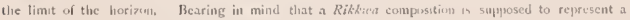
landscape, the Mrikeshi branch mity be tathen as some form in the far-off probsect. On this account it in necessary to avoid using for this member branches of youms trocs, of tall plants, is

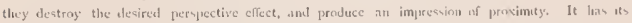

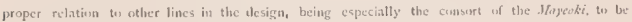
described laxter. with which it should agree in character-soft of rigit, straight or csonked, lught or heavy. On the other hand, it stould contrast with the Scrshen, near whel it in placent. The Mhkosh gencrally -prings from a point below the origin of the Sishin, is of considerable length, and, thougls arraiged more or less centrally, las a slight lateral bend in the direction of the Uhi: It terminates smnetimes above and sometimes below the level of the Scishon Decasinally it is called the guardian to the back of the Shin, because it is the most important of eletse branches which spring form the back of the composition.

The NAGAsul is the lowest laterat brangh in a compasitual. It spreath to cunc ciule in a sweeping form, arching, dipping, and again rising a liule at the extremity. Tlie wnd must 
rever droop, and it should come somcwhat forward in the composition. The Nagraslit springs from it point about three inches above the mouth of the vasc, being about lalf way betwcen the lattur and the origin of the $t \%$ re, and must always have a slight stilt before its bowed form commences, so as to produce a powerful line expressive of vigorous growth. It ought to be the lowest branch of the compusition, and the longest of the lower members. lieing placed on the same side as the CKe, it is important that the two should not terminate cxactly one vertically above the other. The two slould also differ in kind of material. In rare cases the Nugrashi occupies the oppositc side together with the Soye, and then it is made to contrast with the latter, instead of with the Lkc. Any growelh adajted to the long sweeping forn required may be used for the Norgashi. In arrangements of pine brancle's this particular line is called the "incense-burner branch," or exe "smoke receiver," hames lawing reference th the low ornamental censer which on ceremenial uccasions is placed on one side of the vase of flowers.

The Mayeoks ocespies a front position low down in the design, and has the nature of a bunch rather than that of a line. Formerly, the term included the Dosuduri (now a distince member), making only seven radical parts, inclusive of the Shith. For the Mreyeoki, short bunelyy material with a tendency to specad forward must be selected. It should not, however, be of too leafy a muture. The Rhodia japonica is often employed. The iris, narcissus, magnolia, and cypress, though frequenty used in other parts of a Rikktera arrangement, are not cunsidered suitable for the Afraceki, and the funkia and nupliar are absolutely prohibited because wanting in rigidity. The charaeter of this member, which may be likened to the bow or rosette binding together the buse of the foral group, varies more than any other, according to the rough or finished style of composi. tion. In the most formal style it should be quite central and jut forward without any bend on either side. A lateral bend as it projects forward is allowed in the Gio style, and in the So, or secehiest treatment, it may be quite irregular, even bending away from the spectator. Arranged in a composition having a bent centrai line, the Wingeki must conform in charucter with the Dozukuri, spreading right, left, and forward, and creating in combination with the latter member, a hollow called the grotto. Flowers and leaves of attractive shape and colour are employed to produce a bower-like appearance. Ordisary rules, however, require the Majeoki to contrast with the Disukiuri. if the one is sole or delicate, the other should be rigid or coarse in nature. Much stress is laid

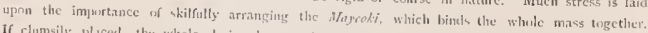
If clumsily. placed, the whole design lones unity and repose. It must on no necaunt hide the mouth of the foner vase. It function is often fulglted hy Jarge leaves, as thosi: of the oak or percimmon tree, which are then subject to all the intricate rules reg-teding the use of Oba (large

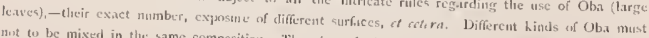
this to be mixed in the same composition. The pinc, rhodea, and pteris, are sometimes called the
tree Alayeali.

The DozUkun, as atated above, was uriginally one with the Maysoki, and only becane distinct in later times. It oecupics a central position, assisting to give fullness to the inass. Generally round and full in form, it is nevertheless adapted to the character of the complete com. 
position, serving to correct os tone down any predominating quality. Thus, should the design appear full and leary, the Dozukuri must be somewhat bare. Flowers alone or mercly butuclics of leaves may be employed. A Dasnkuri consisting of red blossoms is often atled to a floral arrangement of white flowers. It should never sereen or hide the water in the vase.

The H1KAYE is an extra branch seldom introduced in the most correct and formal designs When used, as is sometimes the case in the rougher styles, it is added on the same side as the Soye, and below it. To prevent monotony it must be either longer or shorter than the Soje, and its distanec from the latter depends upon the shape of space or opening which the eye fiyds desirable For the trained artist concerns limself as much with the forms of the spaces of hollows in i composition as with the lincs and masses, just as the skilled caligraphist studies the bollows of an idcograph rather than the touches by which it is construeted. The Hikaye should never be on the same level as the Aigashi, which occupies the opposite side of the floral arrangement. nor shoutd it resemble the latter in shape.

The abowe meinbers may the said figuratively to represent the kelelon, flesh, and smple cluthing of a Rikkwa composition, but, to complete it, otler embellishments are required. Fur these finshing touclies, as well as for certain qualitics of perfection aimed at, there are inmeromterbnical terms, whicls have rather an abstract than a conerete significance, tund need nut all lit cnumcrated here. It has always been the Japanesc art professor's practice to cnvelopre lis teacling: in mystery by the employment of an immense nomenclaturc.

Anciently alt additions of foliage or fowers to compositions, beyond the seven member alreudy described, were called ashirae, or decoration. The Japanese word for gloss or polish is used in a similar sense, having referenee to the attacliment of blossoms or leafy material to spurse and seraggy lines in order to give body and beauty to the whole. Or, such extra material may bo applied in a corrective manner to remove monotony or tone down auy defects in the design. Should the bouquet appear too close and heavy, thin and open growth will be added. Finter work is another favourite expression denoting the filling in of a floral composition with secondary material in such a way as to show up its principal features, just as . th urnament may be disphlayed lefore a sereen, or garden objects against a fence. An inesperienced lanul in liable to werdo this treatment. fucling dissatisficl with simple masses and oprenings in parts of his clesign: but a skilfull designer knows the value of breadth and space, and by their judicions combinatinn will express scencry cfiects. Fetuce-tererk may be applicel to the back or frout of a comarnsition. In the former case, it generally consists of a few plants placed close to the ecutral stem of the Shin and belind the nosmius $i$. Applied to the front, it consists of material alded to the right and left of the Dosmkiri, and in from of the 2 ke and Irikure.

The terms lalley and Grotto are used in reference to certain hollows purpuscly left between the foliage of a design, and intended to be suggestive of landseape effects. The Falley is generally situatcd behind the Dozukuri, and in front of the Seishiu, and is prochecd by a skilfull arrangement of foliage or flowers so as to convey the idea of a shady secre. The Grotio occupies 


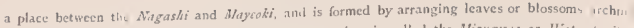

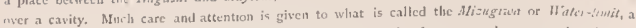
term applicd to the basc of a Rikkter arrangement with speclal reference to the mauser in which it yrings from the basc. This springing should always be firm and united, to convey the dppesrance If vitality and growth, and the bending of different branclses must commence from some littic distance above. This straight connection or stilt which exists below the curvature of the various memburs, is made higher in Summer than in Winter compositions, because in the former it 1 considered refirshing to display as distinctly as possibic the water in the vase.

There modes of arranging the Mfizugriout are practieed. In the first, the unnturl stems if the principal members of the compesition are ieft bare at the base; in the second, a small amount of piant or tree cuttings are added; and in the third treatment, the bottom is made as gay as passible by attaching leaves and fowers. The hast method, though attractive in appearance and thereforc somewhat poptiar, is condemned by the best masters. Some Rikfora armugements affect the cmployment of semi-decayed stumps and branches, in such eases care must be taken that the: Misugitu, or base, is free from decay, otherwise the idea of vitality and growtly is lost. In usinge bamboo stems the distance of the first knot above the wizter is important for similar reasons.

The terms thiz (potier, or spirit) and Utsuri (reflection) are frequently, used conjuntly th describe the forte of line in one floral member alad the reciprocal vigour required in another part of the composition. A potverful S/ain is said to lhave $/ \mathrm{ki}$, and a proper echo or balance of this character in the Uke is called Uisuri. The word Iro in its application to the fornal art has the meaning of natural harmony, When the material employed prescrves throughout its natura. claracter, with no sign of artificial treatmeut, branches of straight fibre being used verticaliy, and branches naturally bent and sweeping disposed luterally, then the composition is said to have lie. Moreover, if there is perfect courcord in character and proportion between the bouguet and the receptacle in which it is placed, the same term is employed to signify the harmony produced.

Riltiven compositions may be broadly divided into two clasets, thense having a straight alud those faving a bent Shin. These two classes have cach their these manners or degrecs of clabosation, named resulctively Shin, Gio, and So. Plates XII., XIII, XIV., illustrate the Sisis, Giv, and So degrees of the straight Shin style. The first and more formal arrangement (Plate XII), is used at marriage cercmonies. It was originally designed to go with the three sacred utensils of a Buddhist altar-the pair of

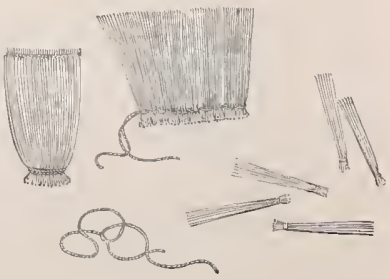


candlesticks shid the incense burner. Fasteners for the extremities at chttings ateanged in the Rikhtor style, generally consist of small bundles of straw or stubble tied together, and placel in the base.

Plates XV., XVI, and XVII. illustrate the three degrees of the bent Shin style. The principal laults to be guarded against in arranging fowers in the Rikkon style are sliown in a fugured diagram of Plate XXI. The errors illustrated are as follow: $-\mathbf{A}, \mathrm{B}$, Two blossoms of the same kind in a line - C. two lateral branches of the same length side by side, D, cross formed by two branclich, F. a branch dropping from the water line ower the vase: F, a branch or sprat coming forward cxactly centrally: G, a large blossoin elose to the uater surface; H, a branch from belind bending rounel to the front, K, placing other material just below a blossom so as to hide its natural leatves: M. tusing the flowers only of a plant which possesses fine leaves; L, a branch dipping into the water: I, a branch tuisting back towards the wall, I. one branch crossing another diagunally

The Ridken Stump arrangements are illustrated in Plates XViI., X1X., and XX. For suth finral compositions a low broal basin or bowl is used, and instead of the ordinary stubble fastening, a wooden frantework is let unto the basin to liola stcady the beay stubs anu branclues used. This framewotk is afterwarcls to a great extent concealed by the sand, pebblew, and water. The Sand-bowl arrangements of flowers, as these are sometimes called, are also controlled by a theory of aeven governing lines. Whereas in the Shin-holhtun or Standing-vase arrangements, the tendency is vertical; in the Sind-bowl arrangements, the tendency is horizontal. The high becomes low, and the narrow broad. In the more frnished style, one thick stump is employed in continuation with other materials, (see Plate XVIII). Here we have a plum stump with branches, combined with pine, camellin, and wircissus,

Plate XIX. illustrates the double stump arrangement which is followed is the rougher style. The idea of sex is applied to such arringements, the stump to the fight being called wak, and that to the left femsic. In this composition various kinde of pine, irises, reeds, and bambon grass are employed.
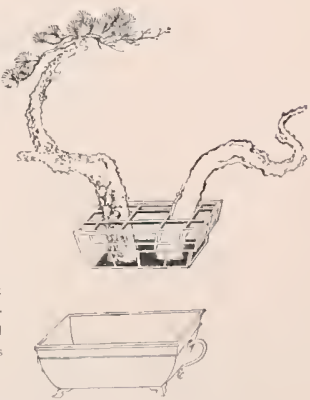

Plate XX illustratus a combined Rikken and Sanct-bowd arrangement, suitablo for Ilacing upon the rinamental shelves of a chamber. The upper arrangement is on the principle of the Shin-uo-hath or erect style, but is somewhat lowered and bioalened out on account of its high position. The lower arrangement is in the rough Sand-bowl style, witl double stump of pine, irises, bamboo, and ferns. 


\section{IKENOBO STYLL.}

The Thonobo style of flower arrangement lixs of late years become somewhat popular among amateurs in Japan. It is the revival of an ancient method, to be traced directly to a modification of the original Rikktiz style, tn which certain rescmblances may be observed. In Plate L.XVI. at B is shown an Jkcbono design with pine, plum, and bamboo, which it is interesting to compare with the Rikhon designs in Plates XI. and XII.

The fictromo school, however. does mit adopt an abundance of different material in one inmpositem is in the Rikewe style, but lumits itself to the combinutuon of two or three granthe. The most strihing elaracteristic of this method is the clase and bunclyy nature of the designs as compared to those of the Eushiu and other schools The lineal character, which is so marked in the lacter, to a great extent disappears, though the outline of the floral arrangements produced contains a suggestinn of the radical lines. The triangular contonr always predominates, and this may be seen very distinctly in Plate LXVT, A, and C., PJate L.XVIII. A, and Plate I.XIX. It

In effect, the arrangements of the Heborto styit appeal to the Eumpean taste as morc matural and less conventional than those of the Eushiu stylc. They are, however, equally subject t.) chiborate rules dot unhle those of the rival schnols. The following illustrations from Ilate LXV1, to Ilate LXIX. give examples of therobo arrangements, in vases, baskets, and sand-bacins 
明明明

治 治 治

二 $三$ 三

$+1-1$

四 : 二

年 年 年

六六六

月月

十 - Ii

日 日

落

發

印印

印 訂

正: 正:

作

行

刷刷

刖再西

出版版

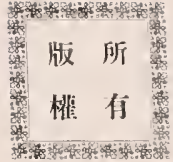

印登

版刷行 英

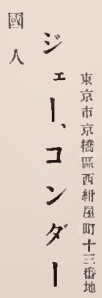

藏

教

都

fi)

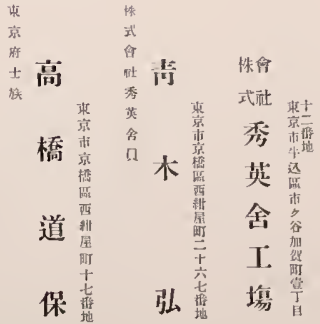







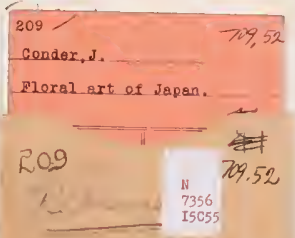

THE OXTARIU COLLECEE OF ART $\rightarrow \geqslant$

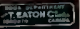




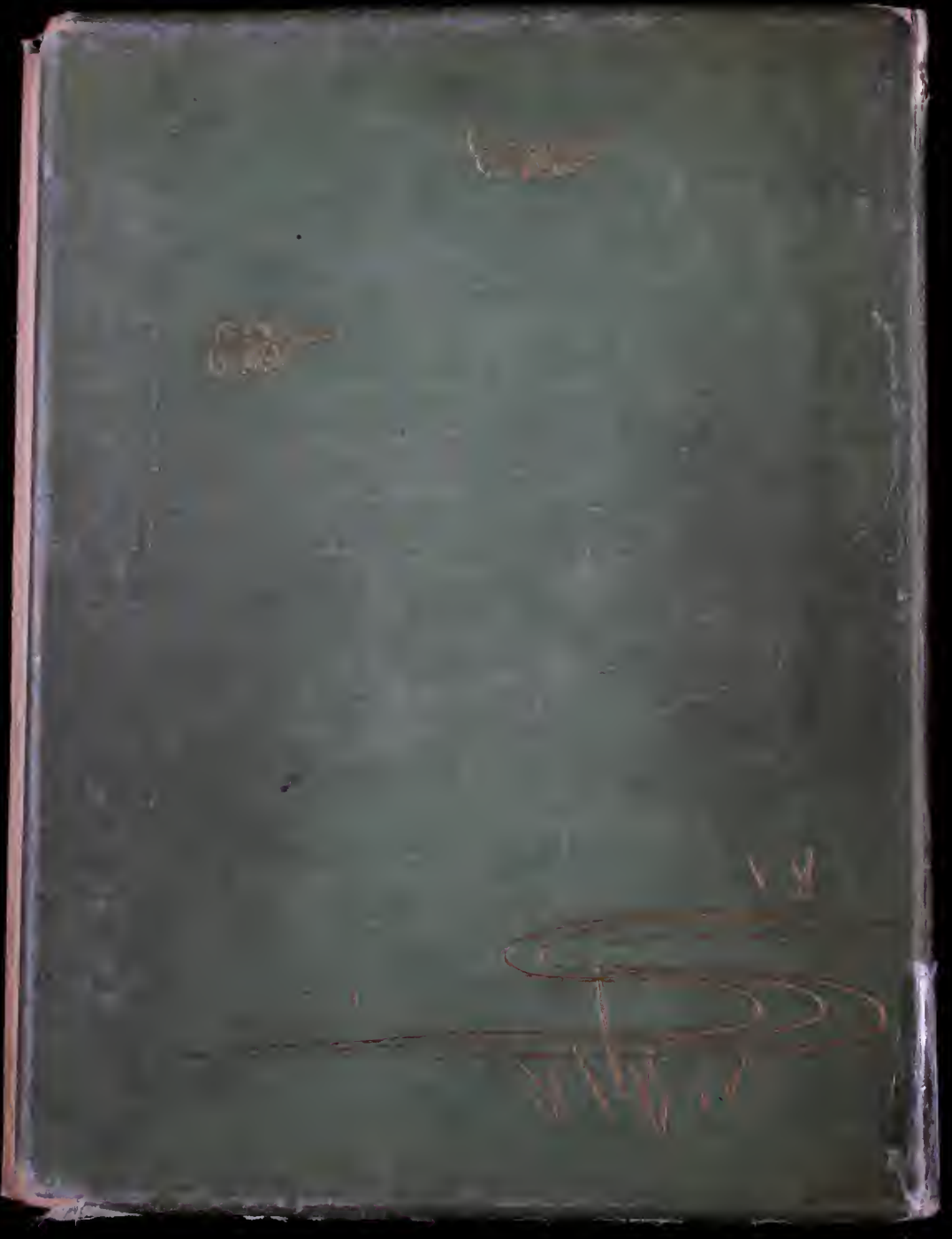

